S E M I N A R I O G I U R I D I C O

D E L L A U N I V E R S I T À D I B O L O G N A CCLXXXVIII

\author{
MARTINA VINCIERI
}

\title{
L'INTEGRAZIONE DELL'OBBLIGO DI SICUREZZA
}

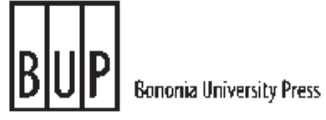


Bononia University Press

Via Ugo Foscolo 7, 40123 Bologna

tel. (+39) 051232882

fax (+39) 051221019

www.buponline.com

e-mail: info@buponline.com

C 2017 Bononia University Press

Tutti i diritti riservati

ISSN 2283-916X

ISBN 978-88-6923-274-9

Impaginazione: Sara Celia

Prima edizione: novembre 2017 
Per la presente monografia la Giunta di Dipartimento ha nominato la seguente Commissione di lettura:

Luigi Balestra (Alma Mater Studiorum - Università di Bologna), per il Settore Scientifico Disciplinare IUS/01 Diritto Privato Andrea Lassandari (Alma Mater Studiorum - Università di Bologna), per il Settore Scientifico Disciplinare IUS/07 Diritto del Lavoro

Sandro Mainardi (Alma Mater Studiorum - Università di Bologna), per il Settore Scientifico Disciplinare IUS/07 Diritto del Lavoro 

Ai miei genitori e

ad Alessandro 



\section{INTRODUZIONE}

Nel d.lgs. n. 81/2008, volto al «riassetto» e alla «riforma» della legislazione vigente in materia di salute e sicurezza nei luoghi di lavoro, accanto al complesso di norme rigide e predefinite, sono contemplate altre fonti niente affatto marginali cui è affidato il ruolo di integrare e completare il quadro normativo.

Hanno, così, assunto notevole rilievo le «buone prassi», «adottate volontariamente» ai sensi dell'art. 2, comma 1, lett. v, d.lgs. n. 81/2008 e volte a migliorare le condizioni di lavoro ed i livelli di tutela stabiliti dal legislatore. Il riferimento è anche agli accordi sindacali, oltre ai codici di condotta ed etici, che orientino i comportamenti dei datori di lavoro (art. 6, comma 8, lett. h, d.lgs. n. 81/2008). Specifici strumenti, su base volontaria, sono, altresì, i diffusi modelli di organizzazione e gestione, il cui utilizzo è stato promosso ed accentuato dal d.lgs. n. 81/2008 all'art. 30.

V'è dunque un concorso di fonti diverse, tutte variamente deputate a garantire la protezione dei prestatori durante il lavoro, fino ad accrescere le garanzie ed elevare gli standard di sicurezza previsti normativamente.

Si delinea, in sostanza, una varietà di direzioni attraverso le quali si possono creare, ad iniziativa del datore di lavoro o in accordo tra le parti, regole indirizzate a definire ed anche innalzare i livelli di prevenzione nell'ambiente di lavoro, completando gli interventi hard di regolazione eteronoma: "fonti" tendenzialmente contraddi- 
stinte dalla introduzione di misure ulteriori rispetto alle previsioni di cui al d.lgs. n. 81/2008 e dirette al perfezionamento dell'obbligo di sicurezza.

Non sono di poco conto le implicazioni giuridiche che questi comportamenti "virtuosi" producono all'interno del rapporto di lavoro; qual è, in particolare, la loro efficacia, soprattutto nel tempo?

Il campo di indagine della ricerca è incentrato, essenzialmente, sulle prassi o «soluzioni organizzative o procedurali», scelte o introdotte dal datore di lavoro, a volte condivise con le rappresentanze sindacali dei lavoratori, nonché sugli strumenti di gestione che possono essere adottati all'interno dell'organizzazione del lavoro, al fine della massima tutela possibile della salute dei lavoratori.

Il percorso seguito è così condotto lungo il "filo rosso" che lega le buone prassi intese quali atti unilaterali del datore di lavoro, le misure migliorative inserite e negoziate all'interno di una intesa contrattuale tra le parti, prevalentemente a livello d'azienda, nonché i modelli organizzativi, frutto di una scelta libera e insieme "necessitata" del datore di lavoro.

La pretesa è, anzitutto, quella di accertare e stabilire quale sia la natura giuridica, la valenza, la portata delle prassi definite volontarie nell'art. 2, comma 1, lett. v, d.lgs. n. 81/2008, all'interno del sistema giuridico italiano al quale tradizionalmente sono estranee; quindi di verificare se tali iniziative siano realmente caratterizzate dalla mera volontarietà $\mathrm{o}$, al contrario, si configurino come prassi certamente buone, ma doverose.

In proposito, la riflessione concerne sia l'eventualità che l'opzione datoriale resti circoscritta all'interno del perimetro aziendale, sia che ne esca, assumendo una valenza pubblica per il tramite dell'apposito meccanismo della validazione ${ }^{1}$.

Viene fin da subito in rilievo il problema dell'esatto raccordo con l'art. 2087 c.c., da sempre al centro delle riflessioni degli interpreti, nonché delle decisioni assunte nel giudizio civile e penale. $\mathrm{Ci}$ si deve, infatti, interrogare su come conciliare l'adozione volontaria

1 Cfr. l'art. 6, comma 8, lett. d, d.lgs. n. 81/2008, ai sensi del quale è attribuita alla Commissione consultiva permanente la validazione delle buone prassi in materia di salute e sicurezza sul lavoro. 
delle buone prassi con l'adempimento dell'obbligo di sicurezza. I dubbi interpretativi concernono cioè la possibilità che le richiamate prassi contribuiscano all'adempimento dell'obbligo, quali «misure necessarie», ovvero addirittura vadano oltre lo stesso disposto dell'art. 2087 c.c.

Nel variegato e complesso panorama delle possibili soluzioni tecniche, organizzative o procedurali finalizzate alla tutela della salute dei lavoratori, la risposta non può, però, essere univoca, bensì articolata.

Assumono importanza la qualificazione giuridica delle menzionate regole nonché le conseguenze della loro applicazione sui profili di responsabilità datoriale. L'indagine si giustifica proprio in quanto nella specifica materia della tutela della sicurezza nei luoghi di lavoro, l'ordinamento interno è tradizionalmente connotato da norme eteronome e, in particolare, dall'art. 2087 c.c.

V'è, allora, da chiedersi se tali prassi migliorative abbiano modificato il panorama delle regole giuridiche o siano esse stesse regole giuridiche vincolanti: sicché, il "vecchio" sistema e le più moderne buone prassi possono convivere?

In realtà, la modernità, se non «perfezione», dell'art. 2087 c.c., norma chiave per la sua semplicità e completezza «in ragione dell'elasticità del suo contenuto» ${ }^{2}$, si apprezza ancora di più proprio attraverso la riflessione sul ruolo e sull'efficacia degli strumenti richiamati. In effetti, già la norma codicistica, imponendo appunto l'adozione delle «misure necessarie» a tutelare l'integrità fisica e la personalità morale dei prestatori di lavoro, ha in sé, implicitamente e inconsapevolmente, il rinvio a fonti ulteriori.

In merito alla delimitazione dell'esatto contenuto dell'obbligo di sicurezza e dei suoi confini il dibattito, dottrinale e giurisprudenziale, è tuttora incessante ${ }^{3}$. Nell'ambito delle diverse interpretazioni formulate, quella preferibile e, in un certo senso, mediana, pone a carico del datore di lavoro l'obbligo di garantire la massima sicurez-

2 L. Montuschi, Diritto alla salute e organizzazione del lavoro, Milano, Franco Angeli, 1976, p. 76.

3 Si ricordi la nota pronuncia della Corte costituzionale del 25 luglio 1996, n. 312, in RIDL, 1997, II, p. 15, con nota di V. MARINO, La minimizzazione del rischio sui luoghi di lavoro nell'interpretazione della Corte costituzionale. 
za disponibile sul mercato. Pur non dimenticando che la sicurezza è «l'arte del possibile», ciò che si esige pare sempre di più corrispondere alla predisposizione di «tutti i mezzi e gli strumenti disponibili» ed all'impegno «ad una continua ricerca ed ammodernamento» delle misure di prevenzione, in considerazione dei cambiamenti intervenuti nell'organizzazione del lavoro ovvero «dei progressi, spesso accelerati, della tecnica» ${ }^{4}$.

Da questo punto di vista, il rispetto delle migliori prassi ed accorgimenti risulta funzionale all'adeguamento doveroso da parte del datore di lavoro alle modifiche occorse sul piano tecnologico e dell'esperienza maturata, cioè alla garanzia della massima protezione della salute dei lavoratori. Nella consapevolezza che l'osservanza dell'obbligo di sicurezza impone anche l'adozione di «misure innominate» ossia di «comportamenti specifici che, pur non dettati dalla legge o da altra fonte equiparata, siano suggeriti da conoscenze sperimentali e tecniche» ${ }^{5}$.

La legislazione speciale affiancatasi, successivamente, all'art. 2087 c.c. è stata incisivamente arricchita dal d.lgs. n. 81/2008, che sotto diversi profili si distingue da altre normative in materia di prevenzione dei rischi adottate nei diversi Stati membri a ridosso della direttiva quadro $89 / 391$.

Il decreto del 2008 con la esplicita nozione e disciplina delle «buone prassi», altrove per lo più assente, ha innestato in tale contesto una novità di ampio rilievo, che pone delicate questioni giuridiche ed è destinata a far discutere. Occorre, inoltre, ricordare i diversi richiami al progressivo miglioramento delle condizioni di lavoro e all'aggiornamento continuo in base, in particolare, alla tecnica, parametro che permea di sé il d.lgs. n. 81, in ossequio all'art. 2087 c.c.; la stessa definizione di «prevenzione» si riferisce, del resto, al «complesso delle disposizioni o misure necessarie anche secondo la particolarità del lavoro, l'esperienza e la tecnica» (art. 2, comma 1, lett. n, d.lgs. n. 81/2008).

${ }^{4}$ Così L. Montuschi, La sicurezza nei luoghi di lavoro ovvero l'arte del possibile, in $L D, 1995$, p. 415.

5 Recentemente cfr. Cass., sez. lav., 30 giugno 2016, n. 13465, in DeJure; Cass., sez. lav., 5 gennaio 2016, n. 34, in DeJure. 
Tutta la normativa prevenzionistica pare, insomma, costruita sulla non definitività e sul dinamismo, attraverso la dialettica tra obbligo giuridico di protezione e tecnica. Si può ritenere che l'innovazione tecnologica, che incide in modo significativo sull'obbligo di sicurezza, sia più facilmente ed efficacemente perseguibile per mezzo di strumenti ad hoc, confacenti alle esigenze della singola azienda. L'art. 2087 c.c. pare, insomma, aver forgiato il d.lgs. n. 81, nel quale hanno a loro volta trovato collocazione e specifica regolazione strumenti idonei a completare il contenuto della norma codicistica.

È vero che storicamente le «misure necessarie» a tutelare la salute dei prestatori di lavoro cambiano. In ciò si rinviene appunto la dinamicità dell'art. 2087 c.c.

Ci si può, finanche, domandare se le menzionate prassi ed anche i modelli di gestione non siano, in realtà, una metafora del principio di organizzazione, ovvero in un certo senso il paradigma dell'organizzazione stessa, che pervade il d.lgs. n. 81/2008 e nella quale si inseriscono.

Di particolare interesse sono, inoltre, le implicazioni sui profili di responsabilità. Da questo punto di vista è pertinente il confronto con gli Approved Codes of Practice (ACOPs) propri del Regno Unito. Anche gli $A C O P s$, a cui le buone prassi in un certo senso si ispirano, sono intesi quali strumenti deputati a indirizzare la condotta del datore di lavoro; la loro mancata adozione, pur non comportando di per sé conseguenze sanzionatorie, incide tuttavia sull'assolvimento dell'onere probatorio, dunque sull'accertamento della responsabilità. Tale raffronto si rivela particolarmente utile al fine di un'interpretazione in senso critico della stessa nozione legislativa di buone prassi, di cui al d.lgs. n. 81/2008.

Sicché, le soluzioni migliorative, unilaterali o contrattate, e i modelli organizzativi, possono essere considerati misure per adempiere l'obbligo di sicurezza, ossia anelli di una stessa catena; il percorso che conduce all'adempimento dell'obbligo si caratterizza, così, per una sua circolarità.

Ci si propone, dunque, di indagare i suddetti strumenti, valutandone le interferenze o interconnessioni.

Il tema, finora un po' trascurato in dottrina, sta divenendo di centrale importanza nella realtà delle scelte organizzative aziendali; 
la salute, come chiarito, «mal si presta ad essere tutelata attraverso la predeterminazione di regole rigide e $\operatorname{standard} \gg^{6}$.

Quanto precisamente alle buone prassi, oggetto di crescente attenzione anche in altri contesti nazionali, l'Agenzia europea per la sicurezza e la salute sul lavoro ne promuove la diffusione e si premura di pubblicare il positivo rapporto costi-benefici relativo al miglioramento della sicurezza e della salute, mettendo in risalto $i$ «vantaggi economici di una buona strategia» di prevenzione dei rischi sia per le grandi che per le piccole aziende; come chiarito, «le aziende con standard più elevati di salute e sicurezza hanno più successo e sono più sostenibili ${ }^{7}$. Ne consegue che un efficace sistema di prevenzione avvantaggia anche economicamente le imprese, divenendo un elemento fondamentale della gestione aziendale, a maggior ragione se lasciato alla discrezionalità del datore di lavoro e realizzato con riferimento alle specifiche situazioni di rischio. Il messaggio diffuso all'interno dell'Unione europea è, pertanto, che la promozione di misure prevenzionistiche innovative ed ulteriori rispetto a quelle legislativamente imposte, in un certo senso, conviene. L'ambizioso e sfuggente obiettivo da tempo perseguito della "cultura della prevenzione" pare acquistare un significato più concreto.

Das Problem, come è indicato nell'introduzione ad alcuni studi monografici tedeschi, ossia il problema su cui si riflette dal punto di vista giuridico è, allora, reale ed attuale. La regola eteronoma deve, in sostanza, essere supportata dalla autonormazione; del resto, «la legge spesso si ritrae per lasciare campi interi a normazioni di origine diversa», derivandone una «concorrenza delle fonti», su cui è necessario riflettere ${ }^{8}$.

6 B. Caruso, Introduzione, in B. Caruso, M. D’Antona, S. Sciarra (a cura di), Il diritto del lavoro nell'Unione europea. Francia, Germania, Gran Bretagna, Spagna, Napoli, Edizioni Scientifiche Italiane, 1995, vol. I, cap. II, p. 179 ss.

7 Cfr. http://osha.europa.eu/it. Si veda, inoltre, European Agency for Safety and Health at Work. Estimating the cost of accidents and ill-health at work: a review of methodologies, European Risk Observatory, 2014.

8 G. Zagrebelsky, Il diritto mite. Legge, diritti, giustizia, Torino, Einaudi, 1992, p. 47 e p. 168; in proposito cfr. le considerazioni di P. PAscucci, Dopo la legge n. 123 del 2007. Prime osservazioni sul Titolo I del d.lgs. 9 aprile 2008, n. 81 in materia di tutela della salute e della sicurezza nei luoghi di lavoro, Quaderni di Olympus-1, Pesaro, edizioni studio @lfa, 2008, p. 162. 
L'obbligo di sicurezza eteronomo tende a completarsi in una scelta individuale, a garanzia dell'effettività della tutela nell'ambiente di lavoro.

Acquista, come anticipato, importanza anche il profilo della tutela collettiva della salute. Nonostante i modesti risultati finora raggiunti, nella negoziazione collettiva in prevalenza d'azienda sempre più attenzione pare essere riposta, infatti, sull'accrescimento del coinvolgimento dei prestatori di lavoro, proprio al fine di individuare soluzioni condivise e innovative in materia di prevenzione dei rischi. La materia de qua si presta particolarmente ad incentivare forme di "partecipazione", dirette o solo mediate, dei lavoratori alla gestione della sicurezza, in tal modo assurgendo a esempio della realizzazione di possibili modalità partecipative in azienda.

I segnali e le manifestazioni di autoregolamentazione o di accordi condivisi volti ad accrescere ed avvalorare gli aspetti prevenzionistici non possono affatto essere trascurati. Il problema non può, inoltre, prescindere da un'indagine comparata, che necessariamente s'impone, nel rispetto delle «uniformità», nonché delle «profonde dissonanze» tra i diversi contesti nazionali ${ }^{9}$.

L'obiettivo è quello di verificare l'esistenza di modelli simili in altri ordinamenti, accomunati dal recepimento del corpus di regole generali contenute nella direttiva quadro europea 89/391, eppure alquanto divergenti. Vengono in rilievo, appunto, le specificità proprie dei singoli Stati membri, alcuni inclini più di altri a promuovere la formazione di regole in azienda in ordine alla tutela della sicurezza sul lavoro. La lente della comparazione è necessaria al fine della presente ricerca, pur nella consapevolezza che ciascun sistema nazionale è fortemente connotato da complessi apparati storico-istituzionali e tecnico-giuridici. Con precipuo riferimento al ruolo della partecipazione in materia il metodo comparato consente di coglierne esattamente il significato e, nello stesso tempo, di comprendere i limiti della disciplina interna rispetto ad altri contesti ove il coinvolgimento dei lavoratori è maggiormente valorizzato. Gli spunti of-

9 G.F. Mancini, Appunti di Diritto comparato del lavoro, Bologna, Riccardo Pàtron, 1954, p. 6, il quale ha sottolineato «l'importanza teorica e pratica» dello studio del diritto comparato. 
ferti, pur non ambendo ad indicare soluzioni normative perseguibili nell'ordinamento interno, tratteggiano il possibile modello cui tendere.

In ogni caso, al di là delle peculiarità esistenti nelle singole legislazioni prevenzionistiche, si possono fin d'ora scorgere alcune affinità che attengono al significato stesso di prevenzione dei rischi, conformemente ai principi della direttiva quadro 89/391. Così, ad esempio, l'art. 14.2 della Ley de Prevención de Riesgos Laborales (31/1995), oltre a specificare il diritto dei lavoratori ad una protezione «efficace», rimarca l'obbligo incombente sul datore di lavoro di adottare «le misure che siano necessarie per la protezione della sicurezza e salute dei lavoratori»; il richiamato art. 14.2, interpretato in modo ampio e dinamico, riveste, come precisato in dottrina, particolare importanza in considerazione della sua «modernità» ${ }^{10}$. Analoga previsione si rinviene nell'ordinamento tedesco ove l'Arbeitsschutzgesetz, la legge per il miglioramento della sicurezza e della salute dei lavoratori del 7 agosto 1996, nel $\S 3$ pone a carico del datore di lavoro «erforderlichen Maßnahmen des Arbeitsschutzes» nel rispetto delle «circostanze» che influiscono sulla salute e sicurezza dei prestatori di lavoro ${ }^{11}$. Inoltre, lo stesso Code du travail, che racchiude tutta la normativa in materia, sottolinea che il datore di lavoro deve garantire l'adozione delle misure di prevenzione «necessarie» per assicurare la salute fisica e mentale dei prestatori di lavoro, mirando al miglioramento delle situazioni esistenti (art. L 4121-1).

È, però, in particolare nell'ordinamento italiano che si verifica un reale ammodernamento delle fonti nella direzione promossa in più occasioni dal diritto dell'Unione europea e percorsa già da tem-

10 M.T. Igartua Miró, Dos décadas de aplicación de la Ley 31/1995, de Prevención de Riesgos Laborales (LPRL): luces y sombras, in DSL, 2016, n. 1, p. 37 ss.

11 Il $\S 3$ dell'Arbeitsschutzgesetz si riferisce esattamente a «misure necessarie di sicurezza sul lavoro». L'obbligo di garantire la salute nell'ambiente di lavoro è contemplato, inoltre, nel $\S 618$ BGB: cfr. per un commento A. Deutsch, $\S \S 617$ 619. Besondere Pflichten des Dienstberechtigten, in M. Schmoeckel, J. RücKert, R. Zimmermann (hrsg.), Historisch-kritischer Kommentar zum BGB, III, Tübingen, Mohr Siebeck, 2013, p. 1251 ss.; nonché H.-W. Friedrich, $\$ 618$, in H. SchliemanN (hrsg.), Das Arbeitsrecht im BGB. Kommentar, Berlin, Walter de Gruyter, 2002, p. 461 ss. Sull'obbligo di sicurezza in generale si veda già W. Kaskel, H. Dersch, Arbeitsrecht, Berlin-Heidelberg, Springer Verlag, 1957, p. 259 ss. 
po dagli ordinamenti di common law. La legislazione interna, rivista ed aggiornata più recentemente, offre, infatti, l'occasione di riflettere in merito alla previsione di ulteriori procedure e metodi deputati a scongiurare il rischio di infortuni e malattie nei luoghi di lavoro.

Del resto, lo stesso Trattato di Nizza, nell'apportare modifiche al Trattato sull'Unione europea, ha sancito la priorità assunta nell'azione degli Stati membri dalle tematiche del miglioramento delle condizioni e dell'ambiente di lavoro, della protezione della salute e sicurezza dei lavoratori, privilegiando gli scambi di informazioni e gli «approcci innovativi».

La sensazione è, in definitiva, che si tenda a sostenere, in particolare in questa materia, la diffusione di soluzioni e criteri che siano di completamento e perfezionamento del tradizionale sistema di norme rigide. Ciò, tuttavia, non senza innescare dubbi ed interrogativi in ordine alle implicazioni giuridiche dell'uso di queste regole.

Nonostante il «respiro europeo» ${ }^{12}$ infuso nel nostro ordinamento dal rinvio alle buone prassi, nonché alle ulteriori "norme volontarie" (quali i codici di condotta), occorre, come anticipato, che le nuove fonti si concilino con la disposizione di chiusura del sistema di prevenzione dei rischi, l'art. 2087 c.c.: sicché, nihil sub sole novum?

Le regole in tal modo introdotte ed adottate, quali metodi che permettono la risoluzione di problemi, hanno indubbiamente ricadute giuridiche. Del resto, non si può non ricordare che «la posizione di una norma non è mai innocua: essa esprime una libertà che si perde nel momento stesso in cui si esercita $\gg^{13}$.

12 L. Montuschi, Verso il testo unico sulla sicurezza del lavoro, in DLRI, 2007, p. 805.

13 N. IRTI, Due temi di governo societario (responsabilità «amministrativa»codici di autodisciplina), in G. Comm., 2003, p. 699, il quale, riferendosi in particolare ai codici etici e di comportamento, precisa come le società in tal modo «normativizzino scelte e decisioni d'impresa»: i codici etici «si convertono in codici giuridici». 



\section{CAPitolo I}

\section{LE BUONE PRASSI IN MATERIA DI SALUTE E SICUREZZA NEI LUOGHI DI LAVORO}

Sommario: 1. Le buone prassi nel d.lgs. n. 81/2008. - 2. La nozione legislativa e il raccordo con l'art. 2087 c.c. - 3. Il ruolo degli organismi paritetici. - 4. L'atto di validazione. -5 . Contenuto e diffusione delle good practices a livello interno e dell'Unione europea. - 6. Inquadramento e qualificazione giuridica. - 6.1. Le buone prassi quali atti unilaterali integrativi del contratto di lavoro. -7 . Volontarietà e necessità. - 7.1. Quale incidenza sui profili di responsabilità? Alcune ipotesi possibili. - 8. Spunti per una comparazione. - 8.1. L'esperienza spagnola, francese e tedesca. - 8.2. Gli Approved Codes of Practice e le guidances nell'ordinamento britannico.

\section{Le buone prassi nel d.lgs. n. 81/2008}

Tra le novità di non poco rilievo contenute nel d.lgs. n. $81 / 2008$, soprattutto alla luce di un breve confronto comparatistico, v'è la regolamentazione delle buone prassi aziendali, definite quali «soluzioni organizzative o procedurali coerenti con la normativa vigente e con le norme di buona tecnica, adottate volontariamente e finalizzate a promuovere la salute e sicurezza sui luoghi di lavoro attraverso la riduzione dei rischi e il miglioramento delle condizioni di lavoro», elaborate e raccolte dalle Regioni, dall'Inail e dagli organismi paritetici, validate dalla Commissione consultiva permanente per la salute e sicurezza sul lavoro (cfr. l'art. 2, comma 1, lett. v). 
È stato, in tal modo, recepito il criterio direttivo dettato dall'art. 3, comma 1, lettera h, 1. n. 229/2003, poi ripreso dall'art. 1, comma 2, lettera 1, 1. n. 123/2007, che ha promosso la diffusione di buone prassi, così da «orientare verso la ricerca di un ottimale livello di protezione tutti i soggetti coinvolti nell'attuazione del progetto prevenzionale» ${ }^{1}$; rientrano, infatti, tra le misure generali «opportune per garantire il miglioramento nel tempo dei livelli di sicurezza» (art. 15, comma 1, lett. t, d.lgs. n. 81/2008).

L'obiettivo è quello di introdurre soluzioni di tipo organizzativo, procedurale, nonché tecnico, secondo i chiarimenti forniti dalla Commissione consultiva permanente ${ }^{2}$, elevando gli standard di protezione definiti legislativamente; del resto la cultura della sicurezza non è esclusivamente perseguibile attraverso norme di legge.

Il concetto di buone prassi era, per la verità, già diffuso a livello europeo e presente in particolare nel Libro Verde del 2001, dal titolo «Promuovere un quadro europeo per la responsabilità sociale delle imprese». Ivi è precisato che tradizionalmente «i temi della salute e della sicurezza nel lavoro sono stati prevalentemente affrontati attraverso misure legislative e coercitive». Si assiste, tuttavia, ad un interesse sempre maggiore da parte delle imprese, dei governi e delle organizzazioni professionali nei confronti di «forme complementari» di promozione della sicurezza dei prestatori di lavoro, ossia di misure integrative della legislazione che «tendono a sviluppare una cultura della prevenzione, in altre parole un migliore livello di sicurezza e di protezione della salute sul luogo di lavoro» ${ }^{3}$.

Ciò è confermato nella Comunicazione della Commissione delle Comunità Europee relativa alla «strategia comunitaria per la salute e la sicurezza 2002-2006», il cui carattere innovativo si riscontra nel consolidamento di una cultura della prevenzione dei rischi e nella «combinazione di strumenti strategici differenziati», quali la legi-

1 L. Montuschi, Aspettando la riforma: riflessioni sulla legge n. 229 del 2003 per il riassetto in materia di sicurezza sul lavoro, in ADL, 2004, p. 768 ss.

2 Cfr. in particolare il modello per la presentazione delle buone prassi al fine della loro validazione, ai sensi degli artt. 2 e 6, d.lgs. n. 81/2008, approvato dalla Commissione consultiva permanente nella riunione del 20 ottobre 2010.

3 Cfr. il Libro Verde, Promuovere un quadro europeo per la responsabilità sociale delle imprese, presentato dalla Commissione delle Comunità Europee, Bruxelles, 18.7.2001. 
slazione, il dialogo sociale, la spinta al progresso e l'individuazione delle pratiche migliori ${ }^{4}$.

Di non poca rilevanza è, inoltre, il rapporto finale di valutazione della strategia europea sulla sicurezza e la salute sul lavoro (20072012), che si sofferma approfonditamente in merito alla sensibilizzazione alle buone pratiche, atte a supportare l'implementazione, la comprensione e l'applicazione della legislazione in materia ${ }^{5}$. Si diffonde, in tal senso, un «approccio mirato a fornire il sistema di strumenti di autoregolamentazione per una maggiore effettività dello stesso» ${ }^{6}$.

Una più recente conferma si rinviene anche nella Comunicazione della Commissione europea relativa ad un «quadro strategico dell'UE in materia di salute e sicurezza sul lavoro 2014-2020». Tra le «azioni» da intraprendere a partire dal 2014 risultano l'identificazione e lo scambio di good practices, in particolare nelle piccole e micro imprese, al fine di migliorare le condizioni di salute per specifiche categorie di lavoratori, quali i più anziani ed i più giovani inesperti; accanto ad un nucleo legislativo che assicuri un elevato livello di protezione e la creazione di condizioni di parità tra le imprese, indipendentemente dalla loro dimensione, ubicazione o settore di attività, è, pertanto, promossa l'utilizzazione di strumenti «non legislativi», in considerazione anche della «necessità di adottare misure strategiche mirate ed efficaci» ${ }^{7}$.

Promanano dall'Europa, detentrice di un «ruolo fondamentale

4 Cfr. Communication from the Commission - Adapting to change in work and society: a new Community strategy on health and safety at work 2002-2006, Bruxelles, 11.03.2002.

5 Evaluation of the European Strategy on safety and health at work 20072012. Final Report, 15.03.2013, in http://ec.europa.eu. Cfr., al riguardo, L. TRIA, Il difficile raggiungimento dell'obiettivo del benessere organizzativo nel lavoro, in http://www.europeanrights.eu.

6 B. CAruso, L'Europa, il diritto alla salute e l'ambiente di lavoro, in L. Monтuschi (a cura di), Ambiente, Salute e Sicurezza. Per una gestione integrata dei rischi da lavoro, Torino, Giappichelli, 1997, p. 8 ss.

7 Communication from the Commission to the European Parliament, the Council, the European Economic and Social Committee and the Committee of the Regions on an EU Strategic Framework on Health and Safety at Work 2014-2020, Bruxelles, 6.06.2014, in http://ec.europa.eu. Cfr. al riguardo L. Angelini, La sicurezza del lavoro nell'ordinamento europeo, in G. NATULlo (a cura di), Salute e sicurezza sul lavoro, Milano, Utet Giuridica, 2015, p. 103. 
nella promozione della salute e della sicurezza nei luoghi di lavoro» ${ }^{8}$, gli stimoli ad accostare ed armonizzare fonti diverse in questa tematica divenuta centrale nella politica sociale dell'Unione europea. È opportuno, pertanto, anche a livello interno prendere in considerazione l'apertura verso tali regole, forse alquanto sottostimate nella riflessione giuridica ed avvicinate per molto tempo con cautela, se non con sospetto, dalle parti sociali e dalle stesse imprese.

Fino alla regolamentazione ad opera del d.lgs. n. 81/2008, nel nostro ordinamento i riferimenti alle buone prassi erano del tutto marginali e per lo più contenuti in alcune leggi regionali, ove tuttavia le stesse non erano esattamente definite né sottoposte a criteri di validazione; era promossa la loro diffusione al fine di orientare «i comportamenti dei datori di lavoro e dei lavoratori verso il rispetto delle norme ed il miglioramento degli standard di salute e sicurezza» ${ }^{9}$.

Sicché è solo con il d.lgs. n. 81/2008 che le buone prassi risultano pienamente configurate.

Nel decreto sono presenti diversi rinvii: rileva in proposito l'art. 35 che promuove l'individuazione di codici di comportamento e di buone prassi in occasione della riunione periodica, alla quale partecipano, oltre al datore di lavoro, il responsabile del servizio di prevenzione e protezione, il medico competente e il rappresentante dei lavoratori per la sicurezza. Con cadenza annuale e solo per le imprese soggette a tale obbligo, è in tal modo conferita la possibilità di introdurre soluzioni coerenti con le previsioni normative, al fine di prevenire i rischi di infortuni e di malattie professionali. Il momento della riunione periodica, tradizionalmente deputato all'analisi del documento di valutazione dei rischi, dell'idoneità dei mezzi di prote-

8 Si veda P. Tullini, Sicurezza sul lavoro e modello sociale europeo: un'ipotesi di sviluppo, in Scritti in onore di Edoardo Ghera, Bari, Cacucci, 2008, II, p. 1257.

9 In tal senso cfr. la legge regionale Liguria del 13 agosto 2007, n. 30; si veda, inoltre, la legge regionale Emilia-Romagna del $1^{\circ}$ agosto 2005, n. 17, contenente «Norme per la promozione dell'occupazione, della qualità, sicurezza e regolarità del lavoro», che ha disposto che la Regione e le Province sostengano interventi di informazione e pubblicizzazione delle buone prassi e delle esperienze realizzate. Per un esame della legislazione regionale cfr. A. Morrone, Art. 1, in C. Zoli (a cura di), Principi comuni, in L. Montuschi (dir.), La nuova sicurezza sul lavoro, Bologna, Zanichelli, 2011, vol. I, p. 50 ss. 
zione individuale, dei programmi di informazione e formazione, acquista dunque, da questo punto di vista, un valore aggiunto.

Attraverso espliciti richiami del legislatore le buone prassi divengono, altresì, parametri di riferimento dell'adempimento dell'obbligo di sicurezza rispetto all'esposizione a determinati rischi.

È, infatti, previsto che il datore di lavoro, con riguardo all'uso delle attrezzature di lavoro, provveda «al fine di assicurarne l'installazione corretta e il buon funzionamento», secondo le indicazioni fornite dalle buone prassi, oltreché dalle pertinenti norme tecniche o dalle linee guida (art. 71, comma 8) ${ }^{10}$.

Quanto alla movimentazione manuale dei carichi, nell'adempimento degli obblighi datoriali si può fare riferimento anche alle buone prassi (art. 168).

Più specificamente, nell'ambito della valutazione dei rischi derivanti da esposizione ad agenti fisici il datore di lavoro dovrà prendere in esame le norme di buona tecnica e le buone prassi, «in modo da identificare e adottare le opportune misure di prevenzione e protezione» (art. 181, comma 1$)^{11}$.

Deve essere, altresì, segnalata l'interessante previsione contenuta nel Titolo XII dello stesso d.lgs. n. 81/2008, che ha sancito in capo agli organi di vigilanza il «potere di disposizione», ai fini dell'applicazione, oltreché delle norme tecniche, delle buone prassi, volon-

10 Ai sensi dell'art. 71, comma 8, lett. b, d.lgs. n. 81/2008, le attrezzature soggette a influssi che possono provocare deterioramenti suscettibili di dare origine a situazioni pericolose sono sottoposte «ad interventi di controllo periodici, secondo frequenze stabilite in base alle indicazioni fornite dai fabbricanti, ovvero dalle norme di buona tecnica, o in assenza di queste ultime, desumibili dai codici di buona prassi».

11 Cfr., inoltre, l'art. 209, d.lgs. n. 81/2008, modificato dal d.lgs. 1 agosto 2016, n. 159, che prevede la valutazione, misurazione ed il calcolo dell'esposizione dei lavoratori ai campi elettromagnetici, tenendo anche conto «delle specifiche buone prassi individuate o emanate dalla Commissione consultiva permanente»; nonché l'art. 216, d.lgs. n. 81/2008, riguardante l'esposizione dei lavoratori a radiazioni ottiche artificiali, ai sensi del quale il datore di lavoro adotta le buone prassi individuate o emanate dalla Commissione consultiva permanente nelle situazioni che esulano dalle norme della Commissione elettrotecnica internazionale (IEC), per quanto riguarda le radiazioni laser, e dalle raccomandazioni della Commissione internazionale per l'illuminazione (CIE) e del Comitato europeo di normazione $(\mathrm{CEN})$, per quanto riguarda le radiazioni incoerenti, fino a quando non saranno disponibili norme e raccomandazioni adeguate dell'Unione europea. 
tariamente impiegate ed espressamente richiamate dal datore di lavoro in sede ispettiva, «qualora ne riscontrino la non corretta adozione, e salvo che il fatto non costituisca reato» (art. 302-bis, d.lgs. n. 81/2008).

Condizioni per la soggezione a questo non meglio precisato potere da parte degli organi ispettivi di impartire disposizioni esecutive in ordine all'utilizzo delle buone prassi sono, appunto, la volontarietà della loro adozione, nonché il loro esplicito richiamo. Da tale manifestazione di volontà discende un duplice obbligo: per il personale di vigilanza di valutare la «corretta adozione» delle buone prassi segnalate, per il datore di lavoro di non potersi sottrarre a questo giudizio. Come sancito dall'art. 302-bis, comma 2, avverso le disposizioni è, però, ammesso ricorso entro trenta giorni, con eventuale richiesta di sospensione dell'esecutività dei provvedimenti, all'autorità gerarchicamente sovraordinata nell'ambito dei rispettivi organi di vigilanza.

Tale controllo legislativamente previsto ed esercitabile da parte del personale ispettivo non può, dunque, essere ignorato da parte del datore di lavoro' ${ }^{12}$.

A dire il vero, la previsione citata non fornisce alcun chiarimento sulle conseguenze dell'eventuale mancato rispetto della disposizione, che resta non legislativamente sanzionato. Tuttavia, trattandosi di disposizioni esecutive, paragonabili a quelle già disciplinate dal d.p.r. 19 marzo 1955, n. 520, pare opportuno richiamare l'art. 11, comma 2, ivi contenuto, che regolamenta le inosservanze delle disposizioni legittimamente impartite dagli ispettori nell'esercizio delle loro funzioni in materia di sicurezza o igiene del lavoro ${ }^{13}$. Il d.p.r. n. 520/1955, non rientrante tra le abrogazioni espresse, di cui all'art. 304, d.lgs. n. 81/2008, si può ritenere tuttora in vigore, in quanto non abrogato nemmeno implicitamente.

12 In proposito cfr. P. Pascucci, A. Andreani, L'estinzione agevolata degli illeciti amministrativi e il potere di disposizione, in L. Zoppoli, P. PAScucci, G. NATULLo (a cura di), Le nuove regole per la salute e la sicurezza dei lavoratori, Milano, Ipsoa, 2010, p. 658 ss.

13 Ai sensi dell'art. 11, comma 2, d.p.r. n. 520/1955, la pena è quella dell'arresto fino a un mese o dell'ammenda fino a 413 euro. In tal senso cfr. F. MALZANI, Ambiente di lavoro e tutela della persona. Diritti e rimedi, Milano, Giuffrè, 2014, pp. 226 e 235. 
Non va, piuttosto, sottovalutato che l'organo di vigilanza nell'esercitare il proprio potere deve possedere un'adeguata preparazione ed esperienza in merito alla corretta attuazione degli strumenti menzionati ${ }^{14}$. Le buone prassi sono, dunque, passibili di una successiva verifica, da considerarsi vincolante, al pari del più complesso e onnicomprensivo obbligo di prevenzione.

\section{La nozione legislativa e il raccordo con l'art. 2087 c.c.}

Il menzionato art. 2, comma 1, lett. v, d.lgs. n. 81/2008, per quanto scarno ed essenziale, costituisce invero la disposizione cui rivolgere maggiormente l'attenzione. Ruoli di particolare importanza sono ivi attribuiti alle Regioni, all'Inail ${ }^{15}$, agli organismi paritetici, nonché alla Commissione consultiva permanente. Sono, così, individuati una serie di enti pubblici ed organismi cui la legge ha affidato specifiche funzioni.

L'elaborazione e la raccolta delle buone prassi è compito delle Regioni, dell'Inail e degli organismi paritetici, disciplinati dall'art. 51, d.lgs. n. 81/2008. È, in particolare, l'Inail ad aver acquisito, con il riassetto e la riforma delle norme vigenti, un ruolo arricchito, concorrendo alla prevenzione dei rischi, alla promozione e divulgazione della cultura della salute ed alla garanzia di un miglior livello di sicurezza nei luoghi di lavoro. Da questo punto di vista, spetta all'Inail raccogliere e mettere a disposizione delle imprese i metodi e le soluzioni più innovative al fine del miglioramento delle condizioni di lavoro.

Strettamente connesse sono le «attività promozionali», di cui all'art. 11, d.lgs. n. 81/2008, ossia il finanziamento da parte dell'Inail di progetti, in particolare volti a sperimentare «strumenti di natura organizzativa e gestionale ispirati ai principi di responsabilità sociale», costituendo «criterio di priorità» l'adozione delle buone

14 In proposito cfr. P. Pascucci, A. Andreani, L'estinzione agevolata degli illeciti amministrativi e il potere di disposizione, cit., p. 663.

15 Si veda la legge 30 luglio 2010, n. 122 di conversione con modificazioni del d.l. n. 78/2010, che ha previsto l'attribuzione all'Inail delle funzioni già svolte in materia dall'Ispels. 
prassi $^{16}$. Questi incentivi economici consentono, così, di «incidere virtuosamente sul rapporto tra costi e risultati della prevenzione nei luoghi di lavoro» ${ }^{17}$.

Il legislatore, come chiarito, ha tenuto in grande considerazione anche le Regioni, variamente coinvolte nel sistema normativo del diritto della sicurezza del lavoro, con funzioni di indirizzo e coordinamento, di carattere organizzativo e gestionale, di informazione e di promozione, di vigilanza, nonché regolative ${ }^{18}$. A queste ultime può in parte essere ricondotta l'elaborazione e la raccolta delle buone prassi ${ }^{19}$.

Finora questo compito è stato adempiuto da alcune Regioni, che hanno anche provveduto ad adottare proprie leggi, formalizzando l'intenzione di promuovere, d'intesa con gli organismi bilaterali, se costituiti, o con le parti sociali, buone prassi, oltreché codici etici ed accordi aziendali, favorendone lo scambio e la condivisione, per innalzare gli standard di tutela dei prestatori di lavoro ${ }^{20}$. Tali interventi

16 Occorre precisare che l'Inail mette a disposizione delle imprese sia forme di sostegno economico per la realizzazione di progetti volti al «miglioramento dei livelli di salute e sicurezza nei luoghi di lavoro» (cfr. l'iniziativa ISI promossa da ultimo nel 2016), sia la possibilità di riduzione del premio assicurativo in seguito ad interventi già effettuati in aggiunta a quelli previsti per legge (cfr. l'iniziativa OT24). Sono, inoltre, contemplate forme di finanziamento nei confronti delle piccole e micro imprese operanti nei settori dell'agricoltura, dell'edilizia, dell'estrazione e della lavorazione dei materiali lapidei per la realizzazione di progetti di innovazione tecnologica mirati al miglioramento delle condizioni di salute e sicurezza nei luoghi di lavoro (cfr. il bando Fipit 2014): www.inail.it.

17 G. Natullo, Il quadro normativo dal Codice civile al Codice della sicurezza sul lavoro. Dalla Massima sicurezza (astrattamente) possibile alla Massima sicurezza ragionevolmente (concretamente) applicata?, in ID. (a cura di), Salute e sicurezza sul lavoro, cit., p. 21.

18 G. Natullo, I ruoli delle Regioni nel disegno del d.lgs. n. 81/2008, in P. Pascucci (a cura di), Il d.lgs. n. 81/2008: due anni dopo. I "sistemi" del diritto della sicurezza sul lavoro, Atti dell'incontro di studio di Urbino, 14-15 maggio 2010, Quaderni di Olympus-2, 2011, http://olympus.uniurb.it/, p. 108 ss.

19 Si veda, ad esempio, l'accordo di collaborazione del 25 gennaio 2012, tra Inail, Direzione regionale Emilia-Romagna e Regione Emilia-Romagna, «per incentivare l'utilizzo del registratore delle presenze autorizzate nei cantieri (REPAC)», ove è esplicitato l'obiettivo condiviso di «sperimentare soluzioni pratiche che favoriscano e/o premino le azioni per la prevenzione e contribuiscano a diffondere la cultura della salute e sicurezza».

20 Cfr., ad esempio, la legge della Regione Puglia del 10 marzo 2014, n. 8, recante «Norme per la sicurezza, la qualità e il benessere sul lavoro», che ha sotto- 
legislativi, per la verità piuttosto generici e riferentisi prevalentemente alla responsabilità sociale d'impresa, sono però supportati dalla specifica previsione di incentivi economici. Le menzionate previsioni normative, pur nella difficile e assai dibattuta convivenza della competenza legislativa statale e regionale in subiecta materia, sembrano integrare il quadro legislativo nazionale senza creare interferenze ${ }^{21}$.

Si deve precisare che la definizione legislativa di buone prassi, dettata all'art. 2, comma 1, lett. v, d.lgs. n. 81/2008, che si riferisce a prassi condivise ed attuate volontariamente, nonché validate dalla Commissione consultiva permanente al fine di promuovere la salute dei prestatori di lavoro, suscita, in verità, alcuni delicati interrogativi sui quali è necessario soffermarsi.

Le soluzioni organizzative e procedurali devono, in primo luogo, essere «coerenti con la normativa vigente» ossia con le previsioni contenute nello stesso decreto n. 81, nonché con l'art. 2087 c.c., disposizione che, pur «appartenente ad una stagione economico-so-

lineato l'importanza del perseguimento della cultura della salute e della sicurezza sul lavoro «quale parte integrante della qualità dell'azione delle proprie politiche dello sviluppo economico e sociale e delle politiche di tutela del lavoro» e promosso «azioni di prevenzione dei rischi e di miglioramento continuo delle condizioni di lavoro, anche attraverso la stipula di accordi territoriali e settoriali con gli enti locali, le parti sociali e gli enti istituzionali competenti in materia», nonché la divulgazione di buone pratiche trasferibili sul territorio regionale; la legge della Regione Campania del 9 agosto 2013, n. 11, in linea con la precedente legge regionale del 18 novembre 2009 , n. 14 , che ha stabilito l'erogazione di contributi finanziari alle imprese «per interventi diretti al miglioramento dei livelli di sicurezza del lavoro e della qualità degli ambienti di lavoro»; la legge della Regione Emilia-Romagna n. 2/2009, in merito alla «Tutela e sicurezza del lavoro nei cantieri edili e di ingegneria civile», che ha inteso incentivare livelli ulteriori di sicurezza, tra i quali l'utilizzo di sistemi informatici telematici per la verifica delle presenze autorizzate nei cantieri; la legge della Regione Piemonte, n. 34/2008, in tema di «Norme per la promozione dell'occupazione, della qualità, della sicurezza e regolarità del lavoro».

21 Cfr. in particolare sul tema P. TULLINI, Le competenze legislative in materia di sicurezza sul lavoro: i nodi irrisolti, in Le Istituzioni del Federalismo, 2009, suppl. n. 1, p. 15 ss.; EAD., Legge e legislazione lavoristica, in M. PERSIANI (a cura di), Le fonti del diritto del lavoro, Trattato di diritto del lavoro, diretto da M. Persiani e F. Carinci, Padova, Cedam, 2010, p. 464; A. TroJsi, La potestà legislativa dello Stato e delle Regioni, in L. Zoppoli, P. Pascucci, G. Natullo (a cura di), Le nuove regole per la salute e la sicurezza dei lavoratori, cit., p. 15 ss.; V. SPEZiale, La nuova legge sulla sicurezza del lavoro, in WP C.S.D.L.E. "Massimo D'Antona" .IT- 60/2007, p. 6. 
ciale e giuridica che non esiste più̀ ${ }^{22}$, continua a rivestire un ruolo centrale in materia.

Viene, allora, in rilievo l'esatto raccordo tra le buone prassi e l'art. 2087 c.c.

La questione è senza dubbio complessa e diverse potrebbero essere le opzioni interpretative.

Il problema del raccordo nasce dalla stessa definizione legislativa che sottolinea il carattere volontario delle misure migliorative delle condizioni di lavoro adottate. Sul presupposto che la buona prassi sorga per far fronte ad una esigenza di sicurezza ci si deve domandare se la «volontaria adozione» della misura non confligga con l'obbligo di sicurezza di cui all'art. 2087 c.c.

Il legislatore ha fatto riferimento a soluzioni conformi alla normativa, senza tuttavia precisare se debba trattarsi di misure solo specificative di quanto disposto nel d.lgs. n. 81/2008 ovvero realmente aggiuntive ed ulteriori; quid iuris, inoltre, rispetto all'art. 2087 c.c.?

In verità occorre premettere che, con riguardo alla casistica, pare trattarsi di soluzioni dal contenuto vario, come si cercherà di chiarire, a volte del tutto innovative e costruite su misura della specifica situazione di rischio, altre volte semplicemente di supporto all'adempimento degli obblighi previsti dal d.lgs. n. 81/2008 (cfr. in particolare il paragrafo 5).

Quanto all'art. 2087 c.c. v'è brevemente da ricordare che l'indirizzo ormai consolidato della giurisprudenza ha rimarcato il contenuto non specificato ad esso sotteso, cui consegue l'assoggettamento e la permeabilità ai progressi della scienza e della tecnica; dunque, la norma definibile «in bianco» è destinata ad essere riempita anche attraverso «misure di sicurezza innominate» ossia non espressamente previste dalla legge ed ancorate alla particolarità del lavoro e alla natura dell'ambiente di lavoro ${ }^{23}$. Misure, tuttavia, concretamente individuate, non essendo imposta l'adozione

22 L. Montuschi, Dai principi al sistema della sicurezza sul lavoro, in ID. (dir.), La nuova sicurezza sul lavoro, cit., p. 9.

23 Cass., sez. lav., 13 giugno 2017, n. 14665, in www.olympus.uniurb.it; Cass., sez. lav., 5 gennaio 2016, n. 34, in DeJure; Cass., sez. lav., 8 aprile 2013, n. 8486, in DeJure; Cass., sez. lav., 25 maggio 2006, n. 12445, in RIDL, 2007, II, p. 66. 
di ogni cautela possibile «diretta ad evitare qualsiasi danno» ${ }^{24}$. Se ne deduce l'obbligo in capo al datore di lavoro di predisporre le misure di «prudenza e diligenza» e di osservare le «norme tecniche e di esperienza $»^{25}$. L'individuazione delle cautele deve essere, così, parametrata al criterio della diligenza «qualificata» richiesta nell'adempimento dell'obbligo di sicurezza ai sensi dell'art. 1176, comma 2 , c.c. ${ }^{26}$.

V'è allora da chiedersi se tra i comportamenti, suggeriti dalle conoscenze sperimentali e tecniche, doverosi al fine di scongiurare il rischio di infortuni e malattie professionali, possano essere ricomprese le richiamate buone prassi. Si deve, quindi, considerare la reciproca influenza tra la disciplina (speciale) inclusa nel d.lgs. n. $81 \mathrm{e}$ la norma generale di cui all'art. 2087 c.c.; tale «interazione», come sottolineato, «può restituire una corretta configurazione dell'obbligo di sicurezza ${ }^{27}$.

Con riferimento alle singole tipologie di prassi occorre, in particolare, valutare se la volontarietà richiamata nella definizione di cui all'art. 2, comma 1, lett. v, d.lgs. n. 81, sia riferibile all'an dell'adozione o piuttosto al quomodo, dunque alle modalità di adempimento dell'obbligo di sicurezza. Posto l'obiettivo di perseguire la massima sicurezza in azienda, insito nell'art. 2087 c.c., la discrezionalità la-

24 Cass., sez. lav., 2 giugno 1998, n. 5409, in DeJure; Cass., sez. lav., 12 luglio 2004, n. 12863, in DeJure; Cass., sez. lav., 1 giugno 2004, n. 10510, in DeJure, ove è sancita la sussistenza della responsabilità datoriale nel caso siano violati «gli obblighi di comportamento suggeriti dalle conoscenze sperimentali e tecniche del momento»; Cass., sez. lav., 2 luglio 2014, n. 15082, in DeJure; Cass., sez. lav., 2 settembre 2015, n. 17438, in DeJure.

25 Cass. 9 maggio 1998, n. 4721, in GC, 1999, I, p. 539; Cass., sez. lav., 17 febbraio 2009, n. 3785, in DeJure; Cass., sez. lav., 17 aprile 2012, n. 6002, in DeJure.

26 C. Smuraglia, La sicurezza del lavoro e la sua tutela penale, Milano, Giuffrè, 1974, p. 79; cfr., inoltre, al riguardo L. Montuschi, La Corte Costituzionale e gli standard di sicurezza del lavoro, in $A D L, 2006$, n. 1, p. 13. Per una indagine storica in ordine all'obbligo contrattuale di garantire la sicurezza del lavoro cfr. L. GAETA, Infortuni sul lavoro e responsabilità civile. Alle origini del diritto del lavoro, Napoli, Edizioni Scientifiche Italiane, 1986, p. 59 ss.

27 P. Albi, La sicurezza sul lavoro e la cultura giuridica italiana tra vecchio e nuovo diritto, in DSL, 2016, n. 1, p. 88; nonché ID., Adempimento dell'obbligo di sicurezza e tutela della persona, in Il Codice Civile, Commentario fondato da P. Schlesinger, diretto da F.D. Busnelli, Milano, Giuffrè, 2008, p. 82. 
sciata al datore di lavoro potrebbe, infatti, riguardare la scelta della soluzione più appropriata o confacente alla situazione di rischio prefigurata.

Da questo punto di vista, si potrebbe ritenere che le buone prassi, quali strumenti individuati dal legislatore per orientare la condotta del datore di lavoro, traggano la loro origine dall'esistenza di un problema di sicurezza da risolvere ed offrano una possibile strada risolutiva, ma non l'unica; una possibile modalità per fronteggiare il rischio a fronte di un problema che il datore di lavoro non può ignorare.

Ad arricchire e complicare le ipotesi fin qui formulate v'è poi la considerazione che accanto a prassi già "confezionate", quindi validate e trasferibili in diversi contesti lavorativi, residua la possibilità, non infrequente, di sperimentazione di soluzioni ad hoc, non validate. Il panorama delle soluzioni tecniche, organizzative o procedurali intraprese in corrispondenza di specifici rischi si presenta, infatti, molto ampio e non necessariamente collegato ad una apposita validazione, come invece richiesto nel d.lgs. n. $81 / 2008$.

Come risolvere, dunque, il problema del raccordo con l'art. $2087 ?$

Il quesito attiene alla astratta configurabilità di soluzioni realmente aggiuntive rispetto allo stesso disposto dell'art. 2087 c.c.; soluzioni cioè non «necessarie» a tutelare la salute dei lavoratori e liberamente perseguibili dal datore di lavoro. Misure che rimanendo fuori dall'obbligo di sicurezza, sarebbero appunto contraddistinte da una vera facoltatività $a b$ origine quanto alla loro stessa adozione. In caso contrario, si prefigura la possibilità che le buone prassi siano considerate strumenti per il corretto adempimento del dovere di sicurezza e disvelino un obbligo già esistente, perdendo conseguentemente la caratteristica della volontarietà, pur attribuita loro dalla legge.

Si prospettano, in sostanza, diversi nodi da sciogliere, a partire dalla efficacia dell'atto di validazione e dalla qualificazione giuridica attribuibile alle buone prassi.

Nonostante il d.lgs. n. 81/2008 non contempli espressamente alcuna sanzione in ipotesi di mancata attuazione delle buone pras- 
si, di cui è, appunto, sancita la natura volontaria, v'è in ogni caso da interrogarsi in ordine ai possibili profili di responsabilità datoriale che ne derivano.

\section{Il ruolo degli organismi paritetici}

Nella direzione di sostenere e valorizzare l'individuazione di soluzioni finalizzate a garantire ed elevare la protezione durante il lavoro si collocano, accanto ai già menzionati soggetti istituzionali della prevenzione, anche gli organismi paritetici, costituiti a iniziativa di una o più associazioni dei datori e dei prestatori di lavoro «comparativamente più rappresentative sul piano nazionale», quali sedi privilegiate di elaborazione e raccolta di buone prassi a fini prevenzionistici (art. 2, comma 1, lett. ee, d.lgs. n. $81 / 2008)$.

Il decreto n. 81/2008 si ispira, anche nella definizione, al d.lgs. n. 276/2003 in materia di occupazione e mercato del lavoro, che già aveva affidato agli enti bilaterali «lo sviluppo di azioni inerenti la salute e la sicurezza sul lavoro» ${ }^{28}$. Legittimate alla costituzione degli organismi paritetici, species degli enti bilaterali in tema di salute e sicurezza sul lavoro, sono però solo le organizzazioni comparativamente più rappresentative «sul piano nazionale»; si tratta, in sostanza, di una delle tante occasioni in cui il legislatore ha operato una selezione ed introdotto il requisito della maggiore rappresentatività comparata a livello nazionale, diversamente da quanto previsto nel ricordato d.lgs. n. 276/200329.

Intorno a questa formula, di incerta interpretazione a causa dell'assenza di indicazioni di fonte legislativa, è sorto da tempo un animato dibattito dottrinale. L'utilizzo del concetto di rappresentatività sindacale è, anche in questa circostanza, finalizzato a con-

28 Cfr., in particolare, l'art. 2, comma 1, lett. h, d.lgs. n. 276/2003.

29 Cfr. sul punto C. Zoli, Art. 51, in ID. (a cura di), Principi comuni, in L. Montuschi (dir.), La nuova sicurezza sul lavoro, cit., p. 530 ss.; nonché C. LazZARI, Gli organismi paritetici nel decreto legislativo 9 aprile 2008, n. 81, in I Working Papers di Olympus, n. 21/2013, p. 2 ss. 
notare quantitativamente e qualitativamente le capacità del sindacato $^{30}$.

Si deve sottolineare che, ai sensi dell'art. 51, comma 4, d.lgs. n. $81 / 2008$, sono fatti salvi gli organismi bilaterali o partecipativi «previsti da accordi interconfederali, di categoria, nazionali, territoriali o aziendali». Da tempo, la tendenza è stata, in effetti, quella di istituire e regolamentare organismi paritetici da parte della contrattazione collettiva a livello interconfederale, in ossequio alla prassi contrattuale invalsa di gestione della sicurezza dei lavoratori nell'ottica della bilateralità ${ }^{31}$. È, però, anche vero che alla regolamentazione ad opera della contrattazione interconfederale non è, poi, corrisposto, sul piano concreto, un reale esercizio dei compiti affidati in tema di prevenzione.

Rispetto alla previgente disciplina, di cui all'abrogato d.lgs. n. $626 / 1994$, il ruolo di questi organismi è stato certamente rafforzato, in ottemperanza alla legge delega n. 123/2007, che aveva sancito, tra gli altri, il criterio della «rivisitazione e potenziamento delle funzioni degli organismi paritetici, anche quali strumenti di aiuto alle imprese nell'individuazione di soluzioni tecniche e organizzative dirette a garantire e migliorare la tutela della salute e sicurezza

30 In proposito le Linee applicative approvate dalla Conferenza Stato-Regioni e Province autonome di Trento e Bolzano del 25 luglio 2012 hanno indicato quale criterio presuntivo della «rappresentatività comparata» la costituzione degli organismi paritetici nell'ambito di «associazioni datoriali o sindacali cui aderiscano organizzazioni datoriali o sindacali - nazionali, territoriali o di settore - firmatarie di un contratto collettivo nazionale di lavoro». Sul punto è intervenuta anche la circolare del Ministero del lavoro e delle politiche sociali del 29 luglio 2011, n. 20, nonché del 5 giugno 2012, n. 13, quest'ultima relativa alla nozione di organismi paritetici nel settore edile. V'è, inoltre, da richiamare il Testo Unico sulla Rappresentanza firmato da Cgil, Cisl, Uil e Confindustria il 10 gennaio 2014, successivamente modificato con l'Accordo del 4 luglio 2017.

31 Si segnalano, da questo punto di vista, alcuni accordi in materia di rappresentanti dei lavoratori per la sicurezza e pariteticità: l'accordo interconfederale Confapi del 20 settembre 2011, in sostituzione dell'accordo del 27 ottobre 1995; l'accordo interconfederale Confartigianato del 13 settembre 2011, in sostituzione dell'accordo del 3 settembre 1996. Cfr., inoltre, l'accordo tra Confindustria Piemonte e Cgil, Cisl e Uil del Piemonte del 23 novembre 2010, con il quale le parti firmatarie si impegnano a far sì che su tutto il territorio regionale sia ripristinata l'operatività degli organismi paritetici provinciali istituiti ai sensi dell'accordo interconfederale Confindustria del 22 giugno 1995. 
sul lavoro» ${ }^{32}$. Tale previsione è stata accolta nell'art. 51 , comma 3 , d.lgs. n. 81, che ne ribadisce la funzione di supporto ed assistenza alle imprese.

L'obiettivo, come sostenuto, è quello di «favorire la prevenzione tramite la partecipazione, ovvero la collaborazione di tutti i soggetti, pubblici e privati» ${ }^{33}$. I concetti, tra di loro affini, di pariteticità e bilateralità richiamano, dunque, la prospettiva di una gestione condivisa e partecipata della sicurezza, che resta tuttavia nell'ombra all'interno del sistema di relazioni industriali italiano, tuttora contraddistinto da una «scarsa diffusione della cultura della bilateralità» ${ }^{34}$. Occorrerebbe, allora, nuovamente invocare il «superamento della dimensione conflittuale e negoziale, considerata come esclusiva e non solo prevalente per l'organizzazione sindacale, mediante l'affermazione della cultura della partecipazione e della gestione» ${ }^{35}$.

Il decreto del 2008, all'art. 2, lett. ee, ha affidato agli organismi paritetici ambiziose funzioni di «programmazione di attività formative», «sviluppo di azioni inerenti alla salute e alla sicurezza sul lavoro», «assistenza alle imprese finalizzata all'attuazione degli adempimenti in materia», nonché, come già ricordato, «elaborazione e raccolta di buone prassi». La previsione, di cui all'art. 52, d.lgs. n. $81 / 2008$, ha, inoltre, contemplato forme di sostegno economico nei confronti della «piccola e media impresa», dei «rappresentanti dei lavoratori per la sicurezza territoriali» e della «pariteticità», attraverso l'istituzione presso l'Inail di un apposito «fondo di sostegno».

Invero, nonostante si stia ampliando la presenza di tali organismi soprattutto a livello provinciale, seppur con preferenza per al-

32 Sul punto cfr. F. BASENGHI, La legge delega in materia di sicurezza: alcuni spunti di indagine, in L. Montuschi (a cura di), Un diritto in evoluzione. Studi in onore di Yasuo Suwa, Milano, Giuffrè, 2007, p. 467.

33 C. Zoli, Art. 51, in ID. (a cura di), Principi comuni, in L. Montuschi (dir.), La nuova sicurezza sul lavoro, cit., p. 529 ss.; cfr., inoltre, ID., Sicurezza del lavoro: contrattazione e partecipazione, in $R G L, 2000$, p. 622, il quale, con riferimento alla disciplina di cui al d.lgs. n. 626/1994, ha sottolineato che gli organismi paritetici erano "privi di penetranti poteri di intervento in via preventiva».

34 M. Ricci, Gli organismi paritetici e il fondo di sostegno, in L. Zoppoli, P. Pascucci, G. Natullo (a cura di), Le nuove regole per la salute e la sicurezza dei lavoratori, cit., p. 521 ss.

35 M. NAPOLI, Gli enti bilaterali nella prospettiva di riforma del mercato del lavoro, in Jus, 2003, p. 237. 
cuni settori, le funzioni da essi esercitate restano in prevalenza circoscritte alla promozione e programmazione dell'attività formativa. Come rimarcato nell'apposito Accordo, in sede di Conferenza Stato Regioni, del 21 dicembre 2011 tra il Ministro del lavoro e delle politiche sociali, il Ministro della salute, le Regioni e le Province autonome di Trento e Bolzano, in ordine alla formazione è, infatti, richiesta in via preventiva la collaborazione degli organismi paritetici territorialmente competenti.

Quanto più esattamente all'innovativo compito di «individuazione e raccolta» di prassi migliorative, come anticipato, almeno ad un primo e rapido esame, sembra che l'esperienza della gestione partecipata della sicurezza attraverso gli organismi bilaterali e paritetici non sia ancora pienamente decollata nel nostro ordinamento; è possibile, tuttavia, riscontrare a livello regionale qualche esperienza degna di nota ${ }^{36}$.

\section{L'atto di validazione}

Deputata alla validazione delle buone prassi è la Commissione consultiva permanente per la salute e la sicurezza sul lavoro, istituita presso il Ministero del lavoro e delle politiche sociali e composta da un rappresentante di alcuni Ministeri ${ }^{37}$, nonché da sei rappresen-

36 È utile richiamare l'esperienza della Regione Veneto nel settore dell'artigianato e, in particolare, l'accordo interconfederale regionale del 31 ottobre 2003 tra Confartigianato del Veneto, Cna del Veneto, Casartigiani del Veneto e Cgil Veneto, Cisl Veneto, Uil Veneto, che ha istituito un modello di rappresentanza autonomo. Il Comitato paritetico bilaterale regionale per la sicurezza si distingue, infatti, per la propria indipendenza rispetto all'Ente bilaterale del Veneto (EBAV), sviluppando politiche autonome in materia di sicurezza. Tra le diverse funzioni ad esso attribuite si ricordi quella «consultiva», che comprende l'indicazione di misure migliorative delle condizioni di lavoro. Sul punto cfr. S. Bellumat, La gestione della salute e della sicurezza, in L. Nogler (a cura di), Ebav. Uno strumento delle parti sociali al servizio dell'artigianato veneto, Franco Angeli, Milano, 2014, p. 301 ss. In merito agli enti bilaterali regionali dell'artigianato cfr. L. NoGLER (a cura di), Gli enti bilaterali dell'artigianato tra neo-centralismo ed esigenze di sviluppo, Milano, Franco Angeli, 2014.

37 In particolare si tratta di un rappresentante del Ministero del lavoro e delle politiche sociali (con funzioni di presidente), del Ministero della salute, del Ministe- 
tanti delle Regioni e delle Province Autonome di Trento e di Bolzano individuati dalla Conferenza permanente per i rapporti tra lo Stato, le Regioni e le Province Autonome, da sei esperti designati delle organizzazioni sindacali dei lavoratori e dei datori di lavoro comparativamente più rappresentative a livello nazionale, da tre esperti in medicina del lavoro, igiene industriale e impiantistica industriale, da un rappresentante dell'ANMIL (art. 6, d.lgs. n. 81/2008, come modificato dal d.lgs. n. 151/2015).

Alla Commissione, già presente nell'abrogato d.lgs. n. 626/1994, ma rivisitata quanto alla composizione ed ai compiti nel d.lgs. n. $81 / 2008$, è stata, in particolare, attribuita maggiore rilevanza nella «promozione della cultura della prevenzione» ${ }^{38}$, essendole appunto affidate, tra le altre, funzioni quali la definizione delle attività di promozione e le azioni di prevenzione di cui all'art. 11, d.lgs. n. $81 / 2008$, la valorizzazione degli accordi sindacali e dei codici di condotta ed etici, nonché la validazione delle buone prassi; in proposito, è stato appositamente istituito al suo interno un comitato speciale permanente, ai sensi dell'art. 6, comma 3 del medesimo decreto.

Tra i documenti finora approvati dalla Commissione consultiva permanente con specifico riguardo alla problematica de qua v'è il menzionato modello di presentazione delle buone prassi, la cui precisa e completa compilazione consente di avviare la procedura di selezione e validazione.

Il modello, possibilmente corredato da ulteriore documentazione di supporto, si rifà in parte alle informazioni relative alle buone

ro dello sviluppo economico, del Ministero delle infrastrutture e dei trasporti, del Ministero dell'interno, del Ministero della difesa, del Ministero delle politiche agricole alimentari e forestali, nonché un rappresentante del Ministero dell'istruzione, dell'università e della ricerca o un rappresentante della Presidenza del Consiglio dei Ministri - Dipartimento della funzione pubblica quando il presidente della Commissione, ravvisando profili di specifica competenza, ne disponga la convocazione.

38 P. PASCUCCI, La tutela della salute e della sicurezza sul lavoro: il titolo I del d.lgs. n. 81/2008, Quaderni di Olympus-4, Fano, Aras Edizioni, 2014, p. 83 ss., secondo il quale la Commissione «ha un volto meno regolativo che in passato e più incline ad una dimensione promozionale», pur non essendo scomparsi i compiti più strettamente tecnici. Sul tema cfr. O. Bonard, Dentro al ginepraio della semplificazione. Salute e sicurezza sul lavoro nell'Italia che riparte, in RGL, 2016, n. 3, p. 605 , la quale definisce la citata Commissione come «vera e propria sede di concertazione delle politiche per la prevenzione». 
prassi già fornite dall'Agenzia europea per la sicurezza e la salute sul lavoro, istituita con Regolamento del Consiglio dell'Unione europea, al dichiarato fine di promuovere il miglioramento dell'ambiente di lavoro e di fornire agli organi comunitari, agli Stati membri e agli ambienti interessati, le informazioni tecniche, scientifiche ed economiche utili nel campo della sicurezza e della salute sul lavoro ${ }^{39}$.

Le soluzioni di buone prassi, secondo alcuni criteri indicati dall'Agenzia europea, devono dimostrare fasi e metodi da poter adottare all'interno di un luogo di lavoro o all'interno di un'organizzazione, al fine di migliorare le condizioni di vita e di lavoro e/o di ridurre i rischi per la salute e la sicurezza nell'impresa; devono ove possibile concentrarsi sulla prevenzione dei rischi «alla fonte», essere efficaci ed eticamente sostenibili, soddisfare i requisiti legislativi dello Stato membro nel quale sono messe in pratica, contenere sufficienti informazioni da poter essere applicate ad altri luoghi di lavoro dell'Unione europea, nonché contemplare l'attiva partecipazione di tutte le parti coinvolte, in particolare dei lavoratori e dei rappresentanti sui quali incideranno direttamente le azioni intraprese ${ }^{40}$.

In modo non dissimile, le indicazioni della Commissione consultiva permanente tentano di adattare $\mathrm{i}$ suggerimenti trasmessi a livello europeo, richiedendo, anzitutto, dettagliate informazioni sull'impresa che ha adottato le misure di buone prassi e sull'organizzazione che ha proposto il caso concreto, laddove diversa; l'accento è posto, poi, sulla puntuale descrizione dell'attività o situazione che ha determinato l'insorgenza del rischio o l'esigenza di un miglioramento, sulla descrizione della problematica da risolvere e sulla soluzione (tecnica, organizzativa o procedurale), con la precisazione delle misure adottate, della loro attuazione, nonché dei risultati attesi. Rilevano, appunto, i risultati «quantificabili», nonché i vantaggi «non quantificabili», raggiunti o che si attendono, in termini di riduzione degli infortuni e malattie o modalità di lavoro più ergonomiche $\mathrm{o}$ economiche. Non deve mancare, infine, l'indicazione dei costi e de-

39 Cfr. il Regolamento CE n. 2062/94 del Consiglio, del 18 luglio 1994, istitutivo dell'Agenzia europea per la sicurezza e la salute sul luogo di lavoro.

$40 \mathrm{Si}$ veda più esattamente: http://osha.europa.eu/it/practical-solutions/it_ good-practice_2009.pdf, reperibile in archive.beswic.be/it/practical-solutions. 
gli investimenti maturati ${ }^{41}$ e dell'eventuale coinvolgimento dei lavoratori e loro rappresentanti, senza vincoli ai fini della divulgazione. Sempre sulla falsariga delle prescrizioni dell'Agenzia Europea, ulteriore elemento è quello della trasferibilità delle buone prassi in altri luoghi di lavoro, aziende o settori industriali.

Quanto alla richiesta di validazione di buone prassi che riguardino le differenze di genere, la Commissione consultiva permanente ha approvato un apposito documento, fornendo ulteriori indicazioni per la compilazione del modello di presentazione ${ }^{42}$. Con il fine della valorizzazione delle differenze di genere in relazione alla predisposizione delle attività di prevenzione, tale richiesta dovrà essere effettuata attraverso la descrizione di alcuni profili, quali l'inserimento della dimensione di genere all'interno della valutazione dei rischi, la prevenzione dello stress lavoro-correlato in ottica di genere, la programmazione della formazione, informazione e comunicazione in ottica di genere ${ }^{43}$.

Quanto specificamente all'attività di validazione, nulla di più è chiarito né dal legislatore né successivamente dalla Commissione consultiva permanente o in sede ministeriale, facendo presumere la necessaria e forse sufficiente presenza delle, seppur dettagliate, informazioni richieste nel modello di presentazione.

Vero è che l'atto di validazione contribuisce, senza dubbio, a conferire effetti anche dal punto di vista giuridico che, a questo punto, è opportuno prendere in considerazione.

Questa validazione, preceduta, come sottolineato nel decreto, da un'istruttoria tecnica dell'Inail, che provvede ad assicurarne la

41 È la stessa Agenzia europea che mette in luce gli «aspetti economici» della sicurezza, precisando che il miglioramento della salute dei lavoratori rileva anche da una prospettiva economica. Cfr. https://osha.europa.eu/it, ove è inoltre sottolineato che «il costo degli incidenti sul lavoro e delle malattie professionali oscilla tra il 2,6\% e il 3,8\% del PIL»; quindi «una buona gestione della sicurezza in un'azienda è legata al miglioramento delle prestazioni e della redditività».

${ }^{42}$ Il documento è stato approvato nella riunione del 21 settembre 2011: cfr. www.lavoro.gov.it.

43 Cfr. R. Nunin, Lavoro femminile e tutela della salute e della sicurezza: nuovi scenari per una prospettiva di genere dopo il d.lgs. n. 81/2008, in RDSS, 2011, n. 2, p. 399, la quale ha precisato come la «valorizzazione di esperienze e buone prassi gender-sensitive in materia di salute e sicurezza nei luoghi di lavoro andrebbe maggiormente sostenuta». 
più ampia diffusione, consisterebbe, testualmente, in una verifica della validità o convalida delle buone prassi descritte, sulla base di un riscontro di carattere tecnico in relazione alla sussistenza dei requisiti di idoneità richiesti. Dunque, una constatazione e dichiarazione di validità agli effetti legali compiuta da un organo superiore di controllo.

Quali, tuttavia, le conseguenze della conferma e ratifica eseguita dalla Commissione consultiva permanente?

Con questo atto formale di natura amministrativa s'intende, a conclusione di una procedura di verifica avente ad oggetto la completezza ed affidabilità delle prassi stesse, riportarne l'esito e l'attestazione della conformità alla normativa vigente. Più precisamente si tratta, con ogni probabilità, di un vero e proprio «controllo amministrativo», i cui elementi principali, come sottolineato in dottrina, sono ravvisati in un esame della condotta o attività rispetto a diversi criteri, cioè «alle norme, all'opportunità, alla convenienza, all'etica, alla funzionalità», in un giudizio, dall'esito positivo, negativo o misto, ed, infine, nella «misura» adottata dal controllore, che può assumere svariate forme ${ }^{44}$.

Nonostante il silenzio del legislatore e pur in assenza di ulteriori chiarimenti, non si può, in sostanza, escludere il mancato ottenimento dell'atto di validazione, laddove il modello sia manchevole o carente nella descrizione o, addirittura, la buona prassi poco convincente. Certamente il requisito fondamentale al vaglio preventivo dell'Inail e in seguito della Commissione consiste nella presentazione di misure prevenzionistiche realmente e concretamente efficaci.

Indagando oltre nella classificazione dei diversi tipi di controlli amministrativi, si può avvicinare la validazione ad una sorta di «omologazione», ossia un controllo di merito, in sostituzione e a volte in aggiunta alla «approvazione». Con il termine «omologazione» si fa, appunto, riferimento a particolari provvedimenti con i quali è esercitata da parte di determinati organismi, «investiti di un potere di vigilanza e di controllo», un'attività di valutazione e di conferma in merito a situazioni poste in essere anche da soggetti privati ${ }^{45}$. Na-

44 A. Crosetri, voce Controlli amministrativi, in Digesto delle Discipline Pubblicistiche, Torino, Utet, 1989, p. 67 ss.

45 Cfr. A. Palermo, voce Omologazione, in Novissimo Digesto Italiano, XI, Torino, Utet, 1982, p. 905 ss. Una particolare categoria di omologazioni sono le 
turalmente nello svolgimento di tale accertamento è richiesto un elevato grado di specializzazione e preparazione tecnica.

Di certo, da questo momento in poi è possibile distinguere le prassi validate da quelle non validate, in quanto ad esempio precedenti alle previsioni normative del d.lgs. n. 81/2008 o, in ogni caso, non presentate alla Commissione consultiva permanente. Si potrebbe discutere sul beneficio, o meglio, interesse ricavabile dall'impresa che presenti una buona prassi per la validazione, potendolo forse riscontrare nelle strategie ed aspirazioni aziendali ad ottenere forme di «accreditamento», non diversamente da quanto accade in generale nelle prospettive di responsabilità sociale d'impresa ${ }^{46}$. Sicché vi sarebbe un ritorno di immagine e, per così dire, un vantaggio che l'azienda si aspetta. Non si può, forse, nemmeno trascurare un'ambizione delle imprese ad ottenere l'approvazione del proprio comportamento da parte dell'unico organismo deputato per legge ad una verifica.

In ogni caso, una buona prassi validata diventa pubblica, ossia diffusa e trasmissibile in altri contesti e realtà aziendali; acquisisce, insomma, una forma di pubblicità e spendibilità o affidabilità insite nell'atto di convalida. L'elemento di maggiore rilevanza e rispondenza ai principi del diritto dell'Unione europea, conferito dall'atto di validazione è proprio la diffusione e lo scambio delle buone prassi, quali «strumenti di ausilio alle imprese in relazione alla corretta attuazione della normativa in materia di salute e sicurezza sul lavoro» ${ }^{47}$.

L'atto di validazione ha, quindi, una portata dichiarativa esterna, attraverso cui questi strumenti divengono in un certo senso disponibili.

«omologazioni di sicurezza», finalizzate a «rimuovere l'impedimento alla immissione di certi prodotti sul mercato, sul presupposto della verifica del possesso dei requisiti tecnici prescritti in sede normativa per ragioni di sicurezza» (art. 2, d.1. n. 390 del 1982, convertito nella 1. n. 597 del 12 agosto 1982): si veda in proposito A.M. Sandulli, Manuale di diritto amministrativo, Napoli, Jovene, 1989, p. 626 ss., nonché F. TedeschinI, voce Omologazione, in Enc. dir., XXX, Milano, Giuffrè, 1980, p. 90.

46 Sul punto vedi L. Nogler, Gli strumenti di «commercializzazione» del diritto del lavoro: bilancio sociale, certificazione etica e marchio di qualità sociale, in L. Montuschi, P. Tuluini (a cura di), Lavoro e responsabilità sociale dell'impresa, Bologna, Zanichelli, 2006, p. 143 ss.

47 Rilevano, in proposito, le Proposte della Commissione consultiva permanente per una strategia nazionale di prevenzione degli infortuni sul lavoro e delle malattie professionali approvate il 29 maggio 2013. 
Non è forse errato spingersi a sostenere che una buona prassi validata, che suggerisce ad esempio soluzioni tecniche relative ad uno specifico rischio, non può più essere ignorata da altri datori di lavoro nelle medesime circostanze ed alle prese con analoghe problematiche.

Ciò a maggior ragione laddove il d.lgs. n. 81/2008 vi rinvia esplicitamente, ossia richiama le buone prassi in funzione di criterio di adeguatezza dell'adempimento dell'obbligo (cfr. in particolare gli artt. 71, comma 8, e 168, d.los. n. 81/2008). In tali ipotesi, già in precedenza menzionate, le buone prassi validate assumono necessariamente valore vincolante ex lege.

Un esempio particolarmente evidente si rinviene ai sensi dell'art. 181, comma 1, d.lgs. n. 81/2008, che impone al datore di lavoro la valutazione di tutti i rischi derivanti dall'esposizione ad agenti fisici «con particolare riferimento» alle buone prassi, oltreché alle norme di buona tecnica. L'adempimento dell'obbligo sotteso a tale rinvio legale deve intendersi, inoltre, soggetto alla medesima sanzione penale prevista per la mancata valutazione dei rischi in generale dall'art. 28, che vale anche per i Titoli seguenti del decreto n. $81^{48}$. In definitiva, come precisato nell'art. 181, la valutazione dei rischi deve essere effettuata anche attraverso le buone prassi, che assumono in tal caso una valenza obbligatoria ex lege.

5. Contenuto e diffusione delle good practices a livello interno e dell'Unione europea

Occorre ora prendere in considerazione il contenuto delle buone pratiche, promosse e divulgate, in primo luogo, a livello dell'Unione europea.

Gli esempi provengono, essenzialmente, dall'Agenzia europea

48 Cfr. in particolare l'art. 28, comma 3, d.lgs. n. 81/2008, ove è disposto che il contenuto del documento di valutazione dei rischi «deve altresì rispettare le indicazioni previste dalle specifiche norme sulla valutazione dei rischi contenute nei successivi titoli del presente decreto». 
per la sicurezza e la salute sul lavoro, che suggerisce le principali aree interessate ovvero specifica i settori, i tipi di rischio e le categorie di lavoratori coinvolti. L’Agenzia europea svolge, senza dubbio, un ruolo molto importante in materia ormai da diversi anni, organizzando, tra l'altro, in cooperazione con gli Stati membri, la «Settimana europea per la sicurezza e la salute sul lavoro», rectius le «Campagne ambienti di lavoro sani e sicuri», nell'ambito delle quali rileva il «Premio» per le buone pratiche, che consiste in un riconoscimento alle organizzazioni distintesi per i metodi innovativi di promozione della sicurezza sui luoghi di lavoro ${ }^{49}$.

A titolo meramente esemplificativo, tra le recenti good practices premiate e pubblicate dall'Agenzia europea vi sono quelle promosse dai Paesi Bassi, con oggetto l'accrescimento della consapevolezza, tra i conducenti di carrelli elevatori, delle misure prevenzionistiche e dalla Spagna, relative ad un modello di prevenzione dei rischi per le piccole imprese. Tra le prassi encomiate vi sono quelle dell'Austria, volte ad incoraggiare i lavoratori nel segnalare i problemi di sicurezza e della Francia, mirate ad integrare la salute e la sicurezza nella strategia aziendale ${ }^{50}$.

Nei suoi documenti l'Agenzia europea si è soffermata sul ruolo complementare delle iniziative volontaristiche rispetto allo strumento legislativo, mettendone in risalto il valore aggiunto. Con riferimento, in particolare, alla «Healthy Workplaces Campaign», nell'ambito della quale è annualmente ripetuta la «European Good Practice Awards Competition», si promuove «the inclusion of occupational safety and health management in organisations' corporate social responsibility policies», a fondamento di una «sustainable risk prevention culture in Europe» ${ }^{51}$.

49 Il «Premio» per le buone pratiche 2014-2015, ad esempio, si propone di mettere in luce esempi significativi relativi alla gestione dello stress e dei rischi psicosociali sul lavoro; nell'ambito della campagna «Ambienti di lavoro sani e sicuri ad ogni età 2016-2017», il «Premio» per le buone pratiche mira, invece, «a mettere in evidenza esempi significativi di organizzazioni che gestiscono attivamente la sicurezza e la salute sul lavoro nel contesto di una forza lavoro che invecchia».

50 https://osha.europa.eu/it/competitions/good-practice-award_2012-2013.

51 Healthy Workplaces. Working together for risk prevention. European Good Practice Awards 2012-2013, European Agency for Safety and Health at Work, 2013: www.osha.europa.eu. 
V'è da chiarire che l'obiettivo perseguito a livello dell'Unione europea, secondo le richieste dell'Agenzia europea, come già anticipato, è quello di applicare, con gli opportuni accorgimenti, i modelli di buone pratiche risultati efficaci in un determinato luogo di lavoro anche in ambienti differenti. Tuttavia, posto che queste prassi devono, anzitutto, soddisfare i requisiti legislativi dello Stato membro in cui vengono attuate, è prevedibile che le relative misure ed informazioni non siano immediatamente trasferibili da uno Stato all'altro; si tratta, infatti, di contesti giuridici, per lo più, molto diversi tra di loro e lo stesso concetto di buona prassi prevenzionistica è suscettibile di molteplici e diversi significati.

Si può, fin d'ora premettere che all'ampia diffusione in Europa delle migliori pratiche non corrisponde un'adeguata definizione, nonché disciplina, comparabile con quella italiana, che rispetto ad altri ordinamenti può, quantomeno, vantare una normativa novellata recentemente.

All'interno del nostro ordinamento, come anticipato, le pratiche aziendali volontarie sono state congegnate ed applicate fin da prima della loro effettiva regolamentazione, al fine di incrementare ed adattare alle diverse realtà organizzative il complesso apparato di norme in tema di tutela della sicurezza sul lavoro. Pur nella varietà delle manifestazioni, esse possono sostanzialmente essere ricondotte a interventi e procedure tali da fornire un reale contributo nella soluzione di specifici problemi in determinate aziende, settori produttivi, aree di rischio. In sostanza, «azioni» e «metodologie che possano risultare virtuose ai fini del miglioramento degli standard di sicurezza sui luoghi di lavoro» ${ }^{52}$.

È, dunque, riscontrabile la tendenza, soprattutto nelle realtà di grandi dimensioni, a ideare soluzioni tarate sui rischi esistenti e dirette a migliorare la salute e la sicurezza dei lavoratori, pur senza il proposito di una loro validazione e conseguente pubblicità.

In linea generale, si rinvengono misure volte a implementare la formazione dei prestatori di lavoro, ad esempio attraverso la conse-

52 G. Natullo, Riassetto normativo e sistema delle fonti, in G. NAtullo, R. SAntucci (a cura di), Ambiente e sicurezza sul lavoro. Quali tutele in vista del Testo Unico?, Milano, Franco Angeli, 2008, p. 29. 
gna di opuscoli relativi al sistema di prevenzione aziendale, in modo che possa essere controllato dagli stessi creditori di sicurezza. L'attenzione è prestata, inoltre, alla capillare ed accurata fornitura dei dispositivi di protezione individuale in diversi punti di distribuzione degli stabilimenti aziendali. Di frequente, v'è poi l'indizione di riunioni, che coinvolgono i principali soggetti della sicurezza, ossia il datore di lavoro o un suo rappresentante, il responsabile del servizio di prevenzione e protezione, il medico competente ed il rappresentante dei lavoratori per la sicurezza, con la finalità di discutere le problematiche in materia con assiduità ed in anticipo rispetto alla riunione periodica di cui all'art. 35, d.lgs. n. 81/2008 ${ }^{53}$. È preso in esame anche il rischio legato alla trasferta del lavoratore con la predisposizione di accorgimenti utili finalizzati ad evitare infortuni e malattie.

Non mancano appositi progetti riguardanti la verifica e l'analisi periodica del comportamento dei lavoratori durante il lavoro, al fine di far emergere eventuali scorrettezze e valorizzare viceversa gli atteggiamenti positivi; dai lavoratori stessi, in seguito ricompensati con riconoscimenti o premi, sono, a volte, raccolte proposte e suggerimenti di miglioramento dell'ambiente di lavoro ${ }^{54}$.

Assumono rilevanza, infine, le giornate dedicate alla sicurezza sul lavoro, interamente finalizzate a promuovere la cultura della sicurezza, nel corso delle quali sono previste «sessioni di educazione, formazione e condivisione di procedure e messaggi chiave», alternate a prove pratiche e a simulazioni ${ }^{55}$.

53 Deve essere menzionato il progetto «Sviluppo Imprese in Sicurezza», che Confindustria, in collaborazione con Inail, Federmanager e Fondirigenti, ha realizzato con l'obiettivo di favorire una maggiore diffusione della cultura della prevenzione dei rischi lavorativi, «non solo come imperativo etico e di responsabilità sociale, ma come fattore determinante per la qualità del lavoro e la competitività dell'impresa». L'iniziativa, sviluppata nel quadriennio 2009-2012, è stata promossa e sostenuta dal Comitato Tecnico Sicurezza di Confindustria e ha coinvolto oltre 6.000 imprenditori; nell'ambito del progetto sono state raccolte le buone prassi aziendali in tema di sicurezza sul lavoro, proposte dai rappresentanti delle oltre 70 aziende testimonial: www.confindustria.it.

54 Tale approccio è generalmente indicato con BBS, ossia Behaviour Based Safety.

55 Si tratta, ad esempio, della c.d. «Safety Week», evento promosso da Luxottica Group S.p.a. nel corso della settimana dedicata alla sicurezza (10-14 marzo 
Accanto a queste devono essere menzionate le prassi raccolte e diffuse dagli enti pubblici incaricati dallo stesso d.lgs. n. 81/2008 e, in particolare, dall'Inail, nonché quelle «validate» dalla Commissione consultiva permanente: soluzioni organizzative, procedurali o tecniche, «coerenti con la normativa vigente».

Occorre segnalare, anzitutto, soluzioni procedurali che introducono nuovi processi di valutazione dei rischi, ad esempio con la partecipazione attiva ed il coinvolgimento diretto dei lavoratori stessi nella valutazione e gestione dei rischi presenti nelle aree lavorative e nelle rispettive postazioni di lavoro ${ }^{56}$; nonché soluzioni che forniscono strumenti di supporto per l'applicazione della procedura standardizzata per la valutazione dei rischi ai sensi dell'art. 29, comma 5, d.lgs. n. 81/2008 ${ }^{57}$. Con particolare riferimento a quest'ultima buona prassi va rimarcata la sua «idoneità» a «individuare i pericoli presenti in azienda», «identificare le misure di prevenzione e protezione»e «definire il programma di miglioramento» ${ }^{58}$, in tal modo completando ed arricchendo le previsioni legislative.

In ordine allo specifico e complesso ambiente lavorativo della sanità v'è da citare la buona prassi procedurale che si sofferma sulle differenze di genere quali fattori che possono incidere sul rischio professionale, con l'obiettivo di realizzare il miglioramento continuo dell'ambiente di lavoro, delle attrezzature e dell'organizzazione, rispetto alle caratteristiche dei due sessi ${ }^{59}$.

2014), che ha coinvolto oltre 7500 persone dei 7 stabilimenti produttivi italiani in un programma di cinque giorni di iniziative ed attività dedicate alla prevenzione, all'educazione e alla formazione sul tema della sicurezza: www. luxottica.com; si veda, altresì, l'iniziativa «Safety day» promossa da Eni S.p.a.

$56 \mathrm{Si}$ veda la buona prassi presentata da Tarkett S.p.a., validata dalla Commissione consultiva permanente il 25 settembre 2013, volta ad aumentare la partecipazione dei lavoratori nel procedimento di valutazione dei rischi.

57 Cfr. la buona prassi «Impresa sicura», presentata dall'Associazione Impresa Sicura, validata dalla Commissione consultiva permanente il 27 novembre 2013.

58 Così è espressamente descritta la citata buona prassi «Impresa sicura».

59 Vedi la buona prassi «Sicurezza nel prendersi cura [...] in ottica di genere», presentata da U.O.C. Prevenzione, Protezione e Sicurezza sui luoghi di lavoro, Fondazione Policlinico Tor Vergata-Roma, validata dalla Commissione consultiva permanente il 29 maggio 2013, che prende in esame la problematica dell'applicazione degli strumenti prevenzionistici nell'ottica di genere in ambito sanitario, in considerazione della natura eccezionalmente articolata delle attività lavorative e dei rischi a cui sono esposti i lavoratori e le lavoratrici, dell'innovazione e del cambiamento 
Meritano attenzione, poi, soluzioni di tipo organizzativo, come quella incentrata sul «miglioramento dei comportamenti individuali di sicurezza dei lavoratori» e sulla comunicazione tra i soggetti coinvolti nell'ambiente di lavoro dei cantieri (committente, direzione lavori, impresa appaltatrice, imprese subappaltatrici, preposti, lavoratori), ove coesistono diverse aree di rischio ${ }^{60}$.

Si possono ricordare, in ambito sanitario, anche soluzioni di tipo sia organizzativo sia procedurale che consentono una nuova strategia in ordine alla movimentazione manuale dei pazienti, con riguardo alla scelta delle misure di prevenzione da adottare basate sull'addestramento e sull'utilizzo degli ausili meccanici, considerati strumento essenziale per l'eliminazione del rischio alla fonte ${ }^{61}$.

continuo di tecnologie, materiali, prodotti chimici e farmaceutici, dell'esposizione pressoché generale delle professioni sanitarie a rischi di tipo biologico. Da questo punto di vista, le differenze di genere che interessano la valutazione di qualsiasi rischio lavorativo sono raggruppate in «differenze biologiche» e «differenze socioculturali» ed anche le misure di prevenzione e protezione sono riferite e scelte in base alle differenze di genere.

60 Vedi la buona prassi «Sicuri per mestiere: una storia non ordinaria di sicurezza in cantiere», presentata dalla Cooperativa Muratori e Braccianti di Carpi, validata dalla Commissione consultiva permanente il 27 novembre 2013. Sono stati coinvolti circa 2000 lavoratori di circa 35 imprese subappaltatrici e appartenenti a oltre 30 diverse nazionalità. La soluzione ha previsto, in particolare, incontri periodici del «Gruppo di progetto», composto dallo staff di cantiere (capo commessa, direttore di cantiere, capo cantiere, responsabili e addetti alla sicurezza), dal responsabile dello sviluppo delle risorse umane e dal responsabile del servizio di prevenzione e protezione, al fine dell'esame dei dati, dell'individuazione delle criticità, della messa a punto di misure correttive; ha previsto, inoltre, incontri periodici con le imprese subappaltatrici e i loro preposti, incontri periodici con i lavoratori, per la comunicazione dell'avanzamento dell'intervento, dei suoi risultati, delle eventuali misure correttive delle criticità riscontrate, nonché premiazioni periodiche dei lavoratori delle imprese più virtuose dal punto di vista dei comportamenti di sicurezza.

${ }_{61} \mathrm{~V}$. la buona prassi «Movimentazione centrata sulla persona», presentata dall'Associazione IGIEA, che, a partire dal 1998, ha appunto sviluppato il modello definito «movimentazione centrata sul malato», volto a promuovere l'autonomia del malato come intervento preventivo specifico nell'eliminazione o riduzione dei rischi. La soluzione, validata dalla Commissione consultiva permanente il 27 novembre 2013, ha realizzato un intervento assistenziale personalizzato capace di trasformare l'oggetto della movimentazione in soggetto attivo, gestore della tutela della propria salute. Si tratta, in sostanza, di una nuova interpretazione della movimentazione dei pazienti, non più centrata sull'operatore ma sulla persona. La sperimentazione ha coinvolto circa 1200 pazienti, 250 operatori sanitari, per un totale di circa 6000 osservazioni. 
Inoltre, soluzioni di tipo tecnico sono quelle, ad esempio, indirizzate a dotare i cantieri edili, anche se differenti per dimensioni e tipologie di lavoro, di sistemi di rilevazione in tempo reale per la valutazione dei rischi ${ }^{62}$.

Tecnica ed organizzativa insieme è, per concludere la casistica, la prassi validata che intende «sviluppare una cultura in materia»e portare, come espressamente precisato, «la salute e la sicurezza nella vita di tutti i giorni», sensibilizzando i lavoratori, accrescendo in loro la percezione dei pericoli e riducendo i comportamenti pericolosi, attraverso la predisposizione di un percorso formativo ad hoc ${ }^{63}$.

Accanto alle soluzioni indicate ve ne sono altre identificate come mere «linee operative» per l'organizzazione aziendale, riferite ad esempio alla pulizia ed al mantenimento dello stato di efficienza degli indumenti di protezione individuale; esattamente linee guida rivolte alle aziende «al fine della corretta somministrazione e manutenzione dei dispositivi di protezione individuale» ${ }^{64}$. Dunque, prassi per lo più chiarificatrici ed esplicative della disciplina legale.

Le menzionate buone prassi validate dalla Commissione consultiva permanente seguono, sostanzialmente, lo schema del citato modello di presentazione. Ciò che ha rilevanza pare essere l'impatto positivo dell'utilizzo delle buone prassi sul sistema di prevenzione,

62 Si tratta della buona prassi «Sistemi di rilevazione in tempo reale per la valutazione dei rischi nei cantieri edili», validata dalla Commissione consultiva permanente il 17 aprile 2013, sperimentata presso quattro cantieri edili della Regione Campania e di cui è stata data informazione dalla Consulenza Tecnica Accertamento Rischi e Prevenzione (CONTARP)-INAIL Direzione Regionale Campania. La buona prassi utilizza nuove tecnologie per realizzare il controllo, il monitoraggio e la valutazione della sicurezza nei cantieri edili. Il sistema, basato su hardware (varchi elettronici, sensori, centraline riceventi, elaboratori elettronici) e software/database, ha consentito l'identificazione istante per istante dei vari operatori che accedono all'area di cantiere, il controllo della loro posizione in relazione ai possibili rischi, il corretto utilizzo dei DPI, suggerendo correttivi in tempo utile per intervenire prima che si manifestino situazioni di rischio.

63 Cfr. la buona prassi «La sicurezza non è un gioco», presentata da Hera S.p.a. e validata dalla Commissione consultiva permanente il 27 novembre 2013.

64 Cfr. la buona prassi presentata da Assosistema-Confindustria, validata dalla Commissione consultiva permanente il 30 maggio 2012, con la quale sono fornite alle imprese le informazioni di base per una corretta manutenzione dei dispositivi di protezione individuale. 
dovendo essere documentata la riduzione (raggiunta o attesa) degli infortuni e delle malattie professionali, dunque dei costi sociali.

Si tratta, in definitiva, di misure prevenzionistiche che, variamente, integrano o completano le previsioni legislative, in alcuni casi proprio arricchendole, in altri limitandosi invece ad esplicarne e specificarne i contenuti o a suggerirne le modalità corrette di applicazione.

Vero è che il d.lgs. n. 81/2008 ha disciplinato solo le buone prassi validate (art. 2, comma 1, lett. v) ma, come chiarito, il panorama è più vasto e si compone di molteplici esperienze pratiche, che possono prescindere da una loro validazione.

Non è allora propriamente possibile un approccio unitario al tema, prospettandosi diverse ipotesi interpretative.

\section{Inquadramento e qualificazione giuridica}

Esaurita la casistica, è, a questo punto, necessario interrogarsi in merito alla qualificazione giuridica delle buone prassi con riguardo a quelle non validate, ossia ancora in via di sperimentazione o comunque non sottoposte al giudizio della Commissione consultiva permanente che ne abbia constatato l'efficacia e ne abbia promosso la diffusione e trasferibilità. Si può fin d'ora anticipare, infatti, che le buone prassi validate e rese pubbliche, proprio in ragione di tali caratteristiche, possono essere, in un certo senso, considerate già parte dell'obbligo di sicurezza di cui all'art. 2087 c.c. e, dunque, necessarie (cfr. il paragrafo 7).

Pur nel variegato quadro delle possibili prassi prevenzionistiche, è opportuno ora soffermarsi sulle soluzioni semplicemente introdotte dal datore di lavoro che ne fa uso e sulle conseguenze che ne derivano.

In generale, non è azzardato accostare tali buone prassi agli usi aziendali, unanimemente considerati quali spontanei comportamenti assunti dal datore di lavoro nei confronti dei propri dipendenti, a loro favorevoli e reiterati nel tempo, che acquistano il carattere della obbligatorietà e vincolatività. Nonostante la molteplicità delle rico- 
struzioni giurisprudenziali in materia, la tendenza è, infatti, quella ad approdare all'unitario risultato della obbligatorietà del comportamento reiterato del datore di lavoro; l'erogazione di benefici supplettivi, in virtù del ripetersi nel tempo, diviene oggetto di un obbli$\mathrm{go}^{65}$. La reiterazione della condotta favorevole, cui deve affiancarsi il requisito della spontaneità e quindi della mancanza di un obbligo nell'adozione del comportamento, è, infatti, «condizione necessaria e sufficiente» per la trasformazione della «originaria liberalità in una prestazione dovuta ${ }^{66}$. Il datore di lavoro è, quindi, sia "protagonista» di comportamenti liberamente adottati, sia «produttore» di un vincolo giuridico nei confronti dei destinatari ${ }^{67}$.

Indagando più in generale la stessa nozione di «prassi», è evidente come la dottrina, anche straniera, la riconduca da tempo agli usi, invero distinti tra usi normativi e contrattuali; pratiques, ovvero, usages, quali «insieme di comportamenti giuridicamente rilevanti $»^{68}$. Prassi, come «modo d'agire» 0 «attività volontarie dirette a realizzare risultati concreti positivi ${ }^{69}$.

Pare particolarmente appropriato il confronto con gli usi aziendali, la cui natura giuridica è, tuttavia, ancora molto dibattuta e controversa, sia in dottrina che in giurisprudenza. V'è, inoltre, la necessità di riflettere sulla collegata ed incerta questione del mantenimento dei trattamenti di maggior favore eventualmente praticati nei confronti dei prestatori di lavoro. Il vero nodo interpretativo attiene sia alla costruzione dal punto di vista giuridico dell'istituto sia alla

65 Cfr. P. Ichino, Il contratto di lavoro, I, Milano, Giuffrè, 2000, p. 252 ss.

66 Cfr., in proposito, S. Liebman, Prassi aziendale e regolazione dei rapporti di lavoro nell'impresa: fonte consuetudinaria o prodotto dell'autonomia collettiva?, in $A D L, 2009$, n. 3, p. 704 ss.

67 L. Spagnuolo Vigorita, Usi aziendali e diritto del lavoro, in MGL, 1989, p. 684 ss.

68 Così J. Ghestin, Rapport de synthèse, in Le rôle de la pratique dans la formation du droit, Travaux de l'Association H. Capitant, Tome XXXIV, 1983, p. 3 ss. Sulla nozione di «prassi» cfr. G. Alpa, voce Prassi, in Digesto delle Discipline Privatistiche, Sezione Civile, XIV, Torino, Utet, 1996, p. 142; si veda, altresì, P. Perlingieri, Prassi, principio di legalità e scuole civilistiche, in ID., Scuole, tendenze e metodi. Problemi di diritto civile, Napoli, Edizioni Scientifiche Italiane, 1989, p. 226 ss.

${ }^{69}$ Cfr. J.L. Sourioux, Rapport Francais, in Le rôle de la pratique dans la formation du droit, cit., p. 85. 
possibile revocabilità dell'atto di gestione del datore di lavoro/imprenditore $^{70}$.

Occorre, in primo luogo, tenere nettamente distinti gli usi aziendali dagli usi normativi, cioè dalle consuetudini quali fonti del diritto, collocate per ultime all'art. 1 delle Disposizioni sulla legge in generale, il cui «ruolo nell'ambito lavoristico è praticamente inesistente» ${ }^{71}$.

Le consuetudini rappresentano un modo tipico di produzione del diritto, rectius, come precisato, «il modo spontaneo, naturale, incosciente, informale», contrapposto, viceversa, a quello «riflesso, artificiale, cosciente, formale» della legge ${ }^{72}$. Gli usi normativi, di cui all'art. 8 delle preleggi, che hanno efficacia per le materie regolate dalla legge solo in quanto espressamente richiamati, ovvero secundum legem, sono, inoltre, ex art. 1374 c.c., fonti di integrazione del regolamento contrattuale, insieme alla volontà delle parti, alla legge, all'equità ${ }^{73}$.

Per completezza, v'è da richiamare in proposito un'altra norma che, ugualmente, secondo la comune opinione, si riferisce agli usi in senso normativo, non potendo essere, invece, riferita agli usi aziendali. Si tratta dell'art. 2078 c.c., inserito tra le disposizioni del codice civile dedicate al lavoro e relativo all'efficacia degli usi nel rapporto di lavoro, i quali si applicano in mancanza di disposizioni di legge (e di contratto collettivo), ossia praeter legem, e prevalgono su di esse, se più favorevoli per i lavoratori ${ }^{74}$. «L'operatività» degli usi

70 Si veda in particolare L. Spagnuolo Vigorita, Gli usi aziendali, Morano, Napoli, 1965, p. 57 ss.; nonché ID., voce Usi aziendali, in Enc. dir., Aggiornamento, Milano, Giuffrè, 2001, p. 1120.

71 L. Montuschi, Il sistema generale delle fonti giuslavoristiche, in M. PersiaNI (a cura di), Le fonti del diritto del lavoro, cit., p. 414.

72 N. Bobbio, voce Consuetudine (teoria generale), in Enc. dir., XI, Milano, Giuffrè, 1961, p. 426 ss.

73 Cfr. F. Galgano, Il negozio giuridico, in Trattato di diritto civile e commerciale, già diretto da A. Cicu, F. Messineo, L. Mengoni, continuato da P. Schlesinger, Milano, Giuffrè, 2002, p. 58 ss.; nonché A. AsouinI, Usi legali e usi negoziali, in RDComm, 1944, I, p. 72 e ID., Integrazione del contratto con le "clausole d'uso», in Scritti giuridici, III, Padova, Cedam, 1961, p. 376.

74 Cfr. F. Santoro Passarelli, Nozioni di diritto del lavoro, Napoli, Jovene, 1961, p. 16. Per un commento al riguardo cfr. P. Bellocchi, Art. 2078 c.c., in G. Amoroso, V. Di Cerbo, A. Maresca, Diritto del lavoro, vol. I, Milano, Giuffré, 2017, p. 742 ss. 
normativi, come fonte integrativa degli effetti del contratto ai sensi dell'art. 1374 c.c., è, allora, specificata in ordine al rapporto di lavoro dall'art. 2078 c.c., «nel senso di consentire la prevalenza sulle norme legali dispositive di quelle consuetudini che comportano un trattamento più favorevole al prestatore di lavoro» ${ }^{75}$.

Quanto specificamente agli usi aziendali, al contrario diffusi ed utilizzati, le ricostruzioni dottrinali e giurisprudenziali, come anticipato, sono tra loro alquanto differenti e ancora lontane da soluzioni univoche e consolidate.

Secondo la tesi tradizionalmente sostenuta dalla giurisprudenza, l'uso aziendale è qualificato come uso negoziale, suscettibile, $e x$ art. 1340 c.c., di inserzione, come clausola d'uso, nel contratto individuale di lavoro, con idoneità a derogare soltanto in melius la disciplina collettiva ${ }^{76}$. Per la formazione degli usi aziendali, riconducibili alla categoria degli usi negoziali, è necessaria «la sussistenza di una prassi generalizzata, che si realizza attraverso la mera reiterazione di comportamenti posti in essere spontaneamente e non già in esecuzione di un obbligo» nei confronti dei dipendenti anche di una sola azienda e che comporti per essi un trattamento di miglior favore rispetto a quello previsto dalla legge o dalla contrattazione collettiva; come precisato, tali condizioni migliorative non possono essere derogate in peius dalla contrattazione collettiva, posto che gli usi «si inseriscono nei singoli contratti individuali e non già nei contratti collettivi nazionali o aziendali, e che l'esclusione di tale inserzione può avvenire soltanto in base alla concorde volontà delle parti» ${ }^{77}$.

75 E. Del Prato, voce Usi nel rapporto di lavoro, in Enc. Giur. Treccani, XXXII, Roma, 1994, p. 1 ss.

76 Cfr., in particolare, Cass., S.U., 30 marzo 1994, n. 3134, in ADL, 1995, I, p. 374, con nota di S. Liebman, Gli "usi aziendali" davanti alle Sezioni Unite e in GC, 1995, p. 231 ss., con nota di G. Mammone, Principio volontaristico e formazione dell'uso aziendale; Cass., S.U., 17 marzo 1995, n. 3101, in MGL, 1995, p. 162, con nota di L. MASINI, Gli usi aziendali: incertezze giurisprudenziali e prospettive collettive. Alcune più recenti sentenze continuano ad aderire a questo indirizzo tradizionale: v., ad esempio, Cass. 5 settembre 2014, n. 18780, in RGL, 2015, II, p. 317 ss., con nota di E.M. TERENZIO, Usi aziendali e contratto individuale di lavoro: un ritorno al passato?; Cass. 13 dicembre 2012, n. 22927, in RIDL, 2013, II, p. 725, con nota di G. QUADRI, Brevi considerazioni sulla natura dell'uso aziendale e sulla possibilità di modifica ad opera del contratto collettivo.

77 V. Cass. 13 dicembre 2012, n. 22927, cit. 
Dunque, l'inserimento diretto ed automatico nel contratto individuale di lavoro può essere escluso solo con la volontà di datore di lavoro e lavoratore.

La dottrina civilistica si è ampiamente soffermata sull'esatto significato da attribuire alle clausole d'uso. Rispetto ai già menzionati usi legali o normativi, contraddistinti dalla convinzione della doverosità dei comportamenti assunti, la c.d. opinio iuris ac necessitatis, quelli negoziali sono stati considerati dall'orientamento maggioritario quali «pratiche generali» ovvero «clausole proprie di determinati mercati», osservate dalle parti «come norme negoziali dettate dalle circostanze del mercato»; «clausole di determinati rami del commercio, di determinate categorie professionali $\rangle^{78}$. Secondo un altro indirizzo, alle clausole d'uso corrispondono le clausole solitamente inserite nei contratti ${ }^{79}$.

In ogni caso, la riconduzione degli usi aziendali alle clausole d'uso non è stata esente da critiche. Più esattamente, si è escluso che possa essere richiamato l'art. 1340 c.c. «per sostenere l'efficacia autonoma del c.d. uso aziendale in tema di prestazioni supplettive del datore di lavoro» ${ }^{80}$. Quanto alle conseguenze di tale indirizzo interpretativo, rimarrebbe, essenzialmente, insoluto il problema dell'estensione del comportamento datoriale, dunque del vincolo giuridi-

78 Per un approfondimento sulla distinzione tra usi legali e negoziali cfr. A. AsouINI, Usi legali e usi negoziali, cit., p. 74, secondo cui «quando la pratica crea gli usi dei mercati tipici di certe merci, generalizzando le clausole contrattuali che corrispondono alle particolari esigenze tecniche di tali mercati, non considerate dalla legge, crea precisamente usi negoziali»; ID., Integrazione del contratto con le clausole d'uso, cit., p. 381. Si veda, inoltre, R. Scognamiglio, Dei contratti in generale. Artt. 1321-1352, in Commentario del codice civile a cura di Antonio Scialoja e Giuseppe Branca, Bologna-Roma, Zanichelli-Il Foro italiano, 1970, p. 236, che chiarisce come l'espressione «clausole di uso» implica, «sia dal punto di vista storico che per il suo significato logico, una pratica diffusa in un certo mercato». Sul punto v., anche, S. Rodotì, Le fonti di integrazione del contratto, Milano, Giuffrè, 2004, p. 71.

79 F. Messineo, voce Contratto (dir. priv.), in Enc. dir., Milano, Giuffrè, 1961, IX, p. 939; M. Bianca, Diritto civile, Milano, Giuffrè, 2002, p. 73 ss. Per una ricostruzione delle varie tesi v. L. VAlente, Consuetudini e usi nel rapporto di lavoro subordinato, Milano, Giuffrè, 2006, p. 64.

80 Cfr. in senso critico, ex plurimis, L. Spagnuolo Vigorita, Gli usi azienda$l i$, cit., p. 190 ss., il quale considera il ricorso all'art. 1340 c.c. privo di «giuridica consistenza». 
co ad esso sotteso, anche nei confronti dei lavoratori assunti successivamente e non diretti beneficiari ${ }^{81}$.

È così emerso in dottrina un differente orientamento, peraltro accolto anche dalla giurisprudenza, che ha considerato l'uso o la prassi aziendale come espressione dell'autonomia collettiva. Precisamente, l'obbligatorietà del comportamento datoriale, oggetto dell'uso aziendale, è stata ricondotta alla (finzione della) stipulazione di un accordo collettivo non scritto ${ }^{82}$. Di modo che, al fine di «tutelare giuridicamente l'aspettativa che il beneficio concesso ripetutamente a singoli lavoratori possa aver determinato nella generalità dei dipendenti circa un loro futuro diritto a prestazioni analoghe» è parso necessario «dare per acquisita la possibilità di ricostruire i profili di una valenza collettiva del comportamento unilaterale dell'imprenditore» ${ }^{83}$. Dal punto di vista degli effetti, l'uso, collocato nella dimensione collettiva, può essere modificato anche in peius ad opera di un contratto collettivo sopravvenuto.

Nella varietà e complessità delle ricostruzioni, alcune sentenze hanno prestato attenzione ai rapporti collettivi all'interno dell'azienda, sul presupposto che l'uso sia fonte di «un obbligo unilaterale di carattere collettivo, che agisce sul piano dei singoli rapporti individuali allo stesso modo e con la stessa efficacia di un contratto collettivo aziendale» ${ }^{84}$.

81 L. Spagnuolo Vigorita, Gli usi aziendali, cit., p. 184; nonché P. LamberTUCCI, Usi aziendali e rapporto di lavoro: brevi note, in DML, 2009, p. 435, secondo il quale «appare del tutto fuorviante poggiare sull'art. 1340 c.c. la collocazione sistematica dell'uso aziendale».

82 L. Spagnuolo Vigorita, Usi aziendali e diritto del lavoro, cit., p. 685 ss.

83 S. Liebman, Individuale e collettivo nel contratto di lavoro, Milano, Giuffrè, 1993, p. 26 ss.

84 Cass. 6 novembre 1996, n. 9690, in RIDL, 1997, II, p. 692, con nota di A. Occhino, Usi aziendali e gratuità delle attribuzioni patrimoniali, in GC, 1997, p. 1347 ss., con nota di S. Liebman, "Usi aziendali”, volontà negoziale dell'imprenditore e autonomia collettiva, e in OGL, 1997, I, p. 5 ss., con nota di L. CASTELVETRI, L'uso aziendale come manifestazione di autonomia collettiva dell'imprenditore verso i lavoratori; Cass. 27 novembre 1999, n. 13294, in MGL, 2000, p. 584, con nota di S. Liebman, Comportamenti unilaterali dell'imprenditore, usi aziendali e teoria delle fonti di regolamentazione del rapporto di lavoro; nonché già Cass. 9 agosto 1991 n. 8705, in GCM, 1991; Cass. 19 marzo 1986 n. 1916, in GC, 1986, I, 1906, ove è precisato che gli effetti giuridici vincolanti della prassi aziendale sono riferibili al rapporto collettivo, «nel quale i lavoratori vengono considerati, non sin- 
Le perplessità sorte in ordine a quest'ultima tesi hanno riguardato prevalentemente la necessità di individuare un interlocutore sindacale, dalla cui effettiva presenza, insieme al «complessivo comportamento delle parti», dipende la possibilità di «collocare ragionevolmente il fenomeno sul piano dei rapporti collettivosindacali» ${ }^{85}$.

Sulla scia di questo indirizzo, altre decisioni della Cassazione hanno incluso gli usi aziendali tra le non meglio precisate «fonti sociali eteronome del rapporto di lavoro», insieme ai contratti collettivi ed al regolamento d'azienda ${ }^{86}$. La formula, peraltro alquanto «ambigua» ${ }^{87}$, allude a fonti che «non costituiscono espressione di funzione pubblica, ma neppure realizzano meri interessi individuali, in quanto dirette a conseguire un'uniforme disciplina dei rapporti con riferimento alla collettività impersonale dei lavoratori di

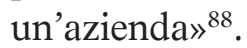

Anche in questo caso non sono mancate critiche accese in me-

golarmente, ma unitariamente, in relazione ai loro bisogni comuni». Tra le sentenze più recenti cfr. Cass. 11 luglio 2007, n. 15489, in DeJure; Cass. 10 luglio 2008, n. 18991, in GCM, 2008; Cass. 11 marzo 2010, n. 5882, in $L G, 2010$, p. 785, con nota di E. BAVASSO, Sostituzione automatica della contrattazione collettiva del cessionario e usi aziendali; Cass. 7 aprile 2010, n. 8240, in DeJure. In dottrina v., in particolare, P. LAMBERTUCCI, Usi aziendali e rapporto di lavoro: brevi note, cit., p. 442 , per il quale «l'uso aziendale viene ad operare come una fonte di regolamentazione dei rapporti di lavoro», al pari del contratto collettivo aziendale; ID., Gli usi aziendali tra contratto individuale e assetti collettivi dell'impresa: il "bilancio giurisprudenziale”, in LG, 2014, p. 827 ss.; nonché F. LuNARDON, Il contratto collettivo e le altre fonti (legge, usi, regolamenti), in Il sistema delle fonti nel diritto del lavoro, Atti delle giornate di studio di diritto del lavoro Foggia-Baia delle Zagare, 25-26 maggio 2001, Milano, Giuffrè, 2002, p. 106 ss.

85 Si vedano le osservazioni di S. Liebman, Prassi aziendale e autonomia privata, in $M G L, 1999$, p. 45. In merito ai «rilevanti elementi di distinzione tra uso e contratto collettivo aziendale» cfr. A. LASSANDARI, Il contratto collettivo aziendale e decentrato, Milano, Giuffrè, 2001, p. 159.

86 Cass., S.U., 13 dicembre 2007, n. 26107, in RIDL, 2008, II, p. 539 ss., con nota di G. QuAdri, Usi aziendali, "fonti sociali" e autonomia collettiva; Cass. 27 maggio 2008, n. 13816, in $A D L, 2009$, p. 749 e in RCDL, 2008, p. 966; Cass. 8 aprile 2010, n. 8342, in NGL, 2010, p. 682; Cass. 2 settembre 2013, n. 20085, in DeJure; Cass. 23 marzo 2016, n. 5768, in DeJure; nonché già Cass. 17 febbraio 2000, n. 1773, in RIDL, 2000, II, p. 598, con nota di L. CAstelvetri, Ma l'uso aziendale esiste?

87 L. Montuschi, Il sistema generale delle fonti giuslavoristiche, cit., p. 414.

88 Cass. 17 febbraio 2000, n. 1773, cit. 
rito all'utilizzo di una categoria, quale quella delle «fonti sociali», di «incerta collocazione nel sistema delle fonti di regolamentazione del rapporto di lavoro» ${ }^{89}$ e che «risulta un ibrido, radicalmente extra ordinem $\gg^{90}$. Nel tentativo di definizione degli usi aziendali, le espressioni utilizzate dalla Corte di Cassazione sono, insomma, risultate confuse, non offrendo il contributo necessario per dirimere il nodo qualificatorio ed individuare il fondamento della vincolatività giuridica.

Più di recente in dottrina è stata valorizzata la condotta unilaterale del datore di lavoro, quale «manifestazione di volontà a rilevanza collettiva», finalizzata «ad assumere nuove obbligazioni ed a modificare quelle esistenti in senso favorevole» ${ }^{91}$. La natura degli usi aziendali è quella di atti negoziali unilaterali, «espressione dell'autonomia collettiva dell'imprenditore», senza la necessità di previsione di un accordo collettivo tacito. Tale ricostruzione, spostando nuovamente l'attenzione sul piano collettivo e superando quello dei singoli rapporti individuali, ha attribuito valore vincolante al comportamento reiterato del datore di lavoro nei confronti della collettività e generalità dei prestatori di lavoro. Gli usi aziendali sono, così, ricompresi tra le «fonti aziendali» ed assimilabili al contratto collettivo aziendale ed al regolamento interno d'impresa e, quali «fonti eteronome» di integrazione della disciplina del rapporto di lavoro, risultano eliminabili o modificabili da successivi contratti collettivi ${ }^{92}$.

Si può, in definitiva, sostenere come convivano differenti e, per certi versi, contrastanti indirizzi, tesi a valorizzare la dimensione individuale o, viceversa, quella collettiva dell'uso, pur pervenendo tutti, salvo rare eccezioni, all'identico e pressoché assodato risultato della vincolatività della condotta datoriale favorevole.

89 S. Liebman, Comportamenti unilaterali dell'imprenditore, usi aziendali e teoria delle fonti di regolamentazione del rapporto di lavoro, cit., p. 596.

90 L. Castelvetri, Ma l'uso aziendale esiste?, cit., p. 612 ss.

91 G. QuAdri, Usi aziendali e autonomia negoziale. Regole consuetudinarie e modificazioni unilaterali nel rapporto di lavoro, Napoli, Edizioni Scientifiche Italiane, 2008, p. 317 ss.

92 G. QUADRI, Usi aziendali e autonomia negoziale. Regole consuetudinarie e modificazioni unilaterali nel rapporto di lavoro, cit., p. 346 ss. 


\subsection{Le buone prassi quali atti unilaterali integrativi del contratto di lavoro}

A seguito di questa breve riflessione che mette in luce l'incertezza interpretativa esistente intorno alla problematica, tuttora di grande attualità, della configurazione giuridica degli usi aziendali, è opportuno indagare meglio lo speciale ruolo rivestito dalle buone prassi prese in esame e trarre qualche conclusione.

Si può, anzitutto, escludere che tali buone prassi siano assimilabili alle menzionate clausole d'uso nella loro accezione considerata nel paragrafo precedente. È, infatti, evidente come le soluzioni progettate, per lo più, in riferimento a singoli e specifici rischi all'interno dell'azienda, per quanto trasferibili a situazioni analoghe, difficilmente si concilino con le clausole «normalmente convenute nella pratica corrente» ${ }^{93}$, né, tantomeno, con le «clausole di stile formate da usi generali del mercato» ai sensi dell'art. 1340 c.c. ${ }^{94}$. Ciò vale soprattutto in considerazione dei mutevoli parametri dell'evoluzione della tecnica e dell'esperienza che connotano l'adempimento dell'obbligo di sicurezza.

Volendo, allora, attualizzare e fare propria un'espressione sostenuta in passato, si può ritenere che anche le menzionate buone prassi utilizzate in materia di sicurezza sul lavoro, quali comportamenti endoaziendali, siano in realtà «prassi osservate da singoli imprenditori, cioè usi individuali» ${ }^{95}$.

Quale, però, il corretto riferimento normativo cui ancorare l'obbligatorietà della prassi?

Premesso che la questione qualificatoria degli strumenti in esame è certamente complessa ed è necessaria molta prudenza nel richiamare le norme civilistiche ${ }^{96}$, sembra opportuno valorizzare la

93 Secondo l'interpretazione di M. BIANCA, Diritto civile, cit., p. 73.

94 Cfr. L. Mengoni, Diritto civile, in L. Mengoni, A. Proto Pisani, A. Orsi Battaglini, L'influenza del diritto del lavoro su diritto civile, diritto processuale civile, diritto amministrativo, in DLRI, 1990, p. 16 ss. In giurisprudenza si veda in particolare Trib. Milano 10 luglio 1998, in MGL, 1999, p. 41 ss., con nota di S. Liebman, Prassi aziendale e autonomia privata, cit.

95 L. Mengoni, Diritto civile, cit., p. 16.

96 V. al riguardo le osservazioni di S. Liebman, Prassi aziendale e autonomia privata, cit., p. 44, che mette in luce «la scarsa coerenza interna degli orientamenti giurisprudenziali in materia». 
dimensione individuale della prassi aziendale. Più esattamente, l'atto unilaterale del datore di lavoro deve essere esaminato nell'ottica di un comportamento concludente cui corrisponde l'accettazione (tacita) da parte dei lavoratori destinatari. Di conseguenza, ai sensi dell'art. 1333 c.c., si può ritenere che la condotta volontaria determini una modifica o integrazione del contenuto del contratto individuale da cui sorge un diritto in capo ai beneficiari ${ }^{97}$.

La prassi, per sua natura rivolta ad una pluralità di persone, manterrebbe una rilevanza interindividuale, non necessariamente collettiva, integrando i singoli contratti dei lavoratori e generando in loro un diritto soggettivo a percepire quel trattamento di favore, riversato nel contratto individuale di lavoro ${ }^{98}$.

Tale implementazione avviene nell'ambito contrattuale e il comportamento concludente del datore di lavoro configura una «proposta diretta a modificare il contratto», assumendo valore il «silenzioso accoglimento della pratica» da parte dei lavoratori direttamente coinvolti ${ }^{99}$. Lo schema utilizzabile è quello della proposta diretta a concludere un contratto da cui derivino obbligazioni solo per il proponente ex art. 1333 c.c. Si può, così, sostenere che la reiterazione del comportamento unilaterale del datore di lavoro dia vita ad una vera pretesa giuridica in capo ai lavoratori destinatari, poiché si ve-

97 Per un approfondimento in merito alla disciplina di cui all'art. 1333 c.c., seppur con riguardo alla conclusione di un nuovo contratto tra le parti e non alla modifica di un contratto a prestazioni corrispettive già esistente v. R. SACCO, Il contratto, in Trattato di diritto civile italiano, diretto da F. Vassalli, Torino, Utet, 1975, p. 31 ss.; ID., La conclusione dell'accordo, in E. GABRIELLI (a cura di), I contratti in generale, Torino, Utet, 2006, p. 79 ss.; cfr., altresì, G. BenEDETTI, Dal contratto al negozio unilaterale, Milano, Giuffrè, 1969, p. 121 ss.

98 Sulla nozione di interessi collettivi si veda F. SANTORo Passarelli, voce $\mathrm{Au}$ tonomia collettiva, in Enc. dir., Milano, Giuffré, 1959, p. 369 ss., che definisce l'interesse collettivo come interesse «di quelle collettività di persone ad un bene idoneo a soddisfare un bisogno comune», ossia interesse «superiore all'interesse degli individui appartenenti al gruppo»; nonché ID., Nozioni di diritto del lavoro, Napoli, Jovene, 1961, p. 21 ss. Sul tema cfr., inoltre, M. Persiani, Saggio sull'autonomia privata collettiva, Padova, Cedam, 1972, p. 22 ss. Con specifico riferimento alla tutela della sicurezza dei lavoratori v. G. SupPIEJ, Il diritto dei lavoratori alla salubrità dell'ambiente di lavoro, in RIDL, 1988, p. 442 ss.

99 Questa è la tesi già sostenuta in merito agli usi aziendali da L. GaLANTINO, La prassi aziendale, in $D L, 1968$, I, p. 222 ss. In tema di comportamento concludente si veda C. Smuraglia, Il comportamento concludente nel rapporto di lavoro, Milano, Giuffrè, 1963, p. 19 ss. 
rifica un'integrazione del contenuto del contratto individuale di lavoro attraverso una sorta di loro tacita accettazione ${ }^{100}$.

Ciò sicuramente vale, secondo le regole di carattere generale dei contratti, nei confronti di quei prestatori di lavoro che hanno goduto del trattamento migliorativo, verso i quali cioè il comportamento può considerarsi effettivamente concludente ${ }^{101}$. Quid iuris per coloro che sono entrati in contatto con il datore di lavoro in un secondo momento perché assunti successivamente?

Il punto cruciale sul quale le diverse ricostruzioni in tema di usi aziendali si sono finora soffermate, mostrando qualche segno di cedimento o di debolezza è, come anticipato, quello della ricomprensione tra i fruitori delle misure ulteriormente corrisposte, di quei lavoratori inizialmente non coinvolti, perché assenti, dunque estranei al sorgere del vincolo giuridico.

La richiamata visione collettiva aziendale ha, tuttavia, consentito di attribuire efficacia vincolante verso la generalità dei lavoratori, non limitata cioè ai soli beneficiari dell'attribuzione, «bensì nei confronti di tutti i lavoratori rientranti, anche in futuro, nei presupposti individuati dal comportamento datoriale» ${ }^{102}$.

Diversamente, con riferimento alla tesi che prospetta lo schema di cui all'art. 1333 c.c., è stato sottolineato che «valore concludente» non può essere attribuito «ad un contegno che non sia stato posto in essere in direzione di chi si pretende destinatario della proposta» ${ }^{103}$. Ecco perché, nei confronti dei lavoratori assunti successivamente, si rende quantomeno necessario ricorrere ad un criterio interpretativo

100 Sul tema, in particolare, cfr. L. BALESTRA, Introduzione al diritto dei contratti, Il Mulino, Bologna, 2015, p. 115, secondo cui «nell'ipotesi del contratto con obbligazioni del solo proponente, la sfera del destinatario della proposta è suscettibile di essere incisa soltanto se quest'ultimo non reagisca rifiutando».

101 Cfr., in particolare, le osservazioni critiche in tal senso di Cass. 17 febbraio 2000 , n. 1773, cit.

102 P. Lambertucci, Usi aziendali e rapporto di lavoro: brevi note, cit., p. 436 SS.

103 L. Mengoni, In tema di usi aziendali, in $M G L, 1978$, p. 472 ss.; cfr., tuttavia, L. Galantino, La prassi aziendale, cit., p. 230, che ha precisato come, rivolgendosi il datore di lavoro a lavoratori qualificati unicamente dalla funzione che esplicano, «usufruiranno delle prestazioni supplettive non solo i lavoratori cui queste ultime sono direttamente riferite, ma anche coloro che nella funzione subentreranno». 
che legga, ai sensi dell'art. 1362, comma 2, c.c., il comportamento del datore di lavoro per ricavarne la sua volontà ${ }^{104}$.

Anche per quanto specificamente concerne l'individuazione dei lavoratori beneficiati dalle best practices congegnate ed applicate all'interno dell'azienda, valgono i principi testé richiamati, almeno astrattamente. Vale a dire che le pratiche che si instaurano nei rapporti tra determinati contraenti rilevano al fine dell'interpretazione del contratto come comportamento complessivo delle parti, ex art. 1362, comma 2, c.c.

È allora condivisibile l'indirizzo che valorizza la ricerca della volontà del datore di lavoro. Sebbene tale criterio non abbia avuto molta fortuna nelle interpretazioni giurisprudenziali relative alla qualificazione giuridica degli usi aziendali, perché considerato, in un certo senso, superfluo rispetto a quello della reiterazione nel tempo della condotta datoriale, è opportuno, a questo punto, fare ad esso riferimento ${ }^{105}$.

In concreto, però, si potrebbe forse ipotizzare che un problema interpretativo di questo genere non si ponga.

Sulla scorta della giurisprudenza che in materia di usi aziendali ha richiamato «la fattispecie astratta dell'art. 1333 c.c.»e la necessaria «ricerca dell'intento del datore di lavoro di obbligarsi (unilateralmente) attraverso il suo comportamento» ${ }^{106}$, non si può prescindere dall'indagine sulla volontà di adottare misure migliorative anche per

104 L. Mengoni, In tema di usi aziendali, cit., p. 471, secondo il quale «nei confronti dei lavoratori assunti successivamente anche un solo atto di estensione ad essi del beneficio fornisce di regola un elemento sufficiente di interpretazione del contratto nel senso di un adeguamento originario dell'intenzione comune dei contraenti alla prassi già in vigore nell'impresa (art. 1362, co. 2, c.c.)».

105 Nell'ordinamento francese l'usage è considerato una «pratica generale che si avvale di una certa ancienneté», più precisamente una pratica ripetuta e costante, che risulta dalla volontà del datore di lavoro e, in ragione della sua persistenza, può far nascere nell'animo dei lavoratori una legittima convinzione o aspettativa in merito al suo mantenimento: v. E. Peskine, C. Wolmark, Droit du travail, Paris, Dalloz, 2016, p. 62, ove si chiarisce, inoltre, che il regime degli usagses d'entreprise presenta forti analogie con quello degli engagements unilatéraux, quali impegni unilaterali nati semplicemente dall'affermazione della volontà del datore di lavoro di accordare un determinato vantaggio.

106 Trib. Milano 10 luglio 1998, cit., che ha precisato come tale ricerca «deve essere particolarmente rigorosa» proprio in ragione dell'impegno assunto da una sola delle parti e «per effetto di una condotta riferibile esclusivamente alla stessa». 
il futuro. Detta indagine, condotta «sulla concludenza del comportamento, al fine di determinarne il significato» ${ }^{107}$, nel caso di specie non può che far emergere l'univoca volontà datoriale anche nei confronti dei lavoratori non assunti.

Le buone prassi prevenzionistiche sono, infatti, di per sé dirette ad una molteplicità di lavoratori identificabili orientativamente per le mansioni cui sono addetti e per i relativi rischi cui sono sottoposti; tutto ciò porta ad escludere che esse siano rivolte solo ad alcuni e non a tutti coloro che si vengono a trovare nella condizione oggettiva presa in considerazione. Vero è, allora, che le buone prassi generalmente utilizzate, proprio per la finalità che sottendono, non possono considerarsi ad personam, non essendo corrisposte in ragione di requisiti soggettivi propri di ciascun dipendente.

Le prassi aziendali in esame, insomma, non sono suscettibili di un'applicazione frazionata, dovendo piuttosto ritenersi applicabili o a tutti i lavoratori che si trovano (e si troveranno) nella medesima situazione di rischio o a nessuno.

Sulla base del «principio volontaristico» ${ }^{108}$ si può spiegare il sorgere del vincolo giuridico a favore della generalità dei dipendenti, attribuendo rilevanza all'intento di modificare in senso migliorativo il trattamento dei dipendenti anche per il futuro. Accanto alla volontarietà dei comportamenti ossia alla spontaneità, quantomeno iniziale, coincidente con la «convinzione nell'autore che le elargizioni concesse ai lavoratori non sono dovute» ${ }^{109}$, assume importanza in alcune pronunce il consapevole impegno di obbligarsi (definitivamente). La vincolatività dell'uso non può, pertanto, prescindere dall'apprezzamento dell'effettiva «volontà di vincolarsi da parte del datore di lavoro» ${ }^{110}$.

107 In proposito v. C. SmuRaglia, Il comportamento concludente nel rapporto di lavoro, cit., p. 101 ss., il quale chiarisce gli «indici» della concludenza, tra cui rileva la continuità e abitualità della corresponsione.

108 Cfr., al riguardo, P. Tullini, Uso aziendale e principio volontaristico, in RIDL, 1991, II, p. 806 ss.

109 L. MontusCHI, Il sistema generale delle fonti giuslavoristiche, cit., p. 415.

110 V., in particolare, Pret. Ancona, 2 novembre 1990, in RIDL, 1991, II, p. 806, con nota di P. TulLINI, Uso aziendale e principio volontaristico, cit.; al riguardo v., inoltre, Cass. 27 novembre 1999, n. 13294, cit.; nonché Cass. 11 luglio 2007, n. 15489 , cit., ove è precisato che «l'uso aziendale presuppone non già una sem- 
In definitiva sotto questo profilo, si può sottolineare che le prassi aziendali sono di per sé intraprese con l'esatto obiettivo di innalzare i livelli di sicurezza all'interno dell'ambiente di lavoro e di ridurre ulteriormente i rischi, offrendo ai prestatori di lavoro strumenti aggiuntivi e nello stesso tempo necessari. Se tutto ciò è vero, non si può allora negare la stessa tutela rafforzata anche nei confronti di chi subentrerà nelle medesime situazioni di rischio, al di là degli originari destinatari.

Oltre alla questione della obbligatorietà con riguardo ai soggetti vincolati, ossia alla c.d. ultrattività del comportamento, v'è anche da chiarire il problema della modificabilità o revocabilità dello stesso ad opera del datore di lavoro.

Tirando le fila del ragionamento fin qui condotto, si può ritenere che le buone prassi non possano essere dismesse unilateralmente, proprio perché vincolanti, rectius riconducibili all'obbligo di sicurezza ai sensi dell'art. 2087 c.c. Invero, come fin da subito è stato sottolineato, «l'opzione volontaria si perde (o rischia di perdersi) quando entra in contatto con l'area del dovere di sicurezza» ${ }^{111}$.

È ipotizzabile che raggiunto così un più alto livello di sicurezza, cioè "la massima possibile", come formulato dalla costante giurisprudenza di legittimità, non è dato discrezionalmente scendere.

Ci si potrebbe, tuttavia, chiedere se possano costituire delle eccezioni quelle situazioni nelle quali il datore di lavoro può venirsi a trovare, come i cambiamenti della struttura organizzativa dell'azienda o la sopravvenuta incompatibilità delle prassi aziendali con le esigenze economiche dell'impresa ${ }^{112}$. Il mantenimento dei trattamenti

plice reiterazione di comportamenti, ma uno specifico intento negoziale di regolare anche per il futuro determinati aspetti del rapporto lavorativo».

111 L. Montuschi, Dai principi al sistema della sicurezza sul lavoro, in ID. (dir.), La nuova sicurezza sul lavoro, cit., p. 3 ss., che ha sottolineato come «le buone prassi e i comportamenti etici esauriscono il loro ruolo creativo nel momento in cui la volontarietà si stempera e cede all'irresistibile vis attractiva esercitata dal vincolo giuridico obbligatorio, il quale decolora l'originaria matrice volontaria di tanto accrescendo l'ambito dei comportamenti doverosi».

112 In proposito si veda, ad esempio, Cass. 19 marzo 1986, n. 1916, cit., ove è stato chiarito che «la volontà negoziale non è revocabile in senso unilaterale»; «non ha, quindi, rilevanza eliminativa la decisione aziendale di abbandonare la prassi precedente per la sopravvenuta incompatibilità di essa con le esigenze economiche dell'impresa». 
di maggior favore costituisce davvero sempre un vincolo ineliminabile per l'imprenditore?

Non si può in effetti escludere, come del resto è già stato affermato in alcuni sporadici interventi dei giudici, che impedimenti di carattere organizzativo facciano venir meno alcuni vantaggi precedentemente accordati ai lavoratori. Si pensi, ad esempio, all'eliminazione del diritto a fruire di pause giornaliere nei confronti di lavoratori addetti all'utilizzo di particolari macchinari, a causa dell'oggettivo cambiamento della situazione regolata, ossia dell'introduzione ed installazione di diversi macchinari, cui consegue una postura meno scomoda ed un minor tempo di applicazione del personale; premesso che, a fronte di un diritto acquisito per uso aziendale, il datore di lavoro non potrebbe incidere unilateralmente, la Cassazione ha sostenuto, infatti, che «l'uso presuppone la identità della situazione regolata, mentre nel caso in esame si è in presenza di una nuova organizzazione del lavoro, implicante l'utilizzazione di macchinari diversi, con tempi di applicazione diversi» ${ }^{113}$.

Si pensi, poi, alla possibilità che il datore di lavoro decida di non protrarre l'utilizzo di una determinata misura aggiuntiva divenuta non più efficace ed incisiva rispetto all'obiettivo perseguito. Oltre a non essere più necessaria dal punto di vista organizzativo, una buona prassi potrebbe, in effetti, non rispondere più alle caratteristiche di efficacia richieste. La buona prassi potrebbe pertanto essere dismessa ovvero sostituita con altro e diverso accorgimento, in quanto appunto considerata non più rispondente ai risultati attesi in termini di riduzione degli infortuni e malattie. Sul presupposto dell'effettività della tutela, alcune misure potrebbero, dunque, essere abbandonate, senza pregiudizio per i lavoratori. Del resto, alla base della volontà datoriale di impegnarsi nell'adozione di buone prassi v'è il requisito della loro efficacia; perciò la verifica in ordine alla sua mancanza potrebbe giustificare il venir meno del vincolo.

Questa possibilità di revoca unilaterale delle condizioni lavorative può essere condivisa solo laddove concessa sulla base di circo-

113 Cfr. Cass. 21 agosto 2009, n. 18593, in Foro it., 2010, I, c. 98. 
stanze definibili appunto oggettive e non, invece, se assunta del tutto arbitrariamente ${ }^{114}$.

Ci si potrebbe, invero, chiedere se si tratti di un atto di revoca in senso stretto e non piuttosto del mero accertamento da parte del datore di lavoro del venir meno della situazione di fatto su cui la volontà si è formata, ossia del cambiamento del contesto organizzativo dal quale non si può prescindere.

Un'ultima considerazione attiene al ruolo che, in definitiva, possono assumere le buone prassi, così accostate agli usi. Lo spunto per una tale riflessione deriva anche dalle valutazioni formulate dalla dottrina straniera in ordine all'efficacia delle clausole commerciali, destinate ad anticipare l'intervento del legislatore ${ }^{115}$.

Con riferimento alle buone prassi, quanto meno a quelle che successivamente siano state validate e che abbiano ottenuto una rilevanza pubblica ed un'ampia diffusione, si può finanche sostenere che costituiscano una fonte di ispirazione e un'occasione di progettazione legislativa.

In particolare nella materia della tutela della sicurezza nei luoghi di lavoro, si potrebbe ritenere che la tendenza sarà quella a vagliare e collaudare buone prassi, le quali possono con ogni probabilità essere, addirittura, trasposte nella legislazione, quindi imposte esplicitamente tramite lo strumento normativo.

Ecco, allora, che le conseguenze di vincolatività e obbligatorietà già insite in una buona prassi utilizzata in azienda, per di più con la

114 Si veda L. Spagnuolo Vigorita, Usi aziendali e diritto del lavoro, cit., p. 685 ss., secondo il quale «il modificarsi delle circostanze alle quali il comportamento datoriale si è oggettivamente riferito esclude la permanenza in vita del vincolo». La giurisprudenza di legittimità, seppur in rare occasioni, ha ritenuto eliminabile unilateralmente l'uso aziendale da parte del datore di lavoro in considerazione del «trascorrere del tempo», sufficiente a differenziare il trattamento riservato ai lavoratori: cfr. Cass. 12 agosto 2009, n. 18263, in GCM, 2009, p. 1259, che, nonostante l'applicabilità del trattamento di miglior favore nei confronti dei lavoratori assunti in epoca successiva alla formazione dell'uso aziendale, ha affermato anche la facoltà dell'imprenditore di escludere i nuovi lavoratori dal beneficio.

115 B. Goldman, Rapport général, in Le rôle de la pratique dans la formation $d u$ droit, cit., p. 164 ss., secondo cui «la pratica contribuisce alla formazione del diritto», nonché J. Ghestin, Rapport de synthèse, ivi, cit., p. 5. Cfr., inoltre, le considerazioni di G. Alpa, voce Prassi, cit., p. 140, il quale si riferisce in particolare all'esperienza del contratto di conto corrente, all'apertura di credito, al deposito in cassette di sicurezza. 
convinzione di raggiungere il preciso fine di elevare i livelli di sicurezza, nonché successivamente validata, sono probabilmente destinate ad essere prima o poi formalmente accolte dal legislatore.

\section{Volontarietà e necessità}

Le buone prassi calate all'interno del rapporto di lavoro, soddisfano le esigenze di sicurezza ai sensi dell'art. 2087 c.c. Infatti, pur trattandosi di soluzioni in prevalenza migliorative rispetto a quanto specificamente disposto nel d.lgs. n. 81/2008, si può ritenere che rappresentino le «misure necessarie» che l'imprenditore è tenuto ad adottare al fine della garanzia della salute nell'ambiente di lavoro, in ottemperanza ai mutevoli parametri della particolarità del lavoro, dell'esperienza e della tecnica. Si verifica, propriamente, una integrazione dell'area dell'obbligo, posto che l'art. 2087 c.c. attraverso le buone prassi si dilata.

In proposito, non si deve dimenticare che il dato normativo rimarca in più punti la volontarietà dell'iniziativa datoriale, rectius dell'adozione di tali soluzioni, anche relativamente all'esercizio del potere di disposizione da parte degli organi di vigilanza (cfr. artt. 2, comma 1, lett. v e 302-bis, d.lgs. n. 81/2008).

Proprio con riferimento alle prassi validate, ai sensi della definizione legislativa, si può, tuttavia, tratteggiare un dovere (implicito) di adozione, in quanto soluzioni evidentemente rese pubbliche e quindi disponibili sul mercato. Di conseguenza non possono essere ignorate da parte del datore di lavoro diligente le misure di sicurezza così individuate e ancor prima le esigenze di sicurezza che le hanno fatte sorgere. Non è cioè trascurabile l'esistenza di prassi validate, destinate a scongiurare una determinata situazione di rischio lavorativo.

L'esigenza di sicurezza deve, comunque, trovare una risposta da parte del datore di lavoro, libero semmai nell'individuazione della prassi più consona al problema manifestatosi. La volontarietà va quindi coniugata con la necessità di una scelta di soluzione; una scelta, dunque, "necessitata" riguardante la singola misura, che si pone in alternativa ad un'altra. 
Si tratta, allora, di mezzi a disposizione per raggiungere il fine della massima sicurezza in azienda.

È importante ricordare, al riguardo, il parere del Consiglio di Stato del 4 aprile 2005 sullo schema di decreto legislativo per il riassetto delle disposizioni vigenti in materia di sicurezza e tutela della salute dei lavoratori, di attuazione della legge delega n. 229/2003 $3^{116}$. Ivi era stata affermata la necessità di assicurare che «in sede interpretativa e applicativa» l'osservanza delle buone prassi, nonché delle norme di buona tecnica, fosse intesa come «una delle forme di attuazione dell'art. 2087 del codice civile, senza esaurirne la portata precettiva». Lo schema di decreto censurato dal Consiglio di Stato aveva, infatti, disposto che l'osservanza delle buone prassi «costituisce attuazione» dell'art. 2087 c.c. (art. 1, comma 4), inducendo a ritenere che il contenuto della norma codicistica fosse alquanto ridotto con un «evidente abbassamento del livello di tutela».

La possibilità di una scelta in capo al datore di lavoro pur nel perseguimento del dovere di protezione della salute è, in un certo senso, supportata attraverso la comparazione tra le buone prassi e gli Approved Codes of Practices regolati nell'Health and Safety at Work Act del Regno Unito, che, per quanto non del tutto assimilabili, sono individuati quali metodi utilizzati al fine del rispetto delle previsioni di legge; metodi o «suggerimenti» specifici, non obbligatori, considerati però «sufficienti per conformarsi alla legge» relativamente al particolare profilo preso in esame, eppur sostituibili con misure alternative deputate al medesimo scopo ${ }^{117}$ (cfr. sul punto il paragrafo 8.2.).

116 In proposito cfr. M. Ricci, Ambiente di lavoro e responsabilità sociale d'impresa, in L. Montuschi, P. TuLLINI (a cura di), Lavoro e responsabilità sociale dell'impresa, cit., p. 55.

117 Esattamente è precisato che «if you follow the advice you will be doing enough to comply with the law in respect of those specific matters on which the Code gives advice»: www.hse.gov.uk/legislation/legal-status.htm. Tale indicazione rimarca la differenza rispetto alle buone prassi semplicemente definite nel d.lgs. $n$. $81 / 2008$ come «adottate volontariamente». In generale in merito ai Codes of practice cfr. le riflessioni di M. Rusciano, A proposito del «Libro Bianco sul mercato del lavoro in Italia», in www.unicz.it/lavoro/lavoro.htm, 2002; nonché di F. BANo, Diritto del lavoro e nuove tecniche di regolazione: il soft law, in LD, 2003, n. 1, p. 49 ss. 
Più complesso ed articolato è naturalmente il discorso con riferimento alle buone prassi non validate, in verità non contemplate nel d.lgs. n. 81/2008 ma ampiamente diffuse, ossia a quelle misure la cui esistenza e, soprattutto, efficacia non è stata certificata ${ }^{118}$. La riflessione a maggior ragione deve riferirsi anche alle soluzioni non sottoposte al vaglio della Commissione consultiva permanente. Se non si può escludere che tali soluzioni vengano prese come riferimento per misurare la diligenza esigibile in capo al datore di lavoro, va, però, rimarcata la mancata divulgazione e l'assenza di una loro verifica che ne certifichi in concreto l'utilità e la convenienza.

Del resto, le misure di sicurezza più avanzate in base all'esperienza ed al progresso tecnico sono da considerarsi esigibili ove possiedano «un margine» di effettiva «sperimentazione» e «conoscenza» ${ }^{119}$; ove cioè siano già acquisizioni tecniche e scientifiche ${ }^{120}$.

118 Vero è che la validazione ha finora riguardato un numero circoscritto di prassi, rinvenibili solo negli anni 2012 e 2013.

119 In merito al complesso legame tra il dovere di sicurezza e le acquisizioni scientifiche si vedano le considerazioni formulate da C. Smuraglia, Sicurezza del lavoro e obblighi comunitari. I ritardi dell'Italia nell'adempimento e le vie per uscir$n e$, in $R I D L, 2002$, p. 190. La giurisprudenza ha da tempo affermato che «l'art. 2087 c.c. non può essere dilatato fino a comprendervi ogni ipotesi di danno, sull'assunto che comunque il rischio non si sarebbe verificato in presenza di ulteriori accorgimenti di valido contrasto, perché in tal modo si perverrebbe all'abnorme applicazione di un principio di responsabilità oggettiva»: in tal senso v., ad es., Cass., sez. lav., 17 maggio 2013, n. 12089, in DeJure; Cass., sez. lav., 5 dicembre 2001, n. 15350, in RIDL, 2002, p. 778; Cass., sez. lav., 3 settembre 1997, n. 8422, in $G C$, 1998, I, p. 79. Si veda, inoltre, Cass., sez. lav., 10 giugno 2016, n. 11981, in www. olympus.uniurb.it, secondo cui la diligenza del datore di lavoro si traduce nel «dovere di conoscere quei saperi e di adottare quelle tecniche considerate più attendibili nell'ottica di perseguire il fine indicato dall'art. 2087».

120 In proposito è nota la pronuncia della Corte costituzionale 25 luglio 1996, n. 312, in RIDL, 1997, II, p. 15, cit., ove è stata, invece, precisata la doverosità delle «misure che, nei diversi settori e nelle differenti lavorazioni, corrispondono ad applicazioni tecnologiche generalmente praticate», risultando censurata solo la «deviazione dei comportamenti dell'imprenditore dagli standard di sicurezza propri, in concreto e al momento, delle diverse attività produttive». Sul punto v., in particolare, C. Zoli, Sicurezza del lavoro e certezza del diritto, Intervento, in A. Vallebona (a cura di), Colloqui Giuridici sul Lavoro, Milano, 2009, p. 55, per il quale «non ci si deve limitare agli standard generalmente praticati, se non sono sufficienti a garantire il più alto livello di sicurezza, sempre che tali tecnologie siano, ovviamente, disponibili, cioè quantomeno sperimentate e diffuse nel mercato». Si deve ricordare, altresì, la sentenza della Corte di Giustizia, sez. III, 14 giugno 2007, causa n. C-127/05, in RIDL, 2008, II, p. 3, con nota di O. Bonard, La Corte di Giustizia 
Mentre il datore di lavoro deve conformare il proprio comportamento rispetto alle cautele già disponibili sulla base del progresso scientifico, non pare, invece, possa considerarsi tenuto ad attivarsi, promuovendo o sperimentando ex novo i più evoluti sistemi prevenzionistici, ancora privi di un appurato fondamento. Sicché, come già sostenuto in passato con riferimento ai tre «elementi essenziali» che connotano l'art. 2087 c.c., «non si potrà pretendere che il datore di lavoro introduca mezzi di prevenzione del tutto nuovi e non ancora sperimentati», ma avrà invece l'obbligo di adottarli non appena saranno «acquisiti dall'esperienza e dalla tecnica» ${ }^{121}$. Una valutazione quindi particolarmente delicata, da condursi caso per caso, che richiama l'ampia e complessa questione del legame tra diritto e sapere scientifico e tecnologico.

Si deve anche sottolineare che le prassi adottate (validate e non) sono destinate ad essere inserite nel documento di valutazione dei rischi, come si può evincere dall'art. 28 , comma 2, d.lgs. n. 81 , che ne indica il contenuto, riferendosi alle «misure di prevenzione e di protezione attuate» e ai «dispositivi di protezione individuali adottati» (lett. b) e, soprattutto, al «programma delle misure ritenute opportune per garantire il miglioramento nel tempo dei livelli di sicurezza» (lett. c) ${ }^{122}$; ciò conferisce alle buone prassi il crisma della

e l'obbligo di sicurezza del datore di lavoro: il criterio del reasonably practicable è assolto per insufficienza di prove, con la quale si è escluso che il Regno Unito, nel circoscrivere nei limiti di quanto «ragionevolmente praticabile» l'obbligo di sicurezza del datore di lavoro, sia venuto meno agli obblighi che gli incombono in virtù dell'art. 5, nn. 1 e 4, della direttiva 89/391.

121 C. Smuraglia, La sicurezza del lavoro e la sua tutela penale, Milano, Giuffrè, 1974, p. 85; nonché ID., Nuovi fattori di rischio, prevenzione e responsabilità del datore di lavoro. Quali regole?, in RGL, 2005, I, p. 14, il quale ha precisato che «se conoscenze diffuse rivelano che la scienza e la tecnica hanno individuato un rischio e hanno indicato le misure idonee a prevenirlo, allora non ci sono giustificazioni: quelle misure debbono essere adottate, e se non lo si fa ognuno si assume le proprie responsabilità».

122 Si ricordi che ai sensi dell'art. 15, comma 1, lett. t, d.lgs. n. 81/2008, tra le misure generali di tutela rientra esattamente «la programmazione delle misure ritenute opportune per garantire il miglioramento nel tempo dei livelli di sicurezza», anche «attraverso l'adozione di buone prassi». In ordine al rapporto tra misure generali di tutela, di cui all'art. 15, d.lgs. n. 81, e art. 2087 c.c. cfr. le riflessioni di G. Bolego, Art. 15, in C. Zoli (a cura di), Principi comuni, in L. Montuschi (dir.), La nuova sicurezza sul lavoro, cit., p. 146, secondo il quale le categorie indicate nel 
obbligatorietà, elevandole, in tal modo, al rango di misure doverose di sicurezza.

Ne consegue una concezione ampia e moderna dell'obbligo di sicurezza, quale rinvenibile nel d.lgs. n. 81, essendo diverse le opzioni offerte al fine di garantire più elevati standard di sicurezza; è, così, senza dubbio «esaltato il profilo prevenzionale» ${ }^{123}$.

Ci si potrebbe, diversamente, domandare se le buone prassi ambiscano ad andare oltre lo stesso disposto dell'art. 2087 c.c.; sono, cioè, ipotizzabili prassi ulteriori rispetto alle «misure necessarie» richiamate nella norma codicistica?

In verità, l'ampiezza e la non definitiva delimitazione del contenuto dell'obbligo sembra suggerire la non percorribilità di una simile interpretazione, essendo arduo immaginare di poter superare una norma dal perimetro elastico, la cui «vitalità» ed «originaria vis innovativa ${ }^{124}$ non è stata scalfita nel tempo. A ciò si aggiunga che la stessa definizione di «salute» contenuta nell'art. 2, comma 1, lett. o, d.lgs. n. 81, è molto ampia, ricomprendendo lo «stato di completo benessere, fisico, mentale e sociale, non consistente solo in un'assenza di malattia o d'infermità».

In astratto, prassi realmente ulteriori rispetto all'art. 2087 c.c. potrebbero essere forse solo quelle, invero difficilmente immaginabili, attuate in mancanza di un rischio concreto o di una precisa esigenza di sicurezza all'interno dell'ambiente di lavoro; prassi, cioè, apparentemente superflue, slegate dall'adempimento doveroso dell'obbligo di sicurezza e, pertanto, del tutto facoltative.

primo comma dell'art. 15 «devono intendersi come specificazione del generale obbligo di sicurezza».

123 P. Albi, La sicurezza sul lavoro e la cultura giuridica italiana tra vecchio e nuovo diritto, cit., p. 84. Cfr., altresì, L. MenghinI, L'evoluzione degli strumenti giuridici volti a favorire l'effettività della prevenzione, in DSL, 2017, n. 2, p. 25, secondo cui il sistema delineato nel d.lgs. n. 81/2008 «porta a compimento l'evoluzione per la quale la prevenzione effettiva degli infortuni e delle malattie, più che su norme precettive variamente sanzionate o su disposizioni tecnico-produttive, deve far leva su una programmazione della prevenzione che si basi su analisi, ricerche, percorsi procedurali, scelte organizzative, codici di condotta e di buone prassi».

124 L. Montuschi, Dai principi al sistema della sicurezza sul lavoro, in ID. (dir.), La nuova sicurezza sul lavoro, cit., p. 9. 
7.1. Quale incidenza sui profili di responsabilità? Alcune ipotesi possibili

Per comprendere meglio l'incidenza degli strumenti presi in esame rispetto al generale dovere di sicurezza, l'attenzione deve essere, altresì, prestata al profilo dell'accertamento della responsabilità datoriale.

Quanto, in particolare, all'assolvimento dell'onere probatorio l'adozione di soluzioni organizzative o procedurali, volte a perfezionare il sistema di sicurezza in azienda, può diventare elemento di ausilio nel fornire la prova di «aver fatto tutto il possibile per evitare il danno» ovvero di «aver adottato tutte le cautele necessarie per impedire il verificarsi del danno medesimo» ${ }^{125}$. È nota la formula giurisprudenziale che pone a carico del datore di lavoro, ai sensi dell'art. 1218 c.c., l'onere di dimostrare «che l'impossibilità della prestazione o la non esatta esecuzione della stessa o, comunque, il pregiudizio che colpisce la controparte derivano da causa a lui non imputabile» ${ }^{126}$. Ove si verifichi il caso di infortunio sul lavoro e fornita la prova da parte del lavoratore del nesso di causalità fra l'esistenza del danno e la nocività dell'ambiente di lavoro, il datore di lavoro che abbia adottato una buona prassi può più agevolmente dimostrare di aver assolto al dovere di sicurezza.

$\mathrm{Al}$ contrario, non si potrà non tenere conto del mancato utilizzo della prassi appropriata e conosciuta, o quantomeno conoscibile, da parte del datore di lavoro/debitore di sicurezza. Anche questa circostanza assume in definitiva rilievo. Se esiste una soluzione migliorativa delle condizioni di lavoro resa pubblica e disponibile, il datore di lavoro non può ignorarla. La verifica dell'adempimento degli ob-

125 Cass., sez. lav., 4 febbraio 2016, n. 2209, in DeJure; Cass., sez. lav., 17 febbraio 2009, n. 3788, in DeJure.

126 Cass., sez. lav., 5 febbraio 2016, n. 2326, in DeJure; nonché Cass., sez. lav., 11 aprile 2013, n. 8855, in DeJure; Cass., sez. lav., 28 agosto 2013, n. 19826, in DeJure. Sui profili di responsabilità ai sensi dell'art. 2087 c.c. cfr., in particolare, P. Albi, Sicurezza sul lavoro e sistema civile (2087 c.c.), in G. Natullo (a cura di), Salute e sicurezza sul lavoro, cit., p. 148 ss.; ID., Adempimento dell'obbligo di sicurezza e tutela della persona, cit., p. 246 ss.; A. LASSANDARI, Art. 2087 c.c., in O. Cagnasso, A. Vallebona (a cura di), Dell'impresa e del lavoro, Milano, Utet Giuridica, 2013, p. 181 ss. 
blighi prevenzionistici espressamente previsti dalla legge non può, quindi, prescindere dalla stessa verifica dell'esistenza e dell'adozione di tali buone prassi.

Questa lettura è, in un certo senso, ricavabile anche sulla scorta dell'indirizzo giurisprudenziale che ha ricondotto all'obbligo «generico» di sicurezza l'adozione di «comportamenti specifici», non contemplati dalla legge, bensì «suggeriti da conoscenze sperimentali e tecniche» ${ }^{127}$ o rintracciabili in «altre fonti analoghe» ${ }^{128}$.

In una recente decisione che desta peculiare interesse, è stata riconosciuta la responsabilità del datore di lavoro per non aver apprestato «tutte le misure idonee» a ridurre le conseguenze dell'evento dannoso, le quali all'epoca dei fatti non erano legislativamente imposte, ma comunque conosciute per essere state pubblicate dall'International Organization for Standardization (ISO) ${ }^{129}$. Soluzioni che, come accertato in giudizio, ove rispettate avrebbero, se non evitato il verificarsi dell'evento, quantomeno causato conseguenze di più ridotta entità.

Avendo la giurisprudenza attribuito valore alle norme tecniche approvate e divulgate da organizzazioni internazionali, «la cui osservanza non sia obbligatoria», come precisato nell'art. 2, comma 1, lett. u, d.lgs. n. 81/2008, riflessioni non dissimili potrebbero riguardare il mancato utilizzo delle stesse buone prassi, in particolare se validate dalla Commissione consultiva permanente con l'intento della loro più ampia diffusione.

Le considerazioni formulate risultano avvalorate se si prende in esame il meccanismo previsto nell'Health and Safety at Work Act rispetto ai già menzionati Approved Codes of Practices; come sarà me-

127 In tal senso v., già, Cass., sez. lav., 23 luglio 2004, n. 13887, in DeJure; Cass., sez. lav., 25 agosto 2003, n. 12467, in DeJure.

128 Cass., sez. lav., 30 giugno 2016, n. 13465, in DeJure; Cass., sez. lav., 5 gennaio 2016, n. 34, cit.; nonché Cass., sez. lav., 2 luglio 2014, n. 15082, cit., ove si precisa che, con riferimento a «misure di sicurezza innominate», la prova liberatoria a carico del datore di lavoro è correlata «alla quantificazione della misura di diligenza ritenuta esigibile». In giurisprudenza è stato, inoltre, affermato che il datore di lavoro ha l'onere di provare «di aver adottato tutte le cautele necessarie ad evitare il danno, in relazione alle specificità del caso ossia al tipo di operazione effettuata ed ai rischi intrinseci alla stessa, potendo al riguardo non risultare sufficiente la mera osservanza delle misure di protezione individuale imposte dalla legge»: v., ad esempio, Cass., sez. lav., 19 luglio 2007, n. 16003, in DeJure.

129 Cass., sez. lav., 30 giugno 2016, n. 13465, cit. 
glio precisato, infatti, la speciale regolazione ivi dettata consente al giudice di poterne tenere conto al fine dell'assolvimento dell'onere della prova in giudizio.

Significative sono, altresì, le conseguenze della dismissione di una buona prassi precedentemente adottata, nell'ipotesi che si verifichi un infortunio sul lavoro causalmente collegato alla regola cautelare omessa: la responsabilità datoriale sembrerebbe accertata $a$ fortiori.

Occorre, invece, ricordare che assumono valore di per sé vincolante le norme tecniche, nonché le buone prassi, se espressamente richiamate dallo stesso legislatore ${ }^{130}$. Le prime in particolare, cui il d.lgs. n. 81/2008 rinvia di frequente, sono assimilate alla legge ove, ad esempio, costituiscano il parametro di specificazione tecnica al fine di fornire una adeguata informazione ai lavoratori in merito ai pericoli connessi all'uso di sostanze pericolose, ai sensi dell'art. 36, d.lgs. n. 81. Le norme tecniche rilevano, inoltre, nell'ambito degli obblighi datoriali, «qualora sia necessario fornire mediante la segnaletica di sicurezza indicazioni relative a situazioni di rischio» non considerate negli allegati di cui al d.lgs. n. 81: anche «in riferimento alle norme di buona tecnica», il datore di lavoro «adotta le misure necessarie secondo la particolarità del lavoro, l'esperienza e la tecnica» (art. 163, comma 2, d.lgs. n. 81/2008).

Infine, qual è la vincolatività delle buone prassi dal punto di vista del diritto penale?

Un ultimo interrogativo sul quale la dottrina si è di recente soffermata concerne, in effetti, la valenza delle buone prassi rispetto ai principi penalistici ed ai profili di responsabilità penale. Tali buone prassi non sembrano del tutto irrilevanti nemmeno nella prospettiva penalistica.

Viene, in primo luogo, in rilievo il principio di legalità, posto che non vi può essere alcuna punizione per un fatto che non sia espressamente previsto per legge come reato (art. 25 Cost.; art. 1 c.p.), nonché, esattamente, il principio di riserva di legge, che affida al solo legislatore l'individuazione della fattispecie. La materia de qua rap-

130 Quanto alle buone prassi richiamate nel d.lgs. n. 81/2008 cfr. in particolare il paragrafo 1 . 
presenta il «terreno d'elezione» al fine di «intraprendere un'indagine sulle fonti del diritto e sul rapporto di tensione che si instaura fra il principio di legalità e le concrete esigenze di prevenzione» ${ }^{131}$.

Si deve considerare che le richiamate misure non sono in sé fonte di responsabilità penale; pertanto il mancato rispetto di tali regole migliorative non può, di per sé, dar luogo a responsabilità proprio in virtù del principio di legalità e di riserva di legge.

Tuttavia, le buone prassi potrebbero ugualmente assumere rilevanza, oltreché sotto il profilo civilistico, anche sul piano penalistico. Come chiarito, quanto esattamente all'inosservanza di regole cautelari contenute in buone prassi «richiamate da fonti cogenti», ossia dal d.lgs. n. 81/2008, tende a ripetersi lo schema della colpa specifica ${ }^{132}$ : la violazione di tali misure può fondare un rimprovero a titolo di colpa specifica ove ne consegua un evento dannoso e pericoloso. Il «settore della sicurezza del lavoro», del resto, si contraddistingue «per la tradizionale e maggiore rilevanza della colpa specifica (o per inosservanza di regole formalizzate) rispetto alla colpa generica (o per negligenza, imprudenza, imperizia) $\gg^{133}$.

Diversamente, pur trattandosi di un aspetto ancora dibattuto e controverso in dottrina ${ }^{134}$, in mancanza di un esplicito richiamo, os-

131 V. TORRE, La valutazione del rischio e il ruolo delle fonti private, in D. CAstronuovo, F. Curi, S. Tordini Cagli, V. Torre, V. Valentini, Diritto penale della sicurezza sul lavoro, Bologna, Bononia University Press, 2016, p. 44.

132 D. Castronuovo, Responsabilità colposa e sicurezza del lavoro. Dalla colpa alle colpe, in M. Mantovani, F. Curi, S. Tordini Cagli, V. Torre, M. Caianiello (a cura di), Scritti in onore di Luigi Stortoni, Bologna, Bononia University Press, 2016, p. 182, secondo cui, tuttavia, negli altri casi «i problemi legati alla non obbligatorietà di tali protocolli vanno risolti mediante riconduzione ai criteri di ricostruzione della colpa generica». Sul tema del rinvio espresso a fonti soft cfr., in particolare, A. BERNARDI, Sui rapporti tra diritto penale e soft law, in RIDPP, 2011, p. 574.

133 D. Castronuovo, Responsabilità colposa e sicurezza del lavoro. Dalla colpa alle colpe, cit., p. 170 e p. 174, secondo il quale si può configurare una colpa per inosservanza di discipline, ai sensi dell'art. 43 c.p. , «in presenza di una violazione di regole di sicurezza contenute in disposizioni aziendali», le quali siano «la specificazione di criteri generali di sicurezza desumibili dall'ordinamento giuridico» e ritagliate sulle specifiche situazioni di rischio. Cfr. in proposito P. TuluINI, Tutela civile e penale della sicurezza del lavoro: principi, categorie e regole probatorie a confronto, in RTDPC, 2011, p. 735.

134 Sulla problematica delle regole di condotta «generiche» especifiche»cfr. G. Marini, voce Colpa II) Diritto Penale, in Enc. Giur. Treccani, VI, Roma, 1988, 
sia in presenza di «regole cautelari che si elasticizzano», verrebbe in rilievo la colpa generica, «ideologicamente supportata dal rinvio all'art. 2087 c.c.» ${ }^{135}$.

\section{Spunti per una comparazione}

Le buone prassi sono, senza dubbio, incentivate a livello europeo in molti Stati membri, come testimonia l'iniziativa promossa e divulgata ormai da diversi anni dall'Agenzia europea per la sicurezza e la salute sul lavoro, pur mancando spesso, nelle discipline nazionali, una apposita definizione ed un sistema di validazione comparabile con quello italiano.

Considerata la loro notevole diffusione, vale la pena, a questo punto, soffermarsi in merito alla regolamentazione giuridica e tentare una, seppur breve, comparazione con alcuni Stati tra quelli maggiormente coinvolti nell'opera di elaborazione e raccolta delle buone prassi in subiecta materia.

Si deve premettere che il seguente confronto con gli ordinamenti di Spagna, Francia, Germania e Regno Unito, non è affatto agevole, considerate le notevoli peculiarità che caratterizzano, anzitutto, il sistema delle fonti proprio di quei paesi. Le difficoltà sono, altresì, acuite dalla scarna attenzione finora generalmente prestata da parte della dottrina al tema della prevenzione dei rischi nell'ambiente di lavoro, che sembra rimanere essenzialmente "di nicchia" nell'ampio panorama delle problematiche lavoristiche; poco spazio è stato, poi, specificamente dedicato alla formazione di regole volte a migliorare gli standard di protezione.

p. 6 ss. In merito agli «equilibri difficili tra giudizio penale e giudizio civile», nonché alla nozione di colpa nella tutela civile e penale della sicurezza sul lavoro cfr. le considerazioni di P. TulLini, Tutela civile e penale della sicurezza del lavoro: principi, categorie e regole probatorie a confronto, cit., p. 736; nonché EAD., La responsabilità civile per esposizione lavorativa all'amianto: obbligo di sicurezza, limiti dell'esonero assicurativo e risarcibilità del danno, in RDSS, 2016, n. 1, p. 48.

135 C. Piergallini, Colpa e attività produttive: un laboratorio di diritto "cedevole”, in G. Casaroli, F. Giunta, R. Guerrini, A. Melchionda (a cura di), La tutela penale della sicurezza del lavoro, Pisa, Edizioni ETS, 2015, p. 391. 
La considerazione preliminare attiene alla verifica della presenza o meno nei menzionati sistemi giuridici di un'apposita definizione e disciplina delle buone prassi, da poter confrontare con quella introdotta nel nostro ordinamento.

Per la verità, non è dato riscontrare rinvii significativi all'aspetto più moderno della regolazione volontaria della materia. Le normative nazionali prese in considerazione restano, per lo più, fortemente accomunate ed influenzate dai principi di carattere generale della direttiva comunitaria 89/391/CEE. Da questo punto di vista, è evidente, se non giustificato, «il grado di armonizzazione» tra gli ordinamenti, quantomeno rispetto ad alcuni principali profili $^{136}$.

Nonostante, il recepimento da parte degli Stati membri dei principi generali contenuti nella direttiva quadro europea ${ }^{137}$, si possono, tuttavia, riscontrare alcune evidenti differenze anche in ordinamenti assai simili, come ad esempio quello italiano e spagnolo. Si tratta, infatti, di discipline distinte, anche se sviluppatesi su valori comuni, adattate alle peculiari realtà nazionali, tra l'altro approvate in tempi diversi e non sempre ammodernate e novellate al pari di quella italiana. L'impressione che si può anticipare è, in effetti, che in particolare in Italia è stata avvertita la necessità di riordinare complessivamente il quadro normativo attraverso l'introduzione di nuove nozioni e l'ampliamento del suo campo di applicazione, pur residuando alcuni elementi di criticità.

\subsection{L'esperienza spagnola, francese e tedesca}

L'elemento che accomuna l'ordinamento spagnolo, francese e tedesco è, pur nella diversità delle singole esperienze nazionali, la

136 B. Caruso, Introduzione, in B. Caruso, M. D’Antona, S. Sciarra (a cura di), Il diritto del lavoro nell'Unione europea. Francia, Germania, Gran Bretagna, Spagna, cit., p. 167.

137 Quanto all'influenza della direttiva comunitaria sulle singole esperienze nazionali v. M. BIAGI (a cura di), Tutela dell'ambiente di lavoro e direttive Cee, Maggioli, Rimini, 1991; nonché R. BALdwin, T. Daintith, Harmonization and Hazard. Regulating Health and Safety in the European Workplace, London, Dordrecht, Boston, Graham \& Trotman, 1992. 
mancanza di una precisa nozione legislativa di buone prassi, in realtà presenti e variamente diffuse.

Quanto al sistema spagnolo il principale riferimento normativo è costituito dalla Ley de Prevención de Riesgos Laborales (31/1995), modificata ed integrata da successivi interventi legislativi, la quale, com'è stato fin da subito precisato, si limita generalmente a riprodurre i contenuti della direttiva quadro 89/391/ $\mathrm{CEE}^{138}$.

Tale modello di regolazione si articola, esattamente, in una parte preliminare di carattere generale (artt. 1-5), in una parte relativa all'ambito di applicazione ed alle definizioni, nella política en materia de prevención de riesgos para proteger la seguridad y la salud en el trabajo (capítulo II), nei diritti ed obblighi delle parti (capítulo III), oltreché nelle responsabilità e sanzioni (capítulo VII) ${ }^{139}$; tra i doveri del datore di lavoro v'è il costante controllo ed il miglioramento continuo dell'attività preventiva dei rischi (art. 14.2) $)^{140}$.

Particolare attenzione merita la consultazione e partecipazione dei lavoratori (capítulo V), garantita attraverso i Delegados de Prevención, ossia i rappresentanti dei lavoratori con specifiche funzioni in materia ed il Comité de Seguridad y Salud, organismo paritetico costituito nelle imprese che occupano almeno cinquanta dipendenti (art. 38). Tra le funzioni ad esso attribuite vi sono la partecipazione all'elaborazione dei piani di valutazione dei rischi, nonché la promozione di iniziative sui metodi e sulle procedure finalizzate ad una prevenzione efectiva, con la possibilità di propo-

138 Si tratta della Ley 31/1995, de 8 de noviembre, de Prevención de Riesgos Laborales. Cfr., in particolare, il commento di F. Pérez de los Cobos Orinuel, $L a$ ley de prevención de riesgos laborales como transposición de la directiva marco sobre seguridad y salud de los trabajadores en el lugar de trabajo, in Tribuna social, 1997, p. 7 ss.

139 Per completezza il capítulo IV è dedicato ai servicios de prevención e il capítulo VI alle obligaciones de los fabricantes, importadores y suministradores.

140 In ordine al carattere dinamico dell'obbligo di sicurezza ai sensi dell'art. 14.2 della Ley de prevención de riesgos laborales cfr. F.A. González Díaz, M. Cos Egea, Artículo 14. Derecho a la protección frente a los riesgos laborales, in A.V. Sempere Navarro (dir.), M. Cardenal Carro, I. Alzaga Ruiz (coord.), Comentarios a la Ley de Prevención de Riesgos Laborales, Navarra, Aranzadi, 2010, p. 172. 
sta di misure migliorative e correttive delle carenze esistenti (art. 39) ${ }^{141}$.

Come anticipato, non è invece contenuto nessun esplicito riferimento normativo a regole o strumenti volontari destinati ad elevare i livelli di sicurezza. La mancata introduzione nella legislazione spagnola di un'apposita nozione e disciplina di buone prassi è, però, colmata attraverso una loro divulgazione ad opera in particolare dell'Instituto Nacional de Seguridad e Higiene en el Trabajo, nonché delle associazioni imprenditoriali denominate Mutuas de Accidentes de Trabajo y Enfermedades Profesionales de la Seguridad Social $^{142}$. Non mancano, insomma, a livello nazionale esempi di buenas prácticas variamente diffusi ${ }^{143}$.

Non molto dissimili sono le riflessioni che si possono formulare in ordine al sistema francese di regolazione della tutela della sicurezza sul lavoro. La trasposizione nel diritto francese della direttiva comunitaria del 1989 è avvenuta nel 1991, in anticipo rispetto all’I-

141 In merito alla funzione ed ai compiti del Comité de Seguridad y Salud cfr. M.C. Aguilar del Castillo, El Comité de Seguridad y Salud, Valencia, Tirant Lo Blanch, 2013, p. 265 ss.; nonché EAD., El Comité de Seguridad y Salud como órgano de participación de los trabajadores en la negociación colectiva, in J. CABEZA Pereiro, B. Fernández Docampo, Participación y acción sindical en la empresa, Albacete, Bomarzo, 2014, p. 113 ss. Quanto agli aspetti partecipativi nella gestione della sicurezza sul lavoro si veda specificamente il capitolo III.

142 V., al riguardo, J. Aparicio Tovar, Occupational Health and Safety in Spain: A Formidable Array of Employers' Liabilities, in E. Ales (ed.), Health and Safety at Work. European and Comparative Perspective, Alphen aan den Rijn, Wolters Kluwer, 2013, p. 258. Si tratta di associazioni imprenditoriali autorizzate dal Ministero del lavoro e della previdenza sociale, costituite senza scopo di lucro al fine di cooperare, sotto la direzione e supervisione del Ministero, nella gestione dei rischi di infortuni e malattie professionali dei dipendenti dei datori di lavoro associati (v. art. 80.2, Texto Refundido de la Ley General de la Seguridad Social). Quanto ai modelli di buenas prácticas divulgati dalle Mutuas de Accidentes de Trabajo y Enfermedades Profesionales de la Seguridad Social corrispondono più propriamente a pubblicazioni di sensibilizzazione alla tematica della prevenzione dei rischi, relative a specifici settori produttivi o rischi, dunque manuali contenenti indicazioni o linee guida.

143 Si possono ad esempio menzionare le misure innovative intraprese volontariamente al fine di sensibilizzare e rafforzare la cultura della sicurezza da Heineken España, che nel 2013 ha introdotto un programma di formazione in formato e-learning, il c.d. «Health \& Safety Academy», con il risultato di una significativa riduzione del numero e della gravità degli infortuni sul lavoro: $w w w$.heinekenespana.com. 
talia e, soprattutto, entro i termini richiesti dalla normativa comunitaria $^{144}$. La materia della santé et sécurité au travail è contenuta nel Code du travail, in particolare nella parte quarta ${ }^{145}$.

Anzitutto è apprezzabile la tecnica legislativa adottata, posto che le regole prevenzionistiche, suddivise tra una parte legislativa e una regolamentare, sono inserite insieme alle altre all'interno del medesimo Codice che, per quanto articolato, costituisce un unico punto di riferimento, in ossequio a quella "semplicità" legislativa tanto agognata nel nostro ordinamento ${ }^{146}$. V'è, inoltre, da chiarire che, nonostante l'aggiornamento pressoché costante delle previsioni contenute nel Code du travail, la materia non ha subito un riassetto complessivo come invece avvenuto con il d.lgs. n. 81/2008. Forse anche per questo motivo, l'impostazione "tradizionale" del modello francese non contempla alcun esplicito richiamo alle buone prassi o a specifiche soluzioni migliorative dei livelli di sicurezza.

All'interno di tale quadro normativo l'attenzione è, in primo luogo, dedicata, così come negli altri Stati membri, agli obblighi delle parti e, in particolar modo, del datore di lavoro, individuato quale principale responsabile ${ }^{147}$. Come sottolineato, il datore di lavoro deve garantire le misure necessarie al fine di tutelare la salute fisica e mentale dei prestatori di lavoro, tenendo conto «dei mutamenti delle circostanze» e mirando al «miglioramento delle situazioni esistenti» (Quatrième partie, Livre Ier, Titre II, Principes généraux de prévention, art. L 4121-1); in seguito alla valutazione dei rischi, deve, inoltre, mettere in atto azioni preventive e metodi di lavoro e della produzione al fine di garantire «un meilleur nive-

144 I principi della direttiva quadro 89/391/CEE sono stati recepiti dalla Loi n. 91-1414 du 31 décembre 1991, Modifiant le Code du travail et le code de la santé publique en vue de favoriser la prévention des risques professionnels et portant transposition de directives européennes relatives à la santé et à la sécurité du travail.

145 Cfr.: www.legifrance.gouv.fr.

146 Si veda, tuttavia, anche il Code de la sécurité sociale, che contiene le norme relative al diritto della sicurezza sociale e, tra queste, le regole in materia di riparazione economica degli infortuni sul lavoro e delle malattie professionali.

147 Per un commento in merito ai doveri delle parti v., in particolare, C. SAchs-Durand, Occupational Health and Safety in France: A Good Formal Protection, but a Problematic Efficiency, in E. Ales (ed.), Health and Safety at Work. European and Comparative Perspective, cit., p. 110 ss. 
au de protection de la santé et de la sécurité des travailleurs» (art. L 4121-3).

Il Titre IV si riferisce alle «Istituzioni» e «persone che contribuiscono all'organizzazione della prevenzione», in particolare, alla Agence nationale pour l'amélioration des conditions de travail, la quale, ai sensi dell'art. L 4642-1, ha lo scopo precipuo di contribuire allo sviluppo ed alla promozione di esperienze o risultati nell'ottica del miglioramento delle condizioni di lavoro, nonché di raccogliere e diffondere le relative informazioni in Francia e all'estero. Si tratta, in particolare, di un organismo governato da un consiglio, composto in numero uguale dai rappresentanti delle organizzazioni datoriali a livello nazionale, dai rappresentanti delle organizzazioni sindacali dei lavoratori a livello nazionale e dai rappresentanti dei Ministeri interessati e da persone qualificate, che quanto alla sua formazione ricorda in parte la Commissione consultiva permanente di cui all'art. 6 , d.lgs. n. 81/2008 ${ }^{148}$. Tale ente pubblico, incardinato sotto il Ministero del lavoro e costituito da un «consiglio d'amministrazione tripartito», ha come vocazione quella di «promuovere, facilitare e trasferire politiche, strumenti e metodi al fine di elevare la prevenzione nell'ambiente di lavoro» ${ }^{149}$. L'attività svolta dall'Agence nationale si caratterizza tra l'altro per la raccolta e diffusione di "casi aziendali", alcuni dei quali dedicati a tematiche relative alla prevenzione dei rischi sul lavoro. Esempi significativi di cas d'entreprise sono quelli che mirano a realizzare un piano d'azione al fine di ridurre le disfunzioni legate ai rischi psicosociali ovvero a porre la partecipazione dei lavoratori al centro delle relazioni industriali ${ }^{150}$. Si tratta, verosimilmente, di azioni o soluzioni realizzate su singole problematiche concernenti l'organizzazione e le condizioni di lavoro, in un certo senso assimilabili a buone prassi, presentate, divulgate e sostenute dall'Agence nationale.

148 Cfr. l'art. L 4642-2, come modificato dalla Loi organique n. 2010-704 du 28 juin 2010 - art. 21, ove è precisato che «participent au conseil d'administration, à titre consultatif, un représentant de chacune des commissions chargées des affaires sociales au Parlement, ainsi qu'un représentant de la section chargée des affaires sociales au Conseil économique, social et environnemental».

149 Cfr. www.anact.fr.

150 La casistica è reperibile in www.anact.fr. 
Spetta, invece, al Ministero del lavoro la diffusione dei criteri per poter partecipare alla competizione organizzata dall'Agenzia europea per la sicurezza e la salute sul lavoro, nonché degli scopi che le stesse pratiche devono perseguire: è stato pertanto predisposto un modello di presentazione delle soluzioni volontarie costruito secondo lo schema europeo ${ }^{151}$.

Tra gli attori della prevenzione, merita particolare attenzione anche il Conseil d'Orientation sur les Conditions de Travail, organismo alle dipendenze del Ministero del lavoro, subentrato al Conseil supérieur de la prévention des risques professionnels. Il Consiglio è coinvolto nello sviluppo delle politiche nazionali per la prevenzione dei rischi, consultato in merito a progetti di legge in materia di tutela e promozione della salute e sicurezza sul lavoro ed ha il potere di formulare suggerimenti e proposte sulle condizioni di lavoro ${ }^{152}$.

Un ruolo importante nel contribuire a migliorare la tutela della salute dei prestatori di lavoro è, altresì, attribuito al Comité d'hygiène, de sécurité et des conditions de travail ${ }^{153}$, presente negli stabilimenti con almeno cinquanta dipendenti e composto da rap-

151 Cfr. www.travail-emploi.gouv.fr. Tra gli esempi concreti riportati dall'Agenzia europea per la sicurezza e la salute sul lavoro, che illustrano soluzioni messe in opera al fine di risolvere determinate problematiche, si veda quello della Ferrero (sede francese), che nel 2007 ha lanciato un programma chiamato «Ferrero Ensemble Justement», avente ad oggetto misure specifiche di promozione della salute nei luoghi di lavoro. La «strategia del benessere» promossa dalla «Ferrero France» comprende, tra le altre, iniziative finalizzate a ridurre i rischi conseguenti alla circolazione dei veicoli aziendali, mediante il rinnovo del parco veicoli e dei dispositivi di sicurezza ogni due anni, nonché una formazione per 4000 ore dei lavoratori sulla sicurezza stradale. Tra i fini della citata strategia v'è il proposito della trasferibilità delle misure utilizzate presso le altre sedi della Ferrero in Europa, Italia compresa.

152 Il Conseil d'Orientation sur les Conditions de Travail è regolato nel Code du travail (art. L 4641-1 ss.; art. R 4641-1 ss.) ed è costituito da componenti dei Ministeri interessati, da un ugual numero di rappresentanti dei lavoratori e dei datori di lavoro, da persone qualificate e da rappresentanti delle associazioni o organismi di prevenzione.

153 Il Comité d'hygiène, de sécurité et des conditions de travail è preso in esame nel capitolo III. Istituito dalla legge n. 1097 del 23 dicembre 1982, il c.d. «pacchetto Auroux», è regolato nel Livre VI, Institutions et Organismes de Prévention, Titre Ier. Modifiche all'istituto sono preannunciate nel progetto di Ordonnance relative à la nouvelle organisation du dialogue social et économique dans l'entreprise et favorisant l'exercice et la valorisation des responsabilités syndicales, presentato il 31 agosto 2017. 
presentanti del personale e dal datore di lavoro, che ricorda il $\mathrm{Co}$ mité de Seguridad y Salud; tra i compiti ad esso assegnati, v’è la promozione di tutte le iniziative considerate utili ai fini prevenzionistici (art. L 4612-3), ovvero in sostanza «il diritto ed il dovere di contribuire alla prevenzione dei rischi professionali» nella direzione di una effettiva consultazione e partecipazione dei lavoratori, per il tramite delle loro rappresentanze ${ }^{154}$.

Dunque, vi sono diversi «organismi di prevenzione» cui il sistema francese affida il compito di contribuire, in vario modo, ad aumentare la protezione dei lavoratori in azienda.

È, poi, opportuno soffermarsi sulle specificità che presenta il modello tedesco, caratterizzato da una «obiettiva complessità del sistema delle fonti» che concorrono a delineare il quadro della normativa prevenzionale ${ }^{155}$.

La legislazione di riferimento è contenuta nel $\operatorname{ArbSch} G$, adottato dal Parlamento federale nel 1996 e successivamente modificato, che stabilisce i principali obblighi dei datori di lavoro e dei lavoratori in materia di salute e sicurezza sul lavoro ${ }^{156}$. Il sistema tedesco è, però, un sistema duale, ove sono presenti diverse istituzioni cui l'ordinamento attribuisce il potere di creare regole in questo ambito, rilevando, in particolare, le Berufsgenossenschaften, ossia le Associazioni categoriali di assicurazione contro gli infortuni sul lavoro ${ }^{157}$. Questi organismi, come precisato nel Sozialgesetzbuch VII (il Settimo Libro del Codice Sociale), oltre a dover provvedere con tutti i

154 Tale organismo, come precisato, «gioca un ruolo di primo piano nella politica di prevenzione dei rischi professionali all'interno dell'impresa»: M. DesPaX, J. Rojot, J.P. Laborde, Labour law in France, Alphen aan den Rijn, Wolters Kluwer, 2011, p. 265. Cfr., altresì, in proposito J. Pélissier, G. Auzero, E. Dockès, Droit du travail, Paris, Dalloz, 2012, p. 882 ss.; A. Mazeaud, Droit du travail, Paris, Montchrestien, 2010, p. 146 ss.

155 A. Lo Faro, Germania, in B. Caruso, M. D’Antona, S. Sciarra (a cura di), Il diritto del lavoro nell'Unione europea. Francia, Germania, Gran Bretagna, Spagna, cit., p. 316 ss.

156 L'Arbeitsschutzgesetz (legge concernente l'attuazione di misure di sicurezza sul lavoro e per migliorare la sicurezza e la salute dei lavoratori durante il lavoro) del 7 agosto 1996 è stato da ultimo modificato nel 2015, precisamente da Artikel 427 der Verordnung vom 31 August 2015: www.gesetze-im-internet.de/arbschg.

157 Cfr., al riguardo, le considerazioni di O. Deinert, Occupational Health and Safety in Germany: a Dual System in Change, in E. Ales (ed.), Health and Safety at Work. European and Comparative Perspective, cit., p. 127 ss. 
mezzi idonei alla tutela della sicurezza sul lavoro e indagare sui fattori scatenanti i pericoli per la salute dei lavoratori, hanno, appunto, il potere di adottare misure vincolanti per il datore di lavoro, al fine della prevenzione degli infortuni ( $§ \S 14$ e 15 SGB VII $)^{158}$.

Rileva, altresì, la previsione di una strategia nazionale per la sicurezza sul lavoro (Gemeinsame Deutsche Arbeitsschutzstrategie), alla quale partecipano diversi attori con il fine del perseguimento di obiettivi comuni orientati alla tutela del lavoro nell'impresa ed alla diffusione di una politica di prevenzione duratura nel tempo ( $\S \S 20 \mathrm{a}$ e $20 \mathrm{~b} A r b S c h G)^{159}$. È esattamente dal 2008 che lo Stato, le Regioni e gli Enti assicurativi collaborano per migliorare ed incentivare la salute e la sicurezza sul lavoro, promuovendo nell'ambito della menzionata strategia, ogni due anni, un premio per la tutela del lavoro, specificamente rivolto alle imprese che hanno introdotto processi e tecnologie innovative o misure migliorative dei livelli di sicurezza sul lavoro ${ }^{160}$. Gli organismi promotori sono il Ministero federale per il lavoro e gli affari sociali (Bundesministerium für Arbeit und Soziales), il Comitato delle Regioni per la tutela del lavoro e della sicurezza (Länderausschuss für Arbeitsschutz und Sicherheitstechnik) e l'Assicurazione obbligatoria tedesca per gli infortuni sul lavoro (Deutsche Gesetzliche Unfallversicherung).

A livello nazionale è, in tal modo, promossa e premiata la progettazione e presentazione da parte delle imprese, di qualunque settore e dimensione, di esempi di buone prassi, cioè di metodi orga-

158 Più esattamente le Berufsgenossenschaften, come precisato nel $\S 114$ del Sozialgesetzbuch VII, fanno parte dei Unfallversicherungsträger ed hanno il potere di adottare regole integrative, le Unfallverhütungsvorschriften, volte a specificare il contenuto della normativa prevenzionale di carattere generale: si veda al riguardo T. Klebe, Dritter Abschnitt: Soziale Angelegenheiten, in W. Däubler, M. KitTNER, T. KleBE, P. Wedde (hrsg.), Betriebsverfassungsgesetz. Kommentar für die Praxis mit Wahlordnung und EBR-Gesetz, Frankfurt am Main, Bund Verlag, 2014, p. 1763 ss. In merito ai $\S \S 14$ e 15 SGB VII cfr. in particolare M. KitTNER, R. PIEPER, Arbeitsschutzrecht, Frankfurt am Main, Bund Verlag, 2002, p. 592 ss.

159 V. per un commento U. FABER, W. KOHTE, Vorbemerkungen zu §§ 20a, 20b ArbSchG, in W. Kohte, U. Faber, K. Feldhoff (hrsg.), Gesamtes Arbeitsschutzrecht, Baden-Baden, Nomos, 2014, p. 362 ss.

$160 \mathrm{Si}$ tratta di un premio economico assegnato in seguito ad una selezione delle migliori pratiche da parte di una giuria composta da un gruppo di esperti indipendenti provenienti dal mondo politico, economico, scientifico e accademico: www.deutscher-arbeitsschutzpreis.de. 
nizzativi e soluzioni tecniche, la cui successiva pubblicazione vale come incentivo per la loro adozione anche in altri contesti. Questi «Best-Practice Beispiele» devono, inoltre, rispettare determinati criteri, quali l'efficienza ed economicità, la loro effettiva applicazione, l'innovatività e la trasferibilità ${ }^{161}$.

In analogia con l'ordinamento italiano, come precedentemente osservato, il modello di presentazione in esame richiede, oltre ai dati che connotano l'azienda che ha progettato la soluzione innovativa, la descrizione della sua realizzazione e dei successi ottenuti, come il miglioramento della salute dei lavoratori, la riduzione degli infortuni sul lavoro, nonché la diminuzione degli stessi costi per l'impresa.

L'iniziativa dell'Agenzia europea per la sicurezza e la salute sul luogo di lavoro è, così, replicata a livello interno con la riproposizione dei medesimi requisiti e finalità.

L'assenza di una disciplina ad hoc che regolamenti queste iniziative volontarie, per lo più datoriali, e ne certifichi gli effetti, non ne ha affatto impedito la promozione e diffusione, secondo parametri uniformi a livello europeo.

Va, infine, osservato che, similmente ai paesi presi in considerazione e oggetto di comparazione, il Bundesministerium für Arbeit und Soziales si premura di divulgare le modalità di partecipazione al concorso promosso dall'Agenzia europea, valutando previamente le domande ammissibili.

Pare, allora, particolarmente avvertita la necessità di perseguire la cultura della sicurezza attraverso l'adozione di misure volon$\operatorname{tarie}^{162}$.

161 Esempi di buone prassi sono, in particolare, diffusi dal Bundesministerium für Gesundheit e dalla Deutsche Gesetzliche Unfallversicherung: www.bmg. bund.de e www.dguv.de.

162 L’importanza delle «freiwilligen Maßnahmen» è opportunamente dimostrata in seguito ad un'indagine svolta nel 2013 dall'Associazione tedesca delle Camere di Commercio e Industria (Deutschen Industrie - und Handelskammertages), secondo cui il 90\% delle imprese ha adottato misure volte al miglioramento della salute dei prestatori di lavoro; quale conseguenza dal 1995 al 2013 il numero di infortuni sul lavoro è diminuito del $50 \%$ (www.arbeitgeber.de). 
8.2. Gli Approved Codes of Practice e le guidances nell'ordinamento britannico

Con riguardo alla promozione e diffusione di regole soft e di strumenti non legislativi risulta, in particolar modo, utile e proficuo prendere in considerazione il sistema britannico di tutela della salute e della sicurezza sul lavoro, al quale l'ordinamento italiano e il diritto comunitario stesso si sono, in un certo senso, ispirati ${ }^{163}$.

Questa branca del diritto nel Regno Unito consta, a dire il vero, di un corpus regolativo complesso, costituito dalla legge e dal common law, nonché, per alcuni aspetti, dal criminal law. Il riferimento normativo principale è più precisamente l'Health and Safety at Work Act (HSWA) del 1974, successivamente modificato, cui si aggiunge un vasto numero di dettagliate Regulations, deputate a disciplinare specifiche situazioni di rischio ${ }^{164}$.

La direttiva 89/391/CEE è stata, in seguito, attuata con le Management of Health and Safety at Work Regulations 1999, che hanno definito gli obblighi in capo al datore di lavoro di valutare, evita-

163 Il riferimento a regole soft ed al concetto di soft law, diffusosi e fatto proprio dal diritto comunitario e dai diritti nazionali, è, in verità, assai indeterminato e di non facile definizione; con esso si allude a strumenti o atti differenti rispetto alle fonti normative tradizionali per la mancanza all'origine di valenza o forza vincolante, pur non essendo privi di efficacia giuridica indiretta o de facto: si veda la ricostruzione del significato di soft law di L. SEnden, Soft law in European Community Law, Oxford and Portland, Hart Publishing, 2004, p. 111 ss.

164 L'Health and Safety at Work Act fu emanato nel 1974, in seguito all'incarico affidato nel 1970 al Comitato di inchiesta per lo studio e il miglioramento delle condizioni di lavoro, presieduto da Lord Robens e culminato con il Robens Report: cfr. Lord Robens, Safety and Health at Work. Report of the Committee 1970-72, London, HMSO, 1972. Per un primo commento cfr., in particolare, R.C. BROWNE, Safety and health at work: the Robens Report, in British Journal of Industrial Medicine, 1973, p. 87 ss. In merito ai general duties contenuti nell'HSWA cfr. V. HoWES, Duties and Liabilities under the Health and Safety at Work Act 1974: A Step Forward?, in Industrial Law Journal, 2009, vol. 38, n. 3, p. 306 ss. Occorre, altresì, precisare che l'applicazione dell'HSWA alle diverse nazioni che compongono il Regno Unito (Inghilterra, Scozia, Galles e Irlanda del Nord) è regolata nella section 84, «Extent and application of Act», che, nel dettare alcune esclusioni, assicura l'estensione della parte I, contenente i principi sostanziali in materia di salute e sicurezza sul lavoro a tutto il Regno Unito (Part I «Health, Safety and Welfare in connection with Work, and Control of Dangerous Substances and Certain Emissions into the Atmosphere»). 
re e ridurre i rischi lavorativi, nell'ambito del quadro regolativo già previsto dall'Health and Safety at Work Act.

Dopo aver regolato i general duties delle diverse parti coinvolte a vario titolo nell'adempimento dell'obbligo di sicurezza, l'HSWA dedica la section 16 all'approvazione di Codes of Practices da parte dell'Health and Safety Executive (HSE) ${ }^{165}$, al fine di fornire indicazioni pratiche, esattamente una «practical guidance», di supporto alle norme vigenti. Senza dubbio un ruolo fondamentale nella promozione della salute e della sicurezza nei luoghi di lavoro è svolto dall'Health and Safety Executive (HSE), che negli anni recenti ha compiuto significativi progressi proprio nel contribuire ad incentivare best practices all'interno delle imprese ${ }^{166}$.

In linea generale, i Codes of Practice, nell'ambito delle fonti del diritto del lavoro britannico, detengono la funzione, oltreché di «codificare pratiche esistenti» in specifici settori, di «promuovere attivamente quelle considerate più opportune», con un «evidente impatto» nell'ambiente di lavoro ${ }^{167}$. Come è stato chiarito, questi Codes non sono vincolanti, anche se in alcuni casi le raccomandazioni ivi contenute sono state elevate al rango di «legal principles» ${ }^{168}$.

Con particolare riguardo all'ambito della tutela della salute, questi strumenti rispondono alla tendenza perseguita dall'International Labour Organization a limitare il ricorso alle fonti legislative e ad incoraggiare la realizzazione da parte delle autorità pubbliche di «practical guidelines», che non hanno forza di leg-

165 L'Health and Safety Executive è un ente pubblico istituito dall'HSWA, la cui funzione primaria è quella di garantire la salute, la sicurezza ed il benessere delle persone sul lavoro. È responsabile per la regolamentazione della salute e sicurezza sul lavoro in Gran Bretagna: www.hse.gov.uk; quanto all'Irlanda del Nord è responsabile l'HSE Northern Ireland: www.hseni.gov.uk.

166 In proposito si veda Common Sense Common Safety. A report by Lord Young of Graffham to the Prime Minister following a Whitehall-wide review of the operation of health and safety laws and the growth of the compensation culture, october 2010, ove sono contenute alcune proposte di revisione della materia e si precisa, in particolare, che «there is more that can be done to ensure that businesses are able to understand and comply with their responsibilities and respond in a proportionate way to health and safety requirements».

167 S. Deakin, G.S. Morris, Labour Law, Oxford and Portland, Hart Publishing, 2012, p. 63.

168 S. Deakin, G.S. Morris, Labour Law, cit., p. 63; nonché S. Hardy, Labour Law in Great Britain, Alphen aan den Rijn, Wolters Kluwer, 2012, p. 78. 
ge ma possono essere rapidamente adattate allo sviluppo tecnologico ${ }^{169}$.

Come anticipato, è lo stesso Health and Safety at Work Act che attribuisce all'HSE il potere di approvare ed emettere $A C O P$ s, di rivederli di volta in volta, anche solo in alcune parti, di avallare qualsiasi revisione o proposta di revisione, nonché di ritirare, in ogni momento, la sua approvazione. È ulteriormente specificata anche la loro natura giuridica; ai sensi della section 17 , infatti, la mancata osservanza delle regole degli $A C O P$ s non comporta di per sé l'assoggettabilità a procedimenti civili o penali. Anche se la violazione non è perseguibile civilmente o penalmente, la commissione di un reato con violazione di un obbligo o di un divieto contemplato negli stessi Codes assume però valore, posto che «qualsiasi previsione del codice di pratiche, che appare al giudice rilevante per l'obbligo o il divieto che si presume sia stato violato, è ammissibile come prova nel procedimento ${ }^{170}$. Seppur non giuridicamente vincolanti, gli ACOPS possono, allora, influenzare l'esito del giudizio; sono, infatti, ammissibili come prova contro il datore di lavoro responsabile, a meno che il giudice stesso non ritenga soddisfatto in altro modo l'obbligo di sicurezza. È, tuttavia, alquanto complesso prospettare l'effettiva implicazione derivante dalla mancata adozione di tali strumenti.

Questi principi sono ripresi dall'HSE nelle sue pubblicazioni, ove è riportata anche una, invero, labile distinzione tra $A C O P S$ e guidances, che lascia spazio ad alcuni dubbi ed incertezze, avvalorati dalla mancanza di un'approfondita riflessione da parte della dottrina.

Quanto ai primi è rimarcato lo status giuridico speciale che li contraddistingue, rimanendo possibile utilizzare metodi alternativi da quelli indicati al fine di rispettare le disposizioni normative; tuttavia, l'aver seguito le indicazioni contenute negli ACOPs è valuta-

169 Cfr. J.M. Servais, International Labour Law, Alphen aan den Rijn, Wolters Kluwer, 2011, p. 103 ss., ove sono menzionati alcuni Codes of practice pubblicati dall'ILO in ordine a peculiari situazioni di rischio, con la precisazione che si tratta di testi contenenti dettagliate guide pratiche, senza che si creino «legal obligations».

170 Così è possibile tradurre la section 17, subsection 2, dell'Health and Safety at Work Act. 
to come adempimento sufficiente rispetto alle prescrizioni di legge, naturalmente in relazione alle questioni specifiche sulle quali il Codice dà consigli.

In modo non dissimile, è esplicitato il carattere volontario delle guidances, emesse dall'HSE ed alle quali gli ispettori possono fare riferimento nel controllare l'osservanza delle disposizioni in materia di tutela della sicurezza nei luoghi di lavoro. Esattamente le guidances forniscono consigli, spiegazioni, informazioni tecniche specifiche, se non riferimenti a ulteriori fonti di informazione, quale ausilio al corretto adempimento degli obblighi di legge. Gli ACOPs descrivono e raccomandano i metodi preferibili, nonché gli standard che possono essere raggiunti, per rispettare gli obblighi imposti dall'Health and Safety at Work Act. Entrambi corrispondono, in un certo qual modo, a strumenti che offrono alle imprese la flessibilità di adattare le regole prevenzionistiche alle peculiari circostanze dell'ambiente di lavoro e alle condizioni di specifiche attività aziendali.

Benché, in ordine alla definizione ed al contenuto, sia spesso sottile la distinzione tra guidances e ACOPs, è vero che, a differenza delle guidances emanate esclusivamente dall'HSE, gli ACOPs, come sottolineato nella section 16 dell' $H S W A$, possono essere approvati dall'HSE, anche se non dallo stesso redatti o proposti.

Osservando le espressioni usate nei testi di alcune guidances si intuisce come esse rappresentino o contengano proprio le buone prassi: è, infatti, spesso precisato che «the guidance represents what is considered to be good practice» ${ }^{171}$. Le guide, essenzialmente rivolte ai datori di lavoro ed elaborate conformemente alle previsioni legislative, tendono ad offrire loro «good practice advice» e a descrivere case studies basati su particolari problematiche ${ }^{172}$. Così formulate le guidances sembrano, in effetti, molto simili alle buone

171 Cfr., ad esempio, Infection risks to new and expectant mothers in the workplace. A guide for employers, 1997, predisposta di concerto con l'HSE, dall'Advisory Committee on Dangerous Pathogens; nonché Your essential guide to health and safety in agriculture, una guida elaborata nel 2013 per tutti coloro che lavorano nelle aziende agricole, utile al fine di raggiungere buoni standard di salute e sicurezza.

172 Si veda, in particolare, Involving your workforce in health and safety. Good practice for all workplaces, elaborata nel 2008 e successivamente modificata nel 
prassi disciplinate e rese pubbliche nell'ordinamento italiano. Del resto, la stessa Agenzia europea per la sicurezza e la salute sul luogo di lavoro, nel fornire informazioni quanto alla definizione di buone prassi accolta dagli Stati membri, ha rinviato alle guidances del Regno Unito ${ }^{173}$.

Vero è, però, che non tutte le guidances sono tra loro uguali. Non mancano, in effetti, esempi di pubblicazioni che contengono nel medesimo testo insieme alle Regulations, dunque ai doveri legali, l'ACOP e la guidance, la quale, in questi casi si limita a riportare esemplificazioni, spiegazioni e consigli pratici su come adempiere alle disposizioni normative ${ }^{174}$. Si può finanche sostenere che, così formulata, la guidance rispecchi più propriamente il contenuto delle linee guida, disciplinate nell'ordinamento italiano all'art. 2, lett. z, d.lgs. n. 81/2008, ossia degli «atti di indirizzo e coordinamento per l'applicazione della normativa in materia di salute e sicurezza», predisposti dai Ministeri, dalle Regioni, dall'Inail ed approvati in sede di Conferenza permanente per i rapporti tra lo Stato, le Regioni e le Province autonome di Trento e di Bolzano.

Come anticipato, agli ACOPs e alle guidances, sarebbero, quantomeno in astratto, riconducibili le buone prassi fin qui considerate. Non si possono, tuttavia, non ricordare le peculiarità di queste ultime, quali soluzioni in prevalenza aggiuntive per meglio adempiere all'obbligo di sicurezza, rispetto agli strumenti propri del Regno Unito, per lo più codificati, cioè realizzati e/o pubblicati dall'HSE ed utilizzati quali termine di paragone per misurare l'adempimento della legge ${ }^{175}$. Di conseguenza, ad un attento esame, la coincidenza

2015, volta a cogliere la ratio del buon coinvolgimento dei lavoratori e delle loro rappresentanze sulla salute e sicurezza nei luoghi di lavoro.

173 L’Agenzia europea sottolinea, però, che il significato di «buone prassi» varia tra i diversi Stati membri, «per la diversità dei sistemi e delle norme sulla sicurezza e la salute sul luogo di lavoro, per la diversa cultura, lingua e le diverse esperienze»: http://osha.europa.eu/it/practical-solutions/it_good-practice_2009.pdf.

$174 \mathrm{Si}$ veda in questi termini Managing and working with asbestos. Control of Asbestos Regulations 2012. Approved Code of Practice and guidance, pubblicazione del 2013, in materia di esposizione al rischio amianto.

175 Tra le pubblicazioni dell'HSE vi sono anche guidances di carattere generale riferentisi alle misure che piccole e medie imprese possono mettere in atto per controllare i rischi sul lavoro: v. The health and safety toolbox. How to control risks at work, 2014, che contiene consigli su come adempiere più facilmente alle previ- 
tra guidances e good practices, come riconosciute a livello europeo, può venire a mancare, mentre con gli $A C O P$ s rimane, per lo più, una mera assonanza quanto alle espressioni che li identificano. La sensazione è, allora, che gli ACOPs e le guidances non mirino realmente ad un effettivo miglioramento dei livelli di tutela dei prestatori di lavoro, ma piuttosto alla corretta attuazione delle prescrizioni normative. In definitiva, mancherebbe l'elemento dell'innovatività, che caratterizza fortemente le buone prassi non solo in Italia, ma anche, come osservato, in Germania.

Occorre ulteriormente chiarire che, al di là della possibile commistione tra i diversi strumenti regolativi (ACOPs e guidances), che certamente crea qualche confusione in ordine alla loro configurazione dal punto di vista qualificatorio, nel sistema britannico è stata avvertita, ormai da qualche tempo, la necessità di riordinare e semplificare sia le numerose Regulations sia gli $A C O P$ s, eccessivamente lunghi, complessi e tecnici, nonché le guidances, perseguendo l'obiettivo di rendere più efficace l'attuale regolamentazione in materia di tutela della salute e sicurezza sul lavoro ${ }^{176}$. Al riguardo, le proposte di ridefinizione dei citati documenti emessi dall'HSE hanno suggerito, quanto agli ACOPs, di contenere informazioni non ambigue, bensì aggiornate rispetto ai cambiamenti della tecnologia e presentate nel modo più appropriato, quanto invece alle guidances, tra le altre, di evitare duplicazioni non necessarie ${ }^{177}$.

sioni normative in materia. Similmente a quanto sostenuto dall'Agenzia europea, è precisato che la salute e la sicurezza nei luoghi di lavoro «involves practical steps that protect people from harm and at the same time protect the future success and the growth of your business»; "good practice in health and safety makes sound business sense».

176 Cfr., in particolare, Reclaiming health and safety for all: an independent review of health and safety legislation, redatto nel novembre 2011 dal professor R.E. Löfstedt, al quale è stato affidato il compito da parte del Minister for Employment di redigere un «independent review of health and safety regulations to identify opportunities to simplify the rules». In merito al ruolo ed all'utilità degli ACOPs si veda la pubblicazione del 2001 dell'HSE Reducing risks, protecting people.

177 Reclaiming health and safety for all: an independent review of health and safety legislation, cit., p. 56. Cfr., inoltre, R.E. LÖFSTEDT, Reclaiming health and safety for all: a review of progress one year on, January 2013. 
È stato, altresì, sottolineato da parte della dottrina come la materia della prevenzione dei rischi nel Regno Unito si trovi «ad un bivio», essendo sempre più acceso il dibattito intorno all'utilità della vigente legislazione considerata «eccessivamente protettiva» e contenente «indebite restrizioni nei confronti delle imprese» ${ }^{178}$. Significativo è, da questo punto di vista, il programma di riforma progettato al fine di ridurre la burocrazia per le imprese ed infondere un «common sense» nell'applicazione della legislazione in materia di salute e sicurezza ${ }^{179}$.

Le raccomandazioni («Reclaiming health and safety for all: an independent review of health and safety legislation») sollecitate dal Minister for Employment sono state raccolte ed attuate dall'HSE. In particolare il documento del Departement for Work and Pensions intitolato «A final progress report on implementation of health and safety reforms» del marzo 2015, dà atto delle misure intraprese ${ }^{180}$. Tra queste la revisione e semplificazione degli $A C O P$ s esistenti attraverso il loro accorpamento, riscrittura, aggiornamento o finanche eliminazione.

178 M. Bell, Occupational Health and Safety in the UK: At a Crossroads?, in E. Ales (ed.), Health and Safety at Work. European and Comparative Perspective, cit., p. 375 ss., il quale riporta l'espressione popolare «health and safety gone mad».

179 Cfr. Common Sense Common Safety. A Report by Lord Young of Graffham to the Prime Minister following a Whitehall-Wide Review of the Operation of Health and Safety Laws and the Growth of Compensation Culture, cit.; nonche Good Health and Safety, Good for Everyone. The next steps in the Government's plans for reform of the health and safety system in Britain. Department for Work and Pensions, 21 March 2011.

180 Il documento «A final progress report on implementation of health and safety reforms» è reperibile in www.gov.uk. 
CAPITOLO II

\section{LA FORMAZIONE DI REGOLE IN AZIENDA TRA CONTRATTAZIONE E PARTECIPAZIONE}

Sommario: 1. Il ruolo della contrattazione e della partecipazione nella formazione di regole. - 2. La promozione della salute nel lavoro attraverso la contrattazione collettiva aziendale. -3 . Natura ed efficacia delle misure prevenzionistiche contrattualizzate. -4 . Contrattazione collettiva e prevenzione dei rischi nell'ordinamento spagnolo: un esempio affine. - 5. La «partecipazione equilibrata» nel contesto italiano. - 5.1. Il rappresentante dei lavoratori per la sicurezza.

1. Il ruolo della contrattazione e della partecipazione nella formazione di regole

È opportuno riflettere sulla possibilità che, a livello d'azienda, regole ulteriori ed aggiuntive rispetto alla disciplina prevenzionistica di cui al d.lgs. n. 81/2008 siano create ex novo in seno alla contrattazione collettiva e attraverso la partecipazione, rectius il coinvolgimento dei lavoratori e/o loro rappresentanze. Si tratta, in sostanza, della formazione di prassi condivise tra le parti e non solo frutto della decisione unilaterale del datore di lavoro.

Come noto, tale prospettiva ha da sempre suscitato forti dubbi e perplessità a fronte dello scarso interesse o ritrosia delle parti sociali nel prendere in considerazione la materia de qua, che mal si presta ad essere negoziata, nonché della mancanza nel nostro ordinamento 
di una «filosofia partecipativa» ${ }^{1}$, peraltro presente altrove, come si cercherà di chiarire.

Dunque, pur non potendosi parlare di un netto e soprattutto generale cambiamento di rotta rispetto al recente passato, vale la pena soffermarsi su di una rinnovata tendenza volta da un lato ad utilizzare il contratto collettivo aziendale quale veicolo di regole prevenzionistiche, riconducibili in un certo senso a buone prassi, dall'altro ad incrementare ed avvalorare la partecipazione degli stessi creditori di tutela, in tal modo coinvolti, anche attraverso i loro rappresentanti, nel miglioramento della salute e sicurezza nell'ambiente di lavoro ${ }^{2}$.

L'attenzione deve, quindi, essere posta sul ruolo che, da questo punto di vista, contrattazione e partecipazione stanno assumendo, volgendo, inoltre, lo sguardo all'esperienza di altri ordinamenti nazionali, da tempo maggiormente sensibili a questa problematica (quanto al profilo della partecipazione in chiave comparata cfr. il capitolo III).

È necessario, tuttavia, fin d'ora precisare l'esatto significato delle due espressioni e valutarne le differenze, nonché le possibili «Sovrapposizioni» ${ }^{3}$.

È nota la tradizionale «contrapposizione concettuale» ${ }^{4}$ esistente tra contrattazione e partecipazione, la quale ultima evoca l'idea dell'integrazione, in contrasto con quella del conflitto.

Il sistema di relazioni sindacali in Italia è, infatti, tipicamente

1 C. Smuraglia, Sicurezza e igiene del lavoro. Quadro normativo. Esperienze attuative e prospettive, in RGL, 2001, I, p. 487.

2 Si deve ricordare, in proposito, la contrattazione aziendale di fine anni sessanta e primi anni settanta, i cui ampi contenuti concernevano «diritti di informazione, esami congiunti e commissioni paritetiche, organizzazione del lavoro, inquadramenti, tempo di lavoro, ambiente di lavoro»: cfr. A. LASSANDARI, La contrattazione e il contratto collettivo, in P.G. Alleva, G. Ghezzi (a cura di), Il Diritto del lavoro, Roma, Ediesse, 2002, fasc. 12, p. 17; per un approfondito studio sul contratto collettivo aziendale cfr. ID., Il contratto collettivo aziendale e decentrato, Milano, Giuffrè, 2001.

$3 \mathrm{~V}$. le riflessioni di A. Alaimo, L'eterno ritorno della partecipazione: il coinvolgimento dei lavoratori al tempo delle nuove regole sindacali, in DLM, 2014, p. 313 ss.

4 Cfr. M. D’Antona, Partecipazione, codeterminazione, contrattazione (temi per un diritto sindacale possibile), in RGL, 1992, p. 138 ss. e in B. Caruso, S. Sciarra (a cura di), Massimo D'Antona Opere. Scritti sul diritto sindacale, Milano, Giuffrè, 2000, vol. II, p. 314 ss. 
contraddistinto dalla conflittualità e contrapposizione nella regolazione contrattuale, nonché dall' «esclusività» dell'azione rappresentativa propria delle organizzazioni sindacali ${ }^{5}$. Stenta, invece, ad attecchire, almeno nella generalità delle realtà aziendali, la diversa via della comunanza e condivisione di interessi, che reca inevitabilmente con sé un contenimento dell'autonomia delle parti.

La prospettiva in esame è quella del riconoscimento della partecipazione dei lavoratori nell'ambito della contrattazione collettiva che, come sottolineato, «rappresenta una tappa sulla via del progresso delle forme di democrazia industriale ${ }^{6}$. Vero è che nonostante l'antagonismo che separa i termini menzionati, non si può necessariamente escludere la loro compresenza e convivenza come, appunto, i recenti interventi della fonte negoziale a livello aziendale dimostrano, almeno nell'ambito della tutela del benessere psicofisico dei prestatori di lavoro. Si tratterebbe, allora, di «mezzi diversi e complementari», volti a perseguire «soluzioni comuni» ${ }^{7}$.

Del resto, il tema della salute nell'ambiente lavorativo è, per sua natura, legato alla diffusione di forme partecipative, posto che il «consenso», che si estrinseca nella consultazione dei lavoratori e/o delle loro rappresentanze, è l'«idea-guida», accanto all'«effettività»

5 M. D’Antona, Partecipazione, codeterminazione, contrattazione (temi per un diritto sindacale possibile), cit., p. 137; nonché G. ProiA, La partecipazione dei lavoratori tra realtà e prospettive. Analisi della normativa interna, in DRI, 2010, p. 60 ss. Al riguardo v., inoltre, I. Regalia, Riflettendo ancora sulla partecipazione diretta, in L'impresa al plurale. Quaderni della partecipazione, 2000, p. 15, secondo cui il termine "partecipazione» è divenuto «uno di quelli più diffusi e fungibili, usato e abusato per alludere con valutazioni di segno positivo a quasi tutto ciò che si vuol contrapporre alle vecchie e per lo più screditate prassi di relazioni industriali acquisitivo-conflittuali».

6 E. GHera, Partecipazione dei lavoratori e contrattazione collettiva nell'impresa, in Impresa e Società, 1977, n. 5, p. 8, secondo il quale «tra contrattazione collettiva e partecipazione vi è non antitesi di fini ma eterogeneità di piani d'intervento: la contrattazione è infatti un metodo mentre la partecipazione è un obiettivo dell'azione collettiva dei lavoratori».

7 A. Apostoli, La forza propulsiva dell'art. 46 della Costituzione al di là della sua sostanziale inattuazione, in C. Zol (a cura di), Lavoro e impresa: la partecipazione dei lavoratori e le sue forme nel diritto italiano e comparato, Torino, Giappichelli, 2015, p. 16 ss. 
delle tutele, per perseguire il fine ultimo della prevenzione ${ }^{8}$. È però altrettanto vero che tali manifestazioni sono deboli rispetto al significato che deve riconoscersi al termine partecipazione, in particolare se si riflette sulle peculiarità proprie del sistema tedesco ${ }^{9}$.

Anzitutto, la partecipazione, come chiarito, rappresenta qualcosa di altro e di ulteriore rispetto al «binomio informazione/consultazione», intese come mere situazioni «preparatorie o strumentali» al reale concorso nella creazione di decisioni comuni, e si contraddistingue, essenzialmente, per il necessario raggiungimento di una regola condivisa tra le parti contrapposte ${ }^{10}$.

Il complesso tema della partecipazione dei lavoratori, nelle sue varie raffigurazioni, non è nuovo nel nostro ordinamento e periodicamente s'impone all'attenzione degli studiosi, nonché del legislatore, senza però mai trovare la sua compiuta e definitiva collocazione; da sempre, inoltre, si interseca con il profilo della contrattazione collettiva, attraverso la quale, per così dire, prende forma.

La norma chiave su cui il dibattito si è a lungo incentrato è l'art. 46 della Carta costituzionale, variamente interpretato dalla dottrina, eppure ancora privo di una attuazione legislativa ${ }^{11}$. In merito a tale disposizione «ad efficacia differita», ossia «bisognosa di essere integrata mediante apposite norme strumentali» ${ }^{12}$, non si è mancato di affermare che «il motivo della partecipazione alla gestione e della condecisione non ha avuto fortuna in Italia», non essendosi in linea di principio avverato il superamento della contrapposizione de-

8 L. Montuschi, I principi generali del d.l.gs. n. 626 del 1994 (e le successive modifiche), in ID. (a cura di), Ambiente, salute e sicurezza. Per una gestione integrata dei rischi da lavoro, Torino, Giappichelli, 1997, p. 48.

9 Sul tema si rinvia al capitolo III, paragrafi 5 e 5.1 .

10 M. Pedrazzoli, La partecipazione dei lavoratori: un tema di natura costituzionale e politica, in C. ZOLI (a cura di), Lavoro e impresa: la partecipazione dei lavoratori e le sue forme nel diritto italiano e comparato, cit., p. 2 ss.; nonché ID., Partecipazione, costituzione economica e art. 46 della Costituzione. Chiose e distinzioni sul declino di un'idea, in RIDL, 2005, I, p. 438 ss.

11 In particolare cfr. G. GHezzi, Art. 46, in G. Branca (a cura di), Commentario della Costituzione. Rapporti economici, Bologna-Roma, Zanichelli-Il Foro italiano, 1980, tomo III, p. 69 ss.

12 G.F. MAncinI, La responsabilità contrattuale del prestatore di lavoro, Milano, Giuffrè, 1957, p. 125. 
gli interessi tra imprenditore e lavoratori, né realizzato un interesse comune ${ }^{13}$.

L'attenzione è stata posta sulla «comunità d'impresa, qualificata appunto dalla presenza di un interesse partecipato dall'imprenditore e dal personale» ${ }^{14}$. Si è, tuttavia, anche sostenuto che «l'attribuzione ai lavoratori di un ruolo attivo nella formulazione della politica d'impresa non presuppone né comporta, necessariamente, il riconoscimento di una comunità di interessi munita di una qualificazione autonoma e distinta dagli interessi di cui sono titolari le due parti del contratto di lavoro» ${ }^{15}$.

Da una ulteriore prospettiva «la portata e le potenzialità» ${ }^{16}$ dell'art. 46 Cost. sono state colte ricercando il senso che risulta dalla comparazione con altre norme costituzionali ed in particolare con l'art. 3, comma 2, e 41, comma 3, Cost., nonché dal complesso della Carta costituzionale; è, così, possibile «per il giurista affrontare in modo realistico alcuni temi essenziali», quali quelli dei livelli, dell'oggetto e del metodo della partecipazione ${ }^{17}$, che, anche nel più

13 F. Santoro Passarelli, Il sindacato nell'impresa, in RDL, 1976, I, p. 11 ss., il quale ha sottolineato il consolidarsi della conflittualità negli anni precedenti lo Statuto dei lavoratori e la configurazione nello Statuto della «presenza del sindacato nell'impresa come contrapposta a quella dell'imprenditore»; le rappresentanze sindacali aziendali non sono, infatti, state concepite come «investite di un potere di partecipazione alla gestione dell'impresa», bensì di un «potere distinto e di controllo» rispetto al potere imprenditoriale. In proposito cfr., in particolare, F. GALGANO, L'imprenditore, Bologna, Zanichelli, 1974, p. 109, che ha chiarito come non possa prescindersi dal fatto che "gli interessi dell'imprenditore e dei lavoratori sono interessi fra loro antagonisti»; nonché L. MENGonI, Recenti mutamenti nella struttura e nella gerarchia dell'impresa, in Diritto e valori, Bologna, Il Mulino, 1985, p. 356 ss.; ID., Democrazia industriale: conflitto o partecipazione?, in Mondoperaio, 1983, nn. 7-8, p. 25 ss.

14 G.F. MANCINI, La responsabilità contrattuale del prestatore di lavoro, cit., p. 126.

15 G. Giugni, Mansioni e qualifica nel rapporto di lavoro, Napoli, Jovene, 1963, p. 304 ss.

16 G. GHezzI, La partecipazione dei lavoratori alla gestione delle imprese: il problema di una nuova "lettura" dell'art. 46 della Costituzione, in C. SMuraglia, C. Assanti, F. Galgano, G. Ghezzi, La democrazia industriale. Il caso italiano, Roma, Editori Riuniti, 1980, p. 98 ss.

17 G. GHEzzI, La partecipazione dei lavoratori alla gestione delle imprese: il problema di una nuova "lettura" dell'art. 46 della Costituzione, cit., p. 100; nonché ID., Art. 46, in G. Branca (a cura di), Commentario della Costituzione. Rapporti economici, cit., p. 69 ss. 
recente dibattito, continua ad affascinare gli studiosi, pur nella consapevolezza del suo non materializzarsi, se non occasionalmente e per brevi periodi ${ }^{18}$.

Per comprendere l'art. 46 Cost. è stato rimarcato il raffronto con la definizione di democrazia industriale, intesa come «formazione e costruzione di poteri controbilancianti il management», cui consegue «l'influenza dei lavoratori e delle loro organizzazioni sulla conduzione dell'impresa» ${ }^{19}$.

$\mathrm{Al}$ di là dell'ambiguità del termine partecipazione, al fine del presente studio ci si riferisce in particolare all'istituzione di mezzi, procedure o organismi (bilaterali o a composizione mista), che valorizzano il coinvolgimento dei lavoratori e delle loro rappresentanze nelle decisioni dell'impresa. Da questo punto di vista, diverse sono state, soprattutto, nell'ultimo periodo, le proposte di attuazione della norma costituzionale ${ }^{20}$.

18 In particolare v. L. Zoppoli, Rappresentanza collettiva dei lavoratori e diritti di partecipazione alla gestione delle imprese, in DLRI, 2005, p. 374, secondo il quale il tema della partecipazione è «al contempo intramontabile nella riflessione teorica e pressoché sconosciuto nella prassi»; nonché ID., Modelli partecipativi e tecniche di regolazione dei rapporti di lavoro, in DRI, 2010, 1, p. 19 ss. Sulle «prospettive» di fronte alla «sfida della partecipazione» cfr. F. CARINCI, La partecipazione dei lavoratori alla gestione: la via italiana, in C. AssanTI (a cura di), Dallo Statuto dei diritti dei lavoratori alla dimensione sociale europea, Milano, Giuffrè, 1991, p. 225.

19 M. Pedrazzoli, voce Democrazia industriale, in Digesto delle Discipline Privatistiche, Sezione Commerciale, Torino, Utet, 1989, p. 245. Sul termine «polisenso» di democrazia industriale cfr. B. Veneziani, Stato e autonomia collettiva. Diritto sindacale italiano e comparato, Bari, Cacucci, 1992, p. 268 ss. Sul tema v. Lord Wedderburn of Charlton, G. Giugni, E. Ghera, G.F. Mancini, U. RomagnoLi, G. Minervini, B. Trentin, Democrazia politica e democrazia industriale. Cogestione, partecipazione e controllo in Italia e in Europa, Bari, De Donato, 1978 e, in particolare, G.F. MANCINI, ivi, p. 117, secondo il quale «ogni paese in tema di democrazia industriale» deve seguire la strada «più omogenea alle caratteristiche del sistema di relazioni industriali e più in generale del sistema socio-culturale e politico».

20 Per una riflessione in proposito si veda M. NAPOLI, La discussione parlamentare sulla partecipazione dei lavoratori alla gestione delle imprese: la via del sostegno tributario, in DRI, 2010, p. 72 ss.; ID., Per un progetto legislativo sulla partecipazione, in L'impresa al plurale. Quaderni della partecipazione, 2002, p. 307; G. Prola, La partecipazione dei lavoratori tra realtà e prospettive. Analisi della normativa interna, cit., p. 60 ss.; T. TREU, Le proposte parlamentari sulla partecipazione, in DRI, 2010, p. 93 ss., che ha sottolineato come «i motivi della mancata attuazione costituzionale in tema di partecipazione sono radicati nella nostra storia economica e politico-sociale»; P. Oliveldi, G. Ciocca, La partecipazione del sindacato in generale, in 
Assume, anzitutto, rilievo la 1. n. 92/2012, che all'art. 1, comma 1 , lett. $h$, ha promosso «modalità partecipative di relazioni industriali, in conformità agli indirizzi assunti in sede europea, al fine di migliorare il processo competitivo delle imprese»; la delega ivi contenuta, rimasta inattuata, contemplava all'art. 4, comma 62, lett. c, fra i principi e criteri direttivi, «l'istituzione di organismi congiunti, paritetici o comunque misti, dotati di competenze di controllo e partecipazione nella gestione di materie quali la sicurezza dei luoghi di lavoro e la salute dei lavoratori» ${ }^{21}$.

Ancorché solo sulla carta si è, dunque, riconosciuta la rilevanza dello strumento partecipativo, inscindibilmente connesso al contesto nel quale si inserisce e finalizzato «a favorire una gestione equilibrata sia delle vicende aziendali sia delle relazioni industriali», proprio in virtù del coinvolgimento dei lavoratori e dei loro rappresentanti nel perseguimento della promozione della produttività dell'impresa e della qualità del lavoro ${ }^{22}$.

F. LunARdon (a cura di), Conflitto, concertazione e partecipazione, Padova, Cedam, 2011, p. 773 ss.; P. Ichino, La partecipazione dei lavoratori nell'impresa. Le ragioni di un ritardo, in RIDL, 2013, I, p. 861 ss.; C. Zoli, La partecipazione dei lavoratori alla gestione delle imprese nella recente progettazione legislativa, in ID. (a cura di), Lavoro e impresa: la partecipazione dei lavoratori e le sue forme nel diritto italiano e comparato, cit., p. 112 ss. Si veda, inoltre, la Proposta di legge della Rivista Diritti, Lavori, Mercati, contenente la «Disciplina delle relazioni sindacali, della contrattazione collettiva e della partecipazione dei lavoratori», in DLM, 2014, I, p. 155 ss. e in L. Zoppoli, A. Zoppoli, M. Delfino (a cura di), Una nuova Costituzione per il sistema di relazioni sindacali?, Napoli, Editoriale Scientifica, 2014, p. 539 ss.; nonché la «Carta dei diritti universali del lavoro. Nuovo statuto di tutte le lavoratrici e di tutti $i$ lavoratori» proposta dalla Cgil nel gennaio 2016.

21 In senso critico sull'oggetto della delega contenuta nella 1. n. 92/2012 cfr. M. Pedrazzoli, La partecipazione dei lavoratori: un tema di natura costituzionale e politica, cit., p. 1; nonché L. MARIUCCI, È proprio un very bad text? Note critiche sulla riforma Monti-Fornero, in LD, 2012, n. 3-4, p. 433 ss. In proposito v. M. Rusciano, Contrattazione e sindacato nel diritto del lavoro dopo la l. 28 giugno 2012, n. 92, in Studi in memoria di Mario Giovanni Garofalo, II, Bari, Cacucci, 2015, p. 869 , secondo cui «è ad un sindacato partecipativo che il legislatore guarda, con un chiaro rinvio a modelli e obiettivi invalsi in tutt'altri contesti europei»e «del tutto estranei alla cultura e alla prassi delle nostre relazioni sindacali»; cfr., inoltre, le perplessità sollevate da F. GUARRIELlo, I diritti di informazione e partecipazione, in M. Cinelli, G. Ferraro, O. Mazzotta (a cura di), Il nuovo mercato del lavoro, dalla riforma Fornero alla legge di stabilità 2013, Torino, Giappichelli, p. 755.

22 T. TReu, Flessibilità e tutele nella riforma del lavoro, in WP C.S.D.L.E. “Massimo D’Antona”.IT-155/2012, p. 19 ss., il quale precisa che «l'importanza 
Occorre, altresì, sottolineare che il veicolo, di recente, prescelto da parte dello stesso legislatore per la promozione della salute in senso lato nell'ambiente di lavoro ${ }^{23}$ è proprio la contrattazione collettiva a livello d'azienda. Così ai sensi dell'art. 25, d.lgs. n. 80/2015, in tema di «Misure per la conciliazione delle esigenze di cura, di vita e di lavoro», una quota delle risorse del Fondo per il finanziamento di sgravi contributivi per incentivare la contrattazione di secondo livello è destinata, in via sperimentale, alla promozione della conciliazione tra vita professionale e vita privata.

Nuovi incentivi per la contrattazione aziendale sono stati, inoltre, previsti nella c.d. legge di stabilità del dicembre 2015²4; la scelta di attribuire al contratto collettivo il ruolo di «validare il coinvolgi-

del metodo partecipativo è confermata dalle esperienze dei paesi europei che l'hanno da più tempo praticato, non solo nella forma della presenza di rappresentanti dei lavoratori negli organi delle società, ma come criterio ispiratore della regolazione e della gestione dei rapporti individuali e collettivi di lavoro». Per una riflessione sugli assetti delle relazioni industriali in Europa cfr. M. BIAGI, Cultura e istituti partecipativi delle relazioni industriali in Europa, in L'impresa al plurale. Quaderni della partecipazione, 2002, p. 291. Quanto al rilievo della «partecipazione» quale «pilastro» per un «nuovo sistema di relazioni industriali» cfr., in particolare, l'Accordo Cgil, Cisl, Uil, 14 gennaio 2016, «Un moderno sistema di relazioni industriali. Per un modello di sviluppo fondato sull'innovazione e la qualità del lavoro»; nonché l’Accordo Confapi, Cgil, Cisl, Uil, 26 luglio 2016.

23 In ordine al concetto di «ambiente di lavoro», quale «nozione polisenso che s'è caricata di molteplici profili» cfr. P. Tuluini, Sicurezza sul lavoro e modello sociale europeo: un'ipotesi di sviluppo, cit., p. 1268 ss.

24 Si veda la legge del 28 dicembre 2015, n. 208, «Disposizioni per la formazione del bilancio annuale e pluriennale dello Stato», e in particolare l'art. 1, comma 189, ove è promosso il «coinvolgimento paritetico» dei lavoratori nell'organizzazione del lavoro e l'art. 1, comma 190, lett. a, che ha introdotto incentivi fiscali per il sostegno della contrattazione collettiva. V., altresì, la legge 11 dicembre 2016, n. 232, «Bilancio di previsione dello Stato per l'anno finanziario 2017 e bilancio pluriennale per il triennio 2017-2019», che all'art. 1, commi 160-162, ha apportato modifiche alla 1. n. 208/2015; nonché il Decreto interministeriale del 25 marzo 2016, ove sono stabilite le modalità attuative delle previsioni contenute nell'art. 1, commi da 182 a 191, 1. n. 208/2015 e l'Accordo interconfederale del 14 luglio 2016, con il quale si intende perseguire gli obiettivi definiti nella citata legge n. 208/2015, confermando «l'opportunità della progressiva valorizzazione della contrattazione di secondo livello». Sul tema cfr. in particolare le considerazioni di S. MaInARDi, Le relazioni collettive nel "nuovo" diritto del lavoro, in Legge e contrattazione collettiva nel diritto del lavoro post-statutario, Atti delle giornate di studio di diritto del lavoro, Napoli, 16-17 giugno 2016, Milano, Giuffrè, 2017, p. 256 ss.; cfr., altresì T. TReu, Introduzione Welfare aziendale, in WP C.S.D.L.E. "Massimo D'Antona” .IT-297/2016; B. CARUso, "The bright side of the moon": politiche 
mento dei lavoratori nell'organizzazione del lavoro» ha, tuttavia, suscitato alcune perplessità in dottrina, inducendo a parlare di «contrattazione addomesticata» piuttosto che di «partecipazione» ${ }^{25}$.

In ogni caso, il «terreno» sembra ora «più propizio» per ripensare la rilevanza e la collocazione del coinvolgimento dei prestatori di lavoro nel sistema delle relazioni industriali, «senza che vengano abbandonate le tecniche tradizionali incentrate sulla contrattazione collettiva» ${ }^{26}$; ciò anche e soprattutto per gli aspetti della salute nel lavoro.

2. La promozione della salute nel lavoro attraverso la contrattazione collettiva aziendale

Anche in ragione dell'attuale assenza di un quadro normativo che regolamenti gli aspetti partecipativi fin qui considerati, assumono sempre più significato i contenuti della fonte collettiva, seppur numericamente marginali e non rappresentativi della generalità delle realtà aziendali. Non è, infatti, preclusa alla contrattazione collettiva la possibilità di realizzare modalità e procedure di coinvolgimento dei lavoratori, al di là del richiamo alla legge contenuto nel menzionato art. 46 Cost. $^{27}$.

A diversi anni dalle prime riflessioni svolte in merito alle linee di sviluppo della contrattazione collettiva in tema di tutela della sicurezza dei prestatori di lavoro, è opportuno ora tentare di tracciare un bilancio. Allora si definì tale esperienza, contraddistinta da «ombre» senza dubbio prevalenti rispetto ai «pochi e rari spiragli di luce», in termini di assoluta delusione ${ }^{28}$.

del lavoro personalizzate e promozione del welfare occupazionale, in RIDL, 2016, n. 2, p. 199.

25 D. GotTARDI, La contrattazione collettiva tra destrutturazione e ri-regolazione, in $L D, 2016$, n. 4 , p. 907 , la quale si riferisce a «territori negoziali convenienti che consentono l'alleggerimento del costo del lavoro».

26 C. Zoli, La partecipazione dei lavoratori alla gestione delle imprese nella recente progettazione legislativa, in ID. (a cura di), Lavoro e impresa: la partecipazione dei lavoratori e le sue forme nel diritto italiano e comparato, cit., p. 110.

27 P. Olivelli, La partecipazione dei lavoratori tra diritto comunitario e ordinamento italiano, in DRI, 2010, p. 50.

28 L. Montuschi, Diritto alla salute e organizzazione del lavoro, cit., p. 165 ss. 
Note sono le difficoltà generalmente mostrate in passato dall'autonomia collettiva nel trattare la materia della tutela della salute dei prestatori di lavoro. In particolare la contrattazione collettiva nazionale ha preferito privilegiare la protezione del posto di lavoro e del salario, anche in ragione di situazioni di crisi economica, trascurando la tutela del diritto fondamentale e primario alla salute sul lavoro, divenuto oggetto di «formule di stile destinate a passare inalterate da un rinnovo negoziale all'altro» ${ }^{29}$.

Ancora molto attuale è l'interrogativo che concerne la possibilità o meno di contrattare regole in materia di protezione dei lavoratori. La sicurezza sul lavoro, per sua natura non negoziabile da parte dello strumento contrattuale, può essere negoziata?

Rispetto al periodo prestatutario, sono state criticamente poste in luce le clausole contrattuali di monetizzazione della salute, intese non a rimuovere i rischi bensì ad attribuire al lavoratore indennità legate alla pericolosità dell'ambiente lavorativo, contrariamente «ad ogni principio etico e giuridico»: il bene salute, infatti, in quanto inalienabile, deve essere sottratto allo scambio ${ }^{30}$. La «linea della monetizzazione» è stata, invero, abbandonata alla fine degli anni sessanta fino ai primi anni settanta, contraddistinti da un forte impegno delle parti sociali su questi temi, con il riconoscimento alle rappresentanze sindacali aziendali del diritto di partecipare alla ricerca ed adozione delle misure di prevenzione: ne scaturì «l'apertura di un dialogo» e la nascita di una «coscienza collettiva» intorno all'organizzazione del lavoro ${ }^{31}$.

29 L. Montuschi, L'incerto cammino della sicurezza del lavoro fra esigenze di tutela, onerosità e disordine normativo, in RGL, 2001, I, p. 513.

30 L. Montuschi, La tutela della salute e la normativa comunitaria: l'esperienza italiana, in M. BIAGI (a cura di), Tutela dell'ambiente di lavoro e direttive Cee, Rimini, Maggioli, 1991, p. 13 ss. In proposito cfr., inoltre, G.G. BaLANDI, Individuale e collettivo nella tutela della salute nei luoghi di lavoro: l'art. 9 dello statuto, in $L D, 1990$, n. 2, p. 221, che sottolinea come «la collocazione dell'obbligo di sicurezza nel cuore del rapporto di lavoro» rafforza l'affermazione secondo la quale «si tratta di una obbligazione non negoziabile».

31 Cfr. L. Montuschi, Diritto alla salute e organizzazione del lavoro, cit., p. 178 e i riferimenti ivi contenuti. Sul tema cfr., altresì, S. RENGA, Modello sindacale di tutela della salute nei luoghi di lavoro dal dopoguerra agli anni novanta, in LD, 1994, n. 4, p. 622; M. Ricci, Sicurezza sul lavoro: controllo e partecipazione sindacale tra iure condito $e$ de iure condendo, in Scritti in onore di Edoardo Ghera, cit., p. 987. 
Tuttavia, nel successivo periodo di crisi economica, compreso tra la fine degli anni settanta e la metà degli anni ottanta, l'attenzione si è focalizzata altrove, con il conseguente regredire della «linea strategica della salute» ${ }^{32}$.

Ora la situazione su cui si riflette appare, nuovamente, diversa: attraverso il negozio si contrattano, infatti, misure volte a migliorare l'ambiente di lavoro e indirizzate al benessere in senso lato dei prestatori di lavoro. Quindi, se è vero che nessun compromesso è consentito nella materia de qua, il discorso cambia, almeno astrattamente, ove vengano in gioco strumenti ulteriori, non espressamente contemplati dalla fonte legislativa.

Non v'è dubbio che il rischio insito nel lavoro non possa essere monetizzato e le considerazioni formulate tempo addietro siano quanto mai attuali. Occorre, tuttavia, valutare l'odierno ruolo svolto dall'autonomia collettiva, tenendo separato il livello nazionale da quello aziendale.

Si può anticipare, fin da subito, che la contrattazione collettiva nazionale presenta ancora un rilievo alquanto marginale, al contrario dei recenti scenari propri della fonte negoziale a livello aziendale.

Gli interventi della contrattazione collettiva nazionale, per lo più in esecuzione dei rinvii contenuti nel d.lgs. n. 81/2008, continuano, infatti, ad essere scarni ${ }^{33}$; si tratta fondamentalmente di previsioni contrattuali poco rilevanti ed incisive rispetto all'essenza dell'obbligo di sicurezza.

Il benessere dei prestatori di lavoro è, invece, sempre più tra $\mathrm{i}$ contenuti della regolamentazione collettiva aziendale, la quale sta

32 L. Montuschi, La tutela della salute e la normativa comunitaria: l'esperienza italiana, cit., p. 14.

33 Il d.lgs. n. 81/2008 rinvia di rado all'autonomia collettiva, in particolare in ordine alle modalità di designazione o di elezione del rappresentante dei lavoratori per la sicurezza (anche territoriale e di sito produttivo), di svolgimento delle relative funzioni e di determinazione dei contenuti specifici della sua formazione (cfr. gli artt. 37, 47-50); ulteriore rinvio alla contrattazione collettiva, anche aziendale, è contenuto, ad esempio, nell'art. 175, d.lgs. n. 81/2008, relativamente all'individuazione delle modalità delle interruzioni delle attività per gli addetti ai videoterminali. Per un commento v. G. Natullo, Il quadro normativo dal Codice civile al Codice della sicurezza sul lavoro. Dalla Massima Sicurezza possibile alla Massima Sicurezza effettivamente applicata?, in ID. (a cura di), Salute e sicurezza sul lavoro, cit., p. 39. 
assumendo la tendenza ad introdurre regole aggiuntive e migliorative dell'ambiente di lavoro; la materia, per quanto non adatta ad essere negoziata, è attratta nell'area della contrattazione collettiva ${ }^{34}$. Il livello aziendale si sta, così, mostrando particolarmente adatto a suggerire e incentivare nuove forme di tutela, mirate a diminuire i rischi di infortuni e malattie professionali.

Vero è che, anche con riferimento ad altri ordinamenti nazionali, la tutela collettiva della salute dei prestatori di lavoro assume peculiare importanza ${ }^{35}$.

Rinvii alla contrattazione di secondo livello sono presenti nella stessa fonte negoziale nazionale, ove si conviene di affidare al livello aziendale la programmazione degli interventi da attuare nell'ambiente di lavoro ${ }^{36}$; sono, infatti, espressamente valorizzate le «soluzioni negoziali che tengano conto della dimensione delle imprese» ${ }^{37}$.

La contrattazione collettiva aziendale tende a prendere in considerazione, specificando e a volte ampliando, il contenuto dell'obbligo di sicurezza come individuato nel d.lgs. n. 81/2008; tende, in sostanza, a "contrattualizzare" metodi e prassi, con il fine dichiarato di garantire la sicurezza e il benessere dei prestatori di lavoro attra-

34 A favore di un «atteggiamento non negativo» nei confronti della contrattazione collettiva in materia v. G.G. BALANDI, Individuale e collettivo nella tutela della salute nei luoghi di lavoro: l'art. 9 dello statuto, cit., p. 228 ss., il quale ne sottolinea l'«opportunità» e insieme il «rischio»; sulla possibile «convivenza» in una «posizione di complementarietà» tra partecipazione del Rls e contrattazione aziendale della Rsu v. P. Campanella, Profili collettivi di tutela della salute e rappresentanza dei lavoratori per la sicurezza: disciplina legislativa, bilancio applicativo, prospettive di riforma, in $R G L, 2007$, supplemento al n. 2, p. 177. In ordine alla «dimensione collettiva» della sicurezza sul lavoro v., altresì, P. PAscucci, Dopo la legge n. 123 del 2007. Prime osservazioni sul Titolo I del d.lgs. 9 aprile 2008, n. 81 in materia di tutela della salute e della sicurezza nei luoghi di lavoro, cit., p. 152 ss.; P. TulLINI, Sicurezza sul lavoro e modello sociale europeo: un'ipotesi di sviluppo, cit., p. 1271 ss.; M. LAI, La sicurezza del lavoro tra legge e contrattazione collettiva, Torino, Giappichelli, 2002, p. 94 ss.

35 Quanto al ruolo della contrattazione collettiva in materia nell'ordinamento spagnolo cfr. il paragrafo 4.

36 Si veda, ad esempio, l'art. 6 del CCNL del 6 novembre 2014, per artisti, tecnici, amministrativi e ausiliari dipendenti da società cooperative e imprese sociali operanti nel settore della produzione culturale e dello spettacolo.

37 Cfr. l'Accordo di rinnovo CCNL del 15 ottobre 2015, per gli addetti all'industria chimica, chimico-farmaceutica, delle fibre chimiche e dei settori abrasivi, lubrificanti e gpl. 
verso un loro più accentuato coinvolgimento. La più recente negoziazione a livello d'azienda, pur rimanendo di nicchia, offre interessanti spunti di riflessione.

Nelle disposizioni contrattuali, concernenti in prevalenza le realtà di grandi dimensioni, è rimarcato l'impegno a «mantenere relazioni sindacali che contribuiscano al raggiungimento degli obiettivi aziendali in un'ottica di attenzione ai lavoratori», così da garantire «una più efficace gestione» delle problematiche dell'organizzazione del lavoro, della salute e sicurezza dell'ambiente di lavoro, della formazione e dello sviluppo professionale ${ }^{38}$.

Come precisato, «gli aspetti collegati alla sicurezza delle persone e dei luoghi di lavoro rappresentano un elemento centrale e prioritario» nella cultura e nell'organizzazione aziendale; acquista, dunque, particolare importanza «un contesto organizzativo idoneo a favorire il coinvolgimento proattivo di tutti gli attori», nonché l'azione dei rappresentanti dei lavoratori per la sicurezza, il cui ruolo è anche quello di «stimolare e suggerire» interventi di miglioramento delle condizioni complessive di sicurezza ${ }^{39}$. L'intento proclamato è quello di applicare standard più rigorosi di quelli richiesti dalla legislazione vigente, attraverso «un idoneo e costante dialogo» ${ }^{40}$.

Al fine di raggiungere l'obiettivo «zero infortuni», l'attenzione è in particolare rivolta all'arricchimento delle forme di formazione, informazione e, soprattutto, di coinvolgimento dei lavoratori e/o loro rappresentanze ${ }^{41}$. In tal senso, è promossa la realizzazione di

38 In tal senso cfr. il contratto collettivo aziendale Linde Gas Italia S.r.l. e Linde Medicale S.r.l. del 20 giugno 2017, teso a favorire un «percorso virtuoso» di «miglioramento continuo delle performance aziendali e delle condizioni di lavoro».

39 Cfr. il contratto collettivo aziendale Barilla G. e R. Fratelli S.p.a. del 12 novembre 2014; cfr., inoltre, il contratto collettivo aziendale Spumador S.p.a. del 24 febbraio 2017, ove è affidato al RLS il compito di presentare ogni anno dettagliatamente le proposte di interventi in materia di sicurezza, igiene e prevenzione, che saranno valutate dalla Direzione aziendale «anche in relazione al rapporto costi/benefici», promuovendo occasioni di incontro al fine di «definire le priorità, i tempi e le modalità degli interventi ritenuti necessari».

40 Così il contratto collettivo aziendale Bolton Alimentari S.p.a. del 15 luglio 2014, ove è ribadito l'impegno costante per il raggiungimento e mantenimento di «obiettivi di sicurezza» superiori a quelli legislativamente previsti; in termini simili v. il contratto collettivo aziendale del gruppo Ferrero del 25 luglio 2014.

41 Così il citato contratto collettivo aziendale Barilla G. e R. Fratelli S.p.a. del 12 novembre 2014. Cfr., inoltre, il contratto collettivo aziendale Linde Gas Italia 
«iniziative finalizzate a sensibilizzare i collaboratori ad uno stile di vita sostenibile sotto il profilo della salute, della sicurezza e dell'ambiente», nonché la «previsione di riunioni quotidiane, settimanali e mensili», con il precipuo scopo di individuare «azioni e prassi migliorative» ${ }^{42}$.

Esempi di relazioni sindacali e partecipative sono rinvenibili in accordi di grandi gruppi industriali, come quello Volkswagen, e ricordano il sistema di Electrolux-Zanussi, che rappresenta «l'esperienza compiuta più avanzata di democrazia industriale nel nostro Paese» ossia «il punto più alto» in materia di partecipazione dei lavoratori ${ }^{43}$.

I recenti contratti integrativi «di partecipazione» aziendale di Volkswagen Group Italia S.p.a., nonché di Lamborghini e di Ducati, recepiscono i principi contenuti nella «Carta dei rapporti di lavoro per le società e gli stabilimenti del Gruppo», che attribuiscono diritti di «informazione», «consultazione» e «cogestione» in capo alle rappresentanze dei lavoratori in ordine alla «organizzazione del lavoro, sicurezza sul lavoro e tutela della salute» ${ }^{44}$; nell'ottica del migliora-

S.r.l. e Linde Medicale S.r.l. del 20 giugno 2017, cit.

42 Così il contratto collettivo aziendale del gruppo Mondelez del 19 giugno 2014, che sottolinea la «priorità» accordata alla tematica della prevenzione dei rischi. Si veda il contratto integrativo aziendale di Luxottica Group S.p.a. del 30 ottobre 2015, ove sono rimarcate le iniziative intraprese «nell'ottica di continuo miglioramento e innovazione in materia di prevenzione della salute e sicurezza dei lavoratori», nonché gli obiettivi da raggiungere tra i quali lo sviluppo di «una cultura della sicurezza pienamente condivisa», attraverso la partecipazione attiva dei lavoratori e la costruzione di «un ambiente relazionale migliore». In proposito cfr., inoltre, il contratto integrativo aziendale Unipol Gruppo Finanziario S.p.a. del 13 maggio 2016, ove è precisato «l'impegno a promuovere insieme ai rappresentanti dei lavoratori per la sicurezza iniziative rivolte alla tutela della salute dei dipendenti», tra le quali il miglioramento dell'informazione in materia.

43 A. Perulli, Il Testo Unico sul sistema di relazioni sindacali e di partecipazione della Electrolux-Zanussi, in LD, 1999, pp. 41 e 50; ID., Ascesa e declino della partecipazione alla Zanussi Electrolux, in DLM, 2005, p. 619; al riguardo cfr., altresì, L. Mariucci, Per un nuovo rapporto fra conflitto e partecipazione, in LI, 1997, n. 10, p. 5 ss.; A. LASSANDARI, Il contratto collettivo aziendale e decentrato, cit., p. 405.

$44 \mathrm{Si}$ veda la «Carta dei rapporti di lavoro per le società e gli stabilimenti del Gruppo Volkswagen» siglata il 29 ottobre 2009 a Zwickau dal Consiglio di Amministrazione del Gruppo Volkswagen, dal Consiglio di Fabbrica Europeo del Gruppo Volkswagen e Consiglio di Fabbrica Mondiale del Gruppo Volkswagen, dalla Confederazione Sindacale Internazionale dei Metalmeccanici. 
mento delle condizioni di lavoro è, in tal modo, favorita la «cooperazione» e «partecipazione attiva alle decisioni condivise», per la cui «declinazione» e «applicazione» la Carta rinvia alla dimensione del contratto aziendale ${ }^{45}$.

$\mathrm{Si}$ intende, appunto, riflettere sulla promozione di misure integrative rispetto a quelle imposte per via legislativa, dirette al coinvolgimento ed alla fidelizzazione dei lavoratori ${ }^{46}$ all'interno dell'organizzazione aziendale. Assumono rilevanza, da questo punto di vista, gli organismi definiti «congiunti», costituiti da rappresentanti aziendali e da rappresentanti del coordinamento sindacale, introdotti per via contrattuale e diretti alla promozione ed implementazione delle materie oggetto di contrattazione/partecipazione ${ }^{47}$.

Come anticipato, il tema si intreccia con quello complesso della partecipazione dei lavoratori nelle sue molteplici configurazioni, che ha destato l'attenzione del legislatore, poco propenso ad affrontare questa problematica, e rinvigorito il dibattito anche nell'ordinamento italiano ${ }^{48}$.

Con riferimento alla già menzionata delega contenuta nell'art. 4, comma 62, lett. c, 1. n. 92/2012, si deve sottolineare il proposito, rimasto tuttavia inattuato, di regolare i richiamati organismi, secondo l'esempio fornito in altri contesti nazionali.

45 Cfr. il contratto integrativo aziendale Automobili Lamborghini S.p.a. dell'8 giugno 2015; cfr., altresì, il contratto integrativo di partecipazione aziendale Volkswagen Group Italia S.p.a. dell'8 marzo 2016 e il contratto integrativo e di partecipazione Ducati Motor Holding S.p.a. del 4 marzo 2015. Sul tema v. A. LASSANDARI, La contrattazione integrativa, in M. MASCINI (a cura di), L'Annuario del lavoro 2014, Edizioni Il diario del lavoro, Roma, 2014, p. 107.

46 Sul significato e gli strumenti di fidelizzazione cfr. C. Zol, Fidelizzazione del lavoratore, in Lessico giuslavoristico, ordinato da M. Pedrazzoli, Bologna, Bononia University Press, 2010, vol. II, p. 44 ss.

47 Per una riflessione su questi istituti contrattuali v. in particolare S. RENGA, Modello sindacale di tutela della salute nei luoghi di lavoro dal dopoguerra agli anni novanta, cit., p. 617 ss.

48 Cfr. C. Zoli, La partecipazione dei lavoratori alla gestione delle imprese nella recente progettazione legislativa, cit., p. 109 ss.; cfr., inoltre, M. NAPOLI, Partecipazione e fonti del Diritto del lavoro, in Rappresentanza collettiva dei lavoratori e diritti di partecipazione alla gestione delle imprese, Atti delle giornate di studio di diritto del lavoro, Lecce, 27-28 maggio 2005, Milano, Giuffrè, 2006, p. 299, il quale ha sottolineato che il tema della partecipazione si configura come «tema fondamentale e non congiunturale per una prospettiva ricostruttiva dei rapporti di lavoro». 
In assenza di una disciplina legislativa, diversi accordi prevedono l'istituzione di specifiche commissioni tecniche bilaterali dedite ai temi della salute, dell'ergonomia, dell'organizzazione dei tempi e metodi del lavoro, cui sono attribuiti poteri in prevalenza consultivi e propositivi ma non deliberativi, rimanendo la funzione negoziale una «prerogativa del tavolo sindacale aziendale» ${ }^{49}$; l'obiettivo è di analisi, studio ed approfondimento dei problemi e di elaborazione di proposte «attraverso confronti di natura analitico-progettuale e non negoziale ${ }^{50}$. Proposte da cui può scaturire, semmai, una successiva fase negoziale, di competenza delle organizzazioni sindacali.

V'è, pertanto, una netta suddivisione di competenze con le rappresentanze sindacali unitarie, considerati «i limiti» di costituire comitati tecnici dotati di funzioni decisionali in un sistema contraddistinto da un canale unico di rappresentanza ${ }^{51}$. Precisamente la $\mathrm{cd}$. «Commissione Sicurezza» è, spesso, definita come la sede nella quale promuovere la partecipazione, attraverso la formulazione di suggerimenti e richieste di azioni e programmi di miglioramento ${ }^{52}$. Ecco allora che all'interno della stessa commissione possono essere progettate «soluzioni organizzative o procedurali» innovative ${ }^{53}$.

49 Così è specificato nel contratto collettivo aziendale Automobili Lamborghini S.p.a. del 4 luglio 2012, a cui il più recente contratto dell'8 giugno 2015 fa riferimento. Peculiare è, invece, quanto previsto nel «Testo Unico sul sistema di relazioni sindacali e di partecipazione» di Electrolux-Zanussi, sottoscritto il 21 luglio 1997, ove agli organismi congiunti ivi istituiti erano affidate funzioni «deliberanti», con la possibilità di «assumere direttamente decisioni dotate di efficacia esecutiva».

50 In tal senso il citato contratto collettivo aziendale Barilla del 12 novembre 2014. Commissioni tecniche paritetiche sono, altresì, previste nel contratto collettivo aziendale Nestlé del 30 luglio 2014.

51 Cfr. A. Perulli, Intervento, in Rappresentanza collettiva dei lavoratori e diritti di partecipazione alla gestione delle imprese, Atti delle giornate di studio di diritto del lavoro, Lecce, 27-28 maggio 2005, cit., p. 306. In merito alla «forma esclusiva o dualistica del canale rappresentativo» cfr. le riflessioni di M. D'ANToNA, Partecipazione dei lavoratori alla gestione delle imprese, in Enc. Giur. Treccani, vol. XXII, Roma, 1990 e in B. Caruso, S. Sciarra (a cura di), Massimo D'Antona Opere, vol. II, Scritti sul diritto sindacale, cit., p. 216; sul tema delle diverse forme di rappresentanza in chiave comparata cfr. M. BIAGI, Rappresentanza e democrazia in azienda. Profili di diritto sindacale comparato, Rimini, Maggioli, 1990, p. 21 ss.

52 Cfr. al riguardo il contratto collettivo di secondo livello di Metro Italia Cash \& Carry S.p.a. del 29 gennaio 2016.

53 Cfr. la definizione di «buone prassi» contenuta nell'art. 2, comma 1, lett. v, d.lgs. n. 81/2008. 
Tali strumenti designati alla condivisione di misure di tutela della persona del lavoratore, concorrono a individuare un moderno sistema di relazioni industriali, pur non dovendosi confondere il meccanismo partecipativo introdotto dalla fonte collettiva, ancora piuttosto debole, con il differente e complesso modello della codeterminazione aziendale proprio del sistema tedesco, difficilmente impiantabile altrove ${ }^{54}$.

Rispetto alla contrattazione collettiva nazionale dal contenuto piuttosto generico, sempre maggiore rilievo pare, allora, assumere la dimensione aziendale, volta ad esaltare le specificità della singola azienda. Il contratto integrativo, infatti, concorre sempre più a plasmare e «definire l'organizzazione del lavoro e dunque l'organizzazione aziendale $»^{55}$, che è un concetto chiave attorno a cui ruota la protezione della salute del lavoratore ${ }^{56}$. La negoziazione a livello

54 Il tema della partecipazione organizzativa assume notevole importanza nell'Accordo Cgil, Cisl, Uil, del 14 gennaio 2016, cit.; in proposito v. M. TIRABOSCHI, Riforma della contrattazione: la proposta sindacale, in Contratti \& Contrattazione Collettiva, 2016, n. 2, p. 4, secondo cui «dinamica è la contrattazione decentrata in materia di partecipazione organizzativa: il $40 \%$ dei contratti collettivi regola gli istituti dei diritti di informazione e consultazione, mentre il 13\% dei contratti aziendali disciplina i comitati paritetici con funzioni specifiche in materia di formazione, pari opportunità e sicurezza». Si veda, inoltre, l'Accordo Interconfederale tra Confapi, Cgil, Cisl, Uil, del 26 luglio 2016, cit.; nonché le «Linee programmatiche per la crescita della produttività e della competitività in Italia», del 16 novembre 2012, tra Abi, Ania, Confindustria, Alleanza Cooperative, Rete imprese Italia, Cisl, Uil, Ugl; in proposito v. A. Lassandari, Divisione sindacale e "crescita di produttività e competitività", in $L D, 2013$, n. 2, p. 249 ss.

55 R. RomeI, Il contratto collettivo nel nuovo scenario di relazioni industriali, in L. Corazza, R. Romei (a cura di), Diritto del lavoro in trasformazione, Il Mulino, Bologna, 2014, p. 89 ss.; nonché ID., L'autonomia collettiva nella dottrina giuslavoristica: rileggendo Gaetano Vardaro, in DLRI, 2011, p. 209. Cfr., altresì, R. DE Luca Tamajo, Riforme (im)possibili nel diritto sindacale, in DLM, 2011, p. 3, il quale sottolinea come la contrattazione collettiva aziendale appare la «sede privilegiata per coniugare le tutele dei lavoratori e i diritti del sindacato con le specificità organizzative e le esigenze di competitività e produttività delle imprese». In ordine al ruolo del contratto collettivo quale «naturale strumento di regolamentazione della organizzazione del lavoro in azienda» cfr. le considerazioni di A. LASSANDARI, $I l$ contratto collettivo aziendale e decentrato, cit., p. 319 ss.

56 P. Pascucci, Introduzione, in L. Angelini (a cura di) La prevenzione dei rischi da stress lavoro-correlato. Profili normativi e metodiche di valutazione, Atti del Convegno, Urbino, 8 novembre 2013, in I Working Papers di Olympus, n. 31/2014, p. 6. Si veda, inoltre, A. VIScomi, Cresce la partecipazione con le norme sulla sicurezza, in $L I, 1995$, n. 11, p. 15, il quale riconosce che il nesso tra organiz- 
d'impresa sta, così, acquisendo il compito di stabilire rapporti favorevoli tra management e prestatori di lavoro ${ }^{57}$.

Da questo punto di vista, si sta altresì diffondendo la tendenza a promuovere per via contrattuale, attraverso la costituzione di appositi Comitati bilaterali, iniziative volte a migliorare il clima aziendale e interventi di sostegno della qualità dei rapporti all'interno dell'azienda e di contrasto di eventuali situazioni di criticità ${ }^{58}$. Si tratta dei c.d. "accordi di clima", ovvero di "clima organizzativo", il cui fine precipuo è quello di prevenire il rischio stress collegato al lavoro e di perseguire il miglioramento del benessere ed insieme della produttività aziendale ${ }^{59}$.

Tali accordi, idonei ad «instaurare un sistema di partecipazione collettiva alla sicurezza», come precisato, possono incidere positivamente sulla salute in senso lato dei prestatori di lavoro ${ }^{60}$. La «condivisione di scelte organizzative e/o gestionali», congeniale ad

zazione e sicurezza «ha l'effetto di rafforzare sensibilmente il fronte preventivo». Sul concetto di «organizzazione» nel d.lgs. n. 81/2008 cfr. F. CARINCI, Habemus il testo unico per la sicurezza e la salute dei lavoratori: il d.lgs. 9 aprile 2008, n. 81, in $A D L, 2008$, nn. 4-5, p. 986.

57 Sul punto sia consentito rinviare a M. VINCIERI, Attività sindacale e benessere organizzativo dei lavoratori, in DLM, 2016, n. 3, p. 638.

$58 \mathrm{Si}$ veda, in particolare, il contratto collettivo del Gruppo Banco Desio del 24 luglio 2017; il contratto collettivo di secondo livello del Gruppo Intesa San Paolo del 7 ottobre 2015; il contratto integrativo aziendale Benetton Group del 22 gennaio 2015; il contratto integrativo del Gruppo Hera del 24 marzo 2010.

59 Cfr. il Report «Workplace practices: Patterns, performance and wellbeing», Eurofound, 2015, p. 27, ove si chiarisce che «la scarsa motivazione dei lavoratori e il peggioramento del clima lavorativo sono indici di problemi di natura psico-sociale», riguardanti il benessere nell'ambiente di lavoro.

60 P. LAmbertucci, Sicurezza sul lavoro e rischi psico-sociali: profili giuridici, in DLM, 2010, p. 361; nonché, ID., Conclusioni, in L. ANGelini (a cura di), La prevenzione dei rischi da stress lavoro-correlato. Profili normativi e metodiche di valutazione, cit., p. 152, il quale ha precisato come «dovrebbe essere favorita la stipula degli accordi di clima che potrebbero introdurre momenti di "raccordo" tra organizzazione del lavoro e vita personale e familiare»; nonché P. CAMPANELLA, Benessere al lavoro, produttività dell'impresa, partecipazione dei lavoratori in tempo di crisi, ivi, p. 57 ss.; cfr., inoltre, le riflessioni di P. PAscucci, Dopo la legge n. 123 del 2007. Prime osservazioni sul Titolo I del decreto legislativo n. 81 del 2008 in materia di tutela della salute e della sicurezza nei luoghi di lavoro, cit., p. 150, secondo cui «la contrattazione collettiva gioca un ruolo decisivo in materia di salute e sicurezza anche quando non si occupa direttamente di questi temi» ed «affronta tematiche legate all'organizzazione del lavoro». 
«un clima non conflittuale all'interno dell'azienda», permette, inoltre, di raggiungere «più elevati livelli di efficienza e produttività nell'impresa» ${ }^{61}$.

L'impressione è, allora, che la contrattazione collettiva aziendale possa occupare spazi non indifferenti nella disciplina della materia, negoziando soluzioni eticamente accettate e condivise ed integrando su più fronti la legge ${ }^{62}$. Proprio con riferimento alla tutela della salute dei lavoratori, in ossequio ai parametri di cui all'art. 2087 c.c., ed in particolare all'evoluzione della tecnica, si riscontrano interventi propositivi e di sperimentazione di nuove misure protettive.

In conclusione, nonostante il passare del tempo, non pare del tutto cambiata la posizione assunta dalla contrattazione nazionale, mentre per certi versi significativo è il panorama offerto dalla fonte

61 P. LAmbertucci, Sicurezza sul lavoro e rischi psico-sociali: profili giuridici, cit., p. 362. Degno di nota, pur in un'ottica diversa se non contraria, è l'accordo di Pomigliano d'Arco del 15 giugno 2010, incentrato su un nuovo modello di organizzazione del lavoro e, in particolare, su «soluzioni ergonomiche migliorative» basate sul sistema c.d. Ergo-Uas, finalizzato ad un aumento della produzione e ad una riduzione dei costi: al riguardo, in ordine al conseguente «probabile peggioramento delle condizioni materiali di lavoro» e dello stesso «clima organizzativo» cfr. M. Brollo, Uno sguardo d'assieme, in F. CARINCI (a cura di), Da Pomigliano a Mirafiori: la cronaca si fa storia, Milano, Ipsoa, 2011, p. 120 ss.; nonché A. LASSANDARI, Modello organizzativo: il sistema Ergo-Uas, ivi, p. 160 ss., il quale si sofferma sulla compatabilità tra il citato sistema e la normativa in materia di sicurezza sul lavoro. Sul tema cfr., altresì, le osservazioni critiche di L. MenghinI, Lo Statuto dei lavoratori dall'autunno caldo del 1969 all'inverno gelido del 2010-2011, in Studi in onore di Tiziano Treu. Lavoro, istituzioni, cambiamento sociale, I, Il diritto del lavoro e $i$ suoi interlocutori. Diritto sindacale e relazioni industriali, Napoli, Jovene, 2011, p. 328.

62 In ordine agli «interessanti» contenuti dei contratti di secondo livello, che «testimoniano soprattutto della possibilità di coniugare efficacemente gli obiettivi di competitività delle imprese e di tutela delle condizioni di lavoro» cfr. L. BELLARDI, Obiettivi, discipline e buone pratiche dei contratti di secondo livello: una breve rassegna, in L. Montuschi (a cura di), Un diritto in evoluzione. Studi in onore di Yasuo Suwa, cit., p. 736. Al riguardo v. M. Tiraboschi, Contrattazione collettiva: un'analisi empirica del periodo 2012-2014, in Contratti \& Contrattazione Collettiva, 2015, n. 4, p. 5, secondo cui la contrattazione decentrata che dedica al tema grande attenzione è «chiamata a concorrere con le normative nazionali e gli standard internazionali nel tentativo di eliminare, o quantomeno ridurre, gli incidenti, causa di danni alle persone e alle cose». In merito ai «nuovi assetti» della contrattazione collettiva cfr. A. Pizzoferrato, L'autonomia collettiva nel nuovo diritto del lavoro, in DLRI, 2015, 3, p. 411 ss. 
contrattuale a livello d'azienda, incline a perseguire obiettivi di continuo miglioramento del benessere lavorativo.

3. Natura ed efficacia delle misure prevenzionistiche contrattualizzate

Posto che la fonte negoziale sta divenendo, per così dire, veicolo di forme di partecipazione nell'impresa, occorre domandarsi se le menzionate misure a garanzia del benessere dei prestatori di lavoro possano essere considerate esiti del "conflitto" tra le parti e se possa prospettarsi su questo tema una convivenza tra collaborazione e conflitto, tra partecipazione e contrattazione collettiva.

Dunque, verso quale «politica delle relazioni industriali nell'ambiente di lavoro» ${ }^{63} \mathrm{ci}$ si deve orientare?

È stata affermata la «coesistenza pacifica» tra partecipazione e altre «dimensioni dell'azione collettiva», ove il «sistema partecipativo sia visto come una risorsa aggiuntiva e non sostitutiva» rispetto alle dinamiche conflittuali e contrattuali ${ }^{64}$.

Si è, in particolare, delineato il «superamento» della tradizionale contrapposizione tra «congegno partecipativo» e «congegno negoziale», proprio sulla base del collegamento tra le due «prassi», che si manifesta quando le forme di partecipazione nell'impresa e di coinvolgimento dei lavoratori sono «veicolate dalla contrattazione collettiva ${ }^{65}$.

63 M. BIAGI, Dalla nocività conflittuale alla sicurezza partecipata: relazioni industriali e ambiente di lavoro in Europa verso il 1992, in ID. (a cura di), Tutela dell'ambiente di lavoro e direttive Cee, cit., p. 133.

64 A. Peruldi, Intervento, in Rappresentanza collettiva dei lavoratori e diritti di partecipazione alla gestione delle imprese, cit., 305, secondo il quale «laddove si voglia, viceversa, individuare nella partecipazione un elemento olistico e omnicomprensivo, allora il sistema entra in sofferenza».

65 A. Alaimo, L'eterno ritorno della partecipazione: il coinvolgimento dei lavoratori al tempo delle nuove regole sindacali, cit., p. 313 ss. In merito ad «antagonismo e compatibilità tra partecipazione e contrattazione collettiva» cfr., in particolare, M. D’Antona, Partecipazione, codeterminazione, contrattazione, cit., p. 137 ss.; sul tema v. T. TREu, Cogestione e partecipazione, in DLRI, 1989, p. 597 ss. 
Come anticipato, il conflitto è in realtà radicato nelle relazioni industriali che connotano l'ordinamento interno; pertanto, il contratto collettivo mantiene la propria funzione regolativa degli interessi delle parti contrapposte ${ }^{66}$.

La prospettiva presa ora in esame presenta alcune peculiarità, dato che la fonte contrattuale offre una regolamentazione in melius che va ad arricchire le misure di prevenzione, in un'ottica di innalzamento dei livelli di sicurezza.

Poiché non si può ipotizzare il perseguimento di un interesse comune delle parti, che andrebbe ad intaccare l'autonomia, e finanche primazia, cui le stesse ambiscono, si può sostenere che tali soluzioni migliorative siano oggetto di un negozio, che si pone in alternativa all'iniziativa unilaterale del datore di lavoro e presuppone il comporsi del conflitto sulle più diverse materie, compresa la pericolosità del lavoro da scongiurare o ridurre.

Negli esigui casi indicati si giunge, pertanto, ad una sintesi e mediazione degli interessi (contrapposti) ed il conflitto viene a comporsi nel contratto ${ }^{67}$. Esattamente si configura un negozio o scambio tra maggiore produttività e maggior benessere, senza la necessità di una scelta netta tra contrapposizione e partecipazio$n e^{68}$.

66 M.G. Garofalo, Qualche osservazione su partecipazione e conflitto, in Rappresentanza collettiva dei lavoratori e diritti di partecipazione alla gestione delle imprese, cit., p. 358, secondo cui «l'interesse dell'imprenditore è strutturalmente in conflitto con quello dei lavoratori»e «per garantire la coesione sociale è necessario creare i meccanismi giuridici che consentano di comporre questo conflitto». In proposito v. R. Del Punta, Contrattazione e partecipazione, in A. Baylos Grau, B. Caruso, M. D'antona, S. Sciarra (a cura di), Dizionario di Diritto del Lavoro Comunitario, Bologna, Monduzzi, 1996, p. 481 ss., il quale sottolinea l'«antitesi strutturale di interessi» sottesa al metodo contrattuale. Sul tema cfr. G. GHEzzi, Una prospettiva di partecipazione conflittuale e non istituzionalizzata, in L'impresa al plurale. Quaderni della partecipazione, 1999, n. 3-4, p. 195 ss.

67 G. Giugni, S. Sciarra, voce Contrattazione collettiva, in Novissimo Digesto Italiano, Appendice, vol. II, Torino, Utet, 1981, p. 607; M.G. Garofalo, Qualche osservazione su partecipazione e conflitto, cit., p. 358. Cfr., altresì, le considerazioni di O. KAHN-Freund, Labour and the Law, London, Stevens \& Sons, 1977, p. 15, secondo cui «any approach to the relations between management and labour is fruitless unless the divergency of their interests is plainly recognised and articulated».

68 Cfr. G.G. Baland, Il contenuto dell'obbligo di sicurezza, in QDLRI, 1993, n. 14, p. 89 , secondo il quale la contrattazione collettiva può rappresentare «un 
In verità, le organizzazioni sindacali affrontano tradizionalmente altre e diverse questioni, come il salario; il che spiega, almeno in parte, le difficoltà legate all'avvio di tale meccanismo di scambio, verificandosi un accostamento di negoziati diversi (quello sulla retribuzione e quello relativo a condizioni migliorative dell'ambiente di lavoro) ${ }^{69}$.

I più ampi contenuti di protezione della salute dei lavoratori, oggetto della contrattazione collettiva aziendale, sono, in definitiva, il risultato di uno scambio attraverso un confronto con i soggetti collettivi.

Sicché, la «lente» attraverso cui leggere ed interpretare la più recente storia delle relazioni industriali continua ad essere quella del conflitto, proprio «perché al centro di qualsiasi progetto prevenzionale stanno l'organizzazione del lavoro e le più gelose prerogative dell'imprenditore» ${ }^{70}$.

I profili partecipativi inclusi nel contratto collettivo possono essere considerati, al pari di questo, «frutto di un compromesso con la controparte sociale» ${ }^{71}$, da cui scaturiscono modelli di relazioni tra le parti nuovi e più moderni. La contrattazione, in altri termini, diventa «elemento di partecipazione», soprattutto man

punto di convergenza di interessi che muovono da punti divergenti», ossia «in presenza delle opportune condizioni, uno strumento per la positiva evoluzione della tutela della sicurezza».

69 Cfr., in proposito, le riflessioni di V. Leccese, Il diritto sindacale al tempo della crisi. Intervento eteronomo e profili di legittimità costituzionale, in DLRI, 2012, p. 490. In ordine, invece, al rapporto tra ruolo del sindacato e responsabilità sociale d'impresa cfr. M. MAGnanI, Responsabilità sociale d'impresa e ruolo delle relazioni sindacali, in L. MontuschI, P. TulLINI (a cura di), Lavoro e responsabilità sociale dell'impresa, cit., p. 109 ss.

70 L. Montuschi, La sicurezza nei luoghi di lavoro ovvero l'arte del possibile, cit., p. 424, il quale, a commento del d.lgs. n. 626/1994, già osservava che non sarebbe stato facile «abbandonare una prassi che vede le parti collettive, anche aziendali, confrontarsi al tavolo negoziale piuttosto che cogestire il sistema delle misure di sicurezza».

71 R. Del Punta, Conclusioni II Sessione- "Consenso e dissenso nella rappresentanza e nel conflitto collettivo”, in M. Barbera, A. Perulli (a cura di), Consenso, dissenso e rappresentanza: le nuove relazioni sindacali, Padova, Cedam, 2014, p. 269. Con riferimento alla partecipazione dei lavoratori alla gestione aziendale cfr. M. Marazza, Saggio sull'organizzazione del lavoro, Padova, Cedam, 2002, p. 203, secondo cui lo strumento partecipativo è «funzionale ad un più equilibrato bilanciamento di interessi che nascono e rimangono contrapposti». 
mano che i suoi campi d'intervento si estendono fino a ricomprendere «temi sempre più essenziali nell'organizzazione del lavoro, come la sicurezza» ${ }^{72}$.

Il sindacato, come chiarito, in particolare in questi ultimi tempi, per mezzo della contrattazione di tipo integrativo, ha assunto un «ruolo attivo nell'interazione con l'impresa» al fine della «soluzione concordata» di aspetti che concernono l'organizzazione del lavoro ${ }^{73}$. Sebbene i rapporti tra datore di lavoro e lavoratori implichino usualmente posizioni di conflittualità, si deve rimarcare l'intento comune di favorire la realizzazione di una efficace politica di prevenzione e protezione dei rischi. Del resto, il perseguimento attraverso il veicolo contrattuale di un obiettivo comune, rectius di una «comunione di scopo» ${ }^{74}$, non contraddice, né elimina, «l'esistenza di interessi finali in conflitto» ${ }^{75}$.

L'arricchimento di misure e metodi di prevenzione è, in tal modo, costruito e sedimentato dentro le dinamiche del conflitto; anzi, è la contrapposizione che può essa stessa generare prassi di coinvolgimento, il cui effetto è di perseguire l'interesse dei lavoratori al benessere nel lavoro e dell'imprenditore ad aumentare il profitto. Forse una provocazione che non stravolgerebbe i fondamenti del sistema di relazioni industriali italiano, mantenendone le sue peculiarità.

Ne consegue che la fonte collettiva appare ampliata nei contenuti ed anche rafforzata ${ }^{76}$.

Quale, infine, il valore giuridico delle regole così introdotte?

Le misure migliorative ed aggiuntive inserite nei contratti collettivi aziendali, pur non dovute, assumono immediatamente valore giuridico vincolante.

72 G. Proia, La partecipazione dei lavoratori tra realtà e prospettive. Analisi della normativa interna, cit., p. 65 ss.

73 I. Regalia, Per una riconfigurazione dell'azione sindacale, in Quaderni di rassegna sindacale, 2015, n. 1, p. 189.

74 G. Giugni, Introduzione allo studio della autonomia collettiva, Milano, Giuffrè, 1960, p. 111.

75 M.G. Garofalo, Qualche osservazione su partecipazione e conflitto, cit., p. 359.

76 Si vedano le considerazioni sul punto di P. Tosi, Informazione, consultazione e partecipazione dei lavoratori nelle imprese e nei gruppi di imprese della $\mathrm{Cee}$, in Le ragioni del diritto. Scritti in onore di Luigi Mengoni, Milano, Giuffrè, 1995, p. 1319. 
Si deve, anzitutto, riflettere sulla qualificazione delle clausole negoziali in tema di tutela della salute, ossia sull'incerta collocazione delle misure migliorative a garanzia del benessere dei lavoratori tra le clausole normative oppure tra quelle obbligatorie del contratto collettivo.

V'è da precisare che questa distinzione non è affatto semplice, a fronte soprattutto dell'introduzione di clausole «nelle quali possono intrecciarsi elementi normativi ed elementi obbligatori ${ }^{77}$, cioè clausole c.d. miste o ambivalenti, volte a disciplinare le relazioni tra le parti sociali nell'ambito dell'azienda, nonché ad attribuire diritti in capo ai contraenti individuali. Come chiarito, «la linea distintiva tra i contenuti obbligatori e quelli normativi risulta in verità stemperata anche alla luce di un'evoluzione della contrattazione collettiva verso obiettivi di tutela più complessi ed articolati» $^{78}$.

In particolare con riferimento al contratto collettivo aziendale «l'interesse degli stipulanti» pare difficilmente «scindibile dalla disciplina del rapporto individuale» ${ }^{79}$.

Così, se da un lato va sottolineata la natura obbligatoria dell'istituzione di comitati congiunti o paritetici al fine della promozione della salute dei prestatori di lavoro, dall'altro non si può escludere la natura mista delle previsioni di tutele ulteriori ove sia precisato l'impegno ad una consultazione o esame congiunto con le organizzazioni sindacali. In tal senso può essere interpretata, ad esempio, la

77 P. LAMBERTUCCI, Tipologia, struttura e funzioni della contrattazione collettiva, in Trattato di diritto privato diretto da Mario Bessone, in F. CARINCI (a cura di), Il lavoro subordinato, tomo I, Il diritto sindacale, Torino, Giappichelli, 2007, p. 233. In merito alla parte obbligatoria del contratto collettivo v. G. GHEzzI, $L a$ responsabilità contrattuale delle associazioni sindacali. La parte obbligatoria del contratto collettivo, Milano, Giuffrè, 1963, p. 24 ss.

78 L. Corazza, Il nuovo conflitto collettivo. Clausole di tregua, conciliazione e arbitrato nel declino dello sciopero, Milano, Franco Angeli, 2013, p. 69 ss.; in proposito cfr. in particolare L. MARIUCCI, La contrattazione collettiva, Bologna, Il Mulino, 1985, p. 229 ss., il quale ha precisato che «non sembra utile ricorrere alla tradizionale suddivisione tra parte normativa e obbligatoria del contratto collettivo», non essendo «in grado di rappresentare adeguatamente la molteplicità di ruoli svolti dalla contrattazione e di tecniche negoziali da questa utilizzate».

79 A. LASSANDARI, Il contratto collettivo aziendale e decentrato, cit., p. 57, secondo cui «tra gli impegni che il datore assume nella dialettica intersindacale finisce con il comprendersi la stessa modalità di regolamentazione dei rapporti individuali». 
valorizzazione da parte della contrattazione collettiva dello sviluppo professionale dei lavoratori e la realizzazione di precipue iniziative di formazione in merito alla sicurezza ed all'ambiente di lavoro, attraverso incontri tra l'azienda e le rappresentanze sindacali diretti ad esaminare i contenuti del piano formativo ed a suggerire eventuali proposte di miglioramento ${ }^{80}$.

Ci si deve, altresì, interrogare sulla possibilità che un successivo contratto collettivo aziendale revochi per il futuro i trattamenti aggiuntivi precedentemente erogati, in quanto non «acquisiti», bensì passibili di modifiche anche sfavorevoli. Occorre, cioè, chiarire quali siano le regole contrattuali rientranti nell'obbligo datoriale a garanzia dei diritti, non semplici aspettative, dei prestatori di lavoro.

Una modifica in tal senso è considerata possibile sulla scorta di un ormai consolidato orientamento giurisprudenziale, che ha sancito l'intangibilità dei soli diritti quesiti entrati a far parte del patrimonio dei lavoratori, da cui vanno tenute distinte le mere aspettative o «pretese alla stabilità nel tempo di normative collettive più favorevoli» ${ }^{81}$.

Con riferimento al profilo della tutela prevenzionistica dei rischi lavorativi la riflessione potrebbe, però, essere condotta diversamente, escludendo finanche una modifica in peius. Infatti, quanto alla tutela della salute nei luoghi di lavoro le misure ulteriori entrano nell'obbligazione di sicurezza, configurandosi, ai sensi dell'art. 2087 c.c., come «necessarie a tutelare l'integrità fisica e la personalità morale dei prestatori di lavoro» in base al mutevole parametro del progresso tecnico. Le richiamate soluzioni si giustificano proprio in ragione di un'esigenza di miglioramento del sistema di sicurezza delineato nel d.lgs. n. 81/2008, come rimarcato negli stessi contratti collettivi citati in precedenza, e da questo punto di vista si collocano all'interno dell'art. 2087 c.c., integrandone il contenuto.

Si può, allora, affermare che tali misure finalizzate alla protezione di un diritto assoluto, che fa parte dei diritti sociali fonda-

80 Si veda, ad esempio, il contratto collettivo aziendale di GD S.p.a. del 1 dicembre 2011.

81 Cfr., ex multis, Cass. 29 ottobre 2015, n. 22126, in DeJure; Cass. 18 settembre 2007, n. 19351, in DeJure; Cass. 17 marzo 1999, n. 2429, in DeJure. Quanto in particolare al profilo del welfare aziendale sia consentito rinviare a M. VINCIERI, Attività sindacale e benessere organizzativo dei lavoratori, cit., p. 647. 
mentali, non sono, almeno astrattamente, revocabili; una loro cancellazione sarebbe, semmai, possibile solo nell'ipotesi di una contestuale sostituzione con altre soluzioni idonee ad assicurare effetti equivalenti. In sostanza ciò che attiene in senso stretto alla garanzia di un diritto primario, quale quello alla salute, non può essere pregiudicato dalla fonte successiva, essendo rivendicabile dal creditore di sicurezza.

Conclusivamente, si può anche riflettere in chiave problematica sul fatto che le misure contemplate nel contratto collettivo ed incidenti sull'organizzazione del lavoro e sulla gestione della sicurezza nell'ambiente lavorativo dovrebbero essere tenute in conto dal datore di lavoro nella stessa predisposizione del documento di valutazione dei rischi di cui all'art 28, comma 2, d.lgs. n. 81/2008. Le previsioni contrattuali dovrebbero, infatti, essere trasposte nel documento, in ispecie quali misure di prevenzione e protezione «opportune per garantire il miglioramento nel tempo dei livelli di sicurezza» (art. 28, comma 2, lett. c).

Si potrebbe sostenere che tali clausole contrattuali produrrebbero in tal modo effetti non solo sul piano civilistico, ma assumerebbero anche una valenza penalistica, «seppur indirettamente» ${ }^{82}$.

4. Contrattazione collettiva e prevenzione dei rischi nell'ordinamento spagnolo: un esempio affine

La tendenza a concretizzare ed implementare le misure di prevenzione all'interno di accordi collettivi, in ispecie aziendali, è riscontrabile anche in contesti affini al nostro, come quello spagnolo, nel quale in particolare la negociación colectiva de empresa, include spesso previsioni integrative a tutela della salute in senso lato dei prestatori di lavoro.

82 In questo senso si veda P. PASCUCCI, Le finalità e le tecniche di regolazione della flessibilità degli orari di lavoro, intervento presentato nell'ambito del Seminario «I giovani giuslavoristi e gli studi di diritto del lavoro. In memoria del Prof. Mario Giovanni Garofalo», organizzato dall'AIDLASS e dall'Università di Bari Aldo Moro (Bari, 11 novembre 2016). 
La stessa Ley de Prevención de Riesgos Laborales (31/1995), modificata da successivi interventi legislativi, prevede che le disposizioni normative in materia «avranno in ogni caso il carattere di diritto necessario minimo», potendo essere migliorate ed integrate da parte dei contratti collettivi (art. 2.2) ${ }^{83}$. Altre disposizioni della Ley contengono, inoltre, un esplicito rinvio alla contrattazione collettiva, quale fonte complementare, come nel caso dei sistemi di designazione dei Delegados de Prevención (art. 35.4 LPRL) ${ }^{84}$.

A venti anni dall'entrata in vigore della Ley de Prevención de Riesgos Laborales, è stato rimarcato come la tutela della sicurezza sia divenuto un aspetto fondamentale del rapporto di lavoro, ormai del tutto integrato nell'azione delle organizzazioni sindacali ${ }^{85}$. Il rinvio alla fonte contrattuale, per sua natura dinamica, offre, infatti, la possibilità di avvicinare ed adattare le misure di prevenzione dei rischi alla realtà dell'ambiente di lavoro della singola impresa o al settore produttivo di riferimento ${ }^{86}$.

83 Sul ruolo della contrattazione collettiva in materia di tutela della sicurezza sul lavoro nell'ordinamento spagnolo cfr., in particolare, N. Mendoza NAvas, El tratamiento convencional de la salud y la seguridad en el trabajo, Granada, Comares, 2013, p. 5 ss.; EAD., Prevención de riesgos laborales: la regulación convencional, Albacete, Bomarzo, 2007, p. 25 ss.; A. Garrigues Giménez, Crisis y primer embate de la reforma de la negociación colectiva: la seguridad y salud laboral y la posible deriva hacia el dumping preventivo, in I Working Papers di Olympus, n. 4/2012, p. 9 ss.; G. Tudela Cambronero, Y. Valdeolivas García, La seguridad y la salud laboral en la negociación colectiva, Informes y Estudios, Relaciones Laborales, n. 88, Ministerio de Trabajo e Inmigración, Madrid, 2009, p. 35 ss.

84 Cfr. J.A. Fernández Avilés, Reflexiones a los 20 años de vigencia de la Ley de Prevención de Riesgos Laborales: Mucho camino por recorrer, in Revista de Trabajo y Seguridad Social, 2016, p. 12 ss., secondo il quale «la negociación colectiva puede desplegar un papel importante de soporte para los delegados de prevención en el ejercicio de sus funciones legales».

85 J.R. Mercader Uguina, La ley de prevención de riesgos laborales, ventes años después, in Información Laboral, 2015, n. 8, p. 29 ss. In proposito cfr., inoltre, le considerazioni di J.C. García QuiÑones, Sicurezza e salute sul lavoro: una prospettiva generale nel contesto dell'ordinamento giuridico spagnolo, in A. PERULLI, V. BRINo (a cura di), Sicurezza sul lavoro. Il ruolo dell'impresa e la partecipazione attiva del lavoratore, Padova, Cedam, 2012, p. 163, secondo cui la materia è «suscettibile di essere negoziata collettivamente».

86 J.A. Fernández Avilés, Reflexiones a los 20 años de vigencia de la Ley de Prevención de Riesgos Laborales: Mucho camino por recorrer, cit., p. 12, il quale sottolinea come la negoziazione collettiva sia una «fuente privilegiada - por su adaptabilidad - de regulación de la prevención de riesgos laborales». Cfr., inoltre, 
V'è da rimarcare, tra i fini perseguiti nell'ambito della recente Estrategia Española de Seguridad y Salud en el Trabajo 2015-2020, il «potenziamento della negoziazione collettiva» attraverso accordi che promuovano «il coinvolgimento dei lavoratori e datori di lavoro nell'adempimento responsabile dei propri obblighi di prevenzione dei rischi».

Invero, va rilevata la propensione, non dissimile alla prassi in parte seguita nell'ordinamento italiano soprattutto in un recente passato, a riprodurre e ripetere nei testi contrattuali i precetti generali contenuti nella legge. A ciò, tuttavia, si affiancano contenuti migliorativi ed integrativi della negociación colectiva, che in tal modo contribuisce ad estendere la cultura della prevenzione; è stata, appunto, sottolineata l'attenzione prestata al tema della salud laboral e l'arricchimento dei contenuti negoziati ${ }^{87}$.

Si possono distinguere, così, clausole generiche, che riportano per lo più mere dichiarazioni d'intenti, prive di reale interesse, e clausole di carattere specifico, che al contrario individuano azioni e misure concrete al fine di ridurre i rischi di infortuni sul lavoro. Da quest'ultimo punto di vista, l'attenzione è rivolta a vari aspetti quali l'organizzazione delle visite mediche e la pianificazione dell'attività preventiva e della valutazione dei rischi. In funzione specificativa del quadro normativo si possono menzionare le previsioni contrattuali che determinano le modalità e la periodicità della valutazione

sul tema M. Álvarez de La Rosa, Ley y negociación colectiva en la regulación de la prevención de riesgos laborales, in Anales de la Facultad de Derecho, 2012, 29, p. 16 ss. Si veda il recente contratto collettivo di settore, XVIII Convenio colectivo general de la industria química del 3 agosto 2015, in BOE 19 agosto 2015, ove si precisa che «se pretende no sólo el cumplimiento de las obligaciones legales y las responsabilidades de los actores implicados en el marco de la empresa, sino también fomentar una nueva cultura de la prevención que avance en el desarrollo de la misma en el sector».

$87 \mathrm{Al}$ riguardo $\mathrm{F}$. Navarro NiETo, El tratamiento de la prevención de riesgos laborales en la negociación colectiva, in Temas Laborales, 2004, p. 13 ss. Cfr., altresì, J. García Blasco, A.L. DE Val Tena, La negociación colectiva en materia de prevención de riesgos laborales, in J.L. Monereo Pérez, C. Molina Navarrete (dir.), S. Olarte Encabo, J.A. Fernández Avilés (coord.), Tratado de prevención de riesgos laborales. Teoría y práctica, Madrid, Tecnos, 2015, p. 660 ss., ove si precisa che la contrattazione collettiva in materia assume una funzione «clarificadora, especializada, complementaria, de concreción y de desarrollo de la propia legislación especial». 
dei rischi, nonché quelle che adeguano la sorveglianza sanitaria «en función de los riesgos inherentes al trabajo», come previsto dall'art. 22 della $L e y^{88}$.

Come anticipato, non mancano però interventi della contrattazione collettiva aziendale in funzione realmente migliorativa rispetto alle previsioni legali. Si tratta, ad esempio, dell'introduzione di una «vigilancia de la salud complementaria», ossia a completamento della sorveglianza sanitaria obbligatoria, consistente in visite mediche ulteriori e specialistiche ${ }^{89}$.

Peculiare rilievo sta assumendo, inoltre, il profilo della protezione dei rischi psicosociali ${ }^{90}$. Diversi sono gli esempi di contratti collettivi di grandi aziende che si soffermano sul tema e contemplano un Protocolo de prevención y tratamiento de situaciones de acoso moral y sexual ${ }^{91}$.

V'è, altresì, da ricordare che nell'ordinamento spagnolo la contrattazione collettiva è espressamente regolata nel Título III dell' $E$ statuto de los trabajadores. I contratti collettivi c.d. statutari hanno efficacia normativa e sono vincolanti erga omnes, posto che «obligan a todos los empresarios y trabajadores incluidos dentro de su

88 L. Meléndez Morillo-Velarde, La prevención de riesgos laborales en la negociación colectiva, Navarra, Aranzadi, 2004, p. 35 ss. Sul tema della vigilancia de la salud cfr., in particolare, F.A. González DíAz, Límites empresariales en el desarrollo y ejecución de la obligación preventiva de vigilancia de la salud de los trabajadores, in C. Sánchez Trigueros, F.A. González Díaz (dir.), Libertad de empresa y poder de dirección del empresario en las relaciones laborales, Navarra, Aranzadi, 2011, p. 139 ss.

89 Cfr., in tal senso il Convenio colectivo de Retevisión I, SAU, del 6 maggio 2014, in BOE 19 maggio 2014, ove è specificato che «la dirección promoverá campañas de medicina preventiva».

${ }_{90}$ Quanto al profilo della tutela negoziale dei rischi psicosociali v. J.A. FERNÁNDez Avilés (dir.), La negociación colectiva en Prevención de Riesgos Psicosociales en el Trabajo, UGT-CEC, 2015; con riguardo, invece, ad una analisi giurisprudenziale dei rischi psicosociali v. M.L. ARASTEY SAHÚn, Los riesgos psicosociales en la apreciación de los tribunales: algunas cuestiones sobre la aplicación práctica de la Directiva marco 1989/391/CEE, in Relaciones Laborales, 2012, p. 85 ss.

91 Cfr. il Convenio colectivo de Distribuidora de Televisión Digital, SA y Compañia Independiente de Televisión, SL (antes Grupo Sogecable), del 13 maggio 2014, in BOE 27 maggio 2014; negli stessi termini v., ad esempio, il Convenio colectivo de Flex Equipos de Descanso, SAU, del 18 aprile 2013, in BOE 10 maggio 2013 e il Convenio colectivo de Supercor, SA y Tiendas de Conveniencia, SA, del 2 aprile 2013, in BOE 18 aprile 2013. 
ámbito de aplicación y durante todo el tiempo de su vigencia» (art. 82.3 ET). Sicché, nella gerarchia delle fonti, hanno la natura giuridica di norme subordinate alla Costituzione ed alle disposizioni legali e regolamentari dello Stato $^{92}$.

Si deve, allora, concludere che nelle ipotesi di clausole negoziali (statutarie) migliorative ci si può riferire a buone pratiche accordate con il consenso di entrambe le parti o, più correttamente, a buoni contenuti normativi in materia di prevenzione dei rischi.

\section{La «partecipazione equilibrata» nel contesto italiano}

Un rilevante impulso nella direzione del coinvolgimento/partecipazione dei lavoratori si coglie, senza dubbio, nella direttiva quadro 89/391/CEE, ove è rimarcata l'indispensabilità di una «partecipazione equilibrata» degli stessi lavoratori e/o dei loro rappresentanti, conformemente alle legislazioni e prassi nazionali. Da tempo è attuale, dunque, l'invito alla previsione di procedure e strumenti adeguati che, secondo le parole della direttiva, possano sviluppare «l'informazione, il dialogo e la partecipazione equilibrata» tra le parti, al fine di garantire un miglior livello di protezione nel lavoro.

Pare evidente che l'incertezza che connota il termine «partecipazione equilibrata», sul quale la dottrina si è diffusamente soffermata, sia dovuta ad una usuale «scelta - non scelta del legislatore eu-

92 Occorre, tuttavia, precisare che dai contratti collettivi statutari vanno tenuti distinti i contratti extrastatutari, stipulati nel rispetto dell'art. 37, comma 1, della Costituzione spagnola, ma al di fuori della regolamentazione dell'Estatuto de los trabajadores, con efficacia limitata ai lavoratori e datori di lavoro rappresentati dalle parti contraenti. In merito al sistema della contrattazione collettiva ed alle recenti riforme sul tema cfr. J. Cruz ViLlalón, Impacto de las reformas laborales sobre la negociación colectiva, in Temas laborales, 2013, n. 120, p. 13 ss.; C.L. ALFONSO MELLADo, La reforma de la negociación colectiva en la Ley 3/2012: especial referencia a la negociación en la empresa, la estructura de la negociación y la inaplicación de los convenios, in Relaciones Laborales, 2013, 3, p. 15 ss. In generale sul sistema di relazioni industriali nell'ordinamento spagnolo v. F. VALDÉs DAL-RÉ, Le relazioni industriali nel sistema spagnolo: una panoramica generale, in L. FIoRillo, A. Perulli (dir.), Le relazioni industriali, Torino, Giappichelli, 2014, vol. III, p. 299 ss. 
ropeo», cioè alla finalità di abbracciare varie soluzioni, nel rispetto delle specificità dei diversi Paesi membri ${ }^{93}$. Pertanto questo generico riferimento al tema della partecipazione deve essere calato all'interno dei sistemi nazionali e valutato secondo gli specifici modelli di rappresentanza dei lavoratori ivi presenti.

Sono stati messi in risalto alcuni tratti distintivi che indicativamente assume «l'elemento chiave» della partecipazione in materia di salute e sicurezza sul lavoro, tra i quali la specializzazione delle rappresentanze, nonché la loro precipua funzione di promuovere l'adempimento e di migliorare gli standard di sicurezza nei luoghi di lavoro ${ }^{94}$.

La prospettiva fatta propria dalla direttiva quadro e contenuta nel suo articolo 11 è quella del coinvolgimento, nella gestione delle misure di protezione, sia dei singoli lavoratori sia delle loro rappresentanze con funzioni specifiche. La scelta del legislatore comunitario è stata di andare oltre a una vera ed effettiva consultazione, che sia cioè preventiva e tempestiva, tale da permettere al datore di lavoro di tenere in conto eventuali osservazioni e proposte; la partecipazione c.d. «bilanciata» coinciderebbe con una forma di codecisione tra le parti in merito a qualunque azione che possa avere effetti rilevanti sulla sicurezza e sulla salute ${ }^{95}$. Appare, quindi, non equivoca-

93 L. Zoppoli, Rappresentanza collettiva dei lavoratori e diritti di partecipazione alla gestione delle imprese, cit., p. 430; cfr., inoltre, P. TuluINI, Sicurezza sul lavoro e modello sociale europeo: un'ipotesi di sviluppo, cit., p. 1270, secondo la quale il principio della «partecipazione equilibrata» è un «principio di armonizzazione» «ancora tanto controverso quanto carico di possibili implicazioni per il sistema italiano di relazioni industriali». Cfr. sul tema M. BIAGI, Dalla nocività conflittuale alla sicurezza partecipata: relazioni industriali e ambiente di lavoro in Europa verso il 1992, in ID. (a cura di), Tutela dell'ambiente di lavoro e direttive Cee, cit., p. 132 ss.

94 E. AlEs, Occupational Health and Safety: a European and Comparative Legal Perspective, in WP C.S.D.L.E. "Massimo D'Antona”. INT, 120/2015, p. 36 ss.

95 In tal senso v. M. BIAGI, Dalla nocività conflittuale alla sicurezza partecipata: relazioni industriali e ambiente di lavoro in Europa verso il 1992, cit., p. 133; ID., Rappresentanza e democrazia in azienda. Profili di diritto sindacale comparato, cit., p. 222; cfr., altresì, E. Ales, L'articolo 9 Statuto dei lavoratori alla luce della legislazione più recente in materia di salute e sicurezza: partecipazione o controllo?, in Studi in onore di Tiziano Treu. Lavoro, istituzioni, cambiamento sociale, II, Contratti di lavoro, Napoli, Jovene, 2011, p. 1053, il quale ha precisato che la partecipazione «nella sua versione equilibrata, secondo quanto espressamente dispo- 
bile la direzione intrapresa dalla direttiva quadro verso «un modello di partecipazione codeterminativo ${ }^{96}$.

Le forme che nell'ordinamento italiano ha assunto la partecipazione dei lavoratori sono, come noto, alquanto deboli e si manifestano, essenzialmente, nella loro diretta formazione e informazione, nonché nella consultazione seppur attraverso i loro rappresentanti. I lavoratori «uti singuli» hanno, fin dal d.lgs. n. 626/1994, un ruolo defilato, contribuendo «più modestamente» all'adempimento degli obblighi previsti a tutela della salute e sicurezza sui luoghi di lavoro (cfr. l'art. 20, d.lgs. n. 81/2008) ${ }^{97}$.

Il ruolo di "protagonisti" è insomma attribuito ai rappresentanti dei lavoratori per la sicurezza, titolari di poteri e diritti ulteriori rispetto ai compiti in precedenza già propri delle rappresentanze costituite ai sensi dell'art. 9 dello Statuto dei lavoratori, di cui deve ammettersi l'abrogazione, quantomeno implicita, nonostante la questione abbia suscitato un vivace dibattito in dottrina fino a tempi recenti ${ }^{98}$.

L'art. 9 St. lav. ha rappresentato «il primo riconoscimento legislativo dell'interesse collettivo alla sicurezza del lavoro», attraver-

sto dall'art. 11 dir. 89/391/CE, si distingue dalla consultazione» che rappresenta un «istituto a se stante».

96 B. Caruso, Introduzione, in B. Caruso, M. D’Antona, S. Sciarra (a cura di), Il diritto del lavoro nell'Unione europea. Francia, Germania, Gran Bretagna, Spagna, cit., p. 167.

97 Così già a commento del d.lgs. n. 626/1994 cfr. L. MonTuSCHI, voce Ambiente di lavoro, in Digesto delle Discipline Privatistiche, Sezione Commerciale, Torino, Utet, 2000, p. 10.

98 A favore del superamento dell'art. 9 St. lav. cfr. G. Proia, Consultazione e partecipazione dei lavoratori, in L. Montuschi (a cura di), Ambiente, Salute e Sicurezza. Per una gestione integrata dei rischi da lavoro, cit., p. 200, secondo cui «non residua spazio per la sopravvivenza della stringata regolamentazione di cui all'art. 9, St. lav.», che risulta assorbita dalla legge più recente e dettagliata. Negli stessi termini vedi P. PAscucci, Salute e sicurezza: dalle rappresentanze dell'art. 9 ai rappresentanti del d.lgs. $n$. 81/08, in DLM, 2010, n. 3, p. 664; C. Zoli, Sicurezza del lavoro: contrattazione e partecipazione, in RGL, 2000, p. 622; A. TAMPIERI, Sicurezza sul lavoro e modelli di rappresentanza, Torino, Giappichelli, 1999, p. 122; G. NatulLo, Rappresentanze dei lavoratori per la sicurezza e rappresentanze sindacali in azienda, in ADL, 1997, p. 209; L. GaLANTINo, Il contenuto dell'obbligo di sicurezza, in EAD. (a cura di), La sicurezza del lavoro. Commento al decreto legislativo 19 settembre 1994, n. 626, Milano, Giuffrè, 1996, p. 34. Contra v. M.G. Garofalo, La legislazione nel 1993-1994, in DLRI, 1995, p. 110. 
so la previsione di precipue rappresentanze dei lavoratori, che hanno anticipato la figura del rappresentante dei lavoratori per la sicurezza, di cui al d.lgs. n. 81/2008 ${ }^{99}$. Quanto all'attuale vigenza della norma, non si è mancato di chiarire che il modello di rappresentanza prefigurato dall'art. 9 St. lav., «di matrice conflittuale», è distante da quello comunitario «ispirato al principio della prevenzione partecipata ${ }^{100}$ ed ormai superato dalle successive disposizioni incluse già nel d.lgs. n. 626/1994 ed a fortiori nel d.lgs. n. 81/2008. La norma statutaria, dunque, pur avendo «inaugurato la fase della gestione collettiva della sicurezza nei luoghi di lavoro», può considerarsi assorbita nella disciplina prevenzionistica di derivazione comunitaria ${ }^{101}$.

\subsection{Il rappresentante dei lavoratori per la sicurezza}

La partecipazione debole recepita nell'ordinamento interno attribuisce ai lavoratori e/o loro rappresentanti il mero diritto all'informazione e consultazione, rimanendo sul «terreno della procedimentalizzazione» ${ }^{102}$. Si tratta tendenzialmente di meccani-

99 M. LaI, I soggetti collettivi (Rappresentanze per la sicurezza; Organismi paritetici): costituzione, prerogative, attività, in G. NATULlo (a cura di), Salute e sicurezza sul lavoro, cit., p. 799 ss. Sulla rilevanza dell'art. 9 St. lav. cfr., in particolare, G. GHEZzI, Una prospettiva di partecipazione conflittuale e non istituzionalizzata, cit., p. 204 ss.; v., inoltre, le considerazioni di G.G. BALANDI, Individuale e collettivo nella tutela della salute nei luoghi di lavoro: l'art. 9 dello statuto, cit., p. 225 ss.

100 P. Pascucci, Salute e sicurezza: dalle rappresentanze dell'art. 9 ai rappresentanti del d.lgs. $n$. 81/08, cit., p. 664. Sul tema cfr. L. GAETA, Che cosa è oggi lo Statuto dei lavoratori?, in $L D, 2010$, n. 1, p. 50 ss., il quale, con riguardo alle norme in materia di tutela della salute nei luoghi di lavoro contenute nello Statuto dei lavoratori, precisa trattarsi di «articoli che possono dirsi completamente, o pressoché, sostituiti, anche se quasi mai abrogati testualmente, dall'evoluzione normativa registratasi in materia». In ordine all'«attualità» della normativa statutaria cfr. le riflessioni di F. MARTELloni, Inventario di sguardi sullo Statuto dei lavoratori, in $L D, 2010$, n. 4, p. 361 ss.

101 L. MontuschI, L'incerto cammino della sicurezza del lavoro fra esigenze di tutela, onerosità e disordine normativo, cit., p. 510.

102 C. Zoli, Le clausole di procedimentalizzazione dei poteri imprenditoriali, in M. D’Antona (a cura di), Letture di diritto sindacale, Napoli, Jovene, 1990, p. 389 ss. 
smi partecipativi che non vanno oltre una attenta informazione dei prestatori di lavoro e un coinvolgimento dei loro rappresentanti, $i$ quali non possono, però, prendere parte alle decisioni determinanti l'organizzazione del lavoro. Un'esperienza in prevalenza di tipo procedurale, che non sacrifica, tuttavia, la «centralità della dimensione collettiva», rafforzata dal d.lgs. n. 81/2008 ${ }^{103}$.

Questa forma di "partecipazione" non si esplica nell'ambito di strutture di confronto a composizione mista, alquanto diffuse invece a livello europeo. Tale rappresentanza è istituita in tutte le aziende o unità produttive e differenziata quanto alla sua composizione in ragione del numero degli occupati: solo ove siano presenti più di quindici lavoratori il rappresentante per la sicurezza è «eletto o designato» dai prestatori di lavoro «nell'ambito delle rappresentanze sindacali in azienda», diversamente, invece, è «di norma eletto direttamente dai lavoratori al loro interno» (art. 47, d.lgs. n. 81/2008). In proposito, nonostante la scelta accolta negli accordi interconfederali in materia per una «sindacalizzazione pressoché completa dell'organismo di rappresentanza aziendale per la sicurezza», è stata negata una assoluta corrispondenza tra rappresentanti dei lavoratori per la sicurezza (RLS) e rappresentanze sindacali ${ }^{104}$, come del resto già a suo tempo con riferimento all'art. 9 St. lav. ${ }^{105}$.

È opportuno, a questo punto, valutare le attribuzioni e prerogative riservate dal legislatore a questa figura, mettendo in luce le possibilità ad esso riconosciute di "intromissione" e "cooperazione" nella gestione dell'organizzazione della prevenzione, quindi delle misure necessarie a garantire il benessere all'interno dell'ambiente di lavoro.

Deve essere rimarcato che alle rappresentanze collettive per la sicurezza sono devoluti compiti non negoziali, ma prevalentemente consultivi, propositivi, in merito all'attività di prevenzione, e di con-

103 C. Zol, Artt. 47-50, in ID. (a cura di), Principi comuni, in L. MontuschI (dir.), La nuova sicurezza sul lavoro, cit., p. 509 ss.

104 C. Zoli, Artt. 47-50, in ID. (a cura di), Principi comuni, cit., p. 512, secondo il quale il RLS è «un organo specialistico dotato di funzioni peculiari, che richiedono autonomia e competenza» ed «è espressione dell'intera comunità dei lavoratori di un'azienda o di un'unità produttiva».

105 In tal senso G. Ghezzi, Art. 9, in G. Ghezzi, G.F. Mancini, L. Montuschi, U. Romagnol, Statuto dei diritti dei lavoratori, Bologna-Roma, Zanichelli-Il Foro italiano, 1972, p. 145 ss. 
trollo. Assumono, altresì, rilevanza i diritti di formazione e informazione di cui i RLS sono destinatari; l'informazione ricevuta inerisce alla valutazione dei rischi ed alle misure di sicurezza relative e precede la fase consultiva.

La consultazione «preventiva e tempestiva» è prevista in ordine alla valutazione dei rischi, all'individuazione, programmazione, realizzazione e verifica della prevenzione all'interno dell'azienda o unità produttiva, alla designazione del medico competente, del responsabile e degli addetti al servizio di prevenzione, all'attività di prevenzione incendi, al primo soccorso, alla evacuazione dei luoghi di lavoro, nonché in ordine all'organizzazione della formazione (art. 50, comma 1, lett. b, c, d, d.lgs. n. 81/2008) ${ }^{106}$. Il momento consultivo tuttavia, come sottolineato, si estrinseca in «uno scambio di pareri e si arresta sulla soglia di una vera e propria trattativa, che nella specie il datore di lavoro non ha l'obbligo di attivare» ${ }^{107}$.

Non poca importanza è da attribuire alla partecipazione del RLS, insieme al datore di lavoro e agli altri soggetti della sicurezza, alla riunione periodica ai sensi dell'art. 35, d.lgs. n. 81/2008, quale momento essenziale di partecipazione collettiva nella gestione della prevenzione dei rischi, anzi il «solo diritto di partecipazione» riconosciuto al RLS ${ }^{108}$; riunione che costituisce il luogo di incontro ove è preso in esame e rivalutato il sistema di organizzazione della prevenzione e nel corso della quale possono essere individuati codici di comportamento e buone prassi, al fine del continuo miglioramento delle condizioni di salute sul lavoro.

Ne deriva che insieme all'istituzione rappresentativa dei prestatori di lavoro v'è la possibilità di organizzare il presente e di progettare il futuro all'interno dell'azienda.

È stato messo in luce il rafforzamento da parte del decreto del

106 L'inadempimento dell'obbligo di consultazione del RLS, richiamato nell'art. 18 , comma 1 , lett. s, d.lgs. n. $81 / 2008$, è sanzionato dall'art. 55, comma 5, lett. e, del medesimo decreto con la previsione di un'ammenda da 2000 a 4000 euro.

107 C. Zoli, Artt. 47-50, in ID. (a cura di), Principi comuni, cit., p. 519; cfr., inoltre, ID., Gli obblighi a trattare nel sistema dei rapporti collettivi, Padova, Cedam, 1992, p. 122 ss.

108 E. Ales, L'articolo 9 Statuto dei lavoratori alla luce della legislazione più recente in materia di salute e sicurezza: partecipazione o controllo?, cit., p. 1054. 
2008 del ruolo attribuito al rappresentante dei lavoratori; sono, in tal senso, oltreché moltiplicate le strutture rappresentative per la sicurezza, anche ulteriormente valorizzate le funzioni ad esse assegnate, con una accentuazione dell'azione sindacale a tutela della sicurezza del lavoro ${ }^{109}$.

Può, allora, il rappresentante dei lavoratori contribuire realmente a migliorare la sicurezza nei luoghi di lavoro?

L'accento potrebbe essere enfaticamente posto sulla previsione, non nuova bensì già presente nel d.lgs. n. 626/1994, che assegna al RLS la facoltà di promuovere «l'elaborazione, l'individuazione e l'attuazione delle misure di prevenzione idonee a tutelare la salute e l'integrità fisica dei lavoratori» (art. 50, comma 1, lett. h, d.lgs. n. 81/2008). Pur trattandosi, appunto, di una mera funzione propositiva, si può intravedere l'intenzione di fornire alle rappresentanze collettive dei lavoratori il potere di elaborare misure anche aggiuntive, destinate a garantire la protezione nell'ambiente di lavoro. Del resto, ai sensi dell'art. 51, comma 3, è esplicitamente attribuito agli organismi paritetici un (potenziale) ruolo «promozionale e di supporto tecnico alle imprese» ${ }^{110}$ nell'elaborazione di misure e soluzioni migliorative della sicurezza sul lavoro.

Si può scorgere in entrambe le previsioni un'assonanza con il disposto dell'art. 2087 c.c., proteso alla continua ricerca delle misure «necessarie», o appunto «idonee», alla protezione della salute. Rappresentanti per la sicurezza ed organismi paritetici, rispettivamente a livello aziendale e territoriale, possono essere figure decisive, soprattutto alla luce delle integrazioni effettuate dal decreto $\mathrm{n}$. $81 / 2008$.

Non sono, in verità, mancati rilievi critici da parte della dottrina, che ha chiarito come le disposizioni del decreto del 2008 riferentisi alla «consultazione e partecipazione» non introducono reali diritti di partecipazione in capo al rappresentante dei lavoratori

109 In tal senso v. G. Natullo, Il quadro normativo dal Codice civile al Codice della sicurezza sul lavoro. Dalla Massima Sicurezza possibile alla Massima Sicurezza effettivamente applicata?, cit., p. 34.

$110 \mathrm{M}$. LAI, Il sistema della rappresentanza e delle relazioni collettive, in P. PAscucci (a cura di), Il d.lgs. n. 81/2008: due anni dopo. I "sistemi" del diritto della sicurezza sul lavoro, cit., p. 54. 
per la sicurezza, sembrando difficile «argomentare in favore di un coinvolgimento effettivo del RLS nella gestione della sicurezza» ${ }^{111}$. All'implementazione ed all'arricchimento della disciplina ad opera del d.lgs. n. 81 è stata attribuita una rilevanza «proposta più sul piano normativo astratto che su quello concreto, in termini cioè di effetti sull'incremento dei reali livelli di tutela nelle aziende» ${ }^{112}$.

Un incentivo verso un maggior confronto con i rappresentanti dei lavoratori per la sicurezza si coglie, invece, come già accennato, nei contenuti della contrattazione collettiva aziendale, così da consentire la migliore gestione comune delle problematiche relative alla sicurezza degli ambienti di lavoro. Affiora, in tal modo, la «volontà di implementare in maniera congiunta (azienda/RLS) l'analisi dei programmi di valutazione e prevenzione», tenendo in primo luogo presente proprio le segnalazioni formulate dai rappresentanti dei lavoratori, anche al fine di «individuare soluzioni transitorie atte a garantire la massima sicurezza possibile» ${ }^{113}$. Il coinvolgimento e la valorizzazione della figura del rappresentante dei lavoratori per la sicurezza è tra i propositi perseguiti da parte della fonte negoziale aziendale.

In definitiva, pur non dubitandosi che tale quadro regolativo sia «ispirato al principio della "prevenzione partecipata" ${ }^{114}$, è anche vero che molta strada è ancora da fare nella direzione di un coinvolgimento effettivo al processo decisionale da parte dei titolari del credito di sicurezza.

111 E. Ales, L'articolo 9 Statuto dei lavoratori alla luce della legislazione più recente in materia di salute e sicurezza: partecipazione o controllo?, cit., p. 1054.

112 G. Natullo, Il quadro normativo dal Codice civile al Codice della sicurezza sul lavoro. Dalla Massima Sicurezza possibile alla Massima Sicurezza effettivamente applicata?, cit., p. 33.

${ }_{113} \mathrm{Si}$ veda in tal senso il contratto collettivo aziendale di GD S.p.a. del 1 dicembre 2011; nonché il contratto collettivo aziendale di EasyJet Airline Company Limited del 4 marzo 2015, ove le parti, «in uno spirito di reciproca e fattiva collaborazione finalizzata a promuovere e migliorare la sicurezza e la salute dei lavoratori» ed a «costruire una comune visione di valutazione e gestione dei rischi», confermano «la primaria importanza rivestita dalla formazione dei RLS»; il contratto collettivo aziendale di Délifrance Italia del 19 gennaio 2016; il contratto integrativo aziendale di Ikea del 21 gennaio 2016.

114 P. PAScucci, Salute e sicurezza: dalle rappresentanze dell'art. 9 ai rappresentanti del d.lgs. $n$. 81/08, cit., p. 664; ID., Prevenzione, organizzazione e formazione. A proposito di un recente libro sulla sicurezza sul lavoro, in DSL, 2016, n. 1, p. 76 . 
Ciò che manca nella normativa interna, accomunata in tal senso ad altri ordinamenti, è proprio il meccanismo di codecisione ossia di partecipazione (forte) ad una decisione assunta in accordo tra le parti, che realizzi l'influenza dei lavoratori, o loro rappresentanze, sui poteri e sulla gestione, altrimenti esclusiva, del management. Tale forma di partecipazione richiama evidentemente il concetto di democrazia industriale, su cui ampio e animato è stato il dibattito in un recente passato.

Si è ancora lontani, in sostanza, dal dover pervenire, per imposizione di legge, ad un accordo comune a lavoratori e controparte datoriale o quantomeno dal poter realmente incidere sull'assunzione delle decisioni, che rimangono, alla fine, solo in capo al datore di lavoro, unico responsabile di garantire la sicurezza ed il benessere nell'ambiente lavorativo ${ }^{115}$.

115 L. Montuschi, L'incerto cammino della sicurezza del lavoro fra esigenze di tutela, onerosità e disordine normativo, cit., p. 515. 
CAPITOLO III

\section{IL RILIEVO DELLA DIMENSIONE PARTECIPATIVA IN ALTRI ORDINAMENTI NAZIONALI}

SOMMARIO: 1. La diversa interpretazione del meccanismo partecipativo ai fini della tutela della sicurezza nei luoghi di lavoro. - 2. Il dovere di consultazione dei rappresentanti dei lavoratori nel Regno Unito e l'ipotesi del Safety Committee. - 3. Il Comité d'hygiène, de sécurité et des conditions de travail. - 4. Il Comité de Seguridad y Salud. - 5. Gli accordi di codeterminazione nella tutela della sicurezza sul lavoro (Betriebsvereinbarungen). - 5.1. Gli accordi aziendali volontari (freiwillige Betriebsvereinbarungen). - 6. Brevi riflessioni conclusive.

1. La diversa interpretazione del meccanismo partecipativo ai fini della tutela della sicurezza nei luoghi di lavoro

Occorre soffermarsi sui meccanismi partecipativi disciplinati nei diversi contesti nazionali.

Il tema della partecipazione e del coinvolgimento dei lavoratori nella tutela della loro salute, come anticipato, si presta particolarmente ad uno studio comparato. V'è, tuttavia, la consapevolezza che non è facile «individuare modelli normativi o istituti comuni, o anche solo dominanti, nel campo dei rapporti collettivi di lavoro», in ragione delle peculiarità dei vari sistemi di relazioni industriali ${ }^{1}$.

1 A. Vimercati, B. Veneziani, La partecipazione in Europa tra Costituzione, legge e contrattazione collettiva, in L'impresa al plurale. Quaderni della partecipazione, 1999, n. 3-4, p. 279 ss.; cfr., inoltre, le riflessioni di O. KAHN-Freund, Sull'uso ed abuso del diritto comparato, in RTDPC, 1975, p. 785. 
Da tempo sono state sottolineate le difficoltà della comparazione e forti sono tuttora i dubbi in merito alla possibilità di fare «vera comparazione», considerate le «diversità enormi tra regole del diritto sostanziale e processuale in giro per il mondo», nonché $\mathrm{i}$ «fattori extra-giuridici, sociali, economici, culturali» definiti «imprevedibili» ${ }^{2}$.

In effetti è stato constatato il «limite culturale», consistente nella «diffusa propensione a fondare un modello universale di relazioni industriali, applicabile in modo indiscriminato a tutti i paesi che si ritiene di poter definire come paesi industriali avanzati» ${ }^{3}$. Ciò è tanto più vero se ci si riferisce al sistema tedesco della Mitbestimmung, al centro di un rinnovato interesse da parte degli studiosi in questi ultimi anni, quale esempio da perseguire nell'ottica di forme partecipative di relazioni industriali ${ }^{4}$.

Il meccanismo partecipativo promosso a livello europeo è stato diversamente interpretato nei singoli ordinamenti. Si deve anticipare che il coinvolgimento dei lavoratori è realizzato oltreché attraverso rappresentanti specializzati presenti a livello aziendale, come nel caso dell'ordinamento italiano, anche attraverso la costituzione di appositi comitati nei quali alla rappresentanza dei lavoratori si affianca quella datoriale: così è previsto, invece, a titolo esemplificativo nel contesto francese, spagnolo e belga.

Non si tratta, invero, di novità, né del mero adattamento delle legislazioni interne ai principi comunitari. Occorre, infatti, ricordare che in molti ordinamenti le previsioni legislative che si riferiscono

2 R. Salomone, Metodo comparato e misurazione del diritto del lavoro, in F. BASEnghi, L.E. Golzio (a cura di), Regole, politiche e metodo. L'eredità di Marco Biagi nelle relazioni di lavoro di oggi, Torino, Giappichelli, 2013, p. 77 ss.; cfr. M. BIAGI, Cultura e istituti partecipativi delle relazioni industriali in Europa, cit., p. 291, il quale ha sottolineato le evidenti difficoltà nel mettere a confronto sistemi contraddistinti da «fattori politico-economici, sociali e istituzionali specifici».

3 F. Galgano, L'impresa, il sistema economico, la partecipazione operaia, in C. Smuraglia, C. Assanti, F. Galgano, G. Ghezzi, La democrazia industriale. Il caso italiano, cit., p. 31.

4 Secondo M. BIASI, La partecipazione dei lavoratori alla gestione e la Mitbestimmung tedesca, in C. Zoli (a cura di), Lavoro e impresa: la partecipazione dei lavoratori e le sue forme nel diritto italiano e comparato, cit., p. 33, per avvicinarsi a tale modello regolativo occorre comprendere $\mathrm{i}$ «fattori storico/culturali», nonché «politici», che hanno portato «alla sua evoluzione da un modello conflittuale verso le attuali forme solidaristiche e collaborative». 
a specifiche rappresentanze dei lavoratori ed anche a commissioni o comitati sulle questioni di salute sul lavoro, sono risalenti nel tempo e precedono il recepimento della stessa direttiva quadro 89/391.

Si può menzionare la legislazione francese, che già nel 1982 con la Loi n. 82-1097 ha regolato l'istituzione del Comité d'hygiène, de sécurité et des conditions de travail, decretandone la sua indispensabilità nelle problematiche attinenti alla sicurezza.

Anche l'ordinamento spagnolo, prima dell'attuale Ley de Prevención de Riesgos Laborales del 1995, che detta le disposizioni di riferimento in relazione al Comité de Seguridad y Salud, aveva regolato precedentemente la figura del Comité de Seguridad e Higiene en el Trabajo ad opera del decreto n. 432/19715.

Quanto alla Repubblica Federale Tedesca, l'Arbeitssicherheitsgesetz (Asig), ossia la legge del 1973, successivamente modificata, sui medici aziendali, gli ingegneri per la sicurezza ed altri specialisti in materia di sicurezza sul lavoro, ha previsto, all'art. 11, la necessità di individuare un c.d. Comitato per la sicurezza del lavoro ( $A r-$ beitsschutzausschuß), organismo specifico a livello d'azienda ove vi siano almeno venti lavoratori, formato dal datore di lavoro o un suo rappresentante, da due componenti designati dal consiglio d'azienda, dai medici aziendali, dagli addetti alla sicurezza e dai Sicherheitsbeauftragten, cioè i soggetti incaricati della sicurezza ai sensi del § 22 Sozialgesetzbuch VII (il Settimo Libro del Codice Sociale) ${ }^{6}$.

Con riguardo al Regno Unito si deve precisare che il Robens Committee, nominato nel 1970 dal Secretary of State for Em-

5 In proposito cfr. M.N. Moreno VIDA, Los derechos de participación y representación de los trabajadores en materia de prevención de riesgos. El Delegado de prevención, in J.L. Monereo Pérez, C. Molina Navarrete (dir.), S. Olarte Encabo, J.A. Fernández Avilés (coord.), Tratado de prevención de riesgos laborales. Teoría y práctica, cit., p. 636, che ha definito il Comité de Seguridad e Higiene en el Trabajo come un «tercer canal representativo».

6 L'Arbeitsschutzausschuß ha il compito di fornire consigli sulle questioni inerenti alla prevenzione dei rischi sul lavoro e si riunisce almeno una volta a quadrimestre. In particolare, tra le materie oggetto di consultazione vi sono la realizzazione di programmi per la tutela della salute e la pianificazione delle misure di sicurezza, l'esame delle denunce degli infortuni, la partecipazione alle regolari ispezioni aziendali. Cfr. per un commento R. AufHauser, § 11 Arbeitsschutzausschuß, in R. Aufhauser, H. BrunhöBer, P. Igl, Arbeitssicherheitsgesetz. Handkommentar, Baden-Baden, Nomos, 2010, p. 114 ss. 
ployment per rivedere le disposizioni sulla protezione della salute dei lavoratori, aveva già statuito che la partecipazione dei lavoratori era essenziale per l'effettivo funzionamento della gestione della salute e della sicurezza nel lavoro ${ }^{7}$. L'Health and Safety at Work Act, risalente al 1974, ha poi influenzato l'introduzione di normative similari in altri Paesi e perfino, come precisato in dottrina, l'emanazione della stessa direttiva quadro del $1989^{8}$.

È opportuno, dunque, delineare le principali caratteristiche che contraddistinguono, da questo punto di vista, i contesti europei ai quali si è scelto di fare riferimento.

Nel recepimento del monito comunitario, la partecipazione dei prestatori di lavoro ha assunto svariate forme, più o meno accentuate, includendo la mera informazione e consultazione, fino alla codeterminazione. I creditori della sicurezza sono coinvolti direttamente o attraverso le loro rappresentanze, sindacali e non, oppure agiscono per mezzo di un comitato costituito ad hoc o di un consiglio aziendale ${ }^{9}$.

Assume particolare rilevanza la previsione della partecipazione diretta dei lavoratori, in tal modo interpellati dal datore di lavoro anticipatamente rispetto all'adempimento di importanti obblighi, nelle imprese ove sia assente una formale rappresentanza specializzata. Del resto la «consultazione e partecipazione dei lavoratori», in aggiunta o in alternativa a quella dei loro rappresentanti, fa parte dei principi racchiusi nella direttiva quadro 89/391 all'art. $11^{10}$.

7 Lord Robens, Safety and Health at Work. Report of the Committee 197072, cit. Al riguardo v. B. BARRETT, R. Howells, Occupational health and safety law: texts and materials, London, Cavendish Publishing, 2000, p. 14 ss.

8 D. Walters, E. Wadsworth, The United Kingdom, in D. Walters, E. Wadsworth, M. QuinLan, Analysis of the determinants of workplace occupational safety and health practice in a selection of EU Member States, Cardiff Work Environment Research Centre Cardiff University, 2012, p. 117.

9 Cfr. sul tema E. Ales, Occupational Health and Safety: a European and Comparative Legal Perspective, cit., p. 36 ss.; nonché V. Howes, Workers' Involvement in Health and Safety Management and Beyond: The UK Case, in The International Journal of Comparative Labour Law and Industrial Relations, 2007, p. 249.

10 Cfr. R. Del Punta, La rappresentanza sindacale e le rappresentanze dei lavoratori nel quadro degli sviluppi del diritto comunitario, in DRI, 1993, p. 193, il quale ha precisato come «l'idea» sottesa alla direttiva 89/391 è quella di una «par- 
Per meglio chiarire questo aspetto si può richiamare l'esempio del Regno Unito, che nelle Health and Safety (Consultation with Employees) Regulations 1996 ha contemplato al paragrafo 3 il dovere datoriale di consultare i lavoratori, qualora non siano rappresentati da Safety Representatives, su decisioni che attengono all'introduzione di nuove tecnologie con possibili conseguenze sulla loro salute, nonché all'organizzazione delle attività preventive ${ }^{11}$.

Ma anche nella Ley de Prevención de Riesgos Laborales del 1995, propria del contesto spagnolo, è espressamente regolata la «consulta de los trabajadores», quale alternativa a quella dei rappresentanti dei lavoratori, in merito alla pianificazione e organizzazione del lavoro nell'impresa e delle misure di protezione della salute e a qualsiasi altra azione che possa avere effetti sostanziali sulle condizioni di sicurezza ${ }^{12}$.

Nell'ordinamento interno la "partecipazione e consultazione dei lavoratori» figura tra le misure generali di tutela (art. 15, comma 1, lett. r, d.lgs. n. 81/2008), mentre più specificamente nella Sezione VII il riferimento è solo alla consultazione e partecipazione dei rappresentanti dei lavoratori, che come già chiarito risultano i "protagonisti", nonostante il ruolo attivo riconosciuto ai prestatori dallo stesso decreto. Con lo sguardo della comparazione la dimensione partecipativa assunta dai singoli creditori di sicurezza pare, in sostanza, sminuita.

Si deve, infine, riflettere sulla natura obbligatoria o volontaria delle forme di partecipazione collettiva menzionate e sulle modalità della loro costituzione. Più esattamente non è di poco conto, al fine di una riflessione comparata, l'incertezza che ha contraddistinto il modello di rappresentanza sviluppato nell'ordinamento interno, tuttora definibile in un certo senso di tipo volontario, in conside-

tecipazione diffusa che coinvolga tutti i lavoratori nei problemi concernenti la loro salute».

11 Si rinvia sul punto al paragrafo 2 .

12 Si tratta dell'art. 33 della Ley de Prevención de Riesgos Laborales, inserito nel capitolo V, dedicato alla «consulta y participación de los trabajadores». In proposito cfr. J. Romeral Hernández, Consulta y participación de los trabajadores, in A.V. Sempere Navarro (dir.), M. Cardenal Carro, I. Alzaga Ruiz (coord.), Comentarios a la Ley de Prevención de Riesgos Laborales, cit., p. 660 ss. 
razione dell'affidamento del mandato rappresentativo all'iniziativa dei lavoratori, nonché dei poteri riconosciuti all'autonomia collettiva in merito all'esatta individuazione delle modalità di costituzio$\mathrm{ne}^{13}$. L'eventuale assenza a livello aziendale del rappresentante dei lavoratori per la sicurezza è stata, appunto, immaginata ed espressamente regolata attraverso la previsione nel d.lgs. n. 81/2008 del rappresentante territoriale (art. 48) e del rappresentante di sito produttivo (art. 49), deputati a svolgere le relative funzioni assegnate al RLS. Ciò non contraddice, tuttavia, la «natura necessaria e legale» della rappresentanza per la sicurezza di cui al d.lgs. n. 81/2008, «tanto per i lavoratori quanto per lo stesso sistema di prevenzione aziendale» ${ }^{14}$.

Diversamente, in altri Paesi l'istituzione di specifiche rappresentanze dei lavoratori o di appositi organismi congiunti è prevista come obbligatoria ed il relativo procedimento è rimesso all'iniziativa del $m a$ nagement, con non poche conseguenze in caso di omissione. A mo' di esempio, vige in Francia un obbligo in capo al datore di lavoro, sanzionabile civilmente e penalmente, di organizzare l'elezione della delegazione del personale che comporrà il Comité d'hygiène, de sécurité et des conditions de travail. Le conseguenze dell'inadempimento datoriale possono essere considerate particolarmente gravi, posto che l'istituzione del Comité è una misura fondamentale nei meccanismi di prevenzione ${ }^{15}$. Ne deriva un modello di rappresentanza obbligatorio, ancorché nelle imprese di maggiori dimensioni.

13 P. Campanella, I rappresentanti dei lavoratori per la sicurezza, in L. Zoppoli, P. Pascucci, G. Natullo (a cura di), Le nuove regole per la salute e la sicurezza dei lavoratori, cit., p. 482; nonché EAD., Profili collettivi di tutela della salute e rappresentanza dei lavoratori per la sicurezza: disciplina legislativa, bilancio applicativo, prospettive di riforma, cit., p. 156.

${ }_{14}$ P. PAscucci, Salute e sicurezza: dalle rappresentanze dell'art. 9 ai rappresentanti del d.lgs. n. 81/08, cit., p. 668 , il quale ha precisato che «accanto ad una dimensione pubblicistico-obbligatoria», relativa alla presenza e alle funzioni del rappresentante dei lavoratori per la sicurezza, emergono anche «elementi di volontarietà» quanto in particolare al «quomodo della sua individuazione».

15 G. Loiseau, L. Pécaut-Rivolier, P.-Y. Verkindt, Le Guide du Comité d'hygiène, de sécurité et des conditions de travail (CHSCT), Paris, Dalloz, 2015, p. 123 ss., ove è chiarito che «l'assenza dell'istituzione di un Comité d'hygiène, de sécurité et des conditions de travail potrebbe essere considerata come una violazione diretta del datore di lavoro al suo obbligo di sicurezza». 
2. Il dovere di consultazione dei rappresentanti dei lavoratori nel Regno Unito e l'ipotesi del Safety Committee

Una disciplina peculiare in materia di rappresentanze dei lavoratori per la sicurezza, sempre se comparata con le regole interne al nostro ordinamento, è quella propria del Regno Unito, ove l'Health and Safety at Work Act (HSWA) impone nella section 2.6, nell'ambito dei doveri generali in capo al datore di lavoro, di consultare i rappresentanti dei lavoratori nominati da organizzazioni sindacali riconosciute. La finalità è quella di promuovere misure volte a garantire la salute sul lavoro attraverso la cooperazione tra le parti (datore di lavoro e lavoratori).

Precise indicazioni, al riguardo, sono rinvenibili nelle Safety Representatives and Safety Committees Regulations 1977, che in origine regolavano l'unica forma di partecipazione dei lavoratori in materia, riservata anche ora agli ambienti di lavoro ove sono presenti organizzazioni sindacali riconosciute ${ }^{16}$.

Anzitutto, la disciplina del 1977 non indica quanti rappresentanti debbano esservi all'interno dell'azienda, ma affida tale individuazione ad un accordo tra datore di lavoro e sindacati.

Come chiarito dall'Health and Safety Executive (HSE) i Safety Representatives sono indipendenti dal management aziendale, in quanto espressione dei lavoratori e legati ad essi da un vincolo fiduciario. Le loro funzioni sono quelle di rappresentarne gli interessi in materia di «health, safety and welfare» ${ }^{17}$. Tra i poteri loro riconosciuti v'è da segnalare quello di indagare sulle cause degli infortuni, sui reclami dei lavoratori e sulle situazioni pericolose, di ispezionare i luoghi di lavoro, di rappresentare i lavoratori di fronte agli ispettori dell'HSE.

L'obbligo di consultazione a carico del datore di lavoro ricorre in ogni caso di cambiamento che possa sostanzialmente riguardare la salute e la sicurezza, come ad esempio l'introduzione di nuove o differenti procedure, tipologie di lavoro o attrezzature.

16 Cfr. M. Bell, Occupational Health and Safety in the UK: At a Crossroads?, in E. Ales (ed.), Health and Safety at Work. European and Comparative Perspective, cit., p. 396. Per una riflessione sul tema cfr. A. Lo FAro, Azione collettiva e tutela dell'ambiente di lavoro in Europa, in DLRI, 1991, p. 170 ss.

17 Così, esattamente, secondo il paragrafo 4, lettera d, delle Safety Representatives and Safety Committees Regulations 1977. 
Assume, inoltre, importanza la facoltà riconosciuta ad almeno due Safety Representatives di richiedere al datore di lavoro l'introduzione di un Safety Committee. Tale Comitato, secondo le indicazioni del HSWA, section 2.7, ha, in primo luogo, la funzione di controllare le misure adottate per assicurare la protezione dei lavoratori.

In mancanza di previsioni in merito al numero dei suoi componenti, che possono variare in funzione delle dimensioni dell'azienda e delle sue articolazioni, le indicazioni fornite dall'HSE chiariscono che si tratta di un organo misto, generalmente formato da rappresentanti del management aziendale e dei lavoratori (sia di nomina sindacale, sia eletti), oltreché da altri soggetti presenti nell'ambiente di lavoro e con competenze specifiche in materia (company doctor, health and safety adviser). Non si è mancato, in proposito, di suggerire che vi dovrebbe essere un equilibrio tra i componenti all'interno del Safety Committee, escludendo così che i rappresentanti dei lavoratori siano inferiori numericamente rispetto ai rappresentanti del management ${ }^{18}$.

Ad integrazione delle scarne disposizioni legislative assume rilevanza l'Approved Code of Practice and guidance, intitolato «Consulting workers on health and safety. Safety Representatives and Safety Committees Regulations» del 2014, che specifica le funzioni che possono essere svolte dal Comitato ossia, tra le altre, lo studio degli infortuni sul lavoro, con l'esplicito intento di impedirne il ripetersi, e la consultazione sulle regole e misure di prevenzione e sull'effettività della formazione per i lavoratori ${ }^{19}$. In sostanza, il Safety Committee ha il ruolo di offrire consigli sulla adeguatezza delle regole di prevenzione proposte dal datore di lavoro e di segnalare la necessità di intervenire in ipotesi di attività particolarmente rischiose.

L'azione del Comitato è riassumibile nella discussione sulla necessità di misure prevenzionistiche, nelle raccomandazioni ed, infine,

18 V. le osservazioni al riguardo di D. KLoss, Occupational Health Law, Oxford, Wiley-Blackwell, 2010, p. 157 ss.

19 L'Approved Code of Practice and guidance, «Consulting workers on health and safety. Safety Representatives and Safety Committees Regulations», p. 36, rimarca che l'indagine svolta dal Comitato sugli infortuni sul lavoro deve essere volta a «look at the facts in an impartial way», «consider what sort of precautions might be taken», «make appropriate recommendations». 
nella revisione e controllo del buon esito delle iniziative intraprese. Il verbale delle riunioni è, altresì, reso accessibile a tutti i lavoratori.

Si può, allora, ritenere, anche sulla scorta di alcuni documenti pubblicati proprio dall'HSE ove è messo in luce il ruolo determinante del coinvolgimento dei prestatori di lavoro, che la presenza del Comitato sia un efficace strumento per migliorare i livelli di sicurezza e le condizioni lavorative ${ }^{20}$.

È anche vero che in base alle Safety Representatives and Safety Committees Regulations 1977, come sottolineato in dottrina, una grande percentuale della forza lavoro è stata esclusa dal coinvolgimento in materia di salute e sicurezza almeno fino al 1996, quando questa regolamentazione è stata affiancata dalle Health and Safety (Consultation with Employees) Regulations 1996; era infatti, apparso chiaro che il Regno Unito non aveva pienamente dato attuazione all'art. 11 della direttiva quadro 89/391/CEE, «escludendo le imprese non sindacalizzate» ${ }^{21}$. Da questo momento in poi è stato rilevato un incremento nel coinvolgimento e nella consultazione in materia di salute e sicurezza sul lavoro ${ }^{22}$.

Le Health and Safety (Consultation with Employees) Regulations 1996 si applicano, appunto, in presenza di lavoratori che non hanno una rappresentanza nominata da «recognised Trade unions».

20 Si veda D. Walters, T. Nichols, J. Connor, A.C. Tarisan, S. CAm, The role and effectiveness of safety representatives in influencing workplace health and safety, Health and safety executive, 2005, p. xiii, in www.hse.gov.uk., ove è sottolineato che «le rappresentanze dei lavoratori e la loro consultazione hanno un significativo ruolo nell'elevare la salute e sicurezza sul lavoro». Cfr., inoltre, Improving worker involvement - Improving health and safety, 2006, in www.hse.gov.uk/consult/index/htm.

21 V. Howes, Workers' Involvement in Health and Safety Management and Beyond: The UK Case, cit., p. 257, che ha precisato come le Health and Safety (Consultation with Employees) Regulations 1996 hanno costituito la risposta diretta alla direttiva 89/391/CEE. Sull'importanza della direttiva quadro con riferimento al problema della rappresentanza dei lavoratori in materia cfr. A.C. NEAL, The European Framework Directive on the Health and Safety of Workers: Challenges for the United Kingdom?, in The International Journal of Comparative Labour Law and Industrial Relations, 1990, vol. 6, p. 95. Cfr., inoltre, la pronuncia della Corte di Giustizia Europea 8 giugno 1994, Commission of the European Communities v. United Kingdom of Great Britain and Northern Ireland, n. C-383/92, che ha stabilito la contrarietà al diritto europeo di una normativa nazionale che preveda la consultazione dei lavoratori escludendo alcuni di essi.

22 Cfr. S. Deakin, G.S. Morris, Labour Law, cit., p. 952. 
La consultazione da parte del datore di lavoro, in tal caso, può essere diretta ed individuale, ipotesi probabilmente percorribile solo nelle imprese di piccole dimensioni, o avvenire attraverso rappresentanti appositamente eletti dai lavoratori ${ }^{23}$. Queste rappresentanze non sono del tutto assimilabili ai Safety Representatives, in quanto prive del potere di indagine sulle cause degli incidenti e sui potenziali rischi e pericoli, nonché del potere di ispezione e, soprattutto, della facoltà di richiedere la costituzione di un Safety Committee.

Da questo punto di vista, in dottrina è stata avanzata la proposta di equiparare le funzioni di entrambe le rappresentanze dei lavoratori, che siano nominate o meno dalle organizzazioni sindacali ${ }^{24}$.

Infine, in ordine alle conseguenze in caso di violazione dell'obbligo di consultazione da parte del datore di lavoro, è previsto il potere in capo agli ispettori di emettere un «improvement notice» ossia un ordine di porre in essere un rimedio entro un termine stabilito; nell'ipotesi ulteriore del mancato rispetto di tale ordine, la conseguenza è un «criminal offence» ${ }^{25}$.

\section{Il Comité d'hygiène, de sécurité et des conditions de travail}

Come anticipato, destano peculiare interesse gli organismi definiti misti che, presenti in diversi paesi europei, corrispondono a istituti di rappresentanza dei lavoratori e contribuiscono alla promozione della partecipazione nella tutela della salute sul lavoro.

${ }^{23}$ Si veda, in particolare, il paragrafo 5 delle Health and Safety (Consultation with Employees) Regulations 1996, che impone al datore di lavoro di fornire ai lavoratori o ai rappresentanti eletti le informazioni necessarie per permettere loro di partecipare in modo completo ed efficace alla consultazione. In merito ad una riflessione sul ruolo delle rappresentanze dei lavoratori nelle piccole imprese cfr. D. WALTERS, Employee representation and health and safety: A strategy for improving health and safety performance in small enterprises?, in Employee Relations, 1998, vol. 20, p. 180.

24 M. Bell, Occupational Health and Safety in the UK: At a Crossroads?, in E. Ales (ed.), Health and Safety at Work. European and Comparative Perspective, cit., p. 398.

25 S. Deakin, G.S. Morris, Labour Law, cit., 2012, p. 953. Si veda, in particolare, l'Health and Safety Executive Enforcement Policy Statement, ottobre 2015, che enuncia le politiche seguite nella verifica degli adempimenti in materia di salute e sicurezza sul lavoro. 
Esplicite previsioni legislative sono quelle contenute nel Code $d u$ travail $^{26}$ francese in merito alla costituzione, ai compiti e funzioni del Comité d'hygiène, de sécurité et des conditions de travail $(C H S C T)$, la cui rilevanza, se non centralità, è stata ampiamente sottolineata in dottrina ${ }^{27}$.

Organismo debole fino agli anni ottanta, ha conosciuto da allora uno sviluppo continuo al punto di essere definito oggi come protagonista necessario («acteur incontournable»); il CHSCT si offre, in un certo senso, come contropotere rispetto alle iniziative manageriali suscettibili di compromettere la salute, in particolare mentale, dei lavoratori ${ }^{28}$.

Il CHSCT si distingue, anzitutto, dal Comité d'entreprise, inteso come «il perno del coinvolgimento dei lavoratori nell'ordinamento francese $»^{29}$, trattandosi di un organismo rappresentativo specializzato, i cui componenti provengono dalla collettività dei lavoratori dell'impresa ${ }^{30}$.

Un istituto obbligatorio nelle imprese con almeno cinquanta dipendenti ed anche in quelle di minori dimensioni ove lo richieda l'ispettore del lavoro, a causa della natura del lavoro, della struttura

26 Quanto alle più recenti modifiche al Code du travail cfr. la Loi relative au travail, à la modernisation du dialogue social et à la sécurisation des parcours professionnels $n$. 2016-1088. Si veda, altresì, il progetto di Ordonnance relative à la nouvelle organisation du dialogue social et économique dans l'entreprise et favorisant l'exercice et la valorisation des responsabilités syndicales, presentato il 31 agosto 2017, ove è contenuta la «fusion des institutions représentatives du personnel».

27 Sottolineano la crescita di importanza dell'istituto, in particolare, S. GuEDES Da Costa, E. Lafuma, Le CHSCT dans la décision d'organisation du travail, in Revue de droit du travail, 2010, p. 419 ss., nonché C. Wolmark, Comité d'hygiène, de sécurité et des conditions de travail, in P. Zawieja, F. GuARNIERI (sous la direction de), Dictionnaire des risques psychosociaux, Paris, Le Seuil, 2014, p. 114 ss., che ne ripercorre l'origine storica.

28 C. Wolmark, Comité d'hygiène, de sécurité et des conditions de travail, cit., p. 115, il quale precisa che il CHSCT è divenuto attraverso la Loi n. 82-1097 del 23 dicembre del 1982 una «vera istituzione di rappresentanza dei lavoratori»e non è più, come in passato, un «semplice organismo tecnico d'appoggio» per la realizzazione di una politica di sicurezza essenzialmente datoriale.

29 M. CorTI, La partecipazione dei lavoratori. La cornice europea e l'esperienza comparata, Milano, Vita e Pensiero, 2012, p. 209.

30 P. LoKIEc, Droit du travail. Les relations collectives de travail, Paris, Presses Universitaires de France, 2011, p. 117 ss. 
o delle attrezzature dei locali aziendali (art. L. 4611-4 Code du travail); sostanzialmente in ragione di un'organizzazione del lavoro reputata alquanto pericolosa ${ }^{31}$.

Quale «institution de proximité» è anche previsto nell'ambito dei singoli stabilimenti dell'azienda (che impieghino ciascuno almeno cinquanta lavoratori), al fine di consentire ai rappresentanti presenti nel Comité di essere vicini al luogo di lavoro ${ }^{32}$. In sua assenza, le relative funzioni sono espressamente assegnate ai délégués du personnel, che esercitano i diritti di rappresentanza e di coinvolgimento dei prestatori di lavoro in generale ${ }^{33}$.

Come già sottolineato, si configura come un organismo misto, composto dal datore di lavoro e da una delegazione eletta del personale il cui numero varia in funzione dei lavoratori assunti (art. L 4613-1). Per la verità, la legge è poco chiara ed esplicativa in merito alle condizioni di designazione dei componenti del CHSCT; si precisa unicamente che essi devono essere designati da un collegio che riunisce i componenti eletti del Comité d'entreprise e i delegati del personale ${ }^{34}$. I componenti del CHSCT sono, dunque, scelti tra i lavoratori eleggibili, i quali dovranno ricevere

31 Si deve ricordare che, secondo le disposizioni del Code du travail, le imprese con meno di cinquanta lavoratori possono raggrupparsi per costituire un $C H S C T$ (art. L 4611-6); la legge, tuttavia, non fornisce precisazioni sulle modalità di istituzione o sulle attribuzioni di questo comitato tra più imprese («interentreprises»).

32 P. LoKIEc, Droit du travail, cit., p. 118. Precisamente ai sensi dell'art. L 4611-1 «l'istituzione di un comitato è necessaria solo se è stato raggiunto il numero di almeno cinquanta dipendenti per dodici mesi consecutivi o non consecutivi nel corso dei tre anni precedenti».

33 Il citato progetto di Ordonnance relative à la nouvelle organisation du dialogue social et économique dans l'entreprise et favorisant l'exercice et la valorisation des responsabilités syndicales contempla, tuttavia, l'attribuzione all'unico Comité Social et Economique (CSE), nell'ambito delle imprese con almeno cinquanta dipendenti, delle funzioni esercitate dai délégués du personnel, dal Comité d'entreprise, e dal Comité d'hygiène, de sécurité et des conditions de travail.

34 Come chiarito in dottrina e in giurisprudenza la designazione dei componenti del CHSCT si svolge sotto forma di elezione. Una volta ravvisate le condizioni per la sua istituzione, il datore di lavoro ha l'obbligo, sanzionabile civilmente e penalmente (art. L 4742-1), di istituire un CHSCT organizzando l'elezione della delegazione del personale che comporrà il Comité: in proposito cfr. G. LOISEAU, L. Pécaut-Rivolier, P.-Y. Verkindt, Le Guide du Comité d'hygiène, de sécurité et des conditions de travail (CHSCT), cit., p. 122 ss.; in giurisprudenza cfr., in particolare, Cass. soc. 17 mai 2011, n. 10-12.852, in Bull. civ., 2011, V, n. 108. 
una formazione «necessaria all'esercizio delle loro funzioni» (art. L 4614-14).

Nelle imprese di ampie dimensioni il numero dei CHSCT presenti è fissato per accordo tra il datore di lavoro e il Comité d'entreprise, in considerazione della natura e gravità dei rischi e delle modalità organizzative del lavoro; in ipotesi di disaccordo, invece, per decisione amministrativa (art. L 4613-4) ${ }^{35}$.

Il CHSCT è, appunto, ormai identificato come una istituzione rappresentativa del personale, a causa soprattutto della redistribuzione dei ruoli fra i differenti suoi componenti. Il datore di lavoro, anche se presidente, è escluso dal voto di numerose risoluzioni (art. L 4614-1) ed i rappresentanti dei lavoratori pare costituiscano la parte decisamente predominante ${ }^{36}$. Questa «democratizzazione» della composizione del CHSCT è, del resto, progredita con l'estensione dell'obbligo della sua istituzione ${ }^{37}$.

È stato chiarito che la valenza di un CHSCT «dipende dalla presenza sindacale e dal rapporto di forze ottenuto dai lavoratori nello stabilimento, come anche dal livello dei rischi legati al lavoro» ${ }^{38}$. In verità, è alquanto incerto e dibattuto il ruolo delle organizzazioni sindacali nella istituzione del Comité, considerato che il Code du travail non accorda loro alcun privilegio nella partecipazione alla sua elezione ${ }^{39}$. Occorre, tuttavia, ricordare che già l'accord cadre interprofessionnel du 17 mars 1975 sur l'amélioration des conditions de travail, al fine di un maggior coinvolgimento delle organizzazio-

35 Da tempo si discute in dottrina in merito all'opportunità di introdurre un CHSCT centrale che coesista con i diversi comitati presenti negli stabilimenti, in modo simile a quanto previsto per i Comitati d'impresa. Cfr., al riguardo, le considerazioni di P.-Y. VERKINDT, Quand les conditions de travail s'invitent dans la sécurisation de l'emploi, in Droit Social, 2013, p. 730 ss.

36 C. Wolmark, Comité d'hygiène, de sécurité et des conditions de travail, cit., p. 115.

37 C. Wolmark, Comité d'hygiène, de sécurité et des conditions de travail, cit., p. 116, il quale riferisce come nel settore privato, secondo le ultime cifre disponibili (2004/2005), il 72\% delle imprese soggette all'obbligo di istituirlo sono dotate di un CHSCT, il che rappresenta circa 25.000 organismi.

38 T. Coutrot, Le rôle des comités d'hygiène, de sécurité et des conditions de travail en France, in Travail et emploi, 2009, n. 117, p. 25 ss.

39 G. Loiseau, L. Pécaut-Rivolier, P.-Y. Verkindt, Le Guide du Comité d'hygiène, de sécurité et des conditions de travail (CHSCT), cit., p. 220. 
ni sindacali, ha previsto la possibilità che ciascuna organizzazione designi, negli stabilimenti che impiegano più di 300 lavoratori, un rappresentante che assisterà con voce consultiva alle riunioni del $\mathrm{CHSCT}^{40}$. Inoltre, i contratti collettivi possono ugualmente prevedere questa stessa facoltà ai sensi dell'art. L 4611-7, che ammette l'introduzione ad opera della contrattazione collettiva di disposizioni più favorevoli in merito al funzionamento, alla composizione ed ai poteri del Comitét $^{41}$.

La rilevanza attribuita all'istituto emerge, in particolare, dai compiti affidatigli dal Code du travail ossia di contribuire «alla protezione della salute fisica e mentale e della sicurezza dei lavoratori», «al miglioramento delle condizioni di lavoro», nonché di «garantire l'osservanza delle prescrizioni legali» in questa materia (art. L 4612$1)^{42}$.

Il CHSCT è chiamato a giocare, dunque, «un ruolo importante» ${ }^{43}$ nella prevenzione dei rischi sul lavoro, posto che deve partecipare all'elaborazione del piano di prevenzione (obbligatorio in tutte le imprese) e, soprattutto, deve essere consultato dal datore di lavoro prima di ogni cambiamento tecnico o organizzativo, da cui derivino conseguenze sulle condizioni di lavoro. In ragione di ciò non si può quindi trascurare, ai fini della presente ricerca, di prendere in esame tale organismo, riflettendo soprattutto sulla possibilità ad esso conferita di proporre misure prevenzionistiche migliorative e, conseguentemente, di incidere sulle decisioni datoriali.

Il CHSCT è, anzitutto, un attore della prevenzione: procede all'analisi dei rischi professionali e delle condizioni di lavoro (art. L 4612-2), contribuisce alla promozione della prevenzione dei rischi e promuove ogni iniziativa utile in tale direzione, con particolare ri-

40 In merito alla questione della presenza sindacale all'interno del CHSCT cfr., in particolare, le considerazioni di P.-Y. VERKINDT, Le représentant syndical au comité d'hygiène, de sécurité et des conditions de travail, in Droit Social, 2009, n. 2, p. 181 ss.

41 G. Loiseau, L. Pécaut-Rivolier, P.-Y. Verkindt, Le Guide du Comité d'hygiène, de sécurité et des conditions de travail (CHSCT), cit., p. 221.

42 Il riferimento alla tutela della salute mentale consegue alla Loi de modernisation sociale (73/2002 del 17 gennaio 2002).

43 T. Coutrot, Le rôle des comités d'hygiène, de sécurité et des conditions de travail en France, cit., p. 27. 
ferimento ad azioni di prevenzione delle molestie morali e sessuali (art. L 4612-3); è, inoltre, consultato prima di ogni decisione di organizzazione (décision d'amenagement) importante che modifichi le condizioni di salute e di sicurezza o le condizioni di lavoro (art. L 4612-8-1), nonché in merito al progetto di introduzione di nuove tecnologie ed alle possibili conseguenze (art. L 4612-9).

La dottrina ha fornito peculiare risalto alla citata obbligatoria consultazione del CHSCT prima di ogni décision d'amenagement importante ${ }^{44}$, da cui derivino conseguenze pregiudizievoli per i prestatori di lavoro e, in special modo, «prima di ogni trasformazione importante dei posti di lavoro», conseguente alla «modifica dell'attrezzatura o dell'organizzazione del lavoro», nonché prima di ogni cambiamento dei tempi e delle regole di produzione (art. L 46128-1 $)^{45}$.

Al di là delle previsioni di consultazioni obbligatorie, il CHSCT si può pronunciare su ogni questione di cui sia stato incaricato dal datore di lavoro, dai delegati del personale e dal Comité d'entreprise; quest'ultimo può, infatti, affidare al CHSCT studi riguardanti materie di sua competenza e interpellarlo, quale istituto specializzato, su questioni inerenti all'organizzazione lavorativa, alla tecnologia, alle condizioni d'impiego, all'orario e alla remunerazione del lavoro, che possano incidere sulla salute dei lavoratori (art. L 2323-16) ${ }^{46}$.

Accade, altresì, che il Comité d'entreprise e il CHSCT, entrambi istituti di rappresentanza (da un lato generale e dall'altro specifica), siano chiamati a pronunciarsi congiuntamente sulle medesime problematiche ${ }^{47}$. Invero, la determinazione collettiva delle condizioni di lavoro rientra in un certo senso nel campo di azione e nell'ambito

44 Si veda E. Peskine, C. Wolmark, Droit du travail, cit., p. 606, ove questa espressione è definita «aperta».

45 La giurisprudenza ha rimarcato l'elasticità delle nozioni utilizzate dal legislatore come «conditions de travail», «conditions de santé et sécurité» e ha esteso il campo di intervento dell'istituto: cfr. Cass. soc. 29 sept. 2009, n. 08-17023.

46 Sulle modalità di formulazione del parere del CHSCT cfr., in particolare, in giurisprudenza: Cass. soc. 10 janv. 2012, n. 10-23.206, in Droit Social, 2012, p. 318, con commento di F. PetrT. In merito all'ipotesi di rifiuto di parere da parte del CHSCT cfr. la circulaire Direction générale du travail, 2014/1, 18 mars 2014.

47 La doppia consultazione di Comité d'entreprise e di CHSCT è prevista, ad esempio, in merito al progetto di introduzione di nuove tecnologie e alle conseguenze di tale progetto (L 4612-9 e L 2323-29). 
delle competenze del CHSCT, oltreché del Comité d'entreprise, con il rischio da alcuni paventato di una invasione del CHSCT nelle prerogative riconosciute al Comité d'entreprise ${ }^{48}$. Quali, dunque, i confini rispetto al campo di intervento dei due istituti, vicini quanto alla struttura e alle modalità di funzionamento ${ }^{49}$ ?

La dottrina si è spesso posta questo interrogativo, in virtù anche della valorizzazione del Comité d'entreprise e dell'incremento delle modalità di esercizio delle sue funzioni successivamente alla Loi $n$. 504/2013 relative à la sécurité de l'emploi du 14 juin $2013^{50}$.

Nelle richiamate circostanze il CHSCT, avendo una funzione chiarificatrice, deve essere consultato ed emettere il suo parere prima che il Comité d'entreprise renda il proprio; verificandosi, in caso contrario, l'irregolarità della procedura ${ }^{51}$. Vale, poi, per il CHSCT, quanto previsto per il Comité d'entreprise, ossia la necessità di disporre delle informazioni necessarie al fine dell'esercizio delle proprie prerogative (art. L 4614-9) ${ }^{52}$.

Come statuito nelle decisioni giurisprudenziali, ha assunto rilievo la richiesta di parere preventivo del CHSCT anche nell'ipotesi di realizzazione da parte del datore di lavoro di un sistema di valutazione del lavoro dei dipendenti, in considerazione dell'incidenza sul loro comportamento e delle possibili conseguenze sulla loro salute; ne deriva che ogni decisione del datore di lavoro che possa generare «inquietudine» per i lavoratori deve essere sottoposta al vaglio del $C H S C T^{53}$.

48 In tal senso S. Guedes da Costa, E. Lafuma, Le CHSCT dans la décision d'organisation du travail, cit., p. 419; in proposito cfr., inoltre, J. Pélissier, G. Auzero, E. Dockés, Droit du travail, cit., p. 883.

49 Sottolinea questa vicinanza tra Comité d'entreprise e CHSCT A. MAzEAUd, Droit du travail, cit., p. 146.

50 G. Borenfreund, Le comité d'entreprise: nouveaux enjeux, in Revue de droit du travail, 2015, p. 17. In proposito cfr. le considerazioni di M. CorTI, Le nuove frontiere della partecipazione dei lavoratori in Francia, in DRI, 2014, p. 284, secondo il quale i diritti di informazione e consultazione del Comité d'entreprise "già estremamente articolati, ne escono ulteriormente irrobustiti».

51 In tal senso v. Cour d'appel de Paris 3 nov. 2006; in proposito cfr. le riflessioni di B. Teyssié, Droit du travail. Relations collectives, Paris, Lexis Nexis, 2014, p. 418.

52 Il datore di lavoro è, inoltre, tenuto a fornire al CHSCT annualmente un bilancio della situazione generale sulle condizioni di lavoro e un programma di prevenzione dei rischi professionali e di miglioramento delle condizioni di lavoro (art. L 4612-16).

53 Si richiama, in particolare, Cass. soc. 28 nov. 2007, che si è pronunciata sulla necessità che i criteri della valutazione annuale del datore di lavoro siano pre- 
Tale potere prevalentemente consultivo assume valore decisivo al punto che il Comité d'hygiène, de sécurité et des conditions de travail, quale espressione degli interessi dei lavoratori, è considerato il «luogo essenziale di regolazione al fine di rendere effettive le politiche di prevenzione in materia di igiene, sicurezza, salute fisica e mentale dei lavoratori» ${ }^{4}$. Dunque, uno «spazio di discussione» e di incontro tra «saperi» diversi ove sorgono eventuali azioni di miglioramento della sicurezza nei luoghi di lavoro ${ }^{55}$, realizzandosi un dialogo proficuo tra rappresentanti dei lavoratori e controparte datoriale.

Ma il CHSCT ha, secondo le previsioni del Code du travail, anche il potere di reagire a fronte di pericoli effettivi per la salute dei lavoratori, realizzando ad esempio inchieste in materia di infortuni e malattie professionali (art. L 4612-5), presentando osservazioni in occasione delle visite da parte dell'ispettore del lavoro (art. L 4212 7), e finanche ricorrendo ad un'expertise in ipotesi di rischio grave all'interno dello stabilimento o di progetto importante che modifichi le condizioni di salute e sicurezza o le condizioni di lavoro, come previsto dal citato art. L 4612-8-156.

Per una completa e precisa comprensione delle problematiche attinenti alla salute e sicurezza, il CHSCT dispone, inoltre, della facoltà di effettuare delle ispezioni periodiche, che gli consentono di controllare la corretta applicazione delle regole, come imposto dall'art. L 4612-4, nonché di predisporre delle riunioni, di cui all'art. L 4614-7, cui possono partecipare il medico del lavoro e il responsabile della sicurezza nello stabilimento.

In ipotesi di inadempimento datoriale all'obbligo di consultazio-

ventivamente sottoposti al giudizio del CHSCT. Sui poteri di intervento del CHSCT vedi, inoltre, Cass. soc. 5 mars 2008. Cfr. L. Lerouge, Le rôle du CHSCT reconnu par la jurisprudence, in Santé \& Travail, 2015, p. 29.

54 Cfr. Les CHSCT, entre dispositifs et pratiques, Anact, Janvier, 2012, p. 16.

55 L. Lerouge, P.-Y. Verkindt, Sauvegarder et renforcer le CHSCT: un enjeu majeur de santé au travail, in Droit Social, 2015, p. 365, ove sono messi in luce gli aspetti positivi legati al CHSCT e si sottolinea la necessità della sua presenza anche in imprese di piccole dimensioni.

56 Rileva, inoltre, il «diritto di allerta» esercitabile da parte del rappresentante del personale nel CHSCT informato di un pericolo grave e imminente (art. L 4131-2). 
ne e confronto con il CHSCT, è integrato il «délit d'entrave» e residua la possibilità di rimettere al giudice la questione della sospensione della decisione finale e la riparazione del conseguente pregiudizio $^{57}$. All'assenza di consultazione la giurisprudenza ha, poi, assimilato l'insufficienza dell'informazione fornita da parte del datore di lavoro, che impedisca al CHSCT di formulare utilmente il suo parere. Nonostante la diversa gravità delle due ipotesi, le conseguenze sarebbero, allora, le stesse, considerato che l'insufficienza dell'una vizia la regolarità dell'altra ${ }^{58}$.

Ulteriore problematica concerne, poi, l'eventualità che il datore di lavoro non tenga in debito conto l'esito del coinvolgimento del CHSCT: un suo parere negativo non può, infatti, ostacolare l'iniziativa datoriale. Le proposte del CHSCT non possono, però, essere respinte sic et simpliciter, dovendo il datore di lavoro motivare ed esplicitare le ragioni che lo spingono a rifiutare (art. L 4612-3).

Si può conclusivamente sostenere che il CHSCT, pur privo di potere decisionale, rappresenta quantomeno il luogo di creazione di una «concezione concorrente» o «alternativa» rispetto a quella manageriale, tanto più forte quanto più lo stesso organismo possa contare su informazioni adeguate; rappresenta, altresì, il «crocevia» tra i differenti punti di vista di lavoratori e datore di lavoro ${ }^{59}$. Per poter realmente influire sulle decisioni datoriali un ruolo decisivo assumono, inoltre, le riunioni che il Comitato ha l'obbligo di tenere ogni trimestre, specialmente nei settori di attività che presentano rischi particolari.

$\mathrm{Va}$, quindi, ribadita la peculiarità di questa forma di coinvolgimento, del tutto assente nell'ordinamento italiano, ad eccezione dell'introduzione di commissioni analoghe per via contrattuale come si è già considerato. Si può, di nuovo rimarcare come il CHSCT abbia acquisito negli orientamenti dottrinali e giurisprudenziali un

57 In giurisprudenza v. Cass. soc. 30 juin 2010, n. 09-13.640, in Droit Social, 2010, p. 1006, con commento di L. Pécaut-Rivolier.

58 Cass. soc. 25 sept. 2013, n. 12-21.747, in Bull. civ., 2013, V, n. 211; sul punto cfr. G. Loiseau, L. Pécaut-Rivolier, P.-Y. Verkindt, Le Guide du Comité d'hygiène, de sécurité et des conditions de travail (CHSCT), cit., p. 329.

59 C. Wolmark, Comité d'hygiène, de sécurité et des conditions de travail, cit., p. 119. 
ruolo particolarmente significativo quanto alla prevenzione dei rischi, anche in ragione della prevalente componente di rappresentanza dei lavoratori al suo interno. Ciò che forse lo distingue rispetto al simile Comitato «paritetico» disciplinato nell'ordinamento spagnolo (Comité de Seguridad y Salud), sul quale tuttora diverse sono le perplessità avanzate da parte della dottrina ${ }^{60}$.

Sulla carta, quantomeno, non mancano le condizioni perché il Comité d'hygiène, de sécurité et des conditions de travail operi in senso propositivo e di ricerca delle migliori soluzioni prevenzionistiche ${ }^{61}$.

$\mathrm{Va}$, infine, esclusa una sua assimilazione rispetto agli organismi paritetici presenti a livello territoriale nell'ordinamento italiano e specificamente regolati dall'art. 51, d.lgs. n. 81/2008, che semmai costituiscono un termine di paragone con altri istituti propri del contesto francese, composti da rappresentanti delle organizzazioni dei datori di lavoro e dei lavoratori, con il compito di promuovere la formazione in materia e di contribuire al miglioramento delle condizioni di salute e sicurezza, ossia gli Organismes professionnels de santé, de sécurité et des conditions de travail e le Commissions de santé et de sécurité (art. L 4643, Code du travail).

\section{Il Comité de Seguridad y Salud}

Tra gli istituti inseriti nella struttura organizzativa dell'impresa e specializzati nella protezione della salute e sicurezza sul lavoro, un ruolo affine a quello del Comité d'hygiène, de sécurité et des conditions de travail è svolto dal Comité de Seguridad y Salud proprio dell'ordinamento spagnolo e regolato agli artt. 38 e 39 della Ley 31/1995, de 8 de noviembre, de Prevención de Riesgos Laborales, ove è contenuta una disciplina alquanto concisa, spesso integrata dalla contrattazione collettiva.

60 Si veda il paragrafo 4.

61 Cfr. Les CHSCT, entre dispositifs et pratiques, Anact, Janvier, 2012, cit., p. 101 ss., ove si chiarisce che spesso nella realtà pratica il funzionamento effettivo e l'azione del CHSCT sono difficili e complessi, dipendendo da diversi fattori tra i quali il tempo necessario per l'espletamento delle funzioni da parte dei componenti. 
La Ley 31/1995 prevede la creazione necessaria di un Comité de Seguridad y Salud in tutte le imprese o stabilimenti con almeno cinquanta lavoratori. In mancanza di una specifica previsione legislativa è, invece, controversa la costituzione di un Comité «conjunto» nell'ipotesi in cui l'impresa abbia più stabilimenti nei quali individualmente non si raggiunga il numero di lavoratori sufficienti al fine di istituire un Comité de Seguridad y Salud ${ }^{62}$.

Ove l'impresa abbia più stabilimenti dotati di un Comité, la Ley accorda la possibilità di istituire un Comité Intercentros, tramite un «acuerdo» con i lavoratori, ossia nella prassi, tramite un contratto collettivo non necessariamente statutario, dal contenuto concreto e preciso (art. $38.3 \mathrm{LPRL}$ ); le funzioni del Comité Intercentros sono individuate nello stesso contratto. Si tratta, in tal caso, di una misura eventuale ed aggiuntiva, normalmente oggetto della contrattazione collettiva empresarial.

V'è, altresì, da sottolineare che questo ulteriore istituto non è da confondere, seppur ad esso somigliante, con il Comité Intercentros regolato dall'art. 63.3 dell'Estatuto de los trabajadores, costituito e disciplinato, in tal caso esplicitamente, «sólo por convenio colectivo»; è ivi prefigurata la facoltà di un Comité Intercentros rispetto al Comité de empresa, «organo rappresentativo e collegiale», a tutela degli interessi dei lavoratori (art. 63.1 ET).

Quanto al Comité de Seguridad y Salud ci si riferisce ad un «órgano de participación» realmente paritetico, rectius, «paritario e collegiale», volto alla consultazione regolare e periodica sulle iniziative del datore di lavoro in materia di prevenzione dei rischi (art. 38.1 LPRL $)^{63}$. La sua composizione comprende, infatti, un numero uguale di delegados de prevención e di rappresentanti datoriali ${ }^{64}$. In

62 Al riguardo cfr. A. Cámara Botía, F.A. González Díaz, Derechos de participación y representación en materia de seguridad y salud laborales: un estudio sobre su aplicación, in Revista del Ministerio de Trabajo y Asuntos Sociales, 2004, n. 53, p. 230. In giurisprudenza v., in particolare, STS de 3 de diciembre de 1997; TSJ de Cataluña de 6 de junio de 2000.

63 Si veda, altresì, l'art. $34 L P R L$, ove è disposto che «los trabajadores tienen derecho a participar en la empresa en las cuestiones relacionadas con la prevención de riesgos en el trabajo».

${ }^{64}$ In proposito v. F. VALDÉs DAL-RÉ, La participación de los trabajadores en la Ley de prevención de riesgos laborales, in Derecho y Salud, 1996, vol. IV, p. 71, 
particolare, i delegados de prevención sono definiti nella Ley come «i rappresentanti dei lavoratori con funzioni specifiche in materia», designati da e tra $\mathrm{i}$ «representantes del personal» ${ }^{65}$.

Similmente a quanto predisposto per il Comité d'hygiène, de sécurité et des conditions de travail, la Ley disciplina la partecipazione nell'ambito delle riunioni del Comité de Seguridad y Salud «con voce ma senza voto» di altri soggetti, tra i quali i delegati sindacali e i responsabili tecnici della prevenzione che non facciano parte del Comité, nonché i lavoratori con una speciale qualifica rispetto alle questioni da trattare e i tecnici esterni all'azienda, se richiesti da parte di alcuni dei rappresentanti presenti nel Comité66 ${ }^{6}$. Le riunioni sono previste trimestralmente (art. 38.3 LPRL).

La regolamentazione dell'istituto è contenuta nel capitolo $\mathrm{V}$ dedicato alla «consulta y participación de los trabajadores» ${ }^{67}$. Per la verità, come fin da subito chiarito in dottrina, il capitolo V non esaurisce la disciplina di tutte le modalità partecipative dei lavoratori in materia, occupandosi solamente dei diritti di consultazione e par-

secondo cui «el legislador ha optado por dotar al Comitè de la manifestación más estricta y externa del paritarismo».

65 L'incertezza della disciplina legale ha, in verità, dato luogo ad un contrastato dibattito in dottrina e in giurisprudenza con particolare riferimento alla possibilità che i delegados de prevención siano designati da e tra la representación unitaria (Comités de Empresa e delegados de personal) e la representación sindical (delegados sindicales). La tesi seguita dalla giurisprudenza (STS 19 de octubre 1998) e dalla prevalente dottrina pare quella che privilegia la scelta all'interno della representación unitaria. Sul tema cfr. M.N. Moreno VIDA, Los derechos de participación y representación de los trabajadores en materia de prevención de riesgos. El Delegado de prevención, cit., p. 641; nonché M.T. Igartua Miró, Sistema de prevención de riesgos laborales, Madrid, Tecnos, 2015, p. 332 ss.

66 La partecipazione dei delegados sindicales alle riunioni del Comité de Seguridad y Salud «con voz pero sin voto» è, inoltre, prevista nell'art. 10.3 della Ley Orgánica de Libertad Sindical (11/1985).

67 In merito alla partecipazione dei lavoratori e loro rappresentanze si veda, in particolare, E. GonzÁlez-Posada Martínez, La representación de los trabajadores en materia de salud laboral, in F. Valdés Dal-Ré, M.L. Molero Marañón (dir.), La representación de los trabajadores en las nuevas organizaciones de empresa, Madrid, MTIN \& Fundación Largo Caballero, 2010, p. 183 ss.; E. Garrido Pérez, La participación de los trabajadores en materia de prevención de riesgos laborales en la Ley 31/1995, in Relaciones Laborales, 1997, n. 2, p. 397 ss. 
tecipazione $^{68}$; si deve, in effetti, richiamare per completezza l'art. 18 LPRL, esplicitamente dedicato a «información, consulta y participación de los trabajadores», nonché l'art. 2 LPRL, che rimarca l'obiettivo della Ley di stabilire i principi generali per la protezione dei lavoratori e la loro «informazione, consultazione e partecipazione equilibrata», che sono parte del diritto ad una «protezione efficace» (art. 14.1 LPRL). I prestatori di lavoro, ai sensi del citato art. 18.2, hanno, in particolare, il diritto di effettuare proposte al datore di lavoro e agli organi di partecipazione e di rappresentanza di cui al capitolo V, per migliorare i livelli di sicurezza e salute nell'impresa.

Il Comité de Seguridad y Salud è privo della capacità di negoziare, come si addice del resto ai comitati già presi in esame e presenti in altri contesti nazionali, e ha funzioni prevalentemente tecniche e di consulenza, senza che assuma rilevanza l'appartenenza sindacale dei delegati ${ }^{69}$.

Le competenze affidate al Comitato sono essenzialmente quelle di partecipare alla «elaborazione, messa in pratica e valutazione dei piani e programmi di prevenzione dei rischi nell'impresa»e di «promuovere iniziative sui metodi e procedimenti per l'effettiva prevenzione dei rischi» attraverso la proposta di miglioramento delle condizioni e di correzione delle mancanze esistenti (art. 39.1 LPRL). Il Comité assume, allora, il ruolo di discutere in ordine alla realizzazione delle attività preventive, dei progetti di organizzazione del lavoro e di introduzione di nuove tecnologie, in stretta connessione con quanto disposto dall'art. $16 L P R L$, che si riferisce propriamente all'attuazione del «plan de prevención de riesgos laborales», alla «evaluación de los riesgos y planificación de la actividad preventiva». L'intervento dell'organismo partecipativo abbraccia, dunque, la politica preventiva dell'impresa, avendo

68 F. VALDÉs DAL-RÉ, La participación de los trabajadores en la ley de prevención de riesgos laborales, cit., p. 49 ss.

${ }^{69} \mathrm{Si}$ deve, inoltre, precisare che ai sensi dell'art. 35.4 LPRL residua la possibilità che i contratti collettivi stabiliscano altri criteri di designazione dei delegados de prevención. Quanto alle problematiche relative alla costituzione e composizione del Comitè de Seguridad y Salud cfr., in particolare, M.C. Aguilar del Castillo, El Comité de Seguridad y Salud, cit., p. 63 ss. 
ad oggetto, non casualmente, l'obbligazione generale a carico del datore di lavoro ${ }^{70}$.

Nell'esercizio di tali compiti, il Comitato deve conoscere direttamente la situazione relativa alla prevenzione dei rischi, effettuando nell'ambito dello stabilimento le visite che ritiene opportune, ed essere al corrente delle informazioni necessarie per l'adempimento delle proprie funzioni, come anche dei danni alla salute dei lavoratori, così da valutarne le cause e proporre adeguate misure preventive (art. 39.2 LPRL).

Assume, pertanto, importanza l'attività partecipativa condotta dal Comité de Seguridad y Salud, che si caratterizza in funzione del suo ruolo propositivo e collaborativo. A differenza dell'esaminato Comité d'hygiène, de sécurité et des conditions de travail, ove preponderante è la componente della rappresentanza dei lavoratori, il Comité spagnolo, in quanto istituto paritario, si distingue più correttamente come organismo collegiale partecipativo, non propriamente rappresentativo del personale. $\mathrm{Ci}$ si potrebbe chiedere, da questo punto di vista, se la sua composizione indebolisca in parte la possibilità di incidere sulle decisioni manageriali, potendo dipendere il suo efficace funzionamento dalla capacità di pressione esercitata dai rappresentanti dei lavoratori presenti.

Come risulta dalle previsioni legislative, il Comité de Seguridad y Salud è esattamente incaricato di rilasciare opinioni e pareri, così come di formulare suggerimenti diretti a conseguire una effettiva tutela dei lavoratori nell'ambiente di lavoro, potendo svolgere una sorta di «controllo interno» sul processo di adozione delle decisioni datoriali ${ }^{71}$. Un organo «de encuentro» tra le rappresentanze del personale e il datore di lavoro, volendo usare le parole contenute nella $E x$ posición de motivos de la Ley de Prevención de Riesgos Laborales.

L'azione del Comité si esplicita, in sostanza, nel facilitare e garantire un miglioramento delle condizioni di salute ${ }^{72}$.

$70 \mathrm{Si}$ vedano le osservazioni al riguardo di M.C. Aguilar del Castillo, El Comité de Seguridad y Salud como órgano de participación de los trabajadores en la negociación colectiva, cit., p. 113 ss.

71 F. VAldés DaL-Ré, La participación de los trabajadores en la ley de prevención de riesgos laborales, cit., p. 73.

72 M.C. Aguilar del Castillo, El Comité de Seguridad y Salud, cit., p. 304. 
Di non poca importanza è poi, come anticipato, il ruolo assunto dalla fonte negoziale nel regolare ed integrare gli aspetti della informazione, consultazione e partecipazione dei lavoratori. La contrattazione collettiva, prevalentemente a livello d'azienda, contempla, in effetti, clausole migliorative rispetto alle disposizioni normative sia con riguardo, ad esempio, al numero dei Delegados de Prevención presenti all'interno dell'impresa, sia rispetto all'introduzione del $C o$ mité Intercentros, lasciata alla libera discrezionalità del datore di lavoro dalla Ley, nonché alla frequenza con la quale il Comité de Seguridad y Salud si riunisce ${ }^{73}$.

La stessa Estrategia Española de Seguridad y Salud 2015-2020, che rappresenta il quadro generale di riferimento delle politiche di prevenzione dei rischi ed orienta le azioni da intraprendere da parte delle istituzioni competenti nella protezione della salute nei luoghi di lavoro, enfatizza la rilevanza della partecipazione attiva dei lavoratori, che non deve limitarsi alla consultazione ed informazione, bensì comprendere la loro «completa collaborazione con il datore di lavoro», cosicché la prevenzione dei rischi diventi «un'attività comune»; in questa direzione sono incentivate le iniziative datoriali contraddistinte da un processo decisionale comune proprio nell'ambito del Comité de Seguridad y Salud, il cui ruolo di organo partecipativo specializzato è ampiamente valorizzato ${ }^{74}$.

Non si può, tuttavia, trascurare di prendere in esame le conseguenze dell'eventuale omissione datoriale del dovere di interpellare tale organismo, che può condurre ad una irregolarità destinata ad

$73 \mathrm{Si}$ veda, a titolo esemplificativo, il Convenio colectivo del personal de tierra de Iberia, Líneas Aéreas de España, SA, Operadora, S. Unipersonal, del 6 maggio 2014, in BOE 22 maggio 2014, ove il ruolo svolto dal Comité è espressamente definito come «fondamentale» nella promozione della tutela della sicurezza sul lavoro e per tale motivo oggetto di particolare attenzione; il Convenio colectivo de Retevisión I, SAU, del 6 maggio 2014, in BOE 19 maggio 2014.

74 Già la Estrategia Española de Seguridad y Salud 2007-2012 ha sottolineato che per il tramite del Comité sono discusse, in sostanza, le questioni relative alle modalità di organizzazione dell’impresa. In ordine alle «strategie di prevenzione dei rischi» adottate a livello dell'Unione europea e nell'ordinamento spagnolo cfr. le riflessioni di A. Montoya Melgar, Estrategias y planes sobre prevención de riesgos laborales, in Nueva Revista Española de Derecho del Trabajo, 2015, p. 13 ss. 
inficiare l'efficacia della decisione finale ${ }^{75}$. Anche se il descritto meccanismo non è certamente definibile come codecisionale, il Real Decreto Legislativo 5/2000, con il quale è approvato il testo della Ley sobre Infracciones y Sanciones en el Orden Social, ha previsto tra le infrazioni gravi, in materia di prevenzione dei rischi, «la violazione dei diritti di informazione, consultazione e partecipazione dei lavoratori» (art. 12.11).

Occorre, inoltre, sottolineare che il capitolo VI, contenuto nel citato Real Decreto Legislativo 5/2000 e dedicato alle «responsabilità e sanzioni», si riferisce, nell'art. 39, ai «criteri di graduazione delle sanzioni». Precisamente, rispetto alle sanzioni conseguenti a infrazioni nella materia de qua, quali criteri di graduazione saranno considerati tra gli altri «la mancata osservanza delle proposte realizzate dal Comité de seguridad y salud» (art. 39.3, lett. g).

In definitiva, il parere rilasciato dal Comité, regolarmente consultato, non è vincolante, potendo il datore di lavoro adottare la propria decisione senza tenerlo in considerazione. Nella prospettiva sanzionatoria, tuttavia, non acquisire le considerazioni o conclusioni cui l'istituto è pervenuto, costituisce un criterio per graduare le sanzioni relative alle infrazioni tipizzate dal legislatore, che potranno in tal modo essere incrementate.

Pur privo del potere di negoziazione, il suo ruolo potrebbe, in definitiva, divenire determinante, dovendo essere comunque soddisfatta l'esigenza di previa discussione e condivisione delle principali problematiche legate alla protezione della salute in azienda.

5. Gli accordi di codeterminazione nella tutela della sicurezza sul lavoro (Betriebsvereinbarungen)

In merito alle tematiche affrontate non si può, come anticipato, prescindere dal prendere in esame l'ordinamento tedesco, tentando una seppur breve comparazione con l'istituto della Mitbestimmung, l'espressione più esemplare della partecipazione dei lavoratori.

75 F. VAldés Dal-Ré, La participación de los trabajadores en la ley de prevención de riesgos laborales, cit., p. 73. 
Tale «parola magica» ${ }^{76}$ rappresenta essenzialmente la codeterminazione aziendale, la cui disciplina è contenuta nel Betriebsverfassungsgesetz (BetrVG), la legge sulla costituzione aziendale del 1952, modificata sostanzialmente nel 1972 e rinnovata nuovamente nel $2001^{77}$.

Il consiglio aziendale (Betriebsrat), ossia l'organo eletto dalla generalità dei lavoratori e portatore dei loro interessi, esercita i diritti riconosciuti dal BetrVG, tra i quali i diritti di informazione, di consultazione, di codeterminazione e di controllo ${ }^{78}$.

Vige, come noto, a differenza dell'ordinamento italiano, una rappresentanza degli interessi che si fonda sul doppio canale: quello del consiglio d'azienda, portatore delle istanze di tutti i lavoratori impiegati in una determinata impresa, e quello dei sindacati, rappresentanti degli interessi dei propri iscritti. Il Betriebsrat è un organo indipendente rispetto alle organizzazioni sindacali e l'adesione all'associazione sindacale non costituisce un presupposto per la partecipazione al consiglio, anche se molto spesso i suoi componenti sono lavoratori appartenenti ad una delle organizzazioni sindacali ${ }^{79}$.

76 M. Pedrazzoli, La partecipazione dei lavoratori: un tema di natura costituzionale e politica, in C. ZOLI (a cura di), Lavoro e impresa: la partecipazione dei lavoratori e le sue forme nel diritto italiano e comparato, cit., p. 8 ss.; sul tema già ID., Alternative italiane sulla partecipazione nel quadro europeo: la cogestione, in DLRI, 1991, p. 14 ss.

$77 \mathrm{Al}$ riguardo cfr., in particolare, W. DÄUBLER, Diritto sindacale e cogestione nella Germania Federale, ed. it. accresciuta a cura di M. Pedrazzoli, Milano, Franco Angeli, 1981, p. 297 ss.; U. ZaChert, Lezioni di diritto del lavoro tedesco, in Quaderni del Dipartimento di Scienze Giuridiche dell'Università degli Studi di Trento, 1995, p. 65 ss.

78 Il Betriebsrat è eletto ogni quattro anni in tutte le unità produttive con almeno cinque dipendenti a tempo indeterminato, di cui tre eleggibili. Il numero dei suoi componenti varia in relazione ai lavoratori dell'azienda: cfr. $§ 1$ e ss. BetrVG. Per una recente riflessione sui diritti riconosciuti al consiglio aziendale cfr. M. BoRZAGA, Il decentramento "controllato" della contrattazione collettiva nella Repubblica federale tedesca come antidoto alla crisi economica: realtà o mito?, in DLRI, 2015, p. 280 ss.

79 Quanto ai poteri affidati al sindacato in merito alla formazione del consiglio aziendale cfr., in particolare, il $\S 14$, comma 3, BetrVG. In ordine al rapporto tra le organizzazioni sindacali e il consiglio d'azienda v. le considerazioni di W. DäUBler, Trade Union Rights at the Workplace in Germany, in R. BlanPaIN (ed.), Trade Union Rights at the Workplace, Alphen aan den Rijn, Wolters Kluwer, 2012, p. 64 ss. 
In particolare, ai sensi del $\S 80$ del BetrVG, tra i compiti generali del consiglio aziendale, v'è anzitutto l'obbligo di vigilare in ordine al rispetto delle leggi, dei regolamenti, delle disposizioni per la prevenzione degli infortuni, nonché dei contratti collettivi e delle «pattuizioni aziendali» a tutela dei lavoratori ${ }^{80}$, e di richiedere al datore di lavoro la promozione dell'uguaglianza sostanziale tra uomini e donne e della conciliazione tra famiglia e lavoro.

Il § 80, comma 1, n. 9, affida, inoltre, al Betriebsrat il compito di incentivare l'adozione di misure per la tutela della sicurezza e dell'ambiente di lavoro ${ }^{81}$. Per il perseguimento di tali funzioni vige in capo al datore di lavoro l'obbligo di informare tempestivamente ed esaustivamente il consiglio d'azienda, nonché di mettere a sua disposizione i documenti necessari ( $§ 80$, comma 2, BetrVG). I diritti di informazione del consiglio aziendale sono specificati nel $\S 90$ e riguardano i processi e l'organizzazione del lavoro; i diritti di consultazione tempestiva concernono le misure previste ed i loro effetti e consentono al consiglio aziendale di manifestare dubbi e consigli (§ 90, comma 2).

$\mathrm{E}$, in ogni caso, sancito che datore di lavoro e consiglio d'azienda devono collaborare e contribuire al buon andamento dell'azienda (cfr. § 2, comma 1, BetrVG) ${ }^{82}$.

Quanto, esattamente, alla tutela della sicurezza e dell'ambiente lavorativo, ad integrazione delle specifiche disposizioni contenute nella legislazione di riferimento ossia nell'Arbeitsschutzgesetz, la legge sulla costituzione aziendale rimarca il ruolo del $B e-$ triebsrat, collocato «al centro del sistema di sicurezza aziendale», di garantire l'attuazione delle disposizioni prevenzionistiche, ed il suo coinvolgimento nelle ispezioni sugli infortuni sul lavoro ( $\$ 89$ $\operatorname{Betr} V G)^{83}$.

80 Cfr. M. BIASI, Il nodo della partecipazione dei lavoratori in Italia. Evoluzioni e prospettive nel confronto con il modello tedesco ed europeo, Milano, Egea, 2013, p. 71 ss.

$81 \mathrm{~V}$. in proposito U. FABer, Vorbemerkungen zu $\S 87$ BetrVG, in W. Kohte, U. FABER, K. FeldHOFF (hrsg.), Gesamtes Arbeitsschutzrecht, cit., 2014, p. 1259 ss.

82 V. le considerazioni di U. ZACHERT, Lezioni di diritto del lavoro tedesco, cit., p. 71, che sottolinea come il tipo di relazioni industriali proprio del sistema tedesco è «basato su una forte cooperazione tra gli attori aziendali».

83 M. ConTI, La partecipazione dei lavoratori. La cornice europea e l'esperienza comparata, cit., p. 135 . Per un commento al $\S 89$ v. T. KANIA, $\$ 89$ Arbeits- 
Come precisato in dottrina, la materia della salute e sicurezza nei luoghi di lavoro è tradizionalmente al centro dell'organizzazione aziendale; ciò si evince dalle disposizioni che il BetrVG vi dedica, assurgendo in tal modo a strumento centrale per l'applicazione del diritto della sicurezza del lavoro ${ }^{84}$.

A destare maggiore interesse, trattandosi di peculiarità che contraddistinguono l'ordinamento in esame, sono, senza dubbio, le regole relative al diritto di codeterminazione contemplate al $\S 87 \mathrm{Be}$ trVG, nonché quelle riferentisi alla proposta di misure aggiuntive da parte del consiglio di cui al $\S 88$ BetrVG. Tali previsioni assumono importanza proprio dal punto di vista della tutela della salute dei lavoratori e costituiscono un unicum difficilmente rinvenibile in altri ordinamenti nazionali.

Lo stimolante confronto con queste incisive forme di coinvolgimento dei prestatori di lavoro si rende necessario, al fine del presente studio, in quanto solleva rilevanti questioni in termini di volontarietà e obbligo nella materia, contribuendo ad arricchire le riflessioni che precedono.

Va sottolineato che le questioni sulle quali il consiglio d'azienda esercita i propri diritti e prerogative sono suddivise in «sociali» (soziale Angelegenheiten), «del personale» (personelle Angelegenheiten) ed «economiche» (wirtschaftliche Angelegenheiten). Ma, come chiarito, il maggior coinvolgimento dell'organo di rappresentanza si rinviene nelle questioni sociali, che si riferiscono a varie materie rispetto alle quali i diritti di codeterminazione sono molto intensi ${ }^{85}$.

Ai sensi del $\S 87$, comma 1, n. 7, BetrVG tra le questioni sociali tassativamente elencate emerge la disciplina della prevenzione degli infortuni sul lavoro, delle malattie professionali e della tutela della salute, nel quadro delle disposizioni legislative in materia. Si tratta, in sostanza, dell'attività di negoziazione tra datore di lavoro e consiglio d'azienda e della conclusione di accordi aziendali

und betrieblicher Umweltschutz, in R. Müller-Glöge, U. Preis (hrsg.), Erfurter Kommentar zum Arbeitsrecht, München, Verlag C.H. Beck, 2010, p. 1180 ss.

84 U. FABER, Vorbemerkungen zu $\S 87$ BetrVG, cit., p. 1257 ss.

85 M. Pedrazzoli, La partecipazione dei lavoratori: un tema di natura costituzionale e politica, cit., p. 9. In proposito v. M. WeIss, L'effettività del diritto del lavoro: alcune riflessioni sull'esperienza tedesca, in RIDL, 2006, I, p. 158. 
(Betriebsvereinbarungen), nell'ambito del diritto di codeterminazione, altrimenti definito in dottrina «diritto alla coregolamentazione» (Mitregelungsrecht $)^{86}$.

Dunque, accanto a organizzazioni sindacali dei lavoratori e dei datori di lavoro, anche consiglio aziendale e datore di lavoro hanno potere di regolamentazione nei limiti di quanto stabilito nel BetrVG. Anzi, gli esiti della Mitbestimmung hanno assunto notevole rilevanza anche rispetto alla contrattazione collettiva decentrata ${ }^{87}$.

Occorre, a questo punto, riflettere sul contenuto esatto, sulla natura, nonché sull'efficacia di tali accordi.

La codeterminazione è la forma più forte di partecipazione; infatti, se una misura è oggetto del diritto di codeterminazione, può essere adottata solo previo consenso del consiglio aziendale, non unilateralmente, e ove non venga raggiunto un accordo in merito, la questione è decisa in sede di conciliazione. Come espressamente sancito dal § 76 BetrVG, al collegio arbitrale è attribuito il potere di risoluzione delle divergenze di opinioni tra le parti a livello aziendale e di adozione di una decisione vincolante in luogo del mancato accordo $^{88}$. Il Betriebsrat, attraverso l'esercizio del suo diritto di codeterminazione (Mitbestimmungsrecht), può, dunque, influenzare e vincolare il processo decisionale del datore di lavoro, ostacolandone il proseguimento con la devoluzione della controversia nelle mani di un terzo neutrale.

Più precisamente il datore di lavoro, in quanto destinatario dell'obbligo di protezione della salute dei lavoratori, è tenuto a presentare al consiglio aziendale le disposizioni da adottare in materia, al fine di ottenere il suo consenso ${ }^{89}$. In caso contrario, infatti, il con-

86 R. RICHARDI, $\S 87$ Mitbestimmungsrechte, in ID. (hrsg.), Betriebsverfassungsgesetz mit Wahlordnung, München, Verlag C.H. Beck, 2016, p. 1416.

87 M. BorZAGA, Il decentramento "controllato" della contrattazione collettiva nella Repubblica federale tedesca come antidoto alla crisi economica: realtà o mito?, cit., p. 297.

88 Quanto alla costituzione del collegio arbitrale interno il § $76 \mathrm{BetrVG}$ precisa che i suoi componenti sono nominati, in ugual numero, dal datore di lavoro e dal consiglio aziendale, mentre il presidente «imparziale» è scelto d'accordo tra le parti o dal Tribunale del lavoro, ove non si raggiunga un accordo comune.

89 D. Nitsche, $\S 87$ BetrVG Mitbestimmungsrechte, in W. KoHte, U. Faber, K. FeldHOFf (hrsg.), Gesamtes Arbeitsschutzrecht, cit., p. 1305 ss. 
siglio può esercitare una sorta di "veto" (Unterlassungsanspruch), cui consegue l'eliminazione delle disposizioni medesime assunte senza il suo parere ${ }^{90}$.

Ma anche il Betriebsrat può prendere l'iniziativa e conseguire coattivamente l'adozione di misure aziendali soddisfacenti e rispettose delle esigenze di entrambe le parti. Il diritto di iniziativa (Initiativrecht), che si affianca al diritto di dare il proprio consenso ( $\mathrm{Zu}$ stimmungsrecht), non si desume direttamente dal $\S 87$ BetrVG, bensì dal «significato e dalla finalità del diritto di codeterminazione» ${ }^{91}$; la Mitbestimmung include, quindi, un diritto di iniziativa del consiglio d'azienda. Questo diritto, definito «illimitato», deve però manifestarsi in una proposta concreta e non in una mera esortazione al datore di lavoro ad attuare e specificare il quadro normativo di riferimento ${ }^{92}$.

Occorre ricordare, a questo punto, la previsione contenuta nel $\S 91$ BetrVG, che attribuisce al consiglio un preciso potere d'intervento a fronte di modifiche del processo o ambiente lavorativo lesive per i lavoratori ed in evidente contrasto con un'organizzazione del lavoro rispettosa della persona. In tal caso al consiglio d'azienda è attribuito il diritto di richiedere l'adozione di misure idonee ad ovviare, ridurre o compensare gli effetti che ne conseguono. Tuttavia, è stata in un certo senso sminuita la portata della norma rispetto al contenuto del $\S 87$. Si tratta, in effetti, di un diritto di codeterminazione c.d. «correttivo», esercitabile cioè non in via preventiva,

90 Cfr. BAG 16.06.1998, in NZA 1999, p. 49, ove è precisato che il consiglio aziendale, qualora il datore di lavoro abbia violato il diritto di codeterminazione ed assunto e comunicato unilateralmente una decisione, può chiederne la sua eliminazione.

91 R. Richardi, $\& 87$ Mitbestimmungsrechte, in ID. (hrsg.), Betriebsverfassungsgesetz mit Wahlordnung, cit., p. 1304 ss.

92 T. KLEBE, $\$ 87$ Mitbestimmungsrechte, in W. Däubler, M. KitTNER, T. KLEBE, P. Wedde (hrsg.), Betriebsverfassungsgesetz. Kommentar für die Praxis mit Wahlordnung und EBR-Gesetz, Frankfurt am Main, Bund Verlag, 2014, p. 1687; D. NiTsche, $\S 87$ BetrVG Mitbestimmungsrechte, cit., p. 1306, che ha chiarito come entrambe le parti siano tenute a concretizzare le proprie proposte. In merito al diritto d'iniziativa del consiglio aziendale cfr. BAG 31.08.1982, in $D B, 1983$, p. 453; più di recente v. BAG 15.01.2002, in Arbeitsrechtliche Praxis, n. 12, § $87 \mathrm{Be}$ $\operatorname{trVG}$; nonché BAG 11.01.2011, in NZA, 2011, p. 651, che ha ribadito la necessità di proposte concrete da parte del consiglio aziendale in particolare con riferimento al $\S 12$ ArbSchG. In proposito v. T. KANIA, $\S 87$ Mitbestimmungsrechte, in R. MüLLER-GlögE, U. PreIS (hrsg.), Erfurter Kommentar zum Arbeitsrecht, cit., p. 1152 ss. 
bensì solo a fronte di intervenute scelte datoriali volte ad incidere sull'organizzazione del lavoro con pregiudizio per i prestatori; dunque, una volta che si siano verificate le condizioni contemplate nel BetrVG $G^{93}$.

Come si ricava dal $\S 77$, gli accordi aziendali devono essere decisi congiuntamente fra consiglio e datore di lavoro, pur rimanendo quest'ultimo il destinatario degli obblighi ed il responsabile per l'attuazione delle norme a tutela della salute dei prestatori di lavoro.

La menzionata previsione di cui al $\S 87$, comma 1, n. 7, Be$\operatorname{tr} V G$ persegue il fine di garantire, mediante la partecipazione del Betriebsrat, una tutela della salute nell'ambiente di lavoro che sia efficace e ritagliata sulla realtà aziendale; le previsioni legislative in materia possono, in tal modo, essere concretizzate a livello della singola azienda, in ossequio all'art. 2 della Costituzione (Grundgesetz) ${ }^{94}$.

Assume particolare rilievo da questo punto di vista il $\S 3$ $A r b S c h G$, che impone al datore di lavoro di adottare le «misure necessarie» (erforderlichen Maßnahmen des Arbeitsschutzes) nel rispetto delle «circostanze» che influiscono sulla salute e sicurezza dei prestatori di lavoro, includendo il miglioramento delle misure di prevenzione dei rischi, a garanzia dell'integrità fisica e psichica dei lavoratori. Non è forse azzardato accostare il $\S 3 \mathrm{ArbSch} G$ alla previsione dell'art. 2087 c.c., considerato da sempre norma aperta, in ragione appunto dell'obbligo imposto all'imprenditore di adottare le «misure necessarie» secondo i noti parametri mutevoli nel tempo della particolarità del lavoro, oltreché dell'esperienza e della tecnica. In linea con il $\S 3 \mathrm{ArbSch} G$ è riconosciuto al consiglio aziendale un ampio diritto di codeterminazione ex $\S 87$, comma 1 , n. 7, concernente le condizioni organizzative e tecniche del lavoro.

V'è, tuttavia, da rimarcare che tale diritto è esercitabile «nel quadro delle disposizioni legislative in materia», presupponendo una specificazione, non un innalzamento del livello di tutela impo-

93 W. DäUBler, Diritto sindacale e cogestione nella Germania Federale, cit., p. 374.

94 T. Klebe, $\S 87$ Mitbestimmungsrechte, in W. DäUbler, M. Kittner, T. KleBE, P. Wedde (hrsg.), Betriebsverfassungsgesetz. Kommentar für die Praxis mit Wahlordnung und EBR-Gesetz, cit., p. 1761 ss. 
sto per via legislativa ${ }^{95}$. La possibilità di introdurre regole supplementari ed innovative è, infatti, individuata nel $\S 88$, dal contenuto alquanto differente, come si cercherà di chiarire.

Inoltre, secondo l'incipit del $\S 87$, comma 1, il consiglio aziendale, come garante dei singoli lavoratori, esercita il diritto di codeterminazione «qualora non esista alcuna disposizione di legge o di contratto collettivo». Tale formula è stata interpretata nel senso che la Mitbestimmung si basa sull'esistenza di disposizioni in materia di tutela della sicurezza che necessitano appunto di essere specificate, in quanto non esaustive ${ }^{96}$. Datore di lavoro e consiglio aziendale possono, in sostanza, adottare previsioni integrative all'interno dello spazio d'intervento lasciato dalla legge stessa.

L'esercizio del diritto di codeterminazione (Mitbestimmungsrecht) esige, dunque, che vi siano regole legislative da integrare a livello d'azienda. L'ambito di applicazione comprende esattamente le leggi e le Unfallverhütungsvorschriften, che, come già ricordato, costituiscono misure vincolanti per il datore di lavoro, al fine della prevenzione degli infortuni ( $§ 14$ e 15 SGB VII), adottate dalle Associazioni categoriali di assicurazione contro gli infortuni sul lavoro ${ }^{97}$. Deve in ogni caso trattarsi di norme di riferimento che necessitano di essere in un certo senso completate e perfezionate.

Si può menzionare, a mo' di esempio, il rinvio all'esercizio del diritto di codeterminazione relativamente alla nomina e revoca dei medici aziendali e specialisti per la sicurezza ed alla modifica e ampliamento dei loro compiti, contenuto nel $\S 9$, comma 3, dell'Arbeitssicherheitsgesetz (Asig), la già menzionata legge relativa ad alcuni aspetti della tutela della sicurezza del lavoro del 1973, in seguito modificata ${ }^{98}$.

95 T. Kania, $\& 87$ Mitbestimmungsrechte, in R. Müller-Glöge, U. Preis (hrsg.), Erfurter Kommentar zum Arbeitsrecht, cit., p. 1163.

96 BAG 8.06.2004, in Arbeitsrechtliche Praxis, n. 13, § 87 BetrVG, che ha precisato come le disposizioni legislative possono indicare un obiettivo di tutela da raggiungere, richiedendo l'intervento necessario del datore di lavoro e del consiglio d'azienda mediante una regolamentazione aziendale congiunta.

${ }_{97}$ Per ulteriori riferimenti cfr. il capitolo I, paragrafo 8.1.

98 Occorre, tuttavia, precisare che sempre ai sensi del § 9, comma 3, dell'Arbeitssicherheitsgesetz, il consiglio aziendale deve, invece, essere sentito «prima dell'assegnazione o revoca di un incarico ad un medico libero professionista e ad un 
L'ambito di applicazione del Mitbestimmungsrecht è, poi, stato esteso con l'entrata in vigore dell'Arbeitsschutzgesetz, le cui disposizioni lasciano discreti margini di discrezionalità. Ciò si ricava dalla

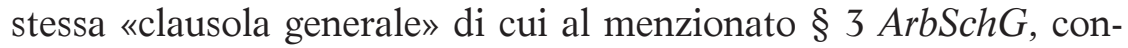
cernente gli obblighi principali del datore di lavoro ${ }^{99}$.

Assumono importanza, da questo punto di vista, il $\S 5 \mathrm{ArbSch} G$, in merito all'obbligo di valutazione dei rischi, nonché il § 12 $\operatorname{ArbSch} G$, relativo all'obbligo di informazione dei lavoratori ${ }^{100}$. La giurisprudenza si è di frequente soffermata proprio sull'obbligo di valutazione dei rischi a carico del datore di lavoro, in ordine al quale la disciplina legislativa, non contenendo previsioni di dettaglio sulle modalità di esecuzione, affida ampi spazi di intervento alla codeterminazione tra consiglio aziendale e datore di lavoro: la principale misura di prevenzione è, quindi, rapportata al singolo contesto della struttura aziendale ${ }^{101}$.

Le ragioni sottese a questa tecnica di regolazione definita flessibile (flexible Regelungstechnik), che si avvale della codeterminazione, sono quelle di consentire una valutazione delle condizioni azien-

addetto alla sicurezza libero professionista»; cfr. per un commento R. AufHAUSER, $\S 9$ Zusammenarbeit mit dem Betriebsrat, in R. AufHauser, H. BrunhöBer, P. IGL, Arbeitssicherheitsgesetz. Handkommentar, Baden-Baden, Nomos, 2010, p. 109 ss.

99 Sul punto BAG 18.03.2014, in NZA, 2014, p. 855, che ha stabilito che il $\S 3 \operatorname{ArbSch} G$ contempla un quadro normativo di riferimento per lo sviluppo di un'organizzazione conforme alle esigenze aziendali ed in particolare alle dimensioni ed al tipo di rischi presenti. In dottrina v. D. NiTsche, $\S 87$ BetrVG Mitbestimmungsrechte, cit., p. 1280, secondo il quale il $33 \mathrm{ArbSchG}$ prevede un «obbligo di adempimento preventivo e generale del datore di lavoro di adottare, programmare ed attuare le misure necessarie per la tutela della sicurezza», svolgendo la funzione di «clausola generale»; nonché A. Blume, U. FABER, $\S 3$ ArbSchG Grundpflichten des Arbeitgebers, in W. Kohte, U. Faber, K. Feldhoff (hrsg.), Gesamtes Arbeitsschutzrecht, cit., p. 103 ss.

100 Quanto al diritto di codeterminazione del consiglio aziendale in merito all'obbligo del datore di lavoro di informazione dei lavoratori ai sensi del $\S 12$

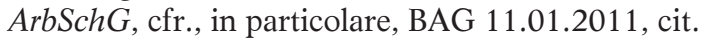

101 Tale principio è rimarcato, ad esempio, da BAG 8.06.2004, cit., nonché da BAG 12.08.2008, in Arbeitsrechtliche Praxis, n. 29, § 618 BGB e BAG 18.08.2009, in NZA, 2009, p. 1434. Si vedano al riguardo le considerazioni di W. Kонте, Die Spontaneität des Südens und die Beständigkeit des Nordens. Impressionen zum kollektivvertraglichen Gesundheitsschutz, in R. ERD, R. FABIAN, E. Kocher, E. ScHMIDT (hrsg.), Passion Arbeitsrecht. Erfahrungen einer unruhigen Generation. Liber amicorum Thomas Blanke, Baden-Baden, Nomos, 2009, p. 176. 
dali specifiche ${ }^{102}$. Il forte coinvolgimento degli stessi destinatari della tutela mira, inoltre, ad un corretto equilibrio fra la libertà di iniziativa economica del datore di lavoro e i diritti fondamentali dei lavoratori riconosciuti all'art. 2 del Grundgesetz.

In definitiva, a fronte di un quadro normativo che si presenta generico, subentra il diritto di attuazione concreta da parte del $B e$ triebsrat, il cui ruolo attivo di garante dei lavoratori pare sempre più rafforzato ${ }^{103}$.

Ciò che contraddistingue la Mitbestimmung e, come anticipato, la separa nettamente da altre forme di partecipazione debole contemplate anche nel nostro ordinamento, è la necessità di raggiungere un accordo tra le parti, in mancanza del quale la decisione viene presa in sede di conciliazione, in sostituzione dell'accordo di codeterminazione.

I limiti cui è sottoposto il diritto di codeterminazione sono esplicitati nel § 77, comma 3: gli istituti retributivi e le condizioni di lavoro regolate dalla contrattazione collettiva non possono, infatti, essere oggetto di accordi di codeterminazione, a meno che la fonte collettiva non preveda espressamente tale intervento integrativo. Il fine è, in sostanza, quello di evitare «situazioni di concorrenza» tra il potere negoziale del Betriebsrat e quello tipico della contrattazione collettiva ${ }^{104}$.

102 A. Blume, U. FABER, $\S 5$ ArbSchG Beurteilung der Arbeitsbedingungen, in W. Kohte, U. Faber, K. Feldhoff (hrsg.), Gesamtes Arbeitsschutzrecht, cit., p. 205 ss. Anche le linee guida della strategia nazionale per la sicurezza sul lavoro (Gemeinsame Deutsche Arbeitsschutzstrategie) del 2008, successivamente modificate nel 2011, sottolineano la necessità che la valutazione dei rischi avvenga con la partecipazione dei lavoratori stessi e si avvalga della loro esperienza e conoscenza.

103 V. le considerazioni al riguardo di M. KitTner, R. Pieper, Sicherheit und Gesundheitsschutz als Handlungsfeld des Betriebsrats, in Arbeitsrecht im Betrieb, 2005, p. 525 ss., ove si sottolinea, tra l'altro, l'importanza dell'informazione e del coinvolgimento dei lavoratori stessi ai sensi dei §§ 15 e 16 ArbSchG.

104 A. Alaimo, Germania, in B. Caruso, M. D’Antona, S. Sciarra (a cura di), Il diritto del lavoro nell'Unione europea. Francia, Germania, Gran Bretagna, Spagna, vol. I, cap. III, cit., p. 393. Quanto al rapporto tra accordo di codeterminazione e contratto collettivo cfr., in particolare, P. BERG, $\S 77$ Durchführung gemeinsamer Beschlüsse, Betriebsvereinbarungen, in W. Däubler, M. KITTNER, T. KLebE, P. Wedde (hrsg.), Betriebsverfassungsgesetz. Kommentar für die Praxis mit Wahlordnung und EBR-Gesetz, cit., p. 1465. 
Dunque, le disposizioni dei contratti collettivi prevalgono sugli accordi aziendali, che possono essere conclusi solo su materie non abitualmente negoziate nei contratti collettivi oppure qualora gli stessi prevedano espressamente tali forme di intervento attraverso le c.d. clausole di apertura contrattuale (Öffnungsklausel) ${ }^{105}$. È, infatti, possibile ed usuale che un contratto collettivo faccia espressamente riferimento al diritto di codeterminazione, attribuendo la possibilità di concludere accordi ex $\S 87$ BetrVG.

Pur in mancanza di un'esatta definizione degli accordi di codeterminazione, il BetrVG si premura di stabilirne la natura ed efficacia. Ai sensi del menzionato $\S 77$, comma 4 , il cui contenuto è tuttora oggetto di discussione in dottrina, è previsto che le Betriebsvereinbarungen sono vincolanti ed hanno efficacia diretta, al pari dei contratti collettivi.

Più esattamente, l'accordo di codeterminazione è considerato un «contratto di natura privata» (privatrechtlicher Normenvertrag), la cui efficacia normativa è paragonabile a quella dei contratti collettivi ${ }^{106}$. Già da tempo è riscontrabile tra gli interpreti l'inclinazione ad assimilare i due istituti, nonché ad applicare i principi sottesi alla contrattazione collettiva anche agli accordi di codeterminazione. Del resto, la stessa impostazione pare presente nella legge sulla costituzione aziendale ove il $\S 77$, commi 4 e 6 , ricorda il contenuto del $\S 4$ della legge sul contratto collettivo del 1949 (Tarifvertragsgesetz). Ai sensi del $\S 4$ del Tarifvertragsgesetz, infatti, le norme del contratto collettivo hanno «efficacia diretta e vincolante» ${ }^{107}$.

Quanto all'ambito di applicazione, l'accordo di codeterminazione vincola tutti i lavoratori dell'azienda, anche non iscritti ai sindacati ${ }^{108}$;

105 T. KanIA, $\$ 87$ Mitbestimmungsrechte, in R. MÜLler-GlÖge, U. PreIS (hrsg.), Erfurter Kommentar zum Arbeitsrecht, cit., p. 1153 ss.

106 In tal senso v. R. RichaRdi, $\$ 77$ Durchführung gemeinsamer Beschlüsse, Betriebsvereinbarungen, in ID. (hrsg.), Betriebsverfassungsgesetz mit Wahlordnung, cit., p. 1095 ss., il quale si interroga sul significato del termine e ripercorre storicamente i riferimenti normativi agli accordi di codeterminazione, rimarcando il presupposto dell'incontro delle volontà delle parti quale requisito fondamentale.

107 È, tuttavia, da precisare che il BetrVG non fa esplicito riferimento al carattere normativo dell'efficacia delle Betriebsvereinbarungen, in tal caso discostandosi dal Tarifvertragsgesetz, che in diverse occasioni rimarca la caratteristica del contratto collettivo quale fonte di produzione di norme giuridiche (Rechtsnormen).

108 T. Kania, $\S 77$ BetrVG, in R. Müller-Glöge, U. Preis (hrsg.), Erfurter Kommentar zum Arbeitsrecht, cit., p. 1105. 
in ciò si avverte la differenza rispetto all'efficacia soggettiva propria dei contratti collettivi, tuttora efficaci solo nei confronti degli iscritti alle organizzazioni stipulanti ${ }^{109}$. In linea generale, infatti, come si ricava dai $\S \S 3$, comma 1, e 4, comma 1, del Tarifvertragsgesetz, vale il criterio dell'associazione al sindacato stipulante (Tarifgebundenheit) ${ }^{110}$.

La Betriebsvereinbarung, pur entro i limiti della sua competenza e nel rispetto della prevalenza conferita alla contrattazione collettiva, si configura, dunque, come uno strumento alquanto rilevante, specialmente in ordine alla tutela prevenzionistica dei lavoratori, posto che conferisce al consiglio d'azienda la possibilità di gestire collettivamente e in modo dinamico le condizioni di lavoro e di "influenzare" l'organizzazione aziendale a tutela e nell'interesse dei creditori di sicurezza ${ }^{111}$. Uno strumento affine e insieme diverso rispetto al contratto collettivo, che, accanto ad esso, costituisce l'altra forma di regolamentazione a livello d'azienda ${ }^{112}$.

109 Cfr. M. Lowisch, V. Rieble, Tarifvertragsgesetz. Kommentar, München, Vahlen, 2012, p. 29 ss.; ai sensi del $§ 5$ del Tarifvertragsgesetz l'efficacia generale dei contratti collettivi può, tuttavia, essere concessa con decreto ministeriale attraverso una procedura ivi indicata. Per una recente riflessione in merito al rilievo della contrattazione collettiva ed alle possibilità di rappresentare gli interessi dei lavoratori al di fuori di tale sistema cfr. W. DäUBLER, La rappresentanza degli interessi dei lavoratori al di là della contrattazione collettiva, in LD, 2015, n. 1, p. 93 ss.

110 In merito all'ambito di applicazione del contratto collettivo, la questione è in realtà, più complessa, dovendosi distinguere, anche secondo le indicazioni del BAG, tra le norme che regolano il rapporto dei singoli lavoratori, e le Betriebsnormen, relative al funzionamento e all'organizzazione dell'impresa (betriebliche und betriebsverfassungsrechtliche Fragen); queste ultime, ai sensi del § 3, comma 2, Tarifvertragsgesetz, si applicano all'intera azienda, il cui datore di lavoro sia vincolato al contratto collettivo. Si veda in proposito M. Franzen, § 3 Tarifgebundenheit, in R. Müller-Glöge, U. Preis (hrsg.), Erfurter Kommentar zum Arbeitsrecht, cit., p. 2619, secondo cui «deve trattarsi di norme che nella realtà sociale, per ragioni sostanziali e giuridiche, possono trovare un'applicazione solo unitaria». Cfr., inoltre, in giurisprudenza BAG 17.6.1997, in NZA, 1998, p. 213.

111 P. BERG, $\S 77$ Durchführung gemeinsamer Beschlüsse, Betriebsvereinbarungen, in W. Däubler, M. KitTner, T. Klebe, P. Wedde (hrsg.), Betriebsverfassungsgesetz. Kommentar für die Praxis mit Wahlordnung und EBR-Gesetz, cit., p. 1463.

112 R. RichaRDI, § 77 Durchführung gemeinsamer Beschlüsse, Betriebsvereinbarungen, in ID. (hrsg.), Betriebsverfassungsgesetz mit Wahlordnung, cit., p. 1095. Sul tema cfr. M. Fuchs, The Evolving Structure of Collective Bargaining in Europe 1990-2004. National Report Germany, in http://eprints.unifi.it/archive/00001160/. 
5.1. Gli accordi aziendali volontari (freiwillige Betriebsvereinbarungen)

Misure prevenzionistiche aggiuntive (zusätzliche Maßnahmen) possono, invece, essere introdotte mediante veri e propri accordi volontari ai sensi del $\S 88$ BetrVG. Tale disposizione, a completamento del diritto di codeterminazione di cui al $\S 87$, sancisce espressamente che «mediante accordi aziendali possono essere regolate misure integrative per la prevenzione degli infortuni sul lavoro e delle malattie professionali» ${ }^{113}$.

Notevoli sono le differenze tra il contenuto del $\S 87$, comma 1, $\mathrm{n}$. 7, e del $\S 88$. Quest'ultima previsione affida al consiglio d'azienda una «competenza generale» per la disciplina di questioni sociali mediante accordi aziendali con il datore di lavoro, senza un'elencazione tassativa, ma meramente esemplificativa, di materie ${ }^{114}$ : accordi contenenti regole effettivamente ulteriori rispetto agli standard garantiti dalla legge.

Il datore di lavoro, in tal caso, agisce volontariamente e non è vincolato all'adozione di una determinata misura sulla base di un quadro normativo. Queste regole vanno oltre le disposizioni legislative e concernono il miglioramento delle condizioni di lavoro e dei dispositivi di protezione individuale. Il riferimento è, ad esempio, al miglioramento delle condizioni di aria, luce e rumore presenti nell'ambiente di lavoro, all'introduzione di una infermeria aziendale, alla previsione di medici e specialisti della sicurezza in numero maggiore rispetto a quanto stabilito dalla legge ${ }^{115}$.

Le problematiche affrontate negli accordi aziendali possono, in realtà, essere molto varie. Desta particolare interesse, da questo

113 Ai sensi del $\S 88$ è oggetto di accordi aziendali volontari anche l'istituzione di benefici e servizi sociali nell'ambito dell'azienda.

114 R. RichaRd, $\$ 88$ Freiwillige Betriebsvereinbarungen, in ID. (hrsg.), Betriebsverfassungsgesetz mit Wahlordnung, cit., p. 1524 ss.

115 Cfr. Berg, $\S 88$ Freiwillige Betriebsvereinbarungen, in W. DÄUBlER, M. Kittner, T. Klebe, P. Wedde (hrsg.), Betriebsverfassungsgesetz. Kommentar für die Praxis mit Wahlordnung und EBR-Gesetz, cit., p. 1824. Ci si riferisce, inoltre, a misure ulteriori che prevedono il rispetto più rigoroso dei valori Mak (Maximale Arbeitsplatzkonzentration) cioè valori soglia/limite di sostanze chimiche: sul punto v. W. Kohte, $\S 88$ Freiwillige Betriebsvereinbarungen, in F.J. Düwell (hrsg.), Betriebsverfassungsgesetz Handkommentar, Baden-Baden, Nomos, 2014, p. 1110 ss. 
punto di vista, come riportato spesso in dottrina, l'espletamento di c.d. concorsi per la sicurezza sul lavoro (Sicherheitswettbewerbe). Si tratta, in particolare, di concorsi a premi interni aventi lo scopo di motivare i prestatori di lavoro a comportamenti consapevoli e collaborativi in merito alla sicurezza nell'ambiente lavorativo, la cui esecuzione è, appunto, oggetto di accordi volontari ${ }^{116}$.

Di frequente, ricorre nei richiamati accordi aziendali facoltativi la previsione di c.d. «circoli» o «tavoli» per la salute aziendale (Gesundheitszirkel), che hanno assunto un ruolo sempre più importante al fine di elevare la sicurezza sul lavoro ${ }^{117}$. Ci si riferisce esattamente alla predisposizione di gruppi di lavoro, formati normalmente da più lavoratori, dal medico aziendale, da un componente del consiglio d'azienda e da un rappresentante del datore di lavoro, volti a formulare proposte concrete per il miglioramento delle condizioni lavorative e a favorire una maggiore partecipazione dei lavoratori all'interno dell'organizzazione. L'obiettivo è, infatti, quello di creare una gestione integrata della salute mediante la partecipazione di tutti gli attori presenti in azienda.

Si può, allora, ritenere che le misure innovative e migliorative inserite negli accordi aziendali, definiti volontari (freiwillige), mirino ad elevare i livelli garantiti dalla legislazione.

In base al $\S 88$ si fuoriesce dalla Mitbestimmung in senso stretto,

116 Sul tema v. R. RichARDI, $\$ 88$ Freiwillige Betriebsvereinbarungen, in ID. (hrsg.), Betriebsverfassungsgesetz mit Wahlordnung, cit., p. 1525. Anche se l'attuazione di tali concorsi per la sicurezza è rimessa alla mera volontà delle parti ex $\S 88 \mathrm{Be}$ $\operatorname{tr} V G$, v'è però da ricordare che sono oggetto di codeterminazione ai sensi del $\S 87 \mathrm{Be}$ $\operatorname{tr} V G$ le misure dirette a coinvolgere i lavoratori ovvero a promuovere comportamenti consapevoli: in proposito v. BAG 24.03.1981 in DB, 1981, p. 1882, ove si fa in particolare riferimento al $\S 87$, comma 1, n. 1, BetrVG, che prevede il diritto di codeterminazione in merito alle «questioni relative all'organizzazione aziendale ed al comportamento dei lavoratori in azienda». V'è, inoltre, da sottolineare che ai sensi del § 87, comma 1 , n. 12, BetrVG, costituiscono oggetto di codeterminazione anche «i principi concernenti le proposte aziendali», considerate quali sistemi e metodi volti al miglioramento tecnico ed organizzativo del lavoro.

117 Cfr., in proposito, W. Konte, $\S 88$ Freiwillige Betriebsvereinbarungen, in F.J. Düwell (hrsg.), Betriebsverfassungsgesetz Handkommentar, cit., p. 1110 ss.; nonché U. NicKel, C. ReIK, Unternehmen und Betriebsrat zusammen für die Gesundheit. Gemeinsam Verantwortung tragen, in Arbeit und Arbeitsrecht. Sonderausgabe, 2014, p. 39 ss., ove si sottolinea l'importanza della collaborazione tra datore di lavoro e creditori di sicurezza al fine di legare saldamente la protezione della salute alla strategia aziendale. 
ossia dall'obbligatorietà del raggiungimento dell'accordo anche in sede conciliativa, dunque dalla possibilità, esercitabile unilateralmente da parte del consiglio d'azienda, di imposizione di una decisione congiunta ${ }^{118}$. Per questo motivo, nella circostanza qui considerata, la posizione giuridica assunta dal consiglio è sostanzialmente diversa $\mathrm{e}$, in un certo senso, più debole. Il perseguimento di un risultato comune non può, infatti, essere forzato contro la volontà del datore di lavoro.

Vero è che il $§ 88$ garantisce al datore di lavoro ed al Betriebsrat la possibilità di concludere accordi aziendali, che producono a loro volta effetti giuridici (normative Wirkung) ${ }^{119}$. Come chiarito dalla giurisprudenza in più occasioni, il $\S 88$ è, dunque, espressione del potere di creare regole «volontarie» ma dotate di effetti giuridici (Setzung normativer Regelungen ${ }^{120}$.

Si deve, però, precisare che, in virtù del § 77, comma 5, BetrVG, è concessa la facoltà di disdetta nel termine di preavviso di tre mesi, salvo che sia convenuto diversamente ${ }^{121}$. È, pertanto, la stessa legge sulla costituzione aziendale a stabilire la possibilità di revoca unilaterale dei trattamenti aggiuntivi col solo limite del preavviso. Tale regola, che pare valere in realtà per entrambe le fattispecie di accordi, codeterminati e volontari (mitbestimmte und freiwillige), è sottoposta al rispetto del principio di tutela dell'affidamento (Vertrauensschutz) ${ }^{122}$.

118 R. RichaRDI, $\$ 88$ Freiwillige Betriebsvereinbarungen, in ID. (hrsg.), Betriebsverfassungsgesetz mit Wahlordnung, cit., p. 1523 ss. Occorre, tuttavia, precisare che ai sensi del $\S 76$, comma 6, BetrVG, non è esclusa la possibilità di ricorrere in sede di conciliazione ove entrambe le parti lo richiedano: in proposito v. il commento di P. Berg, § 76 Einigungsstelle, in W. Däubler, M. Kittner, T. Klebe, P. Wedde (hrsg.), Betriebsverfassungsgesetz. Kommentar für die Praxis mit Wahlordnung und EBR-Gesetz, cit., p. 1421 ss.

119 W. Kонте, § 88 Freiwillige Betriebsvereinbarungen, in F.J. Düwell (hrsg.), Betriebsverfassungsgesetz Handkommentar, cit., p. 1110 ss.

120 V. BAG 7.11.1989, in Arbeitsrechtliche Praxis, n. 46, § 77 BetrVG 1972; BAG 12.12.2006, in Arbeitsrechtliche Praxis, n. 94, § 77 BetrVG 1972; BAG 5.7.2011, in Arbeitsrechtliche Praxis, n. 139, § 87 BetrVG 1972.

121 Cfr. al riguardo W. DäUBler, Diritto sindacale e cogestione nella Germania Federale, cit., p. 363, che ha in passato rimarcato come, in tale circostanza, «il datore di lavoro dispone, all'occasione, di uno strumento di pressione di non poca importanza», potendo «minacciare un ritiro della sua generosità».

122 BAG 18.09. 2001, in NZA, 2002, p. 1164; nonché BAG 19.02.2008, in NZA, 2008, p. 899; in dottrina v. T. Kania, § 77 BetrVG, in R. Müller-GlöGe, U. PreIs (hrsg.), Erfurter Kommentar zum Arbeitsrecht, cit., p. 1118. Inoltre, come chiarito, la possibilità di recesso libero non deve sfociare in abuso del diritto 
Costituisce, piuttosto, una forma di tutela espressamente riservata solo agli accordi di codeterminazione di cui al $\S 87$, la disciplina relativa alla loro ultrattività (Nachwirkung) prevista al § 77, comma 6, BetrVG, ove è stabilito che alla scadenza di un accordo aziendale le disposizioni ivi contenute, che possono essere anche ottenute in sede di conciliazione, hanno efficacia fino a quando non siano sostituite con un altro accordo ${ }^{123}$.

Infine, anche con riferimento all'adozione di accordi aziendali ex $\S$ 88 , non si è mancato di considerare estensibile il limite, di cui al $\S 77$, comma 3, dell'esistenza di contratti collettivi che prevalgono rispetto ad eventuali accordi ${ }^{124}$. Sicché, un contratto collettivo, dal contenuto vincolante ed esaustivo, non escluderebbe solo il diritto di codeterminazione ex $\S 87$, comma $1, \mathrm{n} .7$, bensì anche la possibilità di adottare misure complementari ex $\S 88$. Tale problematica pare, tuttavia, costituire ancora oggetto di dibattito in dottrina, nonché in giurisprudenza ${ }^{125}$.

\section{Brevi riflessioni conclusive}

L'attenzione rivolta ad altri ordinamenti consente di comprendere meglio il significato attribuito alla partecipazione ai fini della tutela della sicurezza nei luoghi di lavoro. La «partecipazione equilibrata» promossa dalla direttiva quadro del 1989 ha, infatti, assunto significati in parte diversi nei vari Stati membri, in virtù dei peculiari contesti giuridici e di discipline preesistenti.

(Rechtsmissbrauch): H. Hess, M. Worzalla, D. Glock, A. Nicolai, F.J. Rose, K. HuKe, BetrVG Kommentar, München, Luchterhand Verlag, 2014, p. 1139 ss.

123 P. BERG, \& 77 Durchführung gemeinsamer Beschlüsse, Betriebsvereinbarungen, in W. Däubler, M. KitTner, T. Klebe, P. Wedde (hrsg.), Betriebsverfassungsgesetz. Kommentar für die Praxis mit Wahlordnung und EBR-Gesetz, cit., p. 1490. La giurisprudenza ha però ammesso la possibilità per le parti di concordare una ultrattività anche per gli accordi aziendali volontari: v., ad esempio, BAG 28.04.1998, in NZA, 1998, p. 1348.

124 T. Klebe, $\& 87$ Mitbestimmungsrechte, in W. DäUbler, M. Kittner, T. KLeBE, P. WedDe (hrsg.), Betriebsverfassungsgesetz. Kommentar für die Praxis mit Wahlordnung und EBR-Gesetz, cit., p. 1692.

125 A sostegno dell'applicazione del limite di cui al § 77, comma 3, BetrVG anche nel caso di accordi aziendali disciplinati dal $\S 88$, cfr., in particolare, la pronuncia del BAG del 18.03.2010, in NZA-RR, 2011, p. 18. 
Deve essere, senza dubbio, rimarcato l'impatto positivo della presenza di strutture rappresentative dei lavoratori sulla effettività della tutela della salute e, dunque, sul tasso di infortuni sul lavoro ${ }^{126}$; come suggerito in un recente Approved Code of Practice and guidan$c e$, i lavoratori risultano maggiormente rispettosi delle misure di sicurezza se consapevoli del fatto che i loro rappresentanti sono stati consultati e hanno partecipato a crearle ${ }^{127}$.

I prestatori di lavoro sono, infatti, coloro che conoscono maggiormente i rischi connessi all'attività svolta e possono nel miglior modo contribuire insieme al management a sviluppare un sistema di sicurezza efficace. L'impresa o il singolo stabilimento diventano, in tal modo, un luogo di confronto e di scambio di conoscenze e pareri, con la possibilità di influenzare variamente le decisioni manageriali.

La legislazione italiana, come si è avuto modo di considerare, a differenza di altre esperienze (tra le quali ad esempio quella francese e spagnola), non ha predisposto un modello partecipativo aziendale che si fondi su istituti di consultazione e collaborazione ove entrambe le parti ivi rappresentate (lavoratori e datore di lavoro) possano agire insieme. Ad eccezione, infatti, degli organismi paritetici, presenti peraltro solo a livello territoriale, il d.lgs. n. 81/2008 non ha contemplato commissioni paritetiche o bilaterali con specifici compiti in materia di prevenzione dei rischi. A ciò sta, però, supplendo in alcune realtà la contrattazione collettiva, in ispecie aziendale, come già osservato.

Si può riflettere anche sul fatto che, in generale, l'esperienza dei comitati misti (in particolare in Francia e Spagna) è, tradizionalmente, slegata dalla presenza sindacale, per sua natura conflittuale. Il fine, almeno astrattamente, è e rimane quello della partecipazione collaborativa tra soggetti rappresentativi, non essendo determinante l'influenza delle organizzazioni sindacali sulla designazione o nomina dei componenti dei comitati. Questa caratteristica per la verità, come è stato chiarito, non è sempre rispettata nella prassi.

126 Quanto al Regno Unito cfr. Improving worker involvement. Improving health and safety, 2006, in www.hse.gov.uk/consult/index/htm.

127 ACOP and guidance, "Consulting workers on health and safety. Safety Representatives and Safety Committees Regulations», cit., p. 36. 
L'ordinamento interno, privo di una regolamentazione legislativa che istituisca e disciplini comitati con compiti precipui in materia, può apparire allora un po' arretrato rispetto ad altri, meno inclini a forme rivendicative o conflittuali e più propensi al perseguimento di interessi comuni.

L'impressione è in definitiva che, pur nella condivisione di principi e criteri comuni, il tema sia stato sviluppato più approfonditamente in altri Paesi membri, anche a causa di un contesto più favorevole di relazioni industriali, maggiormente predisposto cioè ad accogliere il monito della direttiva quadro del 1989. Così è senza dubbio quanto all'ordinamento tedesco, che rappresenta un termine di paragone ambizioso e molto lontano rispetto al sistema italiano, ma lo è, ad esempio, anche quanto al sistema francese che, pur in assenza di un meccanismo di codecisione, è caratterizzato dall'esistenza di organismi misti presenti all'interno dell'azienda o stabilimento con poteri propositivi e consultivi piuttosto significativi.

Degno di nota è anche il Comité pour la Prévention et la Protection au travail, organismo regolato nell'ordinamento belga dalla $L o i$ relative au bien-être des travailleurs lors de l'exécution de leur travail del 4 agosto 1996, cui sono riconosciute «prerogative che trascendono il tradizionale ambito della consultazione e informazione» ${ }^{128}$, con una competenza generale a «contribuire attivamente a tutto ciò che è necessario per promuovere il benessere dei lavoratori» (art. 65, Loi relative au bien-être des travailleurs); si presenta, quindi, come un «soggetto attivo coinvolto nelle politiche datoriali e strategiche nel campo della tutela della sicurezza» e nell'arricchimento delle misure di prevenzione dei rischi ${ }^{129}$.

128 Così F. Dorssemont, K. Naert, A. VAn Regenmortel, Well-Being at Work in Belgium: a Matter of Co-operation, in E. ALEs (ed.), Health and Safety at Work. European and Comparative Perspective, cit., p. 84.

129 F. Dorssemont, K. Naert, A. Van Regenmortel, Well-Being at Work in Belgium: a Matter of Co-operation, cit., p. 84. In proposito cfr., altresì, E. Ales, Occupational Health and Safety: A Comparative Perspective, in ID. (ed.), Health and Safety at Work. European and Comparative Perspective, cit., p. 431. Il Comité pour la Prévention et la Protection au travail è un istituto paritetico composto dal datore di lavoro e suoi rappresentanti in numero non superiore ai rappresentanti dei lavoratori, presente nelle imprese che occupano almeno cinquanta lavoratori (artt. 48-79 della Loi relative au bien-être des travailleurs). Si veda, inoltre, l'Arrêté royal du 3 mai 1999 relatif aux missions et au fonctionnement des comités pour la 
In sostanza, vigono altrove strumenti partecipativi alquanto articolati rispetto a quelli previsti nell'ordinamento italiano che, sotto questo profilo, può apparire manchevole.

A conclusione di queste riflessioni, si può brevemente tratteggiare quale possa essere il modello, se non da eguagliare, verso cui più realisticamente propendere nell'ottica di valorizzare il coinvolgimento degli stessi prestatori di lavoro. Da questo punto di vista non deve essere sottovalutato l'impatto positivo assunto dal Comité d'hygiène, de sécurité et des conditions de travail, divenuto nel tempo sempre più una sede di effettivo confronto ove i lavoratori, per il tramite delle loro rappresentanze, possono prendere parte alla gestione del sistema di sicurezza nel luogo di lavoro. Un istituto che, come acclarato in dottrina, dovrebbe essere salvaguardato e rafforzato $^{130}$.

In particolare l'esempio del citato Comité induce a riflettere sull'importanza, in ispecie nella materia della protezione della salute, di organismi bilaterali, non a composizione paritetica e per questo motivo, come già chiarito, effettivamente rappresentativi.

Così, pur rimanendo nel solco della mera «procedimentalizzazione» ${ }^{131}$, acquisirebbe maggior forza ed incisività il momento della consultazione dei lavoratori.

prévention et la protection au travail che, a completamento della Loi, ne specifica compiti e funzioni. Si deve precisare che la regolamentazione dell'istituto è contenuta nel nuovo «Code du bien-être au travail» del 28 aprile 2017.

130 Osservazioni critiche sulla possibilità di trasformazione o eliminazione del CHSCT sono state sollevate in particolare da L. Lerouge, P.-Y. Verkindt, Sauvegarder et renforcer le CHSCT: un enjeu majeur de santé au travail, cit., p. 365.

131 In ordine ai limiti di carattere procedimentale al potere datoriale cfr. C. Zoli, La tutela delle posizioni «strumentali» del lavoratore. Dagli interessi legittimi all'uso delle clausole generali, Milano, Giuffrè, 1988, p. 163 ss. 

CAPITOLO IV

\section{MODELLI DI ORGANIZZAZIONE E GESTIONE DELLA SICUREZZA}

Sommario: 1. La valorizzazione dell'adozione ed efficace attuazione dei modelli di organizzazione e gestione $e x$ art. 30 d.lgs. n. 81/2008. - 1.2. L'asseverazione da parte degli organismi paritetici. - 2. Tra volontarietà, onere e obbligo. -3 . Modelli organizzativi, buone prassi e codici etici: analogie, differenze e commistioni. - 4. Cenni sull'efficacia esimente della responsabilità amministrativa delle persone giuridiche in altri contesti nazionali.

1. La valorizzazione dell'adozione ed efficace attuazione dei modelli di organizzazione e gestione ex art. 30 d.lgs. n. 81/2008

Nell'ambito degli strumenti "volontari" messi a disposizione dal d.lgs. n. 81/2008, occorre, altresì, considerare i modelli di organizzazione e gestione idonei, ai sensi dell'art. 30, «ad avere efficacia esimente della responsabilità amministrativa delle persone giuridiche, delle società e delle associazioni anche prive di personalità giuridica» di cui al d.lgs. n. 231/2001, in conseguenza del verificarsi dei reati di omicidio colposo (art. 589 c.p.) e di lesioni colpose gravi o gravissime (art. 590 c.p.) commessi con violazione delle norme prevenzionistiche.

La rilevanza dei modelli organizzativi si rinviene nella possibilità di contribuire ad organizzare e gestire l'attività prevenzionistica 
all'interno dell'azienda con riguardo alle peculiarità ivi presenti' ${ }^{1}$; come espressamente disposto nelle previsioni normative non devono, infatti, essere trascurate la «natura» e le «dimensioni dell'organizzazione», nonché il «tipo di attività svolta», ossia le specificità della realtà aziendale (art. 30, comma 3, d.lgs. n. 81/2008). Si tratta, com'è stato definito, del «più utile» strumento indirizzato a «supportare nel migliore dei modi l'azione di quell'organizzazione aziendale a cui il legislatore ha affidato il compito di tutelare la salute e la sicurezza dei lavoratori» ${ }^{2}$.

L'attenzione è rivolta, allora, nuovamente all'organizzazione aziendale, rectius all'organizzazione del sistema di prevenzione entro cui, del resto, si inseriscono le stesse buone prassi già considerate $^{3}$. Compito fondamentale del datore di lavoro è proprio quello di «organizzare strategicamente l'attività preordinata all'osservanza dell'ingente mole di regole cautelari idonee ad impedire o ridurre al minimo i rischi di infortuni e malattie professionali $»^{4}$.

Come sottolineato, la maggior parte delle società ha ormai provveduto ad auto-organizzarsi, progettando modelli di gestione e, soprattutto, curandone l'«adeguamento dinamico» ${ }^{5}$. Le riflessioni che

1 Cfr. Trib. Milano 9 novembre 2004, in www.olympus. uniurb.it, ove è specificato che «i modelli, in quanto strumenti organizzativi della vita dell'ente, devono qualificarsi per la loro concreta e specifica efficacia e per la loro dinamicità» e «devono scaturire da una visione realistica ed economica dei fenomeni aziendali».

2 P. Pascucci, L'asseverazione dei modelli di organizzazione e di gestione, in I Working Papers di Olympus, n. 43/2015, p. 4 ss. Cfr., inoltre, ID., Prevenzione, organizzazione e formazione. A proposito di un recente libro sulla sicurezza sul lavoro, cit., p. 78 ss., il quale rimarca «l'esigenza di affiancare all'imprescindibile responsabilità individuale la responsabilità dell'organizzazione nel cui ambito si svolge l'azione individuale».

3 Si deve ricordare che tra gli interventi di miglioramento delle condizioni di sicurezza ed igiene sul lavoro richiesti al fine della riduzione del premio assicurativo, v'è l'adozione di un modello organizzativo e gestionale di cui all'art. 30, d.lgs. n. $81 / 2008$, oltreché la presentazione alla Commissione consultiva permanente di una buona prassi che sia stata validata precedentemente alla presentazione della domanda o l'adozione di una buona prassi tra quelle validate (cfr. l'iniziativa OT24).

4 V. Mongillo, Il dovere di adeguata organizzazione della sicurezza tra responsabilità penale individuale e responsabilità da reato dell'ente: alla ricerca di una plausibile differenziazione, in A.M. Stile, A. Fiorella, V. Mongillo (a cura di), Infortuni sul lavoro e doveri di adeguata organizzazione: dalla responsabilità penale individuale alla "colpa" dell'ente, Napoli, Jovene, 2014, p. 30.

5 C. Piergallini, Il modello organizzativo alla verifica della prassi, in Le Società, 2011, p. 47. In ordine alla «prospettiva diacronica» del modello organizzativo, implicante «un aggiornamento, ripetuto, affinché la strategia sia sempre pro- 
seguono non possono, appunto, prescindere da un esame concreto dei contenuti dei modelli, che facendo parte del sistema prevenzionistico adottato nell'azienda, si intrecciano con le buone prassi e, in un certo senso, con il portato della stessa valutazione dei rischi. È, infatti, vero, come chiarito, che «la valutazione dei rischi deve essere coordinata con l'adozione dei modelli», anche se «i due interventi non coincidono» ${ }^{6}$.

Si può, dunque, fin d'ora sottolineare che l'attività di valutazione dei rischi e il suo documento costituiscono il punto di riferimento per la costruzione di un valido modello di gestione della sicurezza. Così, il sistema (apparentemente volontario) di gestione della prevenzione dei rischi si affianca, integrandolo e completandolo, allo «schema gestionale obbligatorio» individuato nella procedura di valutazione dei rischi e di pianificazione degli interventi prevenzionistici, che consta già di per sé dell'impegno al costante miglioramento delle condizioni di salute, «secondo una logica di adempimento dinamico del dovere di sicurezza» ${ }^{7}$.

Occorre, in proposito, ricordare che l'art. 28, comma 2, lett. a, d.lgs. n. 81/2008, affida al datore di lavoro la scelta dei criteri di re-

porzionata alle minacce e alle migliori conoscenze tecniche» cfr. E. GRAGNOLI, $L a$ tutela della salute del lavoratore e la predisposizione dei modelli organizzativi, in D. Fondaroli, C. Zoli (a cura di), Modelli organizzativi ai sensi del D.Lgs. $n$. 231/2001 e tutela della salute e della sicurezza nei luoghi di lavoro, Torino, Giappichelli, 2014, p. 31.

6 E. Gragnoli, Artt. 28-30, in C. Zoli (a cura di), Principi comuni, in L. MonTUSCHI (dir.), La nuova sicurezza sul lavoro, cit., p. 409 ss., il quale ha precisato che «se la valutazione dei rischi è il perno dell'intera strategia di protezione della salute, propria del moderno diritto del lavoro, non è casuale il fatto che l'art. 30 sui modelli di organizzazione non sia collocato insieme all'art. 300 sulla revisione del d.lgs. n. 231/2001, ma, già sul piano visivo, sia messo a fianco degli artt. 28 e 29». Cfr., inoltre, le considerazioni di G. LosAPPIo, Organizzazione, colpa e sicurezza sul lavoro. Dosimetria dell'impresa e della colpa di organizzazione, in DSL, 2016, n. 1, p. 106 ss. In giurisprudenza cfr., in particolare, Trib. Trani, 11 gennaio 2010, in Dir. pen. proc., 2010, n. 7, p. 842, con nota di G. Amarelli, Morti sul lavoro: arriva la prima condanna per le società, e in Riv. trim. dir. pen. econ., 2010, p. 539, con nota di G. CHECCACCI, Responsabilità da reato degli enti per infortuni sul lavoro: la sentenza del Tribunale di Trani, ove è stata negata l'equiparazione tra il documento di valutazione dei rischi ex art. 28, d.lgs. n. 81/2008 ed il modello organizzativo di cui al d.lgs. n. 231/2001.

7 P. Tullini, I sistemi di gestione della prevenzione e della sicurezza sul lavoro, in DLRI, 2010, p. 404 ss. 
dazione del documento di valutazione dei rischi, «in modo da garantirne la completezza e l'idoneità quale strumento operativo di pianificazione degli interventi aziendali e di prevenzione». V'è, dunque, da chiedersi se la libertà nella scelta dei criteri non alluda anche alla possibilità di integrazione del contenuto stesso del documento; si potrebbe, infatti, trattare di un segnale, ancorché debole, della possibilità di integrazione dell'obbligo di sicurezza per mezzo di misure e modalità innovative non contemplate dal legislatore. Così, anche il contenuto del documento di valutazione dei rischi potrebbe, in un certo senso, intersecarsi con gli strumenti già presi in considerazione ossia con le buone prassi (cfr. il capitolo 1, paragrafo 7).

Attraverso il menzionato art. 30, d.lgs. n. 81/2008, è stato posto in essere una sorta di «meccanismo incentivante», destinato alle imprese che desiderano usufruire dell'esenzione dalle severe sanzioni di cui al d.lgs. n. 231/2001, con l'obiettivo di sostenere un moderno sistema prevenzionistico ${ }^{8}$.

$\mathrm{Al}$ fine di conseguire l'idoneità ad avere efficacia esimente della responsabilità amministrativa delle persone giuridiche, l'adozione ed efficace attuazione del modello organizzativo deve assicurare «un sistema aziendale per l'adempimento di tutti gli obblighi giuridici» indicati dallo stesso decreto e relativi alle principali attività prevenzionistiche, tra le quali in particolare quella di valutazione dei rischi e di predisposizione delle misure di protezione conseguenti (art. 30, comma 1 , lett. b) $)^{9}$. Ne consegue la «sinergia tra il modello organizzativo strumentale all'esenzione della responsabilità dell'ente e il sistema antinfortunistico disciplinato nel d.lgs. n. 81/2008» ${ }^{10}$.

8 P. PAscucci, La tutela della salute e della sicurezza dei lavoratori pubblici: cosa cambia dopo le riforme del 2008/2009?, in LPA, 2009, p. 617 ss.

9 In proposito cfr. E. Gragnoli, Artt. 28-30, in C. Zoli (a cura di), Principi comuni, in L. Montuschi (dir.), La nuova sicurezza sul lavoro, cit., p. 417, secondo il quale l'elenco di cui all'art. 30, comma 1, d.lgs. n. 81, «ha natura esemplificativa» ed il modello organizzativo «deve comportare una pianificazione strategica e la suddivisione delle competenze non con riguardo ai punti richiamati dall'art. $30,1^{\circ}$ co., ma agli interventi necessari in generale ai fini della prevenzione».

10 V. Mongillo, Il dovere di adeguata organizzazione della sicurezza tra responsabilità penale individuale e responsabilità da reato dell'ente: alla ricerca di una plausibile differenziazione, cit., p. 41. In ordine alle «interazioni» tra diritto del lavoro e d.lgs. n. 231/2001 v. S. MAINARDI, Codici etici nella prevenzione dei reati di lavoratori e collaboratori, modello organizzativo e sistema disciplinare, 
Secondo l'incerta previsione legislativa di cui all'art. 30, comma 5, d.lgs. n. 81/2008, «in sede di prima applicazione, i modelli di organizzazione aziendale definiti conformemente alle Linee guida UNI-INAIL per un sistema di gestione della salute e sicurezza sul lavoro (SGSL) del 28 settembre 2001 o al British Standard OHSAS 18001:2007 si presumono conformi ai requisiti di cui al presente articolo per le parti corrispondenti». Alla Commissione consultiva permanente è, inoltre, affidato il compito di indicare ulteriori modelli, senza però che nulla di più sia aggiunto.

Il meccanismo presuntivo appena richiamato ha destato non pochi dubbi in dottrina, ove pare prevalere una lettura che non intacca l'onere della prova a carico del datore di lavoro in merito alla rispondenza ai parametri indicati dal legislatore, né la facoltà del giudice di valutare inidoneo il modello adottato, nonostante la conformità alle suddette linee guida standardizzate ${ }^{11}$. Anche la giurisprudenza pare accogliere questa soluzione, affermando la necessità di svolgere comunque le «valutazioni volta per volta in concreto per stimare l'idoneità dei contenuti dei modelli organizzativi» ${ }^{12}$.

in D. Fondaroli, C. Zoli (a cura di), Modelli organizzativi ai sensi del D.Lgs. $n$. 231/2001 e tutela della salute e della sicurezza nei luoghi di lavoro, cit., p. 109 ss.

11 Cfr., in particolare, P. TuluINI, I sistemi di gestione della prevenzione e della sicurezza sul lavoro, cit., 406 ss. Il dibattito dottrinale ha riguardato, soprattutto, la configurabilità di una presunzione assoluta ovvero relativa: a favore della prima cfr. E. Amati, Art. 30, in G. Insolera (a cura di), Norme penali e processuali, in L. Montuschi (dir.), La nuova sicurezza sul lavoro, cit., p. 70; nonché R. LotTINI, I modelli di organizzazione e gestione, in F. Giunta, D. MicheletTI (a cura di), Il nuovo diritto penale della sicurezza nei luoghi di lavoro, Milano, Giuffrè, 2010, p. 191 ss.; contra F. D'ARCANGELo, La responsabilità da reato degli enti per gli infortuni sul lavoro, in Resp. amm. soc. enti, 2008, n. 2, p. 94, secondo cui «ove si ritenga che il sintagma normativo "si presumono conformi" enunci una presunzione assoluta (iuris et de iure) si perverrebbe ad una sostanziale clausola di esonero da responsabilità»; nonché T. VITARelli, Infortuni sul lavoro e responsabilità degli enti: un difficile equilibrio normativo, in RIDPP, 2009, p. 707. Sulla problematica cfr. P. IELo, Lesioni gravi, omicidi colposi aggravati dalla violazione della normativa antinfortunistica e responsabilità degli enti, in Resp. amm. soc. enti, 2008, p. 71; nonché G. D'AlessANDRO, Il modello di organizzazione, gestione e controllo (artt. 30-300 D.Lgs. n. 81/2008), in G. Natullo (a cura di), Salute e sicurezza sul lavoro, cit., p. 317 ss.

12 Trib. Brescia 11 marzo 2011, nonché Corte App. Brescia 23 giugno 2014: cfr. in proposito il commento di N. Mincato, F. Accardi, Idoneità ed effettiva attuazione del modello 231 ai fini sicuristici: la sentenza della Corte di Appello di Brescia del 23 giugno 2014, in Resp. amm. soc. enti, 2015, n. 2, p. 223 ss. 
Al di là dell'impreciso arco temporale entro cui la presunzione di conformità di cui all'art. 30, comma 5 , dovrebbe valere, altrettanto poco chiaro è sembrato come e quanto i modelli di organizzazione e gestione regolati dal decreto del 2008 debbano essere arricchiti ed integrati rispetto ai menzionati standard, soprattutto con riferimento ad un eventuale giudizio di responsabilità penale ${ }^{13}$.

Sul punto sono stati forniti chiarimenti con la circolare del Ministero del lavoro e delle politiche sociali dell'11 luglio 2011, che ha individuato quale necessario elemento aggiuntivo dei modelli regolati dall'art. 30, d.lgs. n. 81, «l'adozione di un sistema disciplinare idoneo a sanzionare il mancato rispetto delle misure indicate»; requisito, invece, non essenziale dei sistemi di gestione rispondenti alle linee guida ed agli standard richiamati. È, pertanto, necessario che siano predisposte in azienda procedure per individuare e sanzionare i comportamenti che possano favorire la commissione dei reati di cui all'art. 25-septies, d.lgs. n. $231 / 2001$, come sostituito dall'art. 300, d.lgs. n. 81/2008, e il mancato rispetto delle misure previste dal modello.

Destano, altresì, interesse le indicazioni fornite dalla Commissione consultiva permanente con il decreto ministeriale del 13 febbraio 2014, che ha recepito le procedure semplificate per l'adozione e l'efficace attuazione dei modelli organizzativi nelle piccole e medie imprese, ai sensi dell'art. 30, comma 5-bis, introdotto dal d.lgs. n. 106/2009. Sono state, in tal modo, individuate alcune indicazioni organizzative semplificate e di natura operativa, utili per le realtà di più ridotte dimensioni al fine della predisposizione ed efficace attuazione di un sistema aziendale idoneo a prevenire i reati previsti dal citato art. 25-septies, d.lgs. n. $231 / 2001^{14}$.

13 In proposito si veda V. Mongillo, Il giudizio di idoneità del modello di organizzazione ex d.lgs. n. 231/2001: incertezza dei parametri di riferimento e prospettive di soluzione, in Resp. amm. soc. enti, 2011, n. 3, p. 85, secondo cui l'intento racchiuso nell'art. 30, comma 5, è sembrato quello di «contenere l'interpretazione creatrice del giudice penale».

14 Come precisato nel d.m. del 13 febbraio 2014, «per piccole e medie imprese si fa riferimento a quelle definite dalla legislazione vigente»: Raccomandazione della Commissione europea 2003/361/CE del 6 maggio 2003, «relativa alla defini- 
A prescindere, tuttavia, da valutazioni concernenti le dimensioni aziendali, si può ritenere che i modelli organizzativi devono, in ogni caso, tendere ad andare oltre le previsioni contenute nel d.lgs. n. 81/2008 e contemplare un appropriato piano di miglioramento delle condizioni di salute e sicurezza, in modo non dissimile dalla stessa attività di valutazione dei rischi, volta alla garanzia del miglioramento nel tempo dei livelli di sicurezza.

Deve, cioè, essere programmata una strategia organizzativa che non si discosti dall'obiettivo sotteso allo stesso art. 2087 c.c.: il modello organizzativo è, infatti, uno strumento di ausilio che contribuisce a rendere trasparente e ad esplicitare la politica di prevenzione dei rischi adottata in azienda, ossia il processo di adempimento dell'obbligo di sicurezza.

\subsection{L'asseverazione da parte degli organismi paritetici}

Strettamente connesso con il menzionato art. 30 è l'art. 51, comma 3-bis, introdotto dal d.lgs. n. 106/2009, secondo cui gli organismi paritetici possono assumere un inedito e tuttora controverso ruolo di supporto alle imprese, attraverso l'asseverazione dell'adozione ed efficace attuazione dei modelli organizzativi, di cui, tra l'altro, gli organi di vigilanza possono tenere conto per la programmazione delle proprie attività ${ }^{15}$. Allo scopo di rilasciare, su richiesta delle imprese, la suddetta attestazione, sono istituite apposite commissioni paritetiche «tecnicamente competenti» (art. 51, comma 3-ter).

Occorre, anzitutto, interrogarsi in merito all'esatto significato da ascrivere al termine «asseverazione», che, pur implicando «la for-

zione delle microimprese, piccole e medie imprese», e decreto del Ministero delle attività produttive del 18 aprile 2005 su «Adeguamento alla disciplina comunitaria dei criteri di individuazione di piccole e medie imprese».

15 Cfr., tuttavia, le riflessioni di P. PAsCucC, L'asseverazione dei modelli di organizzazione e di gestione, cit. p. 11, secondo cui «se si legge attentamente la disposizione per come è formulata anche attraverso la punteggiatura, è facile osservare come l'oggetto della considerazione da parte degli organi di vigilanza non sarebbe tanto (e soltanto) l'asseverazione quanto piuttosto l'attestazione delle attività e dei servizi (tra cui appunto anche l'asseverazione)». 
mulazione di un giudizio fermo e deciso», deve essere tenuto distinto da quello di certificazione ${ }^{16}$.

Come precisato, si tratterebbe di una «specifica attestazione» della idoneità ed effettività dei modelli organizzativi, senza, tuttavia, che ciò escluda o limiti la verifica giudiziale al fine dell'esonero dalla responsabilità amministrativa di cui al d.lgs. n. 231/2001 ${ }^{17}$. Inoltre, non si deve dimenticare che l'asseverazione attiene solamente all'adozione ed efficace attuazione dei modelli di organizzazione e gestione, non invece all'adempimento in generale degli obblighi di prevenzione in capo al datore di lavoro ai sensi dell'art. 2087 c.c.; pare cioè che il legislatore indichi «un modello preferenziale per corrispondere ai precetti di legge privilegiando chi implementi la prevenzione aziendale entro la cornice del supporto e del controllo sociale offerto dalla pariteticità ${ }^{18}$.

Gli esiti dell'asseverazione non devono considerarsi vincolanti nemmeno nei confronti degli organi di vigilanza, nonostante la valutazione resa dagli organismi bilaterali possa essere «particolarmente attendibile sul piano tecnico» ${ }^{19}$. In sostanza tale «indicatore di liceità» sarebbe «liberamente valutabile dagli organi di vigilanza»e

16 M. LAI, Il sistema della rappresentanza e delle relazioni collettive, in P. PAScucCI (a cura di), Il d.lgs. n. 81/2008: due anni dopo. I "sistemi" del diritto della sicurezza sul lavoro, cit., p. 55; cfr., in tal senso, M. TIRABOSCHI, Il sistema di qualificazione delle imprese e dei lavoratori autonomi, in M. Tiraboschi, L. Fantini (a cura di), Il Testo Unico della salute e sicurezza sul lavoro dopo il correttivo (d.lgs. n. 106/2009), Milano, Giuffré, 2009, p. 128. Con riferimento al significato di «asseverazione», che corrisponde ad una affermazione «con solennità» ossia ad una «dichiarazione di particolare rilevanza formale e di particolare valore nei confronti dei terzi quanto alla verità ed alla affidabilità del contenuto» cfr. in giurisprudenza Cass. pen., 8 giugno 2011, n. 23072, in DeJure.

17 Cfr. L. Zoppoli, Il controllo collettivo sull'efficace attuazione del modello organizzativo per la sicurezza nei luoghi di lavoro, in D. Fondaroli, C. Zoli (a cura di), Modelli organizzativi ai sensi del D. Lgs. n. 231/2001 e tutela della salute e della sicurezza nei luoghi di lavoro, cit., p. 16; cfr., altresì, C. LAZZARI, Gli organismi paritetici nel decreto legislativo 9 aprile 2008, $n$. 81, cit., p. 12; M. D’APONTE, Obbligo di sicurezza e tutela dell'occupazione. Diritto alla salute e responsabilità dell'imprenditore dopo i d.lgss. 81/2008 e 106/2009, Torino, Giappichelli, 2012, p. 176.

18 P. Pascucci, L'asseverazione dei modelli di organizzazione e di gestione, cit., p. 15 ss.

19 C. Zoli, Art. 51, in ID. (a cura di), Principi comuni, cit., p. 534 ss., secondo il quale «il ruolo di supporto» svolto dagli organismi paritetici «non esonera i datori di lavoro dall'adozione di tutte le misure prescritte e non conferisce ad essi alcuna patente di legittimità»; in proposito v., altresì, M. RIccI, Gli organismi paritetici e il fondo di sostegno, in L. Zoppoli, P. PAscucci, G. NAtullo (a cura di), Le nuove regole per la salute e la sicurezza dei lavoratori, cit., p. 530 ss. 
«a maggior ragione dal giudice» ${ }^{20}$. La facoltà riconosciuta ai servizi ispettivi di programmare le proprie attività in considerazione anche di asseverate e idonee misure organizzative, quali parti integranti dell'obbligo di sicurezza, non è, peraltro, priva di fondamento.

Tale intervento, che rientra, in definitiva, tra i compiti di mera collaborazione attribuiti agli organismi paritetici, si affianca, in un certo senso, alla possibilità loro concessa, già presa in esame, di supportare le imprese anche nell'«individuazione di soluzioni tecniche ed organizzative», vale a dire di buone prassi aziendali, «dirette a garantire e migliorare la tutela della salute e sicurezza sul lavoro» (art. 51, comma 3, d.lgs. n. 81/2008) ${ }^{21}$.

Le scarne previsioni legislative, non toccate sul punto nemmeno dal recente d.lgs. n. 151/2015, attuativo della legge delega n. 183/2014, che si riferiscono all'attività, in prevalenza integrativa, di asseverazione dei modelli non contemplano affatto criteri univoci per tutte le imprese a cui gli organismi paritetici devono attenersi ${ }^{22}$. Deputata a fornire questi chiarimenti potrebbe essere, come sostenuto, la Commissione consultiva permanente ${ }^{23}$. Ciò pare quanto mai necessario, anche in considerazione delle indicazioni che viceversa sono state fornite dalla stessa Commissione per la richiesta di validazione delle buone prassi.

\section{Tra volontarietà, onere e obbligo}

Si deve riflettere, a questo punto, anche sulla scorta delle interpretazioni formulate dalla dottrina penalistica, sulla doverosità o

20 E. Amati, Art. 30, in G. Insolera (a cura di), Norme penali e processuali, in L. Montuschi (dir.), La nuova sicurezza sul lavoro, cit., p. 71.

21 Si veda il capitolo I, paragrafo 3.

22 Si vedano, al riguardo, gli «Indirizzi operativi per l'asseverazione nel settore delle costruzioni edili e di ingegneria civile», elaborati da UNI nel febbraio 2013, che intendono fornire «un'indicazione operativa nazionale per l'attività di asseverazione della corretta adozione e della efficace attuazione dei modelli di organizzazione e gestione della sicurezza di cui all'articolo 30, d.lgs. n. 81/2008, adottati dalle imprese edili e di ingegneria civile».

23 In proposito v. P. Pascucci, 3 agosto 2007-3 agosto 2009. Due anni di attività legislativa per la salute e la sicurezza dei lavoratori, Quaderni di Olympus-3, Fano, Aras Edizioni, 2011, p. 242. 
meno degli strumenti de quibus. Il dibattito, non ancora sopito, ha riguardato la possibilità di configurare l'esistenza di un onere o, forse più correttamente, di un vero obbligo all'adozione dei modelli di organizzazione e gestione.

Secondo le richiamate indicazioni della Commissione consultiva permanente contenute nel decreto ministeriale del 13 febbraio 2014, l'adozione del modello, «non essendo da considerarsi obbligatoria, deve essere valutata dalla Direzione aziendale in virtù delle proprie necessità ed esigenze gestionali ed organizzative». La decisione di dotarsi di un precipuo meccanismo organizzativo preventivo pare, così, affidata ad una valutazione di opportunità della singola impresa.

Pertanto, l'adozione del modello potrebbe considerarsi assolutamente facoltativa, almeno secondo le regole del d.lgs. n. 231/2001 (artt. 6 e 7) e la disciplina speciale dettata dal d.lgs. n. 81/2008 ${ }^{24}$. Si è, infatti, sostenuto che l'art. 30 di tale decreto identifichi unicamente «le misure preventive necessarie a conferire portata scriminante al modello medesimo» ${ }^{25}$. In mancanza di alcuna forma di «imposizione coattiva», rinvenibile nella normativa stessa, l'adozione è, dunque, intesa come «spontanea» ${ }^{26}$.

La scelta discrezionale di avvalersi di questo strumento, «in grado di eliminare o ridurre il rischio di commissione di illeciti da parte della società» ${ }^{27}$, che appunto non configura un preciso obbligo giuridico, è, però, volta a perseguire il risultato di evitare di incorrere nella responsabilità amministrativa. Viene in rilievo, dunque, la fattispecie dell'onere, che «si risolve in una necessitas», ma «di grado più attenuato» ${ }^{28}$.

24 Cfr., al riguardo, C. Piergallini, Paradigmatica dell'autocontrollo penale (dalla funzione alla struttura del "modello organizzativo" ex d.lgs. 231/2001), Relazione all'incontro di studio del Consiglio Superiore della Magistratura sul tema «Le tipologie di colpa penale tra teoria e prassi», 28-30 marzo 2011, in www.csm.it.; nonché, ex multis, F. D’ARCANGELo, I canoni di accertamento della idoneità del modello organizzativo nella giurisprudenza, in Resp. amm. soc. enti, 2011, p. 129 ss.

25 A. Bernasconi, Art. 6, in A. Presutti, A. Bernasconi, C. Fiorio, La responsabilità degli enti, Padova, Cedam, 2008, p. 116 ss., che sottolinea come la materia de qua sia contrassegnata dal «principio della discrezionalità nella adozione degli strumenti di compliance». V., al riguardo, le considerazioni di P. TuldinI, I sistemi di gestione della prevenzione e della sicurezza sul lavoro, cit., p. 406 ss.

26 Cass. pen., 23 giugno 2006, n. 32627, in Cass. pen., 2007, p. 4227.

27 F. D'Arcangelo, I canoni di accertamento della idoneità del modello organizzativo nella giurisprudenza, cit., p. 129 ss.

28 V. Durante, voce Onere, in Enc. Giur. Treccani, XXI, Roma, 1990, p. 3. 
L'adozione ed efficace attuazione «ante factum di un idoneo programma di prevenzione del rischio reato» è preordinata alla «promessa di esenzione dalla responsabilità» delle persone giuridiche $^{29}$. L'opzione del modello organizzativo è, quindi, finalizzata al precipuo scopo di ingenerare un effetto giuridico favorevole e di poter usufruire dei benefici previsti ${ }^{30}$.

La dottrina, prevalentemente penalistica, ha preferito tale tesi, posto che la figura dell'onere «esalterebbe, specie nell'art. 6, la rilevanza ad probationem del modello, finalizzato a non incorrere nella responsabilità amministrativa ${ }^{31}$.

Anche secondo un indirizzo giurisprudenziale «l'ente, che voglia esimersi da responsabilità nel caso dei reati previsti dall'art. 25-septies, d.lgs. n. 231/2001, ha l'onere di efficacemente attuare modelli di organizzazione e gestione che assicurino un sistema aziendale per l'adempimento di tutti gli obblighi giuridici», ai sensi del menzionato art. 30, d.lgs. n. $81 / 2008^{32}$.

Alcune opinioni discordanti propendono, tuttavia, verso l'obbligatorietà ${ }^{33}$.

29 V. MonglLlo, Il giudizio di idoneità del modello di organizzazione ex d.lgs. n. 231/2001: incertezza dei parametri di riferimento e prospettive di soluzione, in Resp. amm. soc. enti, 2011, p. 71 ss.

30 O. Di Giovine, Lineamenti sostanziali del nuovo illecito punitivo, in G. LatTANZi (a cura di), Reati e responsabilità degli enti, Milano, Giuffrè, 2010, p. 87 ss.; cfr., altresì, V. Durante, voce Onere, cit., p. 3, secondo cui «la violazione dell'onere non si manifesta in un atto rilevante per il diritto, ma nel mancato conseguimento di una data utilità».

31 C. Piergallini, Paradigmatica dell'autocontrollo penale (dalla funzione alla struttura del "modello organizzativo" ex d.lgs. 231/2001), cit., p. 9 ss. Sulla problematica cfr. approfonditamente A. Bernasconi, Art. 6, in A. Presutti, A. BernaSCONI, C. Fionio, La responsabilità degli enti, cit., p. 119 ss.; a favore di tale tesi cfr., altresì, F. Curi, Colpa di organizzazione ed impresa: tertium datur. La responsabilità degli enti alla luce del testo unico in materia di sicurezza sul lavoro, in EAD. (a cura di), Sicurezza nel lavoro. Colpa di organizzazione e impresa, Bologna, Bononia University Press, 2009, p. 143.

32 Così, ad esempio, Trib. Monza, 3 settembre 2012, in www.olympus.uniurb. $i t$; esclude che l'adozione del modello organizzativo configuri un obbligo per gli enti Trib. Tolmezzo, 3 febbraio 2012, ivi.

$33 \mathrm{Si}$ veda, in proposito, F. GiUnta, Il reato come rischio d'impresa e la colpevolezza dell'ente collettivo, in Analisi giuridica dell'economia, 2009, p. 243 ss., il quale, in merito al ruolo dei modelli di organizzazione e gestione, ha precisato come risulti difficile «negare che la loro adozione costituisca un vero e proprio obbligo»; nonché N. PISANI, Profili penalistici del testo unico sulla salute e sicurezza 
Quanto alla materia della sicurezza nei luoghi di lavoro, non si è mancato di precisare, infatti, che il «modello organizzativo progettato dall'art. 30 include le prescrizioni cautelari indirizzate dal t.u. al datore di lavoro», e la violazione del suo contenuto, in tal modo «predeterminato dalla legge», determina un'ipotesi di colpa specifi$\mathrm{ca}^{34}$.

Con particolare riferimento al diritto delle società è stato richiamato il principio di doverosa «adeguatezza degli assetti organizzativi», che grava sugli organi sociali ai sensi degli artt. 2381, commi 3 e 5 e 2403, comma 1 c.c.; l'obbligo di corretta amministrazione e di predisposizione di assetti organizzativi adeguati alla natura e alle dimensioni della società, coinvolgerebbe in tal modo il modello stesso, che necessariamente dovrà essere adottato, conseguendo alla sua mancata attivazione la responsabilità degli amministratori per «inadeguata attività amministrativa» ${ }^{35}$. Ciò che dal punto di vista del d.lgs. n. 231/2001 pare essere connotato come un onere, da una prospettiva civilistica assume, invece, «i tratti dell'obbligo per gli amministratori» ${ }^{36}$.

È, tuttavia, stato chiarito che si tratterebbe di «una sovrapposizione di piani», da tenere «rigorosamente distinti», ossia quello dell'ente e quello degli organi sociali con i loro doveri di corretta amministrazione $^{37}$. Il diritto delle società e il sistema della preven-

sui luoghi di lavoro, in Diritto penale e processo, 2008, n. 7, p. 834. Cfr. altresì D. Pulitanò, La responsabilità da reato degli enti: i criteri d'imputazione, in RIDPP, 2002, I, p. 431 ss., secondo il quale «la predisposizione di modelli organizzativi idonei a prevenire il reato dei sottoposti è un obbligo, ad un tempo, dei soggetti apicali e dell'ente che essi "impersonano"».

34 T. Vitarelli, Infortuni sul lavoro e responsabilità degli enti: un difficile equilibrio normativo, cit., p. 709 ss.

35 V. Trib. Milano, 13 febbraio 2008, n. 1774, in Giur. comm., II, 2009, p. 177. In merito al principio di adeguatezza cfr., in particolare, V. Buonocore, Adeguatezza, precauzione, gestione, responsabilità: chiose sull'art. 2381, commi terzo e quinto, del codice civile, in Giur. comm., 2006, I, p. 5 ss.

36 M. RaвiтTI, Modelli organizzativi e responsabilità degli enti: l'incerta figura dell'organismo di vigilanza, in Resp. amm. soc. enti, 2010, p. 27 ss.

37 N. Abriani, La responsabilità da reato degli enti: modelli di prevenzione e linee evolutive del diritto societario, in Analisi giuridica dell'economia, 2009, p. 194 ss. Al riguardo, cfr. F. D’Arcangelo, I canoni di accertamento della idoneità del modello organizzativo nella giurisprudenza, cit., p. 137, che parla di «aporia solo apparente» tra l'onere di adozione del modello ai sensi dell'art. 6, d.lgs. n. 
zione dei reati «hanno continuato a scorrere lungo percorsi tra loro paralleli»; manca, infatti, nelle disposizioni codicistiche, anche successivamente alla riforma del 2003, qualsiasi riferimento ai modelli di prevenzione, e il d.lgs. n. 231/2001 rimane estraneo alle novità introdotte nell'ordinamento societario ${ }^{38}$.

Vero è che se, quantomeno formalmente, non è del tutto ipotizzabile la configurabilità di un obbligo, l'onere in esame molto si avvicina, tuttavia, ad un atto dovuto, ad una «necessità per le Società che vogliano operare con la ragionevole certezza di non essere coinvolte nel meccanismo sanzionatorio» previsto dal d.lgs. n. $231 / 2001^{39}$. Si tratta, cioè, sostanzialmente di un obbligo. L'obbligatorietà non è, infatti, comminata espressamente dal legislatore, bensì deriva indirettamente dalle conseguenze favorevoli che lo stesso d.lgs. n. 81/2008 riconosce in caso di adozione ed efficace attuazione del modello organizzativo.

A differenza di quanto disposto in ordine all'obbligo di valutazione dei rischi, ove vi è responsabilità in ragione della violazione della regola di prevenzione (art. 55, comma 1, lett. a), a prescindere

231/2001 e i doveri di «adeguatezza organizzativa» posti dagli artt. 2381 e 2403 c.c.

38 N. ABRIANI, La responsabilità da reato degli enti: modelli di prevenzione e linee evolutive del diritto societario, cit., p. 187 ss.

39 In tal senso il modello di organizzazione e gestione di Megagest S.r.l., parte generale, aggiornato al 16 dicembre 2013. In merito alla «necessità che l'azienda adotti un modello di organizzazione e gestione» cfr. G. NATUllo, Sicurezza del lavoro e modelli organizzativi: brevi osservazioni su alcuni profili individuali e colletti$v i$, in D. Fondaroli, C. Zoli (a cura di), Modelli organizzativi ai sensi del D. Lgs. $n$. 231/2001 e tutela della salute e della sicurezza nei luoghi di lavoro, cit., p. 52 ss. In giurisprudenza cfr., al riguardo, Cass. pen., 17 settembre 2009, n. 36083, in DeJure; occorre, in particolare, ricordare la nota sentenza della Cassazione penale, S.U., 24 aprile 2014, n. 38343, in Cass. pen., 2015, p. 426, con nota di K. Summerer, $L a$ pronuncia delle Sezioni Unite sul caso Thyssen Krupp. Profili di tipicità e colpevolezza al confine tra dolo e colpa, ove è stato affermato che il legislatore «orientato dalla consapevolezza delle connotazioni criminologiche degli illeciti ispirati da organizzazioni complesse, ha inteso imporre a tali organismi l'obbligo di adottare le cautele necessarie a prevenire la commissione di alcuni reati, adottando iniziative di carattere organizzativo e gestionale. Tali accorgimenti vanno consacrati in un documento, un modello che individua i rischi e delinea le misure atte a contrastarli. Non aver ottemperato a tale obbligo fonda il rimprovero, la colpa d'organizzazione». Per un commento cfr. G. De Simone, Il caso ThyssenKrupp: la responsabilità delle persone giuridiche, in L. Foffani, D. Castronuovo, Casi di diritto penale dell'economia, Bologna, Il Mulino, 2015, p. 219 ss. 
dalla verificazione dell'evento lesivo, le conseguenze giuridiche della mancata adozione del modello di organizzazione e gestione sono apprezzabili solo se ricorre l'evento. Occorre che si crei l'occasione (ossia il reato) per verificare l'adozione di un efficace modello organizzativo, ma una volta che ciò sia stato appurato, si realizza l'effetto esimente della responsabilità "promesso" dal decreto del 2008.

3. Modelli organizzativi, buone prassi e codici etici: analogie, differenze e commistioni

L'aspetto più interessante, forse non del tutto indagato finora, concerne il contenuto, invero non facilmente individuabile dei modelli organizzativi atti a prevenire il verificarsi dei reati antinfortunistici.

Il modello di organizzazione e di gestione, affinché sia idoneo ad avere efficacia esimente, deve essere formato «da peculiari componenti, adottato ed efficacemente attuato, assicurando che il sistema aziendale preveda specifiche procedure e disposizioni interne in grado di garantire l'adempimento di tutti gli obblighi giuridici dettati dal d.lgs. n. $81 / 2008 »^{40}$.

Tale strumento, dovrebbe contraddistinguere e connotare ogni singola azienda, fungendo da contenitore di misure precipue riferentisi ai rischi ivi individuati, oltreché di soluzioni ad hoc, anche ulteriori rispetto alle regole già previste ed imposte dal legislatore, soprattutto a fronte dell'agognato obiettivo perseguito, ossia l'efficacia esimente della responsabilità amministrativa delle persone giuridiche, che corrisponde ad un quid pluris per l'impresa.

In realtà, dalla casistica esaminata, l'impressione è che il contenuto rimanga per lo più alquanto generico e unicamente descrittivo della struttura di prevenzione e protezione, con la mera riproposizione delle previsioni normative, senza alcuna attenzione alle criticità eventualmente presenti a livello aziendale.

40 Si veda, al riguardo, il documento illustrativo relativo al modello organizzativo di Alleanza Assicurazioni S.p.a. approvato il 5 maggio 2014, in www.alleanza.it. 
Normalmente il modello è suddiviso in una parte generale, ove sono definiti gli istituti ed i principi comunemente applicabili, ed in varie parti speciali, destinate a individuare criteri specifici in relazione alle attività esposte a specifici reati, tra i quali quelli di omicidio colposo e lesioni personali colpose gravi o gravissime (art. 25-septies, d.lgs. n. 231/2001). Ciascuna parte speciale comprende l'analisi normativa dei singoli reati richiamati dal d.lgs. n. 231/2001 e indica i principi ai quali devono ispirarsi i comportamenti nelle aree «a rischio reato».

Occorre, allora, valutare le misure prevenzionistiche contenute nella parte speciale dedicata ai reati con violazione delle norme sulla tutela della salute e sicurezza sul lavoro e riflettere sulle possibili commistioni in particolare con le buone prassi, già considerate. Infatti, benché i modelli, come accennato, tendano nella loro genericità a conformarsi al dettato legislativo, si possono riscontrare marginali esempi di soluzioni migliorative, ivi menzionate ed inserite, in prevalenza di tipo organizzativo.

Si può segnalare, anzitutto, l'istituzione della figura del «medico referente» con lo scopo «di migliorare e rendere più efficiente il servizio di sorveglianza sanitaria» ${ }^{41}$. Tale figura ha funzioni di coordinamento e di indirizzo dell'attività dei diversi medici competenti incaricati della sorveglianza sanitaria presso ogni unità produttiva $^{42}$. Tra i suoi compiti v'è quello di confrontarsi con i medici competenti «per l'applicazione della legislazione vigente», nonché di «redigere un protocollo sanitario unico di riferimento», di «offrire consulenze su eventuali contenziosi con le Autorità preposte ai controlli» e di «dare indicazioni su eventuali indagini diagnosti-

\footnotetext{
${ }^{41}$ Cfr. il modello di organizzazione e gestione, parte speciale, di Terna S.p.a., in www.terna.it.

42 La facoltà, lasciata alla libera discrezionalità del datore di lavoro, di nominare «più medici competenti, individuando tra essi un medico con funzioni di coordinamento» è, in verità, contemplata all'art. 39, comma 6, d.lgs. n. 81/2008, «nei casi di aziende con più unità produttive», «di gruppi d'imprese, nonché qualora la valutazione dei rischi ne evidenzi la necessità». In proposito cfr. TAR Abruzzo, Pescara sez. I, 21 giugno 2010, n. 705, in www.olympus. uniurb.it, ove è stato precisato che, secondo la citata previsione, una volta esercitata «la possibilità di nominare più medici», «debba necessariamente essere nominato tra di essi anche il medico con funzioni di coordinamento».
} 
che da effettuare sul territorio nazionale in relazione a particolari rischi ambientali» ${ }^{43}$.

Di non poco rilievo sono le conseguenze, dal punto di vista giuridico, derivanti dall'introduzione di questa figura appositamente incaricata dall'azienda, soprattutto con riguardo ai possibili profili di responsabilità connessi all'attività di coordinamento. In verità, gli specifici compiti di mediazione ed ausilio non dovrebbero intaccare la responsabilità di carattere esclusivo posta in capo ai soggetti individuati dal legislatore; sicché il medico coordinatore non assumerebbe una vera posizione di garanzia ai sensi del d.lgs. n. 81/2008.

Sempre per quanto concerne l'obbligo di sorveglianza sanitaria in azienda significativa e degna di nota è l'estensione del suo ambito di applicazione nei confronti della «totalità dei lavoratori subordinati», che pare, dunque, non limitato alle sole situazioni di rischio espressamente contemplate dalla legge (cfr. art. 41, d.lgs. n. $81 / 2008)^{44}$.

È rivolta, inoltre, attenzione all'obbligo formativo e informativo dei dipendenti, quale elemento essenziale per l'effettività del sistema di prevenzione dei rischi ${ }^{45}$. Con particolare riguardo alle esigenze dei neoassunti, è precisato che «in assenza di pregressa esperienza lavorativa e di adeguata qualificazione» è esclusa la possibilità per i lavoratori «di essere adibiti in autonomia ad attività ritenute più a rischio di infortuni, se non dopo l'acquisizione di un grado di professionalità idoneo $»^{46}$. La formazione in particolare di lavoratori giovani in fase di assunzione costituisce, tra l'altro, oggetto di buone prassi già validate da parte della Commissione consultiva permanente ${ }^{47}$.

${ }^{43}$ V. il citato modello di organizzazione e gestione, parte speciale, di Terna S.p.a.

44 In proposito si veda il modello di organizzazione, gestione e controllo, parte speciale, di Ferrari S.r.l., rivisto il 15 dicembre 2015, in www.ferraripiu.it.

45 Sia consentito rinviare a M. VINCIERI, Buone prassi e modelli di organizzazione e gestione nella tutela della sicurezza sul lavoro: distinzioni e possibili interferenze, in D. Fondaroli, C. Zoli (a cura di), Modelli organizzativi ai sensi del D.Lgs. n. 231/2001 e tutela della salute e della sicurezza nei luoghi di lavoro, cit., p. 80.

46 V. il modello di organizzazione e gestione di Megagest S.r.l., parte speciale; nonché il citato modello di organizzazione e gestione di Terna S.p.a.

47 Si veda la buona prassi presentata da ENEA Centro ricerche Casaccia e validata nel maggio 2013, dedicata agli ambienti di lavoro destinati alle attività di 
In alcuni modelli è, soprattutto, prevista la predisposizione di prove di verifica dell'apprendimento dei lavoratori discenti, nonostante non sia menzionata nel d.lgs. n. 81/2008, né successivamente richiesta, se non per i preposti ed i dirigenti, dall'Accordo Stato-Regioni del dicembre 2011 per la formazione dei lavoratori (ex art. 37, comma 2, d.lgs. n. 81/2008) ${ }^{48}$.

È, altresì, contemplata la previsione di apposite riunioni tra «le principali figure coinvolte nella gestione delle attività inerenti la salute e la sicurezza», in aggiunta rispetto all'obbligo di riunione periodica annuale ai sensi dell'art. 35, d.lgs. n. 81/2008 ${ }^{49}$.

Si deve, quindi, notare come il modello organizzativo diventi, per così dire, il contenitore di buone prassi finalizzate a migliorare la garanzia della protezione dei prestatori di lavoro. Non è escluso, cioè, che gli stessi modelli organizzativi accentuino alcune misure di protezione e ne individuino, finanche, di nuove ed ulteriori, in tal modo elevando i livelli di sicurezza in azienda.

Soluzioni innovative, di carattere organizzativo o procedurale, possono essere inserite fra le misure di sicurezza richiamate dai medesimi modelli, ritagliati sulle specifiche realtà aziendali, ed assumere, a fortiori, valore giuridico vincolante, tanto più in ragione della necessaria previsione di un sistema disciplinare idoneo a sanzionare

ricerca scientifica, ove è molto frequente l'ingresso di lavoratori giovani «con contratti cosiddetti atipici, spesso anche stranieri, legati ad interscambi culturali»; per la formazione di tali lavoratori è richiesta «un'attenzione particolare, preliminare alla fase di accesso agli ambienti di lavoro».

48 V. il modello di organizzazione e gestione di Megagest S.r.l., parte speciale; nonché il modello di organizzazione e gestione, parte speciale, di SIAE del luglio 2014, in www.siae.it. Cfr., in proposito, l'Accordo del 21 dicembre 2011 tra il Ministro del lavoro e delle politiche sociali, il Ministro della salute, le Regioni e le Province autonome di Trento e Bolzano per la formazione dei lavoratori e l'Accordo del 25 luglio 2012 della Conferenza permanente per i rapporti tra lo Stato, le Regioni e le Province Autonome di Trento e di Bolzano, che contemplano prove di valutazione e la verifica di apprendimento finale unicamente in ipotesi di formazione a distanza ossia in modalità $e$-Learning. Quanto all'individuazione della durata e dei contenuti minimi dei percorsi formativi per i responsabili e gli addetti dei servizi di prevenzione e protezione cfr. l’Accordo del 7 luglio 2016, tra il Governo, le Regioni e le Province Autonome di Trento e di Bolzano. In merito alla necessità di predisporre adeguati strumenti per la verifica dell'apprendimento dei lavoratori cfr., ad es., Cass. pen., 28 gennaio 2008, n. 4063, in www.olympus.uniurb.it; Cass. pen. 16 febbraio 2015, n. 6736, ivi.

49 Cfr. il modello di organizzazione, gestione e controllo di Ferrari S.r.l., cit. 
il mancato rispetto delle misure ivi indicate. I comportamenti posti in essere dai destinatari dei modelli in violazione della specifica parte speciale dedicata agli aspetti prevenzionistici si configurano, dunque, come «illeciti passibili di sanzioni» ${ }^{50}$.

Nonostante la diversità che li connota, prassi migliorative e modelli di organizzazione e gestione, a disposizione del datore di lavoro, possono quindi essere considerati strumentali all'adempimento dell'obbligo di sicurezza, in stretta connessione con la valutazione dei rischi. A conferma del legame esistente tra modello organizzativo e valutazione dei rischi, entrambi regolati nella medesima sezione II, capo III, titolo I, d.lgs. n. 81, v'è da considerare che, di frequente, l'analisi dei rischi è richiamata all'interno del modello stesso rectius della sua parte speciale.

Si deve, poi, precisare, che un «ruolo decisivo» ${ }^{51}$ nel valutare l'adeguatezza nel tempo del modello di organizzazione e gestione è attribuito al sistema interno di controllo, ai sensi dell'art. 30, comma 4, da collegare all'art. 6, comma 1, lett. b e d, d.lgs. n. 231/2001. L'organismo di vigilanza, la cui esatta configurazione ha suscitato un vivace dibattito in dottrina, è, infatti, precipuamente deputato a proporre eventuali integrazioni alle procedure già esistenti, così da migliorare l'azione di prevenzione in azienda, in considerazione di successive evoluzioni e mutazioni dell'organizzazione ${ }^{52}$. È stata, al riguardo, sottolineata sia «l'importanza cruciale» dell'organismo «all'interno di un adeguato sistema preventivo», sia la persistente

50 Cfr. il modello di organizzazione, gestione e controllo di Ferrari S.r.l., cit.

51 L. Fionillo, La responsabilità dell'impresa: profili civilistici, in A. Perulli, V. Brino (a cura di), Sicurezza sul lavoro. Il ruolo dell'impresa e la partecipazione attiva del lavoratore, cit., p. 64 ss.

52 In merito al problema della composizione dell'organismo di vigilanza cfr. L. Zoppoli, Il controllo collettivo sull'efficace attuazione del modello organizzativo per la sicurezza nei luoghi di lavoro, in D. Fondaroli, C. Zoli (a cura di), Modelli organizzativi ai sensi del D.Lgs. $n$. 231/2001 e tutela della salute e della sicurezza nei luoghi di lavoro, cit., p. 22 ss.; nonché G. MARRA, I modelli di organizzazione e di gestione e la responsabilità amministrativa degli enti per infortuni sul lavoro, in L. Zoppoli, P. Pascucci, G. Natullo (a cura di), Le nuove regole per la salute e la sicurezza dei lavoratori, cit., p. 601 ss. Quanto specificamente ai poteri di intervento dei componenti dell'organismo di vigilanza cfr. C. SANTORIELLo, Qual è la posizione dei componenti dell'OdV rispetto agli infortuni sul lavoro e le malattie professionali?, in Resp. amm. soc. enti, 2015, n. 2, p. 71 ss. 
«difficoltà nel trovare una precisa fisionomia, in punto di composizione, funzionamento e responsabilità» ${ }^{53}$.

Da questo punto di vista particolare rilevanza assumono le previsioni contenute nei modelli organizzativi che si riferiscono alla costante attività di vigilanza e di aggiornamento e che contribuiscono, insieme ad altri requisiti espressamente richiesti dal legislatore quali l'esistenza di un apparato sanzionatorio, a conferire effettività allo strumento in esame. Funzionali al controllo da parte dell'organismo di vigilanza sono i c.d. flussi informativi, che regolano le modalità di circolazione delle informazioni da parte dei soggetti della sicurezza all'interno dell'azienda e consentono di monitorare «le procedure di gestione della sicurezza e le notizie di eventuali anomalie accertate o presunte» ${ }^{54}$.

In particolare, all'organismo di vigilanza dovrà essere tempestivamente comunicata «ogni modifica della struttura organizzativa», al fine di consentire le opportune valutazioni che ne conseguono, nonché, «immediatamente», «ogni sospetto o evidenza di violazione fraudolenta delle procedure operative adottate per prevenire la commissione dei reati» ${ }^{55}$. Il modello organizzativo può contribuire, in tal modo, a consolidare e rafforzare «l'area della prevenzione antinfortunistica $»^{56}$.

53 V. Mongillo, L'organismo di vigilanza nel sistema della responsabilità da reato dell'ente: paradigmi di controllo, tendenze evolutive e implicazioni penalistiche, in Resp. amm. soc. enti, 2015, n. 4, p. 107.

54 Cfr. il modello di organizzazione e gestione di Megagest S.r.l., parte speciale, cit.; si veda, al riguardo, la circolare del Ministero del lavoro e delle politiche sociali dell'11 luglio 2011, contenente chiarimenti sul sistema di controllo, ove si precisa che tale sistema è attuato secondo quanto richiesto nell'art. 30, comma 4, d.lgs. n. 81/2008, «attraverso la combinazione di due processi che sono strategici per l'effettività e la conformità del sistema di gestione stesso», ossia il «monitoraggio/audit interno» ed il «riesame della Direzione».

55 Così il modello di organizzazione e gestione, parte speciale, di Finaval S.p.a., del 27 ottobre 2010; nonché il modello di organizzazione e gestione, parte specifica, di Pastorelli S.p.a. approvato il 31 gennaio 2013.

56 F. GIUNTA, I modelli di organizzazione e gestione nel settore antinfortunistico, in D. Fondaroli, C. Zoli (a cura di), Modelli organizzativi ai sensi del D.Lgs. n. 231/2001 e tutela della salute e della sicurezza nei luoghi di lavoro, cit., p. 9, secondo il quale il flusso informativo vale, altresì, «ad attivare un audit interno sul mancato evento, inteso a rimuovere le eventuali cause dell'inosservanza delle procedure cautelari». 
Si deve, infine, notare come normalmente sia incluso all'interno del modello stesso o da esso sia richiamato il codice etico, rappresentativo, insieme alle linee di condotta, dei principi generali di «trasparenza, correttezza, lealtà, cui si ispira lo svolgimento e la conduzione degli affari» ${ }^{57}$.

Senza dubbio il tema della protezione dei lavoratori nei luoghi di lavoro rileva anche sul piano etico ed è evocato dalle imprese che ambiscono ad essere distinte quali imprese di qualità e socialmente responsabili. Da questo punto di vista il riferimento è alla «centralità del contributo delle persone che lavorano con lealtà e reciproca fiducia» ed, in generale, alla «promozione della salute e sicurezza attraverso il miglioramento continuo dei sistemi di gestione ${ }^{58}$; si proclama, inoltre, l'impegno «a diffondere e consolidare una cultura della sicurezza», incrementando «la consapevolezza dei rischi» e sostenendo «comportamenti responsabili da parte di tutti i collaboratori $»^{59}$.

Tra i contenuti del codice etico in relazione ai reati colposi in materia di salute e sicurezza sul lavoro v'è, essenzialmente, il rispetto dei principi e dei criteri generali racchiusi nel d.lgs. n. 81/2008, già promossi nella direttiva quadro $89 / 391^{60}$.

Pare, in sostanza, trattarsi di mere enunciazioni dei fini ultimi da perseguire, di formule prive di elementi di originalità, ma la cui rilevanza dal punto di vista giuridico non è affatto trascurabile ${ }^{61}$. I

57 Si veda, ad esempio, il «Modello organizzativo 231. Documento di sintesi», del 15 marzo 2016, di Pirelli \& C. S.p.a., in www.pirelli.com. Cfr., inoltre, il codice etico di Finaval S.p.a., ove è espressamente previsto che «il codice etico è componente essenziale del modello di organizzazione e gestione». In merito all'adozione «di principi etici rilevanti ai fini della prevenzione dei reati ex D.lgs. n. 231/2001» cfr. le considerazioni di S. MaINARDI, Codici etici nella prevenzione dei reati di lavoratori e collaboratori, modello organizzativo e sistema disciplinare, cit., p. 112 ss.

58 Cfr. il «Modello organizzativo 231. Documento di sintesi», di Pirelli \& C. S.p.a., cit.

59 Si veda il codice etico di Pastorelli S.p.a.

60 Cfr. in particolare le «Linee guida per la costruzione dei modelli di organizzazione, gestione e controllo» approvate da Confindustria il 7 marzo 2002 ed aggiornate nel 2014.

61 Anche con riferimento alla natura giuridica dei codici di condotta sono state richiamate le considerazioni relative agli usi aziendali: cfr., in particolare, $\mathrm{M}$. Ferraresi, Responsabilità sociale dell'impresa e diritto del lavoro, Padova, Cedam, 
comportamenti etici sono, infatti, «inesorabilmente destinati ad un processo di giuridificazione»: le determinazioni unilaterali assumono, dunque, valore giuridico vincolante «del grado di buona fede e di diligenza che la società si obbliga a praticare» ${ }^{62}$.

Del resto, «l'etica d'impresa è da intendersi quale valore aggiunto che serve a smussare e levigare le asperità insite nel rapporto di lavoro, così esaltando il primato del diritto costituzionale alla salute ${ }^{63}$. Si può sostenere, cioè, che si tratti di manifestazioni di condotte volte a garantire beni ed interessi di rango costituzionale (art. 32 Cost.).

In definitiva, il codice etico è «un prodotto che si impone da sé» ${ }^{64}$, esprimendo nella maggior parte dei casi il rinnovato impegno, non contestabile, a quanto già la legislazione impone, dunque a prescindere dal consenso dei lavoratori, che appunto si presume.

2012, p. 92 ss. In merito all'effettività dei codici di condotta, quali strumenti di responsabilità sociale delle imprese v., ampiamente A. Perulli, La responsabilità sociale dell'impresa e i codici di condotta: modelli ed efficacia, in L. MontuschI, P. TulLinI (a cura di), Lavoro e responsabilità sociale dell'impresa, cit., p. 121 ss. Per una riflessione sul valore giuridico dei codici etici e di condotta nell'ordinamento spagnolo cfr. A. Baylos Grau, Códigos de conducta y buenas prácticas de las empresas en materia laboral. El problema de su exigibilidad jurídica, in E. LiLlo PeREZ (dir.), Aspectos económicos de la jurisdicción social, Consejo General del Poder Judicial, 2004, p. 265 ss.; nonché L. LóPEz Cumbre, La dimensión normativa nacional de la Responsabilidad Social de la Empresa, in EAD. (coord.), Autonomía y heteronomía en la Responsabilidad Social de la Empresa, Granada, Comares, 2012, p. 93.

62 N. IRTI, Due temi di governo societario (responsabilità «amministrativa»codici di autodisciplina), in G. Comm., 2003, p. 699, secondo cui «i codici etici nutrono l'incauta ambizione» di «nobilitare con i "valori" il valore del profitto» e tutto questo «costituisce fondamento di attese tutelate dal diritto».

63 L. Montuschi, Dai principi al sistema della sicurezza sul lavoro, in ID. (dir.), La nuova sicurezza sul lavoro, cit., p. 3. In ordine alle ragioni dell'adozione di codici etici o di condotta cfr. M. BARBERA, «Noi siamo quello che facciamo». Prassi ed etica dell'impresa post-fordista, in DLRI, 2014, p. 638, la quale sottolinea «il riconoscimento dell'impresa non solo come attore economico ma anche come attore politico».

64 L. Montuschi, Regolamenti aziendali, etica del lavoro e ricerca del consenso, in $A D L, 2001$, p. 423. 
4. Cenni sull'efficacia esimente della responsabilità amministrativa delle persone giuridiche in altri contesti nazionali

È interessante, a questo punto, chiedersi se simili strumenti di gestione della prevenzione in azienda, specificamente rivolti ad avere efficacia esimente della responsabilità amministrativa delle persone giuridiche, siano regolati anche in altri contesti nazionali. È, cioè, possibile comparare, seppur brevemente, la disciplina in materia di modelli organizzativi contemplata nell'art. 30, d.lgs. n. 81/2008?

In verità, tali studi comparatistici si prestano prevalentemente ad essere affrontati dalla dottrina penalistica che si è, in effetti, soffermata anche con uno sguardo rivolto altrove sui profili di responsabilità penale dell'ente in ipotesi di infortuni sul lavoro. Negli ordinamenti finora considerati, quali Spagna, Francia, Germania e Regno Unito, pare, infatti, essere per lo più assente una regolamentazione ad hoc contenuta nella relativa legislazione in materia di tutela della sicurezza sul lavoro. Anche da questo angolo visuale, come già anticipato rispetto alla esplicita previsione delle buone prassi, la normativa italiana, novellata di recente, tende pertanto a distinguersi.

Le alquanto scarne previsioni contenute nell'Arbeitsschutzgesetz, la già menzionata legge tedesca per il miglioramento della sicurezza e della salute dei lavoratori del 7 agosto 1996, non fanno riferimento alla responsabilità amministrativa delle persone giuridiche, né alla possibilità di istituire precipui strumenti di gestione che possano far conseguire una qual forma di esonero; i $\S \S 25$ e 26 dell'Arbeitsschutzgesetz delineano, invece, specificamente i profili di responsabilità individuale, penale e amministrativa ${ }^{65}$. La disciplina della responsabilità amministrativa degli enti collettivi in materia è dettata dall'Ordnungswidrigkeitengesetz, cioè la legge sulle violazioni amministrative, peraltro difficilmente assimilabile

65 In particolare per un commento ai $\S \S 25$ e 26 dell'Arbeitsschutzgesetz cfr. W. Kohte, U. Faber, K. Feldhoff (hrsg.), Gesamtes Arbeitsschutzrecht, cit., p. 230 ss. Cfr. C. Pelz, $\$ 25$ Bußgeldvorschriften, in N. Kollmer, D.T. Klindt, Arbeitsschutzgesetz Kommentar, München, Verlag C. H. Beck, 2011, p. 597 ss. 
«quanto a contenuti e scopo» al d.lgs. n. $231 / 2001^{66}$. Si è, infatti, affermato che ai sensi della menzionata legge ( $\$ 130)$ non è posto a carico degli enti «un vero e proprio dovere di auto-organizzazione, consistente nell'obbligo di dotarsi di modelli di organizzazione e gestione ${ }^{67}$.

Nell'ordinamento francese il code pénal ha regolato la responsabilità penale delle personnes morales (art. 121-2), estesa con la legge n. 2004-204 del 9 marzo 2004 a tutte le tipologie di reato, dunque anche alle ipotesi di reati colposi in violazione della normativa antinfortunistica ${ }^{68}$. È stato, in tal modo, reso possibile ricorrere a questo «strumento repressivo» anche con riguardo ai reati relativi alla salute e sicurezza sul lavoro regolati nel Code du travail, in passato esclusi «dal raggio d'azione della responsabilità delle persone giuridiche» ${ }^{69}$.

Tale responsabilità è, anzitutto, riconducibile, anche sulla scorta degli interventi giurisprudenziali, alla mancata o carente valuta-

66 S. Amari, Tutela della salute e sicurezza sul lavoro: responsabilità individuale e responsabilità dell'ente in Germania, in A.M. Stile, A. Fiorella, V. Mongillo (a cura di), Infortuni sul lavoro e doveri di adeguata organizzazione: dalla responsabilità penale individuale alla "colpa" dell'ente, cit., p. 452 ss.; in merito all'Ordnungswidrigkeitengesetz v., in particolare, C.E. PALIERO, voce Ordnungswidrigkeiten, in Digesto delle Discipline Penalistiche, Torino, Utet, 1995, vol. IX, p. 125.

67 F. GANDINI, Brevi cenni sulla responsabilità delle persone giuridiche in Germania, in Resp. amm. soc. enti, 2008, n. 4, p. 34 ss., secondo il quale «a differenza del modello previsto dal d.lgs. 231/2001, il $\S 130$ non definisce in modo puntuale quali siano le misure di salvaguardia ritenute necessarie per impedire la commissione di reati/illeciti amministrativi e prima ancora, quale sia il modulo organizzativo entro il quale queste misure devono essere inserite». In particolare il $\S$ 130 Ordnungswidrigkeitengesetz si riferisce alla responsabilità dovuta alla mancata adozione delle misure di sorveglianza necessarie per impedire la commissione di reati o illeciti amministrativi da parte del titolare dell'impresa. Tale norma deve essere letta insieme ai $\S \S 9$ e 30 OWiG. Per un commento cfr. G. De Simone, Persone giuridiche e responsabilità da reato. Profili storici, dogmatici e comparatistici, Pisa, Edizioni ETS, 2012, p. 310 ss. Quanto ai profili di responsabilità in materia cfr., inoltre, R. Aufhauser, Einführung (Vorbemerkung vor $\$ 1$ ), in R. Aufhauser, H. BrunhöBer, P. Igl, Arbeitssicherheitsgesetz. Handkommentar, Baden-Baden, Nomos, 2010, p. 29 ss.

68 Cfr. la Loi $n$. 2004-204 du 9 mars 2004 portant adaptation de la justice aux évolutions de la criminalité.

69 D. Castronuovo, La tutela della sicurezza del lavoro in Francia, Germania e Spagna, in M. Donini, D. CASTROnuovo (a cura di), La riforma dei reati contro la salute pubblica, Padova, Cedam, 2007, p. 117 ss. 
zione dei rischi o organizzazione del lavoro, finanche ritenendosi che si configuri una sorta di sovrapposizione tra le fattispecie contestate all'ente e le violazioni delle regole cautelari attribuite alla persona fisica ${ }^{70}$.

Rispetto a simili contesti si può, in ogni caso, sostenere che l'onere dell'adozione di modelli di organizzazione e gestione, con la finalità di esenzione dalla responsabilità amministrativa, sia in un certo senso una specificità propria del sistema prevenzionistico italiano, che arricchisce e completa, pur complicandola, la legislazione speciale di cui al d.lgs. n. 81/2008.

Quanto all'ordinamento spagnolo, il legislatore ha, di recente, disciplinato i profili di responsabilità penale delle persone giuridiche anche in relazione ai reati commessi in violazione delle norme a tutela della salute dei lavoratori. La Ley Orgánica 1/2015 del 30 marzo 2015 ha, infatti, introdotto modifiche incisive al código penal; tra le fattispecie delittuose imputabili alle persone giuridiche rientra il «delito contra los derechos de los trabajadores» e «contra la seguridad e higiene en el trabajo».

Al fine di queste brevi riflessioni assume particolare rilevanza proprio l'adozione di modelli di organizzazione e gestione, cui consegue l'esenzione della responsabilità penale delle persone giuridiche, purché contengano le misure di vigilanza e controllo idonee a prevenire i reati o a ridurne il rischio di commissione (art. 31-bis, código penal). In modo non dissimile a quanto disposto nell'ordinamento italiano dal d.lgs. n. 231/2001 (art. 6) sono, inoltre, specificamente indicati i requisiti dei modelli ossia,

70 E. VILLANI, La responsabilità penale delle persone giuridiche per gli infortuni sul lavoro in Francia, in A.M. Stile, A. Fiorella, V. Mongillo (a cura di), Infortuni sul lavoro e doveri di adeguata organizzazione: dalla responsabilità penale individuale alla "colpa" dell'ente, cit., p. 518; sui profili della responsabilità penale delle persone giuridiche nell'ordinamento francese v. A. COEuRET, Gouvernement des entreprises et reponsabilité pénale, in La gouvernabilité, Centre Universitaire de Recherches Administratives et Politiques de Picardie, Parigi, PUF, 1996, p. 126 ss.; nonché ID., Responsabilité pénale des personnes morales et groupe de sociétés, in La Semaine Juridique - Edition sociale, 2013, p. 1369 ss. Tra la dottrina italiana che si è occupata del tema cfr., in particolare, S. MANACORDA, voce Reato nel diritto penale francese, in Digesto delle Discipline Penalistiche, Torino, Utet, 1996, p. 312 ss.; C. DE MAGLIE, L'etica e il mercato. La responsabilità penale delle società, Milano, Giuffrè, 2002, p. 188 ss. 
in particolare, l'identificazione delle attività nel cui ambito possono essere commessi i reati, la previsione di obblighi di informazione nei confronti dell'organismo deputato a vigilare sul funzionamento e sull'osservanza dei modelli, nonché di un sistema disciplinare che sanzioni l'inadempimento delle misure stabilite nel modello ${ }^{71}$.

Non v'è traccia, però, all'interno della Ley de Prevención de Riesgos Laborales (31/1995), della possibilità di introdurre i suddetti modelli organizzativi in funzione di esonero dalla responsabilità, anche se in dottrina è stata messa in risalto la disciplina ivi contenuta della valutazione dei rischi, che «rappresenta uno dei pilastri della compliance» ${ }^{72}$. L'obbligo di apprestare un piano di prevenzione dei rischi è, senza alcun dubbio, tra i principi fondamentali alla base dell'azione preventiva del datore di lavoro $^{73}$. Occorre, altresì, ricordare che nella Ley sobre Infracciones y Sanciones en el Orden Social (LISOS) del 4 agosto 2000 sono contemplate apposite sanzioni in via amministrativa anche per gli enti collettivi ove non si sia provveduto a predisporre un piano di prevenzione, ai sensi dell'art. 12, comma 1, lett. a e b, e ove lo stesso piano sia carente perché, ad esempio, non aggiornato o rivisto.

71 Cfr. sul tema V. Gómez Martín, J. Navarro Massip, La responsabilidad penal para personas jurídicas en el Código Penal español: una visión panorámica tras la reforma de 2015, in Revista Aranzadi Doctrinal, 2016, n. 1, p. 5 ss.; nonché A. Pascual CADEna, El plan de prevención de riesgos penales y responsabilidad corporativa, Barcelona, Bosch, 2016, p. 161, secondo cui, tuttavia, le imprese, finora, non hanno assunto «la cultura empresarial del cumplimiento ético, normativo o preventivo»; si ravvisa, altresì, un «desconocimiento generalizado y confuso que existe sobre la responsabilidad penal de las personas jurídicas».

72 In tal senso v. A. Nieto MARTín, Compliance, responsabilità penale e tutela della sicurezza dei lavoratori in Spagna, in A.M. Stile, A. Fiorella, V. Mongillo (a cura di), Infortuni sul lavoro e doveri di adeguata organizzazione: dalla responsabilità penale individuale alla "colpa" dell'ente, cit., p. 504.

$73 \mathrm{Si}$ veda l'art. 16 della Ley de prevención de riesgos laborales in materia di «plan de prevención de riesgos laborales, evaluación de los riesgos y planificación de la actividad preventiva». Per un commento cfr., in particolare, F.A. GonZÁlez Díaz, M. Cos Egea, Artículo 16. Plan de prevención de riesgos laborales, evaluación de los riesgos y planificación de la actividad preventiva, in A.V. SEMPERE NAvarro (dir.), M. Cardenal Carro, I. Alzaga Ruiz (coord.), Comentarios a la Ley de Prevención de Riesgos Laborales, cit., p. 212 ss. 
Infine, quanto al Regno Unito v'è da ricordare che già il Robens Report del 1972 aveva posto l'accento sull'importanza di un sistema regolativo improntato alla self-regulation, piuttosto che allo schema tradizionale «command and control» ${ }^{74}$. In tale ordinamento, secondo le previsioni dell'Health and Safety at Work Act (HSWA) del 1974, successivamente modificato, a fronte dell'inadempimento degli obblighi a carico del datore di lavoro sono previsti regulatory offences, ovvero illeciti penali contravvenzionali, cui corrisponde una forma di responsabilità oggettiva (strict liability) ${ }^{75}$. V'è, poi, da menzionare il Corporate Manslaughter and Corporate Homicide Act del 2007, da cui si ricava proprio che l'organizzazione della sicurezza sul lavoro grava non solo sul datore di lavoro quanto soprattutto e direttamente sulla corporation $^{76}$.

Si può notare che nelle Explanatory Notes al Corporate Manslaughter and Corporate Homicide Act v'è un esplicito riferimento all'interessante istituto del «duty of care», inteso quale «obligation that an organisation has to take reasonable steps to protect a person's safety ${ }^{77}$; dunque un dovere di protezione nei confronti dei lavoratori che svolgono la loro attività nell'ambito dell'organizzazione aziendale. In definitiva, l'attenzione è rivolta alla predisposizione della strategia organizzativa, assumendo ri-

74 Cfr. le riflessioni di D. Walters, R. Johnstone, K. Frick, M. Quinlan, G. Baril-Gingras, A. Thébaud-Mony, Regulating Workplace Risks. A comparative study of inspection regimes in times of changes, Cheltenham, Edward Elgar Publishing, 2011, p. 54 ss.

75 Quanto ai profili di responsabilità v., in particolare, R. MatTHEws, J. AgeRos, Health and Safety Enforcement. Law and Practice, Oxford, Oxford University Press, 2010, p. 121 ss.; nonché B. BARRETT, R. Howells, Occupational health and safety law: texts and materials, cit., p. 219 ss.

76 Per un approfondimento sul tema cfr. V. Mongillo, Responsabilità individuale e responsabilità dell'ente per infortuni sul lavoro nel sistema penale del Regno Unito, in M. Stile, A. Fiorella, V. Mongillo (a cura di), Infortuni sul lavoro e doveri di adeguata organizzazione: dalla responsabilità penale individuale alla "colpa" dell'ente, cit., p. 479 ss.

77 Così Ministry of Justice, A Guide to the Corporate Manslaughter and corporate Homicide Act 2007, 2007, p. 8, ove si precisa che «these duties exist, for example, in respect of the systems of work and equipment used by employees, the condition of worksites and other premises occupied by an organisation and in relation to products or services supplied to customers». 
lievo come in concreto l'attività dell'organizzazione sia stata realizzata $^{78}$.

Tale «relevant duty of care» include espressamente il dovere di garantire un «sistema di lavoro sicuro», che tuttavia pare non coincidere, bensì aggiungersi, agli obblighi gravanti sul datore di lavoro in materia di prevenzione dei rischi regolati nell'Health and Safety at Work Act ${ }^{79}$.

78 Ministry of Justice, A Guide to the Corporate Manslaughter and corporate Homicide Act 2007, cit., p. 12.

79 Explanatory Notes al Corporate Manslaughter and Corporate Homicide Act. In proposito cfr. G. GENTILE, L'illecito colposo dell'ente collettivo. Riflessioni alla luce del Corporate Manslaughter, Torino, Giappichelli, 2009, p. 161, secondo il quale «gli obblighi che discendono dalla legislazione in materia di sicurezza sul lavoro non vanno confusi con il generale dovere di diligenza del datore di lavoro». 



\section{BiBLIOGRAFIA}

N. Abriani, La responsabilità da reato degli enti: modelli di prevenzione e linee evolutive del diritto societario, in Analisi giuridica dell'economia, 2009, p. 187.

M.C. Aguilar del Castillo, El Comité de Seguridad y Salud, Valencia, Tirant Lo Blanch, 2013.

M.C. Aguilar del Castillo, El Comité de Seguridad y Salud como órgano de participación de los trabajadores en la negociación colectiva, in J. Cabeza Pereiro, B. Fernández Docampo, Participación y acción sindical en la empresa, Albacete, Bomarzo, 2014, p. 113.

A. Alaimo, Germania, in B. Caruso, M. D’Antona, S. Sciarra (a cura di), Il diritto del lavoro nell'Unione europea. Francia, Germania, Gran Bretagna, Spagna, Napoli, Edizioni Scientifiche Italiane, 1995, vol. I, cap. III, p. 373.

A. Alaimo, L'eterno ritorno della partecipazione: il coinvolgimento dei lavoratori al tempo delle nuove regole sindacali, in DLM, 2014, p. 295.

P. Albi, Adempimento dell'obbligo di sicurezza e tutela della persona, in Il Codice Civile, Commentario fondato da P. Schlesinger, diretto da F.D. Busnelli, Milano, Giuffrè, 2008.

P. Albi, Sicurezza sul lavoro e sistema civile (2087 c.c.), in G. Natullo (a cura di), Salute e sicurezza sul lavoro, Milano, Utet Giuridica, 2015, p. 148.

P. Albi, La sicurezza sul lavoro e la cultura giuridica italiana tra vecchio e nuovo diritto, in DSL, 2016, n. 1, p. 83.

E. Ales, L'articolo 9 Statuto dei lavoratori alla luce della legislazione più recente in materia di salute e sicurezza: partecipazione o controllo?, in 
Studi in onore di Tiziano Treu. Lavoro, istituzioni, cambiamento sociale, II, Contratti di lavoro, Napoli, Jovene, 2011, p. 1049.

E. Ales, Occupational Health and Safety: A Comparative Perspective, in ID. (ed.), Health and Safety at Work. European and Comparative Perspective, Alphen aan den Rijn, Wolters Kluwer, 2013, p. 411.

E. Ales, Occupational Health and Safety: a European and Comparative Legal Perspective, in WP C.S.D.L.E. “Massimo D'Antona” .INT$120 / 2015$.

C.L. Alfonso Mellado, La reforma de la negociación colectiva en la Ley 3/2012: especial referencia a la negociación en la empresa, la estructura de la negociación y la inaplicación de los convenios, in Relaciones Laborales, 2013, n. 3, p. 15.

G. Alpa, voce Prassi, in Digesto delle Discipline Privatistiche, Sezione Civile, XIV, Torino, Utet, 1996, p. 138.

M. Álvarez de la Rosa, Ley y negociación colectiva en la regulación de la prevención de riesgos laborales, in Anales de la Facultad de Derecho, 2012, n. 29, p. 9.

S. Amari, Tutela della salute e sicurezza sul lavoro: responsabilità individuale e responsabilità dell'ente in Germania, in A.M. STILE, A. FiorelLA, V. Mongillo (a cura di), Infortuni sul lavoro e doveri di adeguata organizzazione: dalla responsabilità penale individuale alla "colpa" dell'ente, Napoli, Jovene, 2014, p. 445.

E. Amati, Art. 30, in G. Insolera (a cura di), Norme penali e processuali, in L. Montuschi (dir.), La nuova sicurezza sul lavoro, vol. III, Bologna, Zanichelli, 2011, p. 45.

L. Angelini, La sicurezza del lavoro nell'ordinamento europeo, in G. NATullo (a cura di), Salute e sicurezza sul lavoro, Milano, Utet Giuridica, 2015, p. 43.

J. Aparicio Tovar, Occupational Health and Safety in Spain: A Formidable Array of Employers' Liabilities, in E. Ales (ed.), Health and Safety at Work. European and Comparative Perspective, Alphen aan den Rijn, Wolters Kluwer, 2013, p. 249.

A. Apostoli, La forza propulsiva dell'art. 46 della Costituzione al di là della sua sostanziale inattuazione, in C. Zol (a cura di), Lavoro e impresa: la partecipazione dei lavoratori e le sue forme nel diritto italiano e comparato, Torino, Giappichelli, 2015, p. 15.

M.L. Arastey Sahún, Los riesgos psicosociales en la apreciación de los tribunales: algunas cuestiones sobre la aplicación práctica de la Directiva marco 1989/391/CEE, in Relaciones Laborales, 2012, p. 85. 
A. AsouInI, Usi legali e usi negoziali, in RDComm, 1944, I, p. 72.

A. Asouini, Integrazione del contratto con le «clausole d'uso», in Scritti giuridici, III, Padova, Cedam, 1961, p. 375.

R. Aufhauser, Einführung (Vorbemerkung vor $\S 1$ ), in R. Aufhauser, H. BrunhÖBer, P. IGL, Arbeitssicherheitsgesetz. Handkommentar, BadenBaden, Nomos, 2010, p. 19.

R. Aufhauser, $§ 9$ Zusammenarbeit mit dem Betriebsrat, in R. AufHauser, H. BrunhöBer, P. IgL, Arbeitssicherheitsgesetz. Handkommentar, Baden-Baden, Nomos, 2010, p. 103.

R. Aufhauser, $\$ 11$ Arbeitsschutzausschuß, in R. Aufhauser, H. BrunhöBer, P. IGL, Arbeitssicherheitsgesetz. Handkommentar, Baden-Baden, Nomos, 2010, p. 114.

G.G. BALANDI, Individuale e collettivo nella tutela della salute nei luoghi di lavoro: l'art. 9 dello statuto, in LD, 1990, n. 2, p. 219.

G.G. BaLANDI, Il contenuto dell'obbligo di sicurezza, in QDLRI, 1993, n. 14, p. 79.

R. Baldwin, T. Daintith, Harmonization and Hazard. Regulating Health and Safety in the European Workplace, London, Dordrecht, Boston, Graham \& Trotman, 1992.

L. BALESTRA, Introduzione al diritto dei contratti, Bologna, Il Mulino, 2015.

F. BAno, Diritto del lavoro e nuove tecniche di regolazione: il soft law, in LD, 2003, n. 1, p. 49.

M. BARbera, «Noi siamo quello che facciamo». Prassi ed etica dell'impresa post-fordista, in DLRI, 2014, p. 631.

B. Barrett, R. Howells, Occupational health and safety law: texts and materials, London, Cavendish Publishing, 2000.

F. BASENGHI, La legge delega in materia di sicurezza: alcuni spunti di indagine, in L. Montuschi (a cura di), Un diritto in evoluzione. Studi in onore di Yasuo Suwa, Milano, Giuffrè, 2007, p. 459.

A. Baylos Grau, Códigos de conducta y buenas prácticas de las empresas en materia laboral. El problema de su exigibilidad jurídica, in E. LILLo Perez (dir.), Aspectos económicos de la jurisdicción social, Consejo General del Poder Judicial, 2004, p. 247.

M. Bell, Occupational Health and Safety in the UK: At a Crossroads?, in E. Ales (ed.), Health and Safety at Work. European and Comparative Perspective, Alphen aan den Rijn, Wolters Kluwer, 2013, p. 375.

L. Bellard, Obiettivi, discipline e buone pratiche dei contratti di secondo livello: una breve rassegna, in L. MonTuschI (a cura di), Un diritto in evoluzione. Studi in onore di Yasuo Suwa, Milano, Giuffrè, 2007, p. 735. 
P. Bellocchi, Art. 2078 c.c., in G. Amoroso, V. Di Cerbo, A. Maresca, Diritto del lavoro, vol. I, Milano, Giuffrè, 2017, p. 742.

S. Bellumat, La gestione della salute e della sicurezza, in L. Noglen (a cura di), Ebav. Uno strumento delle parti sociali al servizio dell'artigianato veneto, Milano, Franco Angeli, 2014, p. 301.

G. BENEDETTI, Dal contratto al negozio unilaterale, Milano, Giuffrè, 1969.

P. Berg, $\$ 76$ Einigungsstelle, in W. Däubler, M. KitTner, T. Klebe, P. WedDe (hrsg.), Betriebsverfassungsgesetz. Kommentar für die Praxis mit Wahlordnung und EBR-Gesetz, Frankfurt am Main, Bund Verlag, 2014, p. 1417.

P. BERG, $\S 77$ Durchführung gemeinsamer Beschlüsse, Betriebsvereinbarungen, in W. Däubler, M. Kittner, T. Klebe, P. Wedde (hrsg.), Betriebsverfassungsgesetz. Kommentar für die Praxis mit Wahlordnung und EBR-Gesetz, Frankfurt am Main, Bund Verlag, 2014, p. 1465.

P. BERG, $\S 88$ Freiwillige Betriebsvereinbarungen, in W. Däubler, M. KITTNER, T. Klebe, P. WedDE (hrsg.), Betriebsverfassungsgesetz. Kommentar für die Praxis mit Wahlordnung und EBR-Gesetz, Frankfurt am Main, Bund Verlag, 2014, p. 1821.

A. BERnARDI, Sui rapporti tra diritto penale e soft law, in RIDPP, 2011, p. 536.

A. Bernasconi, Art. 6, in A. Presutti, A. Bernasconi, C. Fiorio, La responsabilità degli enti, Padova, Cedam, 2008, p. 109.

M. Biagi, Rappresentanza e democrazia in azienda. Profili di diritto sindacale comparato, Rimini, Maggioli, 1990.

M. BIAGI, Dalla nocività conflittuale alla sicurezza partecipata: relazioni industriali e ambiente di lavoro in Europa verso il 1992, in ID. (a cura di), Tutela dell'ambiente di lavoro e direttive Cee, Rimini, Maggioli, 1991, p. 123.

M. BIAGI, Cultura e istituti partecipativi delle relazioni industriali in Europa, in L'impresa al plurale. Quaderni della partecipazione, 2002, p. 291.

M. Bianca, Diritto civile, Milano, Giuffrè, 2002.

M. BIASI, Il nodo della partecipazione dei lavoratori in Italia. Evoluzioni e prospettive nel confronto con il modello tedesco ed europeo, Milano, Egea, 2013.

M. BIASI, La partecipazione dei lavoratori alla gestione e la Mitbestimmung tedesca, in C. Zoli (a cura di), Lavoro e impresa: la partecipazione dei lavoratori e le sue forme nel diritto italiano e comparato, Torino, Giappichelli, 2015, p. 33. 
A. Blume, U. Faber, $§ 5$ ArbSchG Beurteilung der Arbeitsbedingungen, in W. Kohte, U. Faber, K. Feldhoff (hrsg.), Gesamtes Arbeitsschutzrecht, Baden-Baden, Nomos, 2014, p. 205.

N. Bobbio, voce Consuetudine (teoria generale), in Enc. dir., XI, Milano, Giuffrè, 1961, p. 426.

G. Bolego, Art. 15, in C. Zoli (a cura di), Principi comuni, in L. MontuSCHI (dir.), La nuova sicurezza sul lavoro, Bologna, Zanichelli, 2011, vol. I, p. 140.

O. Bonard, Dentro al ginepraio della semplificazione. Salute e sicurezza sul lavoro nell'Italia che riparte, in RGL, 2016, n. 3, p. 593.

G. Borenfreund, Le comité d'entreprise: nouveaux enjeux, in Revue de droit du travail, 2015, p. 17.

M. BorZAGA, Il decentramento "controllato" della contrattazione collettiva nella Repubblica federale tedesca come antidoto alla crisi economica: realtà o mito?, in DLRI, 2015, p. 275.

M. Brollo, Uno sguardo d'assieme, in F. Carinci (a cura di), Da Pomigliano a Mirafiori: la cronaca si fa storia, Milano, Ipsoa, 2011, p. 117.

R.C. Browne, Safety and health at work: the Robens Report, in British Journal of Industrial Medicine, 1973, p. 87.

V. Buonocore, Adeguatezza, precauzione, gestione, responsabilità: chiose sull'art. 2381, commi terzo e quinto, del codice civile, in G. Comm., 2006, I, p. 5.

A. Cámara Botía, F.A. González Díaz, Derechos de participación y representación en materia de seguridad y salud laborales: un estudio sobre su aplicación, in Revista del Ministerio de Trabajo y Asuntos Sociales, 2004, n. 53, p. 219.

P. Campanella, Profili collettivi di tutela della salute e rappresentanza dei lavoratori per la sicurezza: disciplina legislativa, bilancio applicativo, prospettive di riforma, in $R G L, 2007$, supplemento al n. 2, p. 153.

P. CAmpanella, I rappresentanti dei lavoratori per la sicurezza, in L. Zoppoli, P. Pascucci, G. Natullo (a cura di), Le nuove regole per la salute e la sicurezza dei lavoratori, Milano, Ipsoa, 2010, p. 471.

P. Campanella, Benessere al lavoro, produttività dell'impresa, partecipazione dei lavoratori in tempo di crisi, in L. ANGELINI (a cura di), La prevenzione dei rischi da stress lavoro-correlato. Profili normativi e metodiche di valutazione, Atti del convegno Urbino 8 novembre 2013, in I Working Papers di Olympus, n. 31/2014, p. 57.

F. CARINCI, La partecipazione dei lavoratori alla gestione: la via italiana, 
in C. Assanti (a cura di), Dallo statuto dei diritti dei lavoratori alla dimensione sociale europea, Milano, Giuffrè, 1991, p. 203.

F. CARINCI, Habemus il testo unico per la sicurezza e la salute dei lavoratori: il d.lgs. 9 aprile 2008, n. 81, in ADL, 2008, nn. 4-5, p. 971.

B. Caruso, Introduzione, in B. Caruso, M. D’Antona, S. Sciarra (a cura di), Il diritto del lavoro nell'Unione europea. Francia, Germania, Gran Bretagna, Spagna, Napoli, Edizioni Scientifiche Italiane, 1995, vol. I, cap. II, p. 161.

B. CAruso, L'Europa, il diritto alla salute e l'ambiente di lavoro, in L. Montuschi (a cura di), Ambiente, Salute e Sicurezza. Per una gestione integrata dei rischi da lavoro, Torino, Giappichelli, 1997, p. 1.

B. CARUSO, «The bright side of the moon»: politiche del lavoro personalizzate e promozione del welfare occupazionale, in RIDL, 2016, n. 2, p. 177.

L. Castelvetri, Ma l'uso aziendale esiste?, in RIDL, 2000, II, p. 598.

D. Castronuovo, La tutela della sicurezza del lavoro in Francia, Germania e Spagna, in M. Donini, D. Castronuovo (a cura di), La riforma dei reati contro la salute pubblica, Padova, Cedam, 2007, p. 115.

D. Castronuovo, Responsabilità colposa e sicurezza del lavoro. Dalla colpa alle colpe, in M. Mantovani, F. Curi, S. Tordini Cagli, V. Torre, M. Caianiello (a cura di), Scritti in onore di Luigi Stortoni, Bologna, Bononia University Press, 2016, p. 165.

A. Coeuret, Gouvernement des entreprises et reponsabilité pénale, in La gouvernabilité, Centre Universitaire de Recherches Administratives et Politiques de Picardie, Parigi, PUF, 1996, p. 126.

A. Coeuret, Responsabilité pénale des personnes morales et groupe de sociétés, in La Semaine Juridique - Edition sociale, 2013, n. 39, p. 1369.

L. Corazza, Il nuovo conflitto collettivo. Clausole di tregua, conciliazione e arbitrato nel declino dello sciopero, Milano, Franco Angeli, 2013.

M. CoRTI, La partecipazione dei lavoratori. La cornice europea e l'esperienza comparata, Milano, Vita e Pensiero, 2012.

M. CoRTI, Le nuove frontiere della partecipazione dei lavoratori in Francia, in DRI, 2014, p. 284.

T. Coutrot, Le rôle des comités d'hygiène, de sécurité et des conditions de travail en France, in Travail et emploi, 2009, n. 117, p. 25.

A. Crosettr, voce Controlli amministrativi, in Digesto delle Discipline Pubblicistiche, Torino, Utet, 1989, p. 67.

J. CRuz Villalón, Impacto de las reformas laborales sobre la negociación colectiva, in Temas laborales, 2013, n. 120, p. 13.

F. CURI, Colpa di organizzazione ed impresa: tertium datur. La responsabi- 
lità degli enti alla luce del testo unico in materia di sicurezza sul lavoro, in EAD. (a cura di), Sicurezza nel lavoro. Colpa di organizzazione e impresa, Bologna, Bononia University Press, 2009, p. 127.

G. D'Alessandro, Il modello di organizzazione, gestione e controllo (artt. 30-300 D.Lgs. n. 81/2008), in G. NATUllo (a cura di), Salute e sicurezza sul lavoro, Milano, Utet Giuridica, 2015, p. 307.

M. D’Antona, voce Partecipazione dei lavoratori alla gestione delle imprese, in Enc. Giur. Treccani, vol. XXII, Roma, 1990, e in B. Caruso, S. ScIarra (a cura di), Massimo D'Antona Opere, vol. II, Scritti sul diritto sindacale, Milano, Giuffrè, 2000, p. 203.

M. D’Antona, Partecipazione, codeterminazione, contrattazione (temi per un diritto sindacale possibile), in RGL, 1992, p. 137, e in B. CARuso, S. Sciarra (a cura di), Massimo D’Antona Opere, vol. II, Scritti sul diritto sindacale, Milano, Giuffrè, 2000, p. 313.

M. D’Aponte, Obbligo di sicurezza e tutela dell'occupazione. Diritto alla salute e responsabilità dell'imprenditore dopo i d.lgss. 81/2008 e 106/2009, Torino, Giappichelli.

F. D’Arcangelo, La responsabilità da reato degli enti per gli infortuni sul lavoro, in Resp. amm. soc. enti, 2008, n. 2, p. 94.

F. D’Arcangelo, I canoni di accertamento della idoneità del modello organizzativo nella giurisprudenza, in Resp. amm. soc. enti, 2011, p. 129.

W. Däubler, Diritto sindacale e cogestione nella Germania Federale, ed. it. accresciuta a cura di M. Pedrazzoli, Milano, Franco Angeli, 1981.

W. DÄubler, Trade Union Rights at the Workplace in Germany, in R. BLANPAIN (ed.), Trade Union Rights at the Workplace, Alphen aan den Rijn, Wolters Kluwer, 2012, p. 61.

W. DÄUbler, La rappresentanza degli interessi dei lavoratori al di là della contrattazione collettiva, in LD, 2015, n. 1, p. 93.

R. De Luca Tamajo, Riforme (im)possibili nel diritto sindacale, in DLM, 2011, p. 1.

C. De Maglie, L'etica e il mercato. La responsabilità penale delle società, Milano, Giuffrè, 2002.

G. De Simone, Persone giuridiche e responsabilità da reato. Profili storici, dogmatici e comparatistici, Pisa, Edizioni ETS, 2012.

G. De Simone, Il caso ThyssenKrupp: la responsabilità delle persone giuridiche, in L. Foffani, D. Castronuovo, Casi di diritto penale dell'economia, Bologna, Il Mulino, 2015, p. 219. 
S. Deakin, G.S. Morris, Labour Law, Oxford and Portland, Hart Publishing, 2012.

O. DeinerT, Occupational Health and Safety in Germany: a Dual System in Change, in E. Ales (ed.), Health and Safety at Work. European and Comparative Perspective, Alphen aan den Rijn, Wolters Kluwer, 2013, p. 127.

E. Del Prato, voce Usi nel rapporto di lavoro, in Enc. Giur. Treccani, XXXII, Roma, 1994.

R. Del Punta, La rappresentanza sindacale e le rappresentanze dei lavoratori nel quadro degli sviluppi del diritto comunitario, in DRI, 1993, p. 183.

R. Del Punta, Contrattazione e partecipazione, in A. Baylos Grau, B. CAruso, M. D’Antona, S. Sciarra (a cura di), Dizionario di Diritto del Lavoro Comunitario, Bologna, Monduzzi Editore, 1996, p. 481.

R. Del Punta, Conclusioni II Sessione - "Consenso e dissenso nella rappresentanza e nel conflitto collettivo", in M. BARbera, A. Perulli (a cura di), Consenso, dissenso e rappresentanza: le nuove relazioni sindacali, Padova, Cedam, 2014, p. 269.

M. Despax, J. Rojot, J.P. Laborde, Labour law in France, Alphen aan den Rijn, Wolters Kluwer, 2011.

A. Deutsch, §§ 617-619. Besondere Pflichten des Dienstberechtigten, in M. Schmoeckel, J. Rückert, R. Zimmermann (hrsg.), Historisch-kritischer Kommentar zum BGB, III, Tübingen, Mohr Siebeck, 2013, p. 1251.

O. Di Giovine, Lineamenti sostanziali del nuovo illecito punitivo, in G. LATTANZi (a cura di), Reati e responsabilità degli enti, Milano, Giuffrè, 2010, p. 87.

F. Dorssemont, K. Naert, A. Van Regenmortel, Well-Being at Work in Belgium: a Matter of Co-operation, in E. Ales (ed.), Health and Safety at Work. European and Comparative Perspective, Alphen aan den Rijn, Wolters Kluwer, 2013, p. 67.

V. Durante, voce Onere, in Enc. Giur. Treccani, XXI, Roma, 1990.

U. Faber, W. KонтE, Vorbemerkungen zu §§ 20a, 20b ArbSchG, in W. Kohte, U. Faber, K. Feldhoff (hrsg.), Gesamtes Arbeitsschutzrecht, Baden-Baden, Nomos, 2014, p. 362.

U. Faber, Vorbemerkungen zu $\$ 87$ BetrVG, in W. Kohte, U. Faber, K. Feldhoff (hrsg.), Gesamtes Arbeitsschutzrecht, Baden-Baden, Nomos, 2014, p. 1259.

J.A. Fernández Avilés (dir.), La negociación colectiva en Prevención de Riesgos Psicosociales en el Trabajo, UGT-CEC, 2015.

J.A. Fernández Avilés, Reflexiones a los 20 años de vigencia de la Ley de 
Prevención de Riesgos Laborales: Mucho camino por recorrer, in Revista de Trabajo y Seguridad Social, 2016, p. 5.

M. Ferraresi, Responsabilità sociale dell'impresa e diritto del lavoro, Padova, Cedam, 2012.

L. Fiorillo, La responsabilità dell'impresa: profili civilistici, in A. PerulLI, V. BRino (a cura di), Sicurezza sul lavoro. Il ruolo dell'impresa e la partecipazione attiva del lavoratore, Padova, Cedam, 2012, p. 55.

M. Franzen, $\$ 3$ Tarifgebundenheit, in R. Müller-Glöge, U. Preis (hrsg.), Erfurter Kommentar zum Arbeitsrecht, München, Verlag C.H. Beck, 2010, p. 2619.

H.-W. Friedrich, $\S 618$, in H. Schliemann (hrsg.), Das Arbeitsrecht im BGB. Kommentar, Berlin, Walter de Gruyter, 2002, p. 459.

M. Fuchs, The Evolving Structure of Collective Bargaining in Europe 19902004. National Report Germany, in http://eprints.unifi.it/archive/00001160/.

L. GAETA, Infortuni sul lavoro e responsabilità civile. Alle origini del diritto del lavoro, Napoli, Edizioni Scientifiche Italiane, 1986.

L. Gaeta, Che cosa è oggi lo Statuto dei lavoratori?, in LD, 2010, n. 1, p. 49.

L. Galantino, La prassi aziendale, in DL, 1968, I, p. 222.

L. Galantino, Il contenuto dell'obbligo di sicurezza, in EAD. (a cura di), La sicurezza del lavoro. Commento al decreto legislativo 19 settembre 1994, n. 626, Milano, Giuffrè, 1996, p. 34.

F. Galgano, L’imprenditore, Bologna, Zanichelli, 1974.

F. GAlgano, L'impresa, il sistema economico, la partecipazione operaia, in C. Smuraglia, C. Assanti, F. Galgano, G. Ghezzi, La democrazia industriale. Il caso italiano, Roma, Editori Riuniti, 1980, p. 31.

F. Galgano, Il negozio giuridico, in Trattato di diritto civile e commerciale, già diretto da $\mathrm{A}$. Cicu, F. Messineo, L. Mengoni, continuato da $\mathrm{P}$. Schlesinger, Milano, Giuffrè, 2002.

F. GANDINI, Brevi cenni sulla responsabilità delle persone giuridiche in Germania, in Resp. amm. soc. enti, 2008, n. 4, p. 34.

J. García Blasco, A.L. de Val Tena, La negociación colectiva en materia de prevención de riesgos laborales, in J.L. Monereo Pérez, C. Molina Navarrete (dir.), S. Olarte Encabo, J.A. Fernández Avilés (coord.), Tratado de prevención de riesgos laborales. Teoría y práctica, Madrid, Tecnos, 2015, p. 660.

J.C. García QuiÑones, Sicurezza e salute sul lavoro: una prospettiva generale nel contesto dell'ordinamento giuridico spagnolo, in A. Peruld, 
V. Brino (a cura di), Sicurezza sul lavoro. Il ruolo dell'impresa e la partecipazione attiva del lavoratore, Padova, Cedam, 2012, p. 151.

M.G. Garofalo, La legislazione nel 1993-1994, in DLRI, 1995, p. 110.

M.G. Garofalo, Qualche osservazione su partecipazione e conflitto, in Rappresentanza collettiva dei lavoratori e diritti di partecipazione alla gestione delle imprese, Atti delle giornate di studio di diritto del lavoro, Lecce, 27-28 maggio 2005, Milano, Giuffrè, 2006, p. 357.

E. Garrido Pérez, La participación de los trabajadores en materia de prevención de riesgos laborales en la Ley 31/1995, in Relaciones Laborales, 1997, n. 2, p. 397.

A. Garrigues Giménez, Crisis y primer embate de la reforma de la negociación colectiva: la seguridad y salud laboral y la posible deriva hacia el dumping preventivo, in I Working Papers di Olympus, n. 4/2012.

G. Gentile, L'illecito colposo dell'ente collettivo. Riflessioni alla luce del Corporate Manslaughter, Torino, Giappichelli, 2009.

E. Ghera, Partecipazione dei lavoratori e contrattazione collettiva nell'impresa, in Impresa e Società, 1977, n. 5, p. 4.

J. GHESTIN, Rapport de synthèse, in Le rôle de la pratique dans la formation du droit. Travaux de l'Association H. Capitant, tome XXXIV, 1983, p. 3.

G. GHEzzI, La responsabilità contrattuale delle associazioni sindacali. La parte obbligatoria del contratto collettivo, Milano, Giuffrè, 1963.

G. Ghezzi, Art. 9, in G. Ghezzi, G.F. Mancini, L. Montuschi, U. RomagnoLI, Statuto dei diritti dei lavoratori, Bologna-Roma, Zanichelli-Il Foro italiano, 1972, p. 141.

G. Ghezzi, Art. 46, in G. Branca (a cura di), Commentario della Costituzione. Rapporti economici, tomo III, Bologna-Roma, Zanichelli-Il Foro italiano, 1980, p. 69.

G. GhezzI, La partecipazione dei lavoratori alla gestione delle imprese: il problema di una nuova "lettura" dell'art. 46 della Costituzione, in C. Smuraglia, C. Assanti, F. Galgano, G. Ghezzi, La democrazia industriale. Il caso italiano, Roma, Editori Riuniti, 1980, p. 96.

G. GHezzI, Una prospettiva di partecipazione conflittuale e non istituzionalizzata, in L'impresa al plurale. Quaderni della partecipazione, 1999, nn. 3-4, p. 195.

G. Giugni, Introduzione allo studio della autonomia collettiva, Milano, Giuffrè, 1960. 
G. Giugni, Mansioni e qualifica nel rapporto di lavoro, Napoli, Jovene, 1963.

G. Giugni, S. Sciarra, voce Contrattazione collettiva, in Novissimo Digesto Italiano, Appendice, vol. II, Torino, Utet, 1981, p. 606.

F. GIUNTA, Il reato come rischio d'impresa e la colpevolezza dell'ente collettivo, in Analisi giuridica dell'economia, 2009, p. 243.

F. GIUNTA, I modelli di organizzazione e gestione nel settore antinfortunistico, in D. Fondaroli, C. Zoli (a cura di), Modelli organizzativi ai sensi del D.Lgs. n. 231/2001 e tutela della salute e della sicurezza nei luoghi di lavoro, Torino, Giappichelli, 2014, p. 1.

B. Goldman, Rapport général, in Le rôle de la pratique dans la formation du droit. Travaux de l'Association H. Capitant, tome XXXIV, 1983, p. 164.

V. Gómez Martín, J. Navarro Massip, La responsabilidad penal para personas jurídicas en el Código Penal español: una visión panorámica tras la reforma de 2015, in Revista Aranzadi Doctrinal, 2016, n. 1, p. 5.

F.A. González Díaz, M. Cos Egea, Artículo 14. Derecho a la protección frente a los riesgos laborales, in A.V. Sempere NAVArro (dir.), M. CARdenal Carro, I. Alzaga Ruiz (coord.), Comentarios a la Ley de Prevención de Riesgos Laborales, Navarra, Aranzadi, 2010, p. 165.

F.A. González Díaz, M. Cos Egea, Artículo 16. Plan de prevención de riesgos laborales, evaluación de los riesgos y planificación de la actividad preventiva, in A.V. Sempere Navarro (dir.), M. Cardenal Carro, I. Alzaga Ruiz (coord.), Comentarios a la Ley de Prevención de Riesgos Laborales, Navarra, Aranzadi, 2010, p. 212.

E. González-Posada Martínez, La representación de los trabajadores en materia de salud laboral, in F. VAldés Dal-Ré, M.L. Molero MARAÑóN (dir.), La representación de los trabajadores en las nuevas organizaciones de empresa, Madrid, MTIN \& Fundación Largo Caballero, 2010, p. 183.

D. GotTARDI, La contrattazione collettiva tra destrutturazione e ri-regolazione, in $L D, 2016$, n. 4, p. 877.

E. Gragnoli, Artt. 28-30, in C. Zoli (a cura di), Principi comuni, in L. Montuschi (dir.), La nuova sicurezza sul lavoro, vol. I, Bologna, Zanichelli, 2011, p. 393.

E. Gragnoli, La tutela della salute del lavoratore e la predisposizione dei modelli organizzativi, in D. Fondarol, C. Zoli (a cura di), Modelli organizzativi ai sensi del D.Lgs. n. 231/2001 e tutela della salute e della sicurezza nei luoghi di lavoro, Torino, Giappichelli, 2014, p. 27. 
F. GuarRiello, I diritti di informazione e partecipazione, in M. Cinelli, G. Ferraro, O. Mazzotta (a cura di), Il nuovo mercato del lavoro, dalla riforma Fornero alla legge di stabilità 2013, Torino, Giappichelli, p. 751.

S. Guedes da Costa, E. Lafuma, Le CHSCT dans la décision d'organisation du travail, in Revue de droit du travail, 2010, p. 419.

S. HaRDY, Labour Law in Great Britain, Alphen aan den Rijn, Wolters Kluwer, 2012.

H. Hess, M. Worzalla, D. Glock, A. Nicolai, F.J. Rose, K. HuKe, BetrVG Kommentar, München, Luchterhand Verlag, 2014.

V. Howes, Workers' Involvement in Health and Safety Management and Beyond: The UK Case, in The International Journal of Comparative Labour Law and Industrial Relations, 2007, p. 245.

V. Howes, Duties and Liabilities under the Health and Safety at Work Act 1974: A Step Forward?, in Industrial Law Journal, 2009, vol. 38, n. 3, p. 306.

P. Ichino, Il contratto di lavoro, I, Milano, Giuffrè, 2000.

P. Ichino, La partecipazione dei lavoratori nell'impresa. Le ragioni di un ritardo, in RIDL, 2013, I, p. 861.

P. IELo, Lesioni gravi, omicidi colposi aggravati dalla violazione della normativa antinfortunistica e responsabilità degli enti, in Resp. amm. soc. enti, 2008, p. 71.

M.T. Igartua Miró, Sistema de prevención de riesgos laborales, Madrid, Tecnos, 2015.

M.T. Igartua Miró, Dos décadas de aplicación de la Ley 31/1995, de Prevención de Riesgos Laborales (LPRL): luces y sombras, in DSL, 2016, n. 1, p. 33.

N. IrTI, Due temi di governo societario (responsabilità «amministrativa»codici di autodisciplina), in G. Comm., 2003, p. 693.

O. Kahn-Freund, Sull'uso ed abuso del diritto comparato, in RTDPC, 1975, p. 785.

O. Kahn-Freund, Labour and the Law, London, Stevens \& Sons, 1977.

T. Kania, $\& 87$ Mitbestimmungsrechte, in R. Müller-Glöge, U. Preis (hrsg.), Erfurter Kommentar zum Arbeitsrecht, München, Verlag C.H. Beck, 2010, p. 1149.

T. KANIA, $\S 88$ Freiwillige Betriebsvereinbarungen, in R. MÜLLER-GLÖGE, U. PREIS (hrsg.), Erfurter Kommentar zum Arbeitsrecht, München, Verlag C.H. Beck, 2010, p. 1179.

T. KANIA, $\S 89$ Arbeits- und betrieblicher Umweltschutz, in R. MÜLLER- 
GlÖge, U. PReIs (hrsg.), Erfurter Kommentar zum Arbeitsrecht, München, Verlag C.H. Beck, 2010, p. 1180.

W. Kaskel, H. Dersch, Arbeitsrecht, Berlin-Heidelberg, Springer Verlag, 1957.

M. KitTner, R. PiePER, Arbeitsschutzrecht, Frankfurt am Main, Bund Verlag, 2002.

M. KitTNER, R. Pieper, Sicherheit und Gesundheitsschutz als Handlungsfeld des Betriebsrats, in Arbeitsrecht im Betrieb, 2005, p. 525.

T. Klebe, Dritter Abschnitt: Soziale Angelegenheiten, in W. Däubler, M. Kittner, T. Klebe, P. Wedde (hrsg.), Betriebsverfassungsgesetz. Kommentar für die Praxis mit Wahlordnung und EBR-Gesetz, Frankfurt am Main, Bund Verlag, 2014, p. 1763.

T. KLebe, $\& 87$ Mitbestimmungsrechte, in W. Däubler, M. Kittner, T. KleBE, P. Wedde (hrsg.), Betriebsverfassungsgesetz. Kommentar für die Praxis mit Wahlordnung und EBR-Gesetz, Frankfurt am Main, Bund Verlag, 2014, p. 1692.

D. KLoss, Occupational Health Law, Oxford, Wiley-Blackwell, 2010.

W. Koнte, Die Spontaneität des Südens und die Beständigkeit des Nordens. Impressionen zum kollektivvertraglichen Gesundheitsschutz, in R. Erd, R. Fabian, E. Kocher, E. Schmidt (hrsg.), Passion Arbeitsrecht. Erfahrungen einer unruhigen Generation. Liber amicorum Thomas Blanke, Baden-Baden, Nomos, 2009, p. 157.

W. Kонте, \& 88 Freiwillige Betriebsvereinbarungen, in F.J. Düwell (hrsg.), Betriebsverfassungsgesetz Handkommentar, Baden-Baden, Nomos, 2014, p. 1110.

M. LAI, La sicurezza del lavoro tra legge e contrattazione collettiva, Torino, Giappichelli, 2002.

M. LAI, Il sistema della rappresentanza e delle relazioni collettive, in P. PAscuccI (a cura di), Il d.lgs. n. 81/2008: due anni dopo. I "sistemi" del diritto della sicurezza sul lavoro, Atti dell'incontro di studio di Urbino (14-15 maggio 2010), Quaderni di Olympus-2, 2011, http://olympus. uniurb.it/, p. 52.

M. LAI, I soggetti collettivi (Rappresentanze per la sicurezza; Organismi paritetici): costituzione, prerogative, attività, in G. NATULLO (a cura di), Salute e sicurezza sul lavoro, Milano, Utet Giuridica, 2015, p. 795.

P. LAmbertucci, Tipologia, struttura e funzioni della contrattazione collettiva, in Trattato di diritto privato, diretto da M. Bessone, in F. CARINCI (a cura di), Il lavoro subordinato, tomo I, Il diritto sindacale, Torino, Giappichelli, 2007, p. 213. 
P. LAMBeRTUCCI, Usi aziendali e rapporto di lavoro: brevi note, in DML, 2009, p. 425.

P. LAMBertucci, Sicurezza sul lavoro e rischi psico-sociali: profili giuridici, in DLM, 2010, p. 347.

P. LAMBeRTUCCI, Gli usi aziendali tra contratto individuale e assetti collettivi dell'impresa: il "bilancio giurisprudenziale", in $L G, 2014$, p. 827.

P. Lambertucci, Conclusioni, in L. Angelini (a cura di), La prevenzione dei rischi da stress lavoro-correlato. Profili normativi e metodiche di valutazione, Atti del convegno Urbino 8 novembre 2013, I Working Papers di Olympus, n. 31/2014, p. 152.

A. LASSANDARI, Il contratto collettivo aziendale e decentrato, Milano, Giuffrè, 2001.

A. Lassandari, La contrattazione e il contratto collettivo, in P.G. Alleva, G. GHezzi (a cura di), Il Diritto del lavoro, Roma, Ediesse, 2002, fasc. 12.

A. Lassandari, Modello organizzativo: il sistema Ergo-Uas, in F. CARINCI (a cura di), Da Pomigliano a Mirafiori: la cronaca si fa storia, Milano, Ipsoa, 2011, p. 151.

A. Lassandari, Art. 2087 c.c., in O. Cagnasso, A. Vallebona (a cura di), Dell'impresa e del lavoro, Milano, Utet Giuridica, 2013, p. 181.

A. Lassandari, Divisione sindacale e "crescita di produttività e competitività", in $L D, 2013$, n. 2, p. 243.

A. LASSANDARI, La contrattazione integrativa, in M. MASCINI (a cura di), L'Annuario del lavoro 2014, Edizioni Il diario del lavoro, Roma, 2014, p. 107.

C. LAZZARI, Gli organismi paritetici nel decreto legislativo 9 aprile 2008, $n$. 81, in I Working Papers di Olympus, n. 21/2013.

$\mathrm{V}$. LeCCESE, Il diritto sindacale al tempo della crisi. Intervento eteronomo e profili di legittimità costituzionale, in DLRI, 2012, p. 479.

L. Lerouge, Le rôle du CHSCT reconnu par la jurisprudence, in Santé \& Travail, 2015, p. 29.

L. Lerouge, P.-Y. Verkindt, Sauvegarder et renforcer le CHSCT: un enjeu majeur de santé au travail, in Droit Social, 2015, p. 365.

S. Liebman, Individuale e collettivo nel contratto di lavoro, Milano, Giuffrè, 1993.

S. Liebman, Prassi aziendale e autonomia privata, in MGL, 1999, p. 45.

S. Liebman, Comportamenti unilaterali dell'imprenditore, usi aziendali e teoria delle fonti di regolamentazione del rapporto di lavoro, in $M G L$, 2000, p. 584. 
S. Liebman, Prassi aziendale e regolazione dei rapporti di lavoro nell'impresa: fonte consuetudinaria o prodotto dell'autonomia collettiva?, in $A D L, 2009$, n. 3, p. 704.

A. Lo Faro, Azione collettiva e tutela dell'ambiente di lavoro in Europa, in DLRI, 1991, p. 157.

A. Lo Faro, Germania, in B. Caruso, M. D'Antona, S. Sciarra (a cura di), Il diritto del lavoro nell'Unione europea. Francia, Germania, Gran Bretagna, Spagna, cap. II, vol. I, Napoli, Edizioni Scientifiche Italiane, 1995, p. 299.

G. Loiseau, L. Pécaut-Rivolier, P.-Y. Verkindt, Le Guide du Comité d'hygiène, de sécurité et des conditions de travail (CHSCT), Paris, Dalloz, 2015.

P. LoKIec, Droit du travail. Les relations collectives de travail, Paris, Presses Universitaires de France, 2011.

L. López Cumbre, La dimensión normativa nacional de la Responsabilidad Social de la Empresa, in EAD. (coord.), Autonomía y heteronomía en la Responsabilidad Social de la Empresa, Granada, Comares, 2012, p. 65.

G. Losappio, Organizzazione, colpa e sicurezza sul lavoro. Dosimetria dell'impresa e della colpa di organizzazione, in DSL, 2016, n. 1, p. 98.

R. LotTin, I modelli di organizzazione e gestione, in F. Giunta, D. MicheLETTI (a cura di), Il nuovo diritto penale della sicurezza nei luoghi di lavoro, Milano, Giuffrè, 2010, p. 167.

M. Lowisch, V. Rieble, Tarifvertragsgesetz. Kommentar, München, Vahlen, 2012.

F. LunARDon, Il contratto collettivo e le altre fonti (legge, usi, regolamenti), in Il sistema delle fonti nel diritto del lavoro, Atti delle giornate di studio di diritto del lavoro, Foggia-Baia delle Zagare, 25-26 maggio 2001, Milano, Giuffrè, 2002, p. 100.

M. Magnani, Responsabilità sociale d'impresa e ruolo delle relazioni sindacali, in L. MontuschI, P. TuluINI (a cura di), Lavoro e responsabilità sociale dell'impresa, Bologna, Zanichelli, 2006, p. 109.

S. MaINARDI, Codici etici nella prevenzione dei reati di lavoratori e collaboratori, modello organizzativo e sistema disciplinare, in D. FondARoli, C. Zoli (a cura di), Modelli organizzativi ai sensi del D.Lgs. $n$. 231/2001 e tutela della salute e della sicurezza nei luoghi di lavoro, Torino, Giappichelli, 2014, p. 107.

S. Mainardi, Le relazioni collettive nel "nuovo" diritto del lavoro, in Legge e contrattazione collettiva nel diritto del lavoro post-statutario, At- 
ti delle giornate di studio di diritto del lavoro, Napoli, 16-17 giugno 2016, Milano, Giuffrè, 2017, p. 181.

F. Malzani, Ambiente di lavoro e tutela della persona. Diritti e rimedi, Milano, Giuffrè, 2014.

S. Manacorda, voce Reato nel diritto penale francese, in Digesto delle Discipline Penalistiche, Torino, Utet, 1996, p. 304.

G.F. Mancini, Appunti di Diritto comparato del lavoro, Bologna, Riccardo Pàtron, 1954.

G.F. Mancini, La responsabilità contrattuale del prestatore di lavoro, Milano, Giuffrè, 1957.

M. MarazzA, Saggio sull'organizzazione del lavoro, Padova, Cedam, 2002.

G. Marini, voce Colpa II) Diritto Penale, in Enc. Giur. Treccani, VI, Roma, 1988.

L. MARIUcci, La contrattazione collettiva, Bologna, Il Mulino, 1985.

L. Mariucci, Per un nuovo rapporto fra conflitto e partecipazione, in LI, 1997, n. 10, p. 5.

L. MARIUCCI, È proprio un very bad text? Note critiche sulla riforma MontiFornero, in $L D, 2012$, n. 3-4, p. 415.

G. MARra, I modelli di organizzazione e di gestione e la responsabilità amministrativa degli enti per infortuni sul lavoro, in L. Zoppoli, P. PASCUCCI, G. NATUlLo (a cura di), Le nuove regole per la salute e la sicurezza dei lavoratori, Milano, Ipsoa, 2010, p. 601.

F. Martelloni, Inventario di sguardi sullo Statuto dei lavoratori, in LD, 2010, n. 4, p. 361.

R. Matthews, J. Ageros, Health and Safety Enforcement. Law and Practice, Oxford, Oxford University Press, 2010.

A. Mazeaud, Droit du travail, Paris, Montchrestien, 2010.

L. Meléndez Morillo-Velarde, La prevención de riesgos laborales en la negociación colectiva, Navarra, Aranzadi, 2004.

N. Mendoza Navas, Prevención de riesgos laborales: la regulación convencional, Albacete, Bomarzo, 2007.

N. Mendoza Navas, El tratamiento convencional de la salud y la seguridad en el trabajo, Granada, Comares, 2013.

L. Menghini, Lo Statuto dei lavoratori dall'autunno caldo del 1969 all'inverno gelido del 2010-2011, in Studi in onore di Tiziano Treu. Lavoro, istituzioni, cambiamento sociale, I, Il diritto del lavoro e i suoi interlocutori. Diritto sindacale e relazioni industriali, Napoli, Jovene, 2011, p. 313. 
L. Menghini, L'evoluzione degli strumenti giuridici volti a favorire l'effettività della prevenzione, in DSL, 2017, n. 2.

L. Mengoni, In tema di usi aziendali, in MGL, 1978, p. 471.

L. Mengoni, Democrazia industriale: conflitto o partecipazione?, in Mondoperaio, 1983, nn. 7-8, p. 25.

L. Mengoni, Recenti mutamenti nella struttura e nella gerarchia dell'impresa, in Diritto e valori, Bologna, Il Mulino, 1985, p. 335.

L. Mengoni, Diritto civile, in L. Mengoni, A. Proto Pisani, A. Orsi BattaGLINI, L'influenza del diritto del lavoro su diritto civile, diritto processuale civile, diritto amministrativo, in DLRI, 1990, p. 5.

J.R. Mercader Uguina, La ley de prevención de riesgos laborales, ventes años después, in Información Laboral, 2015, n. 8, p. 29.

F. Messineo, voce Contratto (dir. priv.), in Enc. dir., Milano, Giuffré, 1961, IX, p. 784.

V. Mongillo, Il giudizio di idoneità del modello di organizzazione ex d.lgs. n. 231/2001: incertezza dei parametri di riferimento e prospettive di soluzione, in Resp. amm. soc. enti, 2011, p. 69.

V. Mongillo, Il dovere di adeguata organizzazione della sicurezza tra responsabilità penale individuale e responsabilità da reato dell'ente: alla ricerca di una plausibile differenziazione, in A.M. STILE, A. FIoreLLA, V. Mongillo (a cura di), Infortuni sul lavoro e doveri di adeguata organizzazione: dalla responsabilità penale individuale alla "colpa" dell'ente, Napoli, Jovene, 2014, p. 19.

V. MongiLlo, Responsabilità individuale e responsabilità dell'ente per infortuni sul lavoro nel sistema penale del Regno Unito, in M. STILE, A. Fiorella, V. Mongillo (a cura di), Infortuni sul lavoro e doveri di adeguata organizzazione: dalla responsabilità penale individuale alla "colpa" dell'ente, Napoli, Jovene, 2014, p. 479.

V. Mongillo, L'organismo di vigilanza nel sistema della responsabilità da reato dell'ente: paradigmi di controllo, tendenze evolutive e implicazioni penalistiche, in Resp. amm. soc. enti, 2015, n. 4, p. 83.

A. Montoya Melgar, Estrategias y planes sobre prevención de riesgos laborales, in Nueva Revista Española de Derecho del Trabajo, 2015, p. 13.

L. Montuschi, Diritto alla salute e organizzazione del lavoro, Milano, Franco Angeli, 1976.

L. Montuschi, La tutela della salute e la normativa comunitaria: l'esperienza italiana, in M. BIAGI (a cura di), Tutela dell'ambiente di lavoro e direttive Cee, Rimini, Maggioli, 1991, p. 11. 
L. Montuschi, La sicurezza nei luoghi di lavoro ovvero l'arte del possibile, in $L D, 1995$, p. 405.

L. Montuschi, I principi generali del d.l.gs. n. 626 del 1994 (e le successive modifiche), in ID. (a cura di), Ambiente, salute e sicurezza. Per una gestione integrata dei rischi da lavoro, Torino, Giappichelli, 1997, p. 37.

L. Montuschi, voce Ambiente di lavoro, in Digesto delle Discipline Privatistiche, Sezione Commerciale, Torino, Utet, 2000, p. 7.

L. Montuschi, Regolamenti aziendali, etica del lavoro e ricerca del consenso, in $A D L, 2001$, I, p. 413.

L. Montuschi, L'incerto cammino della sicurezza del lavoro fra esigenze di tutela, onerosità e disordine normativo, in RGL, 2001, I, p. 501.

L. MontuschI, Aspettando la riforma: riflessioni sulla legge n. 229 del 2003 per il riassetto in materia di sicurezza sul lavoro, in ADL, 2004, p. 749.

L. Montuschi, La Corte Costituzionale e gli standard di sicurezza del lavoro, in $A D L, 2006$, n. 1 , p. 3.

L. Montuschi, Verso il testo unico sulla sicurezza del lavoro, in DLRI, 2007, p. 799.

L. Montuschi, Il sistema generale delle fonti giuslavoristiche, in M. PERSIANI (a cura di), Le fonti del diritto del lavoro, Trattato di diritto del lavoro, diretto da M. Persiani e F. Carinci, Padova, Cedam, 2010, vol. I, p. 395.

L. Montuschi, Dai principi al sistema della sicurezza sul lavoro, in ID. (dir.), La nuova sicurezza sul lavoro, Bologna, Zanichelli, 2011, vol. I, p. 1.

M.N. Moreno VIDA, Los derechos de participación y representación de los trabajadores en materia de prevención de riesgos. El Delegado de prevención, in J.L. Monereo Pérez, C. Molina Navarrete (dir.), S. Olarte Encabo, J.A. Fernández Avilés (coord.), Tratado de prevención de riesgos laborales. Teoría y práctica, Madrid, Tecnos, 2015, p. 632.

A. Morrone, Art. 1, in C. Zoli (a cura di), Principi comuni, in L. MontuSCHI (dir.), La nuova sicurezza sul lavoro, Bologna, Zanichelli, 2011, vol. I, p. 25.

M. NAPOLI, Per un progetto legislativo sulla partecipazione, in L'impresa al plurale. Quaderni della partecipazione, 2002, p. 307.

M. Napoli, Gli enti bilaterali nella prospettiva di riforma del mercato del lavoro, in Jus, 2003, p. 235.

M. NAPoli, Partecipazione e fonti del Diritto del lavoro, in Rappresentanza collettiva dei lavoratori e diritti di partecipazione alla gestione delle imprese, Atti delle giornate di studio di diritto del lavoro, Lecce, 27-28 maggio 2005, Milano, Giuffrè, 2006, p. 299. 
M. NAPOLI, La discussione parlamentare sulla partecipazione dei lavoratori alla gestione delle imprese: la via del sostegno tributario, in DRI, 2010, p. 72.

G. Natullo, Rappresentanze dei lavoratori per la sicurezza e rappresentanze sindacali in azienda, in ADL, 1997, p. 205.

G. Natullo, Riassetto normativo e sistema delle fonti, in G. Natullo, R. Santucci (a cura di), Ambiente e sicurezza sul lavoro. Quali tutele in vista del Testo Unico?, Milano, Franco Angeli, 2008, p. 19.

G. Natullo, I ruoli delle Regioni nel disegno del d.lgs. n. 81/2008, in P. Pascucci (a cura di), Il d.lgs. n. 81/2008: due anni dopo. I "sistemi" del diritto della sicurezza sul lavoro, Quaderni di Olympus-2, 2011, http://olympus.uniurb.it/, p. 108.

G. NATUllo, Sicurezza del lavoro e modelli organizzativi: brevi osservazioni su alcuni profili individuali e collettivi, in D. FonDaroli, C. Zoli (a cura di), Modelli organizzativi ai sensi del D.Lgs. n. 231/2001 e tutela della salute e della sicurezza nei luoghi di lavoro, Torino, Giappichelli, 2014, p. 52.

G. Natullo, Il quadro normativo dal Codice civile al Codice della sicurezza sul lavoro. Dalla Massima Sicurezza possibile alla Massima Sicurezza effettivamente applicata?, in ID. (a cura di), Salute e sicurezza sul lavoro, Milano, Utet Giuridica, 2015, p. 5.

F. Navarro Nieto, El tratamiento de la prevención de riesgos laborales en la negociación colectiva, in Temas Laborales, 2004, p. 11.

A.C. Neal, The European Framework Directive on the Health and Safety of Workers: Challenges for the United Kingdom?, in The International Journal of Comparative Labour Law and Industrial Relations, 1990, vol. 6, p. 80.

U. NICKEL, C. ReIK, Unternehmen und Betriebsrat zusammen für die Gesundheit. Gemeinsam Verantwortung tragen, in Arbeit und Arbeitsrecht. Sonderausgabe, 2014, p. 39.

A. Nieto Martín, Compliance, responsabilità penale e tutela della sicurezza dei lavoratori in Spagna, in A.M. Stile, A. Fiorella, V. Mongillo (a cura di), Infortuni sul lavoro e doveri di adeguata organizzazione: dalla responsabilità penale individuale alla "colpa" dell'ente, Napoli, Jovene, 2014, p. 501.

D. Nitsche, $\& 87$ BetrVG Mitbestimmungsrechte, in W. Kohte, U. Faber, K. Feldhoff (hrsg.), Gesamtes Arbeitsschutzrecht, Baden-Baden, Nomos, 2014, p. 1305.

L. NoGLer, Gli strumenti di «commercializzazione» del diritto del lavoro: bilancio sociale, certificazione etica e marchio di qualità sociale, in 
L. Montuschi, P. TulLinI (a cura di), Lavoro e responsabilità sociale dell'impresa, Bologna, Zanichelli, 2006, p. 143.

L. Nogler (a cura di), Gli enti bilaterali dell'artigianato tra neo-centralismo ed esigenze di sviluppo, Milano, Franco Angeli, 2014.

R. Nunin, Lavoro femminile e tutela della salute e della sicurezza: nuovi scenari per una prospettiva di genere dopo il d.lgs. $n$. 81/2008, in RDSS, 2011, n. 2, p. 383.

P. Olivelli, La partecipazione dei lavoratori tra diritto comunitario e ordinamento italiano, in DRI, 2010, p. 37.

P. Olivelli, G. Ciocca, La partecipazione del sindacato in generale, in F. Lunardon (a cura di), Conflitto, concertazione e partecipazione, Trattato di diritto del lavoro, diretto da M. Persiani, F. Carinci, vol. III, Padova, Cedam, 2011, p. 773.

A. Palermo, voce Omologazione, in Novissimo Digesto Italiano, vol. XI, Torino, Utet, 1982, p. 905.

C.E. Paliero, voce Ordnungswidrigkeiten, in Digesto delle Discipline Penalistiche, vol. IX, Torino, Utet, 1995, p. 125.

A. Pascual Cadena, El plan de prevención de riesgos penales y responsabilidad corporativa, Barcelona, Bosch, 2016.

P. Pascucci, Dopo la legge n. 123 del 2007. Prime osservazioni sul Titolo I del d.lgs. 9 aprile 2008, n. 81 in materia di tutela della salute e della sicurezza nei luoghi di lavoro, Quaderni di Olympus-1, Pesaro, edizioni studio@lfa, 2008.

P. PAscucci, La tutela della salute e della sicurezza dei lavoratori pubblici: cosa cambia dopo le riforme del 2008/2009?, in LPA, 2009, p. 553.

P. Pascucci, A. Andreani, L'estinzione agevolata degli illeciti amministrativi e il potere di disposizione, in L. Zoppoli, P. Pascucci, G. Natullo (a cura di), Le nuove regole per la salute e la sicurezza dei lavoratori, Milano, Ipsoa, 2010, p. 641.

P. PAscucci, Salute e sicurezza: dalle rappresentanze dell'art. 9 ai rappresentanti del d.lgs. n. 81/08, in DLM, 2010, n. 3, p. 663.

P. PAscucci, 3 agosto 2007-3 agosto 2009. Due anni di attività legislativa per la salute e la sicurezza dei lavoratori, Quaderni di Olympus-3, Fano, Aras Edizioni, 2011.

P. PAscucci, Introduzione, in L. AngelinI (a cura di), La prevenzione dei rischi da stress lavoro-correlato. Profili normativi e metodiche di valutazione, Atti del Convegno Urbino 8 novembre 2013, in I Working Papers di Olympus, n. 31/2014, p. 6.

P. PAscucci, La tutela della salute e della sicurezza sul lavoro: il titolo I 
del d.lgs. n. 81/2008, Quaderni di Olympus-4, Fano, Aras Edizioni, 2014.

P. PAscucci, L'asseverazione dei modelli di organizzazione e di gestione, in I Working Papers di Olympus, n. 43/2015.

P. PAscucci, Prevenzione, organizzazione e formazione. A proposito di un recente libro sulla sicurezza sul lavoro, in DSL, 2016, n. 1, p. 64.

M. Pedrazzoli, voce Democrazia industriale, in Digesto delle Discipline Privatistiche, Sezione Commerciale, Torino, Utet, 1989, p. 241.

M. Pedrazzoli, Alternative italiane sulla partecipazione nel quadro europeo: la cogestione, in DLRI, 1991, p. 1.

M. Pedrazzoli, Partecipazione, costituzione economica e art. 46 della Costituzione. Chiose e distinzioni sul declino di un'idea, in RIDL, 2005, I, p. 427.

M. Pedrazzoli, La partecipazione dei lavoratori: un tema di natura costituzionale e politica, in C. ZolI (a cura di), Lavoro e impresa: la partecipazione dei lavoratori e le sue forme nel diritto italiano e comparato, Torino, Giappichelli, 2015, p. 1.

J. Pélissier, G. Auzero, E. Dockès, Droit du travail, Paris, Dalloz, 2012.

C. Pelz, $\S 25$ Bußgeldvorschriften, in N. Kollmer, D.T. Klindt, Arbeitsschutzgesetz Kommentar, München, Verlag C.H. Beck, 2011, p. 597.

F. Pérez de los Cobos Orihuel, La ley de prevención de riesgos laborales como transposición de la directiva marco sobre seguridad y salud de los trabajadores en el lugar de trabajo, in Tribuna social, 1997, p. 7.

P. Perlingieri, Prassi, principio di legalità e scuole civilistiche, in ID., Scuole, tendenze e metodi. Problemi di diritto civile, Napoli, Edizioni Scientifiche Italiane, 1989, p. 215.

M. Persiani, Saggio sull'autonomia privata collettiva, Padova, Cedam, 1972.

A. Perulli, Il Testo Unico sul sistema di relazioni sindacali e di partecipazione della Electrolux-Zanussi, in LD, 1999, p. 41.

A. Perulli, Ascesa e declino della partecipazione alla Zanussi Electrolux, in DLM, 2005, p. 615.

A. Perulli, Intervento, in Rappresentanza collettiva dei lavoratori e diritti di partecipazione alla gestione delle imprese, Atti delle giornate di studio di diritto del lavoro, Lecce, 27-28 maggio 2005, Milano, Giuffrè, 2006, p. 304.

A. Perulli, La responsabilità sociale dell'impresa e i codici di condotta: 
modelli ed efficacia, in L. Montuschi, P. Tullini (a cura di), Lavoro e responsabilità sociale dell'impresa, Bologna, Zanichelli, 2006, p. 121.

E. Peskine, C. Wolmark, Droit du travail, Paris, Dalloz, 2016.

C. Piergallini, Il modello organizzativo alla verifica della prassi, in Le Società, 2011, p. 47.

C. Piergallini, Paradigmatica dell'autocontrollo penale (dalla funzione alla struttura del "modello organizzativo" ex d.lgs. 231/2001), Relazione all'incontro di studio del Consiglio Superiore della Magistratura sul tema «Le tipologie di colpa penale tra teoria e prassi», 28-30 marzo 2011, in www.csm.it.

C. Piergallini, Colpa e attività produttive: un laboratorio di diritto "cedevole”, in G. Casaroli, F. Giunta, R. Guerrini, A. Melchionda (a cura di), La tutela penale della sicurezza del lavoro, Pisa, Edizioni ETS, 2015, p. 379.

N. Pisani, Profili penalistici del testo unico sulla salute e sicurezza sui luoghi di lavoro, in Diritto penale e processo, 2008, n. 7, p. 827.

A. Pizzoferrato, L'autonomia collettiva nel nuovo diritto del lavoro, in DLRI, 2015, p. 411.

G. Proia, Consultazione e partecipazione dei lavoratori, in L. Montuschi (a cura di), Ambiente, salute e sicurezza. Per una gestione integrata dei rischi da lavoro, Torino, Giappichelli, 1997, p. 193.

G. Proia, La partecipazione dei lavoratori tra realtà e prospettive. Analisi della normativa interna, in DRI, 2010, p. 60.

D. Pulitanò, La responsabilità da reato degli enti: $i$ criteri d'imputazione, in RIDPP, 2002, I, p. 425.

G. QuADRI, Usi aziendali e autonomia negoziale. Regole consuetudinarie e modificazioni unilaterali nel rapporto di lavoro, Napoli, Edizioni Scientifiche Italiane, 2008.

M. RaвitTI, Modelli organizzativi e responsabilità degli enti: l'incerta figura dell'organismo di vigilanza, in Resp. amm. soc. enti, 2010, p. 27.

I. Regalia, Riflettendo ancora sulla partecipazione diretta, in L'impresa al plurale. Quaderni della partecipazione, 2000, p. 15.

I. Regalia, Per una riconfigurazione dell'azione sindacale, in Quaderni di rassegna sindacale, 2015, n. 1, p. 183.

S. Renga, Modello sindacale di tutela della salute nei luoghi di lavoro dal dopoguerra agli anni novanta, in LD, 1994, n. 4, p. 615.

M. Ricci, Ambiente di lavoro e responsabilità sociale d'impresa, in L. MoNTuschi, P. Tullini (a cura di), Lavoro e responsabilità sociale dell'impresa, Bologna, Zanichelli, 2006, p. 33. 
M. Ricci, Sicurezza sul lavoro: controllo e partecipazione sindacale tra iure condito $e$ de iure condendo, in Scritti in onore di Edoardo Ghera, II, Bari, Cacucci, 2008, p. 981.

M. Ricci, Gli organismi paritetici e il fondo di sostegno, in L. Zoppoli, P. Pascucci, G. NAtullo (a cura di), Le nuove regole per la salute e la sicurezza dei lavoratori, Milano, Ipsoa, 2010, p. 521.

R. Richardi (hrsg.), Betriebsverfassungsgesetz mit Wahlordnung, München, Verlag C.H. Beck, 2016.

S. Rodotà, Le fonti di integrazione del contratto, Milano, Giuffrè, 2004.

R. Romer, L'autonomia collettiva nella dottrina giuslavoristica: rileggendo Gaetano Vardaro, in DLRI, 2011, p. 181.

R. RomeI, Il contratto collettivo nel nuovo scenario di relazioni industriali, in L. Corazza, R. Romei (a cura di), Diritto del lavoro in trasformazione, Bologna, Il Mulino, 2014, p. 81.

J. Romeral Hernández, Consulta y participación de los trabajadores, in A.V. Sempere Navarro (dir.), M. Cardenal Carro, I. Alzaga Ruiz (coord.), Comentarios a la Ley de Prevención de Riesgos Laborales, Navarra, Aranzadi, 2010, p. 660.

M. Rusciano, A proposito del "Libro Bianco sul mercato del lavoro in Italia”, in www.unicz.it/lavoro/lavoro.htm, 2002.

M. Rusciano, Contrattazione e sindacato nel diritto del lavoro dopo la l. 28 giugno 2012, n. 92, in Studi in memoria di Mario Giovanni Garofalo, II, Bari, Cacucci, 2015, p. 867.

R. SAcco, Il contratto, in Trattato di diritto civile italiano, diretto da F. Vassalli, Torino, Utet, 1975.

R. SAcco, La conclusione dell'accordo, in E. Gabrielli (a cura di), I contratti in generale, Torino, Utet, 2006, p. 79.

C. Sachs-Durand, Occupational Health and Safety in France: A Good Formal Protection, but a Problematic Efficiency, in E. Ales (ed.), Health and Safety at Work. European and Comparative Perspective, Alphen aan den Rijn, Wolters Kluwer, 2013, p. 99.

R. SAlomone, Metodo comparato e misurazione del diritto del lavoro, in $\mathrm{F}$. BASENGHI, L.E. Golzio (a cura di), Regole, politiche e metodo. L'eredità di Marco Biagi nelle relazioni di lavoro di oggi, Torino, Giappichelli, 2013, p. 76.

C. Sánchez Trigueros, F.A. González Díaz (dir.), Libertad de empresa y poder de dirección del empresario en las relaciones laborales, Navarra, Aranzadi, 2011.

A.M. SAndulli, Manuale di diritto amministrativo, Napoli, Jovene, 1989. 
C. Santoriello, Qual è la posizione dei componenti dell'OdV rispetto agli infortuni sul lavoro e le malattie professionali?, in Resp. amm. soc. enti, 2015, n. 2, p. 71.

F. Santoro Passarelli, voce Autonomia collettiva, in Enc. dir., Milano, Giuffré, 1959, p. 369.

F. Santoro Passarelli, Nozioni di diritto del lavoro, Napoli, Jovene, 1961.

F. Santoro Passarelli, Il sindacato nell'impresa, in RDL, 1976, I, p. 5.

R. Scognamiglio, Dei contratti in generale. Artt. 1321-1352, in A. Scialoja, G. Branca (a cura di), Commentario del codice civile, Bologna-Roma, Zanichelli-Il Foro italiano, 1970.

L. Senden, Soft law in European Community Law, Oxford and Portland, Hart Publishing, 2004.

J.M. Servais, International Labour Law, Alphen aan den Rijn, Wolters Kluwer, 2011.

C. SmuRAglia, Il comportamento concludente nel rapporto di lavoro, Milano, Giuffrè, 1963.

C. Smuraglia, La sicurezza del lavoro e la sua tutela penale, Milano, Giuffrè, 1974.

C. Smuraglia, Sicurezza e igiene del lavoro. Quadro normativo. Esperienze attuative e prospettive, in RGL, 2001, I, p. 465.

C. Smuraglia, Sicurezza del lavoro e obblighi comunitari. I ritardi dell'Italia nell'adempimento e le vie per uscirne, in RIDL, 2002, p. 183.

J.L. SouRIoux, Rapport Français, in Le rôle de la pratique dans la formation du droit. Travaux de l'Association H. Capitant, Tome XXXIV, 1983, p. 85.

L. Spagnuolo Vigorita, Gli usi aziendali, Napoli, Morano, 1965.

L. Spagnuolo Vigorita, Usi aziendali e diritto del lavoro, in MGL, 1989, p. 684.

L. Spagnuolo Vigorita, voce Usi aziendali, in Enc. dir., Aggiornamento, Milano, Giuffré, 2001, p. 1118.

V. Speziale, La nuova legge sulla sicurezza del lavoro, in WP C.S.D.L.E. "Massimo D'Antona".IT-60/2007.

G. SuPPIEJ, Il diritto dei lavoratori alla salubrità dell'ambiente di lavoro, in RIDL, 1988, p. 442.

A. TAmpieri, Sicurezza sul lavoro e modelli di rappresentanza, Torino, Giappichelli, 1999.

F. TedeschinI, voce Omologazione, in Enc. dir., XXX, Milano, Giuffrè, 1980, p. 86.

B. Teyssí, Droit du travail. Relations collectives, Paris, Lexis Nexis, 2014. 
M. TiRaboschi, Il sistema di qualificazione delle imprese e dei lavoratori autonomi, in M. Tiraboschi, L. Fantini (a cura di), Il Testo Unico della salute e sicurezza sul lavoro dopo il correttivo (d.lgs. n. 106/2009), Milano, Giuffrè, 2009, p. 119.

M. Tiraboschi, Contrattazione collettiva: un'analisi empirica del periodo 2012-2014, in Contratti \& Contrattazione Collettiva, 2015, n. 4, p. 4.

M. Tiraboschi, Riforma della contrattazione: la proposta sindacale, in Contratti \& Contrattazione Collettiva, 2016, n. 2, p. 4.

$\mathrm{V}$. Torre, La valutazione del rischio e il ruolo delle fonti private, in D. CAstronuovo, F. Curi, S. Tordini Cagli, V. Torre, V. Valentini, Diritto penale della sicurezza sul lavoro, Bologna, Bononia University Press, 2016, p. 37.

P. Tosi, Informazione, consultazione e partecipazione dei lavoratori nelle imprese e nei gruppi di imprese della Cee, in Le ragioni del diritto. Scritti in onore di Luigi Mengoni, Milano, Giuffrè, 1995, p. 1305.

T. TReu, Cogestione e partecipazione, in DLRI, 1989, p. 597.

T. TREU, Le proposte parlamentari sulla partecipazione, in DRI, 2010, p. 93.

T. Treu, Flessibilità e tutele nella riforma del lavoro, in WP C.S.D.L.E. "Massimo D'Antona".IT-155/2012.

T. Treu, Introduzione Welfare aziendale, in WP C.S.D.L.E. "Massimo D'Antona”.IT-297/2016.

L. TRIA, Il difficile raggiungimento dell'obiettivo del benessere organizzativo nel lavoro, in http://www.europeanrights.eu.

A. Trojsi, La potestà legislativa dello Stato e delle Regioni, in L. Zoppoli, P. Pascucci, G. Natullo (a cura di), Le nuove regole per la salute e la sicurezza dei lavoratori, Milano, Ipsoa, 2010, p. 15.

G. Tudela Cambronero, Y. Valdeolivas García, La seguridad y la salud laboral en la negociación colectiva, Informes y Estudios, Relaciones Laborales, n. 88, Ministerio de Trabajo e Inmigración, Madrid, 2009.

P. TULLINI, Uso aziendale e principio volontaristico, in RIDL, 1991, II, p. 806.

P. Tuluini, Sicurezza sul lavoro e modello sociale europeo: un'ipotesi di sviluppo, in Scritti in onore di Edoardo Ghera, Bari, Cacucci, 2008, II, p. 1257.

P. TuluINI, Le competenze legislative in materia di sicurezza sul lavoro: $i$ nodi irrisolti, in Le Istituzioni del Federalismo, 2009, suppl. n. 1, p. 15.

P. Tuluini, Legge e legislazione lavoristica, in M. PeRsiani (a cura di), Le 
fonti del diritto del lavoro, Trattato di diritto del lavoro, diretto da M. Persiani e F. Carinci, Padova, Cedam, 2010, p. 451.

P. TulLinI, I sistemi di gestione della prevenzione e della sicurezza sul lavoro, in DLRI, 2010, p. 403.

P. TulLinI, Tutela civile e penale della sicurezza del lavoro: principi, categorie e regole probatorie a confronto, in RTDPC, 2011, p. 727.

P. TulLin, La responsabilità civile per esposizione lavorativa all'amianto: obbligo di sicurezza, limiti dell'esonero assicurativo e risarcibilità del danno, in RDSS, 2016, n. 1, p. 41.

F. VALdÉs DaL-RÉ, La participación de los trabajadores en la Ley de prevención de riesgos laborales, in Derecho y Salud, 1996, vol. IV, p. 71.

F. VALDÉs DAL-RÉ, Le relazioni industriali nel sistema spagnolo: una panoramica generale, in L. Fiorillo, A. Perulli (dir.), Le relazioni industriali, Torino, Giappichelli, 2014, vol. III, p. 295.

L. Valente, Consuetudini e usi nel rapporto di lavoro subordinato, Milano, Giuffrè, 2006.

B. Veneziani, Stato e autonomia collettiva. Diritto sindacale italiano e comparato, Bari, Cacucci, 1992.

P.-Y. VerKInDT, Le représentant syndical au comité d'hygiène, de sécurité et des conditions de travail, in Droit Social, 2009, n. 2, p. 181.

P.-Y. VerKInDt, Quand les conditions de travail s'invitent dans la sécurisation de l'emploi, in Droit Social, 2013, p. 730.

E. VILLANI, La responsabilità penale delle persone giuridiche per gli infortuni sul lavoro in Francia, in A.M. Stile, A. Fiorella, V. Mongillo (a cura di), Infortuni sul lavoro e doveri di adeguata organizzazione: dalla responsabilità penale individuale alla "colpa" dell'ente, Napoli, Jovene, 2014, p. 513.

A. Vimercati, B. Veneziani, La partecipazione in Europa tra Costituzione, legge e contrattazione collettiva, in L'impresa al plurale. Quaderni della partecipazione, 1999, n. 3-4, p. 279.

M. VINCIERI, Buone prassi e modelli di organizzazione e gestione nella tutela della sicurezza sul lavoro: distinzioni e possibili interferenze, in D. Fondaroli, C. Zoli (a cura di), Modelli organizzativi ai sensi del D.Lgs. n. 231/2001 e tutela della salute e della sicurezza nei luoghi di lavoro, Torino, Giappichelli, 2014, p. 72.

M. VINCIERI, Attività sindacale e benessere organizzativo dei lavoratori, in DLM, 2016, n. 3, p. 631.

A. VIscomi, Cresce la partecipazione con le norme sulla sicurezza, in $L I$, 1995, n. 11, p. 13. 
T. Vitarelli, Infortuni sul lavoro e responsabilità degli enti: un difficile equilibrio normativo, in RIDPP, 2009, p. 707.

D. WALTERS, Employee representation and health and safety: A strategy for improving health and safety performance in small enterprises?, in Employee Relations, 1998, vol. 20, p. 180.

D. Walters, T. Nichols, J. Connor, A.C. Tasiran, S. Cam, The role and effectiveness of safety representatives in influencing workplace health and safety, Health and safety executive, 2005, in www.hse.gov.uk.

D. Walters, R. Johnstone, K. Frick, M. Quinlan, G. Baril-Gingras, A. Thébaud-Mony, Regulating Workplace Risks. A Comparative Study of Inspection Regimes in Times of Changes, Cheltenham, Edward Elgar Publishing, 2011.

D. Walters, E. Wadsworth, The United Kingdom, in D. Walters, E. Wadsworth, M. Quinlan, Analysis of the determinants of workplace occupational safety and health practice in a selection of EU Member States, Cardiff Work Environment Research Centre, Cardiff University, 2012, p. 117.

Lord Wedderburn of Charlton, G. Giugni, E. Ghera, G.F. Mancini, U. Romagnoli, G. Minervini, B. Trentin, Democrazia politica e democrazia industriale. Cogestione, partecipazione e controllo in Italia e in Europa, Bari, De Donato, 1978.

M. Weiss, L'effettività del diritto del lavoro: alcune riflessioni sull'esperienza tedesca, in RIDL, 2006, I, p. 141.

C. Wolmark, Comité d'hygiène, de sécurité et des conditions de travail, in P. Zawieja, F. Guarnieri (sous la direction de), Dictionnaire des risques psychosociaux, Paris, Le Seuil, 2014, p. 114.

U. ZACHERT, Lezioni di diritto del lavoro tedesco, in Quaderni del Dipartimento di Scienze Giuridiche dell'Università degli Studi di Trento, 1995.

G. Zagrebelsky, Il diritto mite. Legge, diritti, giustizia, Torino, Einaudi, 1992.

C. Zous, La tutela delle posizioni «strumentali» del lavoratore. Dagli interessi legittimi all'uso delle clausole generali, Milano, Giuffrè, 1988.

C. ZoLI, Le clausole di procedimentalizzazione dei poteri imprenditoriali, in M. D'Antona (a cura di), Letture di diritto sindacale, Napoli, Jovene, 1990, p. 379.

C. ZoLI, Gli obblighi a trattare nel sistema dei rapporti collettivi, Padova, Cedam, 1992.

C. Zoul, Sicurezza del lavoro: contrattazione e partecipazione, in RGL, 2000, p. 613. 
C. Zoli, Sicurezza del lavoro e certezza del diritto, Intervento, in A. VAlLEBONa (a cura di), Colloqui Giuridici sul Lavoro, Milano, 2009, p. 54.

C. Zoli, Fidelizzazione del lavoratore, in Lessico giuslavoristico, ordinato da M. Pedrazzoli, vol. 2, Bologna, Bononia University Press, 2010, p. 37.

C. Zoli, Artt. 47-50, in ID. (a cura di), Principi comuni, in L. Montuschi (dir.), La nuova sicurezza sul lavoro, vol. I, Bologna, Zanichelli, 2011, p. 507.

C. Zoli, Art. 51, in ID. (a cura di), Principi comuni, in L. Montuschi (dir.), La nuova sicurezza sul lavoro, vol. I, Bologna, Zanichelli, 2011, p. 527.

C. Zoli, La partecipazione dei lavoratori alla gestione delle imprese nella recente progettazione legislativa, in ID. (a cura di), Lavoro e impresa: la partecipazione dei lavoratori e le sue forme nel diritto italiano e comparato, Torino, Giappichelli, 2015, p. 109.

L. Zoppoli, Rappresentanza collettiva dei lavoratori e diritti di partecipazione alla gestione delle imprese, in DLRI, 2005, p. 373.

L. Zoppoli, Modelli partecipativi e tecniche di regolazione dei rapporti di lavoro, in DRI, 2010, p. 19.

L. ZoppoLI, Il controllo collettivo sull'efficace attuazione del modello organizzativo per la sicurezza nei luoghi di lavoro, in D. Fondaroli, C. ZoLI (a cura di), Modelli organizzativi ai sensi del D.Lgs. n. 231/2001e tutela della salute e della sicurezza nei luoghi di lavoro, Torino, Giappichelli, 2014, p. 12.

L. Zoppoli, A. Zoppoli, M. Delfino (a cura di), Una nuova Costituzione per il sistema di relazioni sindacali?, Napoli, Editoriale Scientifica, 2014. 


\section{SOMMARIO}

\section{CAPITOLO I \\ LE BUONE PRASSI IN MATERIA DI SALUTE E SICUREZZA NEI LUOGHI DI LAVORO}

1. Le buone prassi nel d.lgs. n. 81/2008

2. La nozione legislativa e il raccordo con l'art. 2087 c.c. 19

3. Il ruolo degli organismi paritetici 25

4. L'atto di validazione 28

5. Contenuto e diffusione delle good practices a livello interno e dell'Unione europea $\quad 34$

6. Inquadramento e qualificazione giuridica 41

6.1. Le buone prassi quali atti unilaterali integrativi del contratto di lavoro

7. Volontarietà e necessità

7.1. Quale incidenza sui profili di responsabilità? Alcune ipotesi possibili

8. Spunti per una comparazione

8.1. L'esperienza spagnola, francese e tedesca

8.2. Gli Approved Codes of Practice e le guidances nell'ordinamento britannico

\section{Capitolo II \\ LA FORMAZIONE DI REGOLE IN AZIENDA TRA CONTRATTAZIONE E PARTECIPAZIONE}

1. Il ruolo della contrattazione e della partecipazione nella formazione di regole

2. La promozione della salute nel lavoro attraverso la contrattazione collettiva aziendale

3. Natura ed efficacia delle misure prevenzionistiche contrattualizzate

4. Contrattazione collettiva e prevenzione dei rischi nell'ordinamento spagnolo: un esempio affine

5. La «partecipazione equilibrata» nel contesto italiano 


\section{CAPITOLO III \\ IL RILIEVO DELLA DIMENSIONE PARTECIPATIVA \\ IN ALTRI ORDINAMENTI NAZIONALI}

1. La diversa interpretazione del meccanismo partecipativo ai fini della tutela della sicurezza nei luoghi di lavoro

2. Il dovere di consultazione dei rappresentanti dei lavoratori nel Regno Unito e l'ipotesi del Safety Committee

3. Il Comité d'hygiène, de sécurité et des conditions de travail

4. Il Comité de Seguridad y Salud

5. Gli accordi di codeterminazione nella tutela della sicurezza sul lavoro (Betriebsvereinbarungen)

5.1. Gli accordi aziendali volontari (freiwillige Betriebsvereinbarungen)

6. Brevi riflessioni conclusive

\section{Capitolo IV \\ I MODELLI DI ORGANIZZAZIONE E GESTIONE DELLA SICUREZZA}

1 La valorizzazione dell'adozione ed efficace attuazione dei modelli di organizzazione e gestione ex art. 30 d.lgs. n. 81/2008

1.2. L'asseverazione da parte degli organismi paritetici

2. Tra volontarietà, onere e obbligo

3. Modelli organizzativi, buone prassi e codici etici: analogie, differenze e commistioni

4. Cenni sull'efficacia esimente della responsabilità amministrativa delle persone giuridiche in altri contesti nazionali 


\section{P U B B L I C A Z I O N I D E L S E M I N A R I O G I U R I D I C O DELLA UNIVERSITÀ DI BOLOGNA}

1. CoLI U., Collegia et sodalitates, 1913.

2. Donatelli I., La "consortia" di Avesa, 1914.

3. Valenza P., Il diritto di usufrutto nelle leggi sulle tasse del registro, 1915.

4. Zingali G., La statistica della criminalità, 1916.

5. Tumedei C., La separazione dei beni ereditari, 1917.

6. Albertoni A., L“Apokeryxis", 1923.

7. SALVI F., La cessione dei beni ai creditori, 1947.

8. Milani F., Distinzioni delle servitù prediali, 1948.

9. FAssò G., I "quattro autori" del Vico, 1949.

10. FERRI L., La trascrizione degli acquisti "mortis causa" e problemi connessi, 1951.

11. Rossi G., La "Summa arboris actionum" di Ponzio da Ylerda, 1951.

12. Poggeschi R., Le associazioni e gli altri gruppi con autonomia patrimoniale nel processo, 1951.

13. Matteucci N., Antonio Gramsci e la filosofia della prassi, 1951.

14. Forchielli P., I contratti reali, 1952.

15. SAlvi F., Il possesso di stato familiare, 1952.

16. FAssò G., La storia come esperienza giuridica, 1953.

17. Palazzini Finetti L., Storia della ricerca delle interpolazioni nel Corpus iuris giustinianeo, 1953.

18. Rossi G., Consilium sapientis iudiciale, 1958.

19. Mancini G.F., La responsabilità contrattuale del prestatore di lavoro, 1957.

20. FerRi L., L'autonomia privata, 1959.

21. Torelli P., Scritti di storia del diritto italiano, 1959.

22. Santini G., I Comuni di Valle del medioevo. La Costituzione federale del "Frignano", 1960.

23. Gianniti F., I reati della stessa indole, 1959.

24. GHezzi G., La prestazione di lavoro nella comunità familiare, 1960.

25. Nardi E., Case "infestate da spiriti" e diritto romano e moderno, 1960.

26. FERRI L., Rinunzia e rifiuto nel diritto privato, 1960.

27. GHezzi G., La responsabilità contrattuale delle associazioni sindacali, 1963.

28. Bonsignori A., Espropriazione della quota di società a responsabilità limitata, 1961.

29. Redenti E., Scritti e discorsi giuridici di un mezzo secolo, vol. I, Intorno al diritto processuale, 1962.

30. Redenti E., Scritti e discorsi giuridici di un mezzo secolo, vol. II, Intorno al diritto sostanziale, 1962.

31. Gualandi A., Spese e danni nel processo civile, 1962.

32. Bonsignori A., Assegnazione forzata e distribuzione del ricavato, 1960.

33. Mancini G.F., Il recesso unilaterale e i rapporti di lavoro, vol. I, Individuazione della fattispecie. Il recesso ordinario, 1962.

34. NARDI E., Rabelais e il diritto romano, 1962.

35. Romagnoli U., Il contratto collettivo di impresa, 1963.

36. Santini G., I "comuni di pieve" nel medioevo italiano, 1964.

37. Rudan M., Il contratto di tirocinio, 1966.

38. Bonini R., I "libri de cognitionibus" di Callistrato. Ricerche sull'elaborazione giurisprudenziale della "cognitio extra ordinem", 1964.

39. Colliva P., Ricerche sul principio di legalità nell'amministrazione del Regno di Sicilia al tempo di Federico II, 1964.

40. Mengozzi P., L'agenzia di approvvigionamento dell'Euratom, 1964.

41. Scritti minori di Antonio Cicu, tomi I e II, Scritti di teoria generale del diritto - Diritto di famiglia, 1965.

42. Scritti minori di Antonio Cicu, Successioni e donazioni. Studi vari, 1965.

43. SACChI Morsiani G., Il potere amministrativo delle Comunità europee e le posizioni giuridiche dei privati, I, 1965.

44. GHEzzI G., La mora del creditore nel rapporto di lavoro, 1965.

45. Roversi Monaco F.A., Enti di gestione. Struttura, funzioni, limiti, 1967.

46. Gianniti F., L'oggetto materiale del reato, 1966. 
47. Mengozzi P., L'efficacia in Italia di atti stranieri di potestà pubblica su beni privati, 1967.

48. Romagnoli U., La prestazione di lavoro nel contratto di società, 1967.

49. Montuschi L., I limiti legali nella conclusione del contratto di lavoro, 1967.

50. Ranieri S., Scritti e discorsi vari, vol. I, Scritti di diritto penale, 1968.

51. Ranieri S., Scritti e discorsi vari, vol. II, Scritti di procedura penale, 1968.

52. BoNINI R., Ricerche di diritto giustinianeo, 1968.

53. SANTINI G., Ricerche sulle "Exceptiones legum romanorum", 1969.

54. Lo Castro G., La qualificazione giuridica delle deliberazioni conciliari delle fonti del diritto canonico, 1970.

55. Sacchi Morsiani G., Il potere amministrativo delle Comunità europee e le posizioni giuridiche dei privati, II, 1970.

56. Roversi Monaco F.A., La delegazione amministrativa nel quadro dell'ordinamento regionale, 1970.

57. Gianniti F., Studi sulla corruzione del pubblico ufficiale, 1970.

58. De Vergottini G., Indirizzo politico della difesa e sistema costituzionale, 1971.

59. Mengozzi P., Il regime giuridico internazionale del fondo marino, 1971.

60. CaRinci F., Il conflitto collettivo nella giurisprudenza costituzionale, 1971.

61. Osti G., Scritti giuridici, voll. I e II, 1973.

62. Zuelli F., Servizi pubblici e attività imprenditoriale, 1973.

63. Pergolesi F., Sistema delle fonti normative, 1973.

64. Montuschi L., Potere disciplinare e rapporto di lavoro, 1973.

65. Pattaro E., Il pensiero giuridico di L.A. Muratori tra metodologia e politica, 1974.

66. PINI G., Arbitrato e lavori pubblici, 1974.

67. CARPI F., L'efficacia "ultra partes" della sentenza civile, 1974.

68. De Vergottini G., Lo "Shadow cabinet", 1973.

69. PaOLuCCI L.F., La mutualità nelle cooperative, 1974.

70. De Gennaro A., Crocianesimo e cultura giuridica italiana, 1974.

71. Stortoni L., L'abuso di potere nel diritto penale, 1978.

72. Gianniti F., Prospettive criminologiche e processo penale, 1977.

73. Bonvicini D., Le "joint ventures": tecnica giuridica e prassi societaria, 1977.

74. De Vergottini G., Scritti di storia del diritto italiano, voll. I, II, III, 1977.

75. Lambertini R., I caratteri della Novella 118 di Giustiniano, 1977.

76. DALLA D., L'incapacità sessuale in diritto romano, 1978.

77. Di Pietro A., Lineamenti di una teoria giuridica dell'imposta sull'incremento di valore degli immobili, 1978.

78. Mazzacuva N., La tutela penale del segreto industriale, 1979.

79. Romanelli G., Profilo del noleggio, 1979.

80. Borghesi D., Il contenzioso in materia di eleggibilità, 1979.

81. Dalla Torre G., L'attività assistenziale della Chiesa nell'ordinamento italiano, 1979.

82. CARPI F., La provvisoria esecutorietà della sentenza, 1979.

83. Alleva P., Il campo di applicazione dello statuto dei lavoratori, 1980.

84. Puliatti S., Ricerche sulla legislazione "regionale" di Giustiniano, 1980.

85. Fassò G., Scritti di filosofia del diritto, voll. I, II, III, 1982.

86. SGUbBi F., Uno studio sulla tutela penale del patrimonio, 1980.

87. Lambertini R., Plagium, 1980.

88. DaLLa D., Senatus consultum Silanianum, 1980.

89. VANDELLI L., L'ordinamento regionale spagnolo, 1980.

90. NARDI E., L'otre dei parricidi e le bestie incluse, 1980.

91. Pellicanò A., Causa del contratto e circolazione dei beni, 1981.

92. Giardini D., Politica e amministrazione nello Stato fondato sul decentramento, 1981.

93. Bortolotti D., Potere pubblico e ambiente, 1981.

94. Roffi R., Contributo per una teoria delle presunzioni nel diritto amministrativo, 1982.

95. Alessi R., Scritti minori, 1981.

96. Bassanelli Sommariva G., L'imperatore unico creatore ed interprete delle leggi e l'autonomia del giudice nel diritto giustinianeo, 1983.

97. Zалотті A., Cultura giuridica del Seicento e jus publicum ecclesiasticum nell'opera del cardinal Giovanni Battista De Luca, 1983.

98. Illuminati G., La disciplina processuale delle intercettazioni, 1983.

99. Toniatti R., Costituzione e direzione della politica estera negli Stati Uniti d'America, 1983.

100. NARDI E., Squilibrio e deficienza mentale in diritto romano, 1983. 
101. Dalla D., Praemium emancipationis, 1983.

102. Mazzacuva N., Il disvalore di evento nell'illecito penale - L'illecito commissivo doloso e colposo, 1983.

103. Studi in onore di Tito Carnacini. I. Studi di diritto costituzionale, civile, del lavoro, commerciale, 1983.

104. CAIA G., Stato e autonomie locali nella gestione dell'energia, 1984.

105. Baratti G., Contributo allo studio della sanzione amministrativa, 1984.

106. Bontolotті D., Attività preparatoria e funzione amministrativa, 1984.

107. Puliatti S., Ricerche sulle novelle di Giustino II. La legislazione imperiale da Giustiniano I a Giustino II, 1984.

108. LAMBERTINi R., La problematica della commorienza nell'elaborazione giuridica romana, 1984.

109. ZuelLI F., Le collegialità amministrative, 1985.

110. Pedrazzoli M., Democrazia industriale e subordinazione, 1985.

111. Zanotтi M., Profili dogmatici dell'illecito plurisoggettivo, 1985.

112. Ruffolo U., Interessi collettivi o diffusi e tutela del consumatore, I, 1985.

113. Biagi M., Sindacato democrazia e diritto, 1986.

114. Insolera G., Problemi di struttura del concorso di persone nel reato, 1986.

115. Malagù L., Esecuzione forzata e diritto di famiglia, 1986.

116. RiCCI G.F., La connessione nel processo esecutivo, 1986.

117. ZanotTi A., Il concordato austriaco del 1855, 1986.

118. Selmini R., Profili di uno studio storico sull'infanticidio, 1987.

119. Dalla D., "Ubi venus mutatur", 1987.

120. Zunarelli S., La nozione di vettore, 1987.

121. Zoli C., La tutela delle posizioni "strumentali" del lavoratore, 1988.

122. Cavina M., Dottrine giuridiche e strutture sociali padane nella prima età moderna, 1988.

123. Califano L., Innovazione e conformità nel sistema regionale spagnolo, 1988.

124. Sarti N., Gli statuti della società dei notai di Bologna dell'anno 1336 (contributo allo studio di una corporazione cittadina), 1988.

125. SCARPONI S., Riduzione e gestione flessibile del tempo di lavoro, 1988.

126. Bernardini M., Contenuto della proprietà edilizia, 1988.

127. La Torre M., La "lotta contro il diritto soggettivo". Karl Larenz - la dottrina giuridica nazionalsocialista, 1988.

128. Garcia De Enterria J., Le obbligazioni convertibili in azioni, 1989.

129. Biagi Guerini R., Famiglia e Costituzione, 1989.

130. CaIa G., Arbitrati e modelli arbitrali nel diritto amministrativo, 1989.

131. Magagni M., La prestazione caratteristica nella Convenzione di Roma del 19 giugno 1980, 1989.

132. Petroni L., La disciplina pubblicistica dell'innovazione tecnologica in Francia, 1990.

133. Zanotti A., Le manipolazioni genetiche e il diritto della Chiesa, 1990.

134. SARTOR G., Le applicazioni giuridiche dell'intelligenza artificiale, 1990.

135. Rossi L.S., Il "buon funzionamento del mercato comune". Delimitazione dei poteri fra CEE e Stati membri, 1990.

136. Luchetti G., La legittimazione dei figli naturali nelle fonti tardo imperiali e giustinianee, 1990.

137. Sarti N., Un giurista tra Azzone e Accursio, 1990.

138. Gustapane A., La tutela globale dell'ambiente, 1991.

139. Bottari C., Principi costituzionali e assistenza sanitaria, 1991.

140. Donini M., Illecito e colpevolezza nell'imputazione del reato, 1991.

141. Perulli A., Il potere direttivo dell'imprenditore, 1992.

142. VANDELLI L. (a cura di), Le forme associative tra enti territoriali, 1992.

143. GASPARrI P., Institutiones iuris publici, 1992.

144. CAPuZzo E., Dal nesso asburgico alla sovranità italiana, 1992.

145. Biavati P., Accertamento dei fatti e tecniche probatorie nel processo comunitario, 1992.

146. Ferrari F., Atipicità dell'illecito civile. Una comparazione, 1992.

147. Gustapane A., Sartor G., Verardi C.M., Valutazione di impatto ambientale. Profili normativi e metodologie informatiche, 1992.

148. Orlandi R., Atti e informazioni della autorità amministrativa nel processo penale. Contributo allo studio delle prove extracostituite, 1992.

149. CaRPani G., Le aziende degli enti locali. Vigilanza e controlli, 1992. 
150. Musso A., Concorrenza ed integrazione nei contratti di subfornitura industriale, 1993.

151. Donini M., Il delitto contravvenzionale. "Culpa iuris" e oggetto del dolo nei reati a condotta neutra, 1993.

152. Califano Placci L., Le commissioni parlamentari bicamerali nella crisi del bicameralismo italiano, 1993.

153. ForNaSARI G., Il concetto di economia pubblica nel diritto penale. Spunti esegetici e prospettive di riforma, 1994.

154. ManZini P., L'esclusione della concorrenza nel diritto antitrust italiano, 1994.

155. Ттмотео M., Le successioni nel diritto cinese. Evoluzione storica ed assetto attuale, 1994.

156. Sesta M. (a cura di), Per i cinquant'anni del codice civile, 1994.

157. Tullini P., Contributo alla teoria del licenziamento per giusta causa, 1994.

158. Rescigno F., Disfunzioni e prospettive di riforma del bicameralismo italiano: la camera delle regioni, 1995.

159. Lugaresi N., Le acque pubbliche. Profili dominicali, di tutela, di gestione, 1995.

160. SARTI N., Maximum dirimendarum causarum remedium. Il giuramento di calunnia nella dottrina civilistica dei secoli XI-XIII, 1995.

161. Colliva P., Scritti minori, 1996.

162. Dugato M., Atipicità e funzionalizzazione nell'attività amministrativa per contratti, 1996.

163. Gardini G., La comunicazione degli atti amministrativi. Uno studio alla luce della legge 7 agosto 1990, n. 241, 1996.

164. Manzini P., I costi ambientali nel diritto internazionale, 1996.

165. Мıттіса M.P., Il divenire dell'ordine. L'interazione normativa nella società omerica, 1996.

166. Lucheтti G., La legislazione imperiale nelle Istituzioni di Giustiniano, 1996.

167. La Torre M., Disavventure del diritto soggettivo. Una vicenda teorica, 1996.

168. Самоn A., Le intercettazioni nel processo penale, 1996.

169. Mancini S., Minoranze autoctone e Stato. Tra composizione dei conflitti e secessione, 1996.

170. Zanobetti Pagnetti A., La non comparizione davanti alla Corte internazionale di giustizia, 1996.

171. Bricola F., Scritti di diritto penale. Vol. I, Dottrine generali, Teoria del reato e sistema sanzionatorio. Vol. II, Parte speciale e legislazione complementare, Diritto penale dell'economia, 1997.

172. Graziosi A., La sentenza di divorzio, 1997.

173. Mantovani M., Il principio di affidamento nella teoria del reato colposo, 1997.

174. Biavati P., Giurisdizione civile, territorio e ordinamento aperto, 1997.

175. Ross G. (1916-1986), Studi e testi di storia giuridica medievale, a cura di Giovanni Gualandi e Nicoletta Sarti, 1997.

176. Pellegrini S., La litigiosità in Italia. Un'analisi sociologico-giuridica, 1997.

177. Boni G., La rilevanza del diritto dello Stato nell'ordinamento canonico. In particolare la canonizatio legum civilium, 1998.

178. Scritti in onore di Giuseppe Federico Mancini. Vol. I, Diritto del lavoro, 1998.

179. Scritti in onore di Giuseppe Federico Mancini. Vol. II, Diritto dell'Unione europea, 1998

180. Rossi A., Il GEIE nell'ordinamento italiano. Criteri di integrazione della disciplina, 1998.

181. Bongiovanni G., Reine Rechtslehre e dottrina giuridica dello Stato. H. Kelsen e la Costituzione austriaca del 1920, 1998.

182. CaPuto G., Scritti minori, 1998.

183. Garrido J.M., Preferenza e proporzionalità nella tutela del credito, 1998.

184. Bellodi Ansaloni A., Ricerche sulla contumacia nelle cognitiones extra ordinem, I, 1998.

185. Franciosi E., Riforme istituzionali e funzioni giurisdizionali nelle Novelle di Giustiniano. Studi su nov. 13 e nov. 80, 1998.

186. Cattabriga C., La Corte di giustizia e il processo decisionale politico comunitario, 1998.

187. Mancini L., Immigrazione musulmana e cultura giuridica. Osservazioni empiriche su due comunità di egiziani, 1998.

188. GustaPane A., L'autonomia e l'indipendenza della magistratura ordinaria nel sistema costituzionale italiano. dagli albori dello Statuto Albertino al crepuscolo della bicamerale, premessa di Giuseppe De Vergottini, 1999.

189. RICCI G.F., Le prove atipiche, 1999.

190. Canestrari S., Dolo eventuale e colpa cosciente. Ai confini tra dolo e colpa nella struttura delle tipologie delittuose, 1999.

191. Fassò G., La legge della ragione. Ristampa, a cura di Carla Faralli, Enrico Pattaro, Giampaolo Zucchini, 1999. 
192. FAssò G., La democrazia in Grecia. Ristampa, a cura di Carla Faralli, Enrico Pattaro, Giampaolo Zucchini, 1999.

193. Scarciglia R., La motivazione dell'atto amministrativo. Profili ricostruttivi e analisi comparatistica, 1999.

194. Briguglio F., "Fideiussoribus succurri solet", 1999.

195. Maltoni A., Tutela dei consumatori e libera circolazione delle merci nella giurisprudenza della Corte di giustizia, profili costituzionali, prefazione di Augusto Barbera, 1999.

196. Fondaroli D., Illecito penale e riparazione del danno, 1999.

197. Rossi L.S., Le convenzioni fra gli Stati membri dell'Unione europea, 2000.

198. Gragnoli E., Profili dell'interpretazione dei contratti collettivi, 2000.

199. Boni G., La rilevanza del diritto secolare nella disciplina del matrimonio canonico, 2000.

200. Lugaresi N., Internet, privacy e pubblici poteri negli Stati Uniti, 2000.

201. Lalatta Costerbosa M., Ragione e tradizione. Il pensiero giuridico ed etico-politico di Wilehlmvon Humboldt, 2000.

202. Semeraro P., I delitti di millantato credito e traffico di influenza, 2000.

203. VERZA A., La neutralità impossibile. Uno studio sulle teorie liberali contemporanee, 2000.

204. Lolli A., L'atto amministrativo nell'ordinamento democratico. Studio sulla qualificazione giuridica, 2000 .

205. BusetTo M.L., Giudice penale e sentenza dichiarativa di fallimento, 2000.

206. Campanella P., Rappresentatività sindacale: fattispecie ed effetti, 2000.

207. BRICOLA F., Scritti di diritto penale. Opere monografiche, 2000.

208. LASSANDARI A., Il contratto collettivo aziendale e decentrato, 2001.

209. Bianco A., Il finanziamento della politica in Italia, 2001.

210. Raffi A., Sciopero nei servizi pubblici essenziali. Orientamenti della Commissione di garanzia, 2001.

211. Piergigli V., Lingue minoritarie e identità culturali, 2001.

212. Cafaro S., Unione monetaria e coordinamento delle politiche economiche. Il difficile equilibrio tra modelli antagonisti di integrazione europea, 2001.

213. Morrone A., Il custode della ragionevolezza, 2001.

214. Masutti A., La liberalizzazione dei trasporti in Europa. Il caso del trasporto postale, 2002.

215. Zanotti A., Orlando F., L'itinerario canonistico di Giuseppe Caputo, 2002.

216. LupoI M.A., Conflitti transnazionali di giurisdizioni. Vol. I, Policies, metodi, criteri di collegamento. Vol. II, Parallel proceedings, 2002.

217. Lolli A., I limiti soggettivi del giudicato amministrativo. Stabilità del giudicato e difesa del terzo nel processo amministrativo, 2002.

218. Curi F., Tertium datur. Dal Common Law al Civil Law per una scomposizione tripartita dell'elemento soggettivo del reato, 2003.

219. Cottignola G., Studi sul pilotaggio marittimo, 2003.

220. Gardini G., L'imparzialità amministrativa tra indirizzo e gestione. Organizzazione e ruolo della dirigenza pubblica nell'amministrazione contemporanea, 2003.

221. Cevenini C., Virtual enterprises. Legal issues of the on-line collaboration between undertakings, 2003.

222. Monducci J., Diritto della persona e trattamento dei dati particolari, 2003.

223. Villecco Bettelli A., L'efficacia delle prove informatiche, 2004.

224. Zucconi Galdi Fonseca E., La convenzione arbitrale rituale rispetto ai terzi, 2004.

225. BRight R., Norme e conoscenza: dal testo giuridico al metadato, 2004.

226. Luchetti G., Nuove ricerche sulle istituzioni di Giustiniano, 2004.

227. Studi in memoria di Angelo Bonsignori, voll. I, II, 2004.

228. Piperata G., Tipicità e autonomia nei servizi pubblici locali, 2005.

229. Canestrari S., Foffani L. (a cura di), Il diritto penale nella prospettiva europea. Quali politiche criminali per l'Europa? Atti del Convegno organizzato dall'Associazione Franco Bricola (Bologna, 28 febbraio-2 marzo 2002), 2005.

230. Мемmo D., Miconi S. (a cura di), Broadcasting regulation: market entry and licensing. Regolamentazione dell'attività radiotelevisiva: accesso al mercato e sistema di licenze. Global Classroom Seminar, 2006.

230. Bis Briguglio F., Studi sul procurator, 2007.

231. Querzola L., La tutela anticipatoria fra procedimento cautelare e giudizio di merito, 2006.

232. TARozZI S., Ricerche in tema di registrazione e certificazione del documento nel periodo postclassico, 2006.

233. Bотті F., L'eutanasia in Svizzera, 2007. 
234. Fondaroli D., Le ipotesi speciali di confisca nel sistema penale, 2007.

235. Alagna R., Tipicità e riformulazione del reato, 2007.

236. Giovannini M., Amministrazioni pubbliche e risoluzione alternativa delle controversie, 2007.

237. Montalti M., Orientamento sessuale e costituzione decostruita. Storia comparata di un diritto fondamentale, 2007.

238. Tordini Cagli S., Principio di autodeterminazione e consenso dell'avente diritto, 2008.

239. Legnani Annichini A., La mercanzia di Bologna. Gli statuti del 1436 e le riformagioni quattrocentesche, 2008.

240. LoLLI A., L'amministrazione attraverso strumenti economici, 2008.

241. VACCARELla M., Titolarità e funzione nel regime dei beni civici, 2008.

242. Tubertini C., Pubblica amministrazione e garanzia dei livelli essenziali delle prestazioni, 2008.

243. Fioriglio G., Il diritto alla privacy. Nuove frontiere nell'era di Internet, 2008.

244. Вотті F., Manipolazioni del corpo e mutilazioni genitali femminili, 2009.

245. Nisco A., Controlli sul mercato finanziario e responsabilità penale. Posizioni di garanzia e tutela del risparmio, 2009.

246. Zanobetti Pagnetti A., Il rapporto internazionale di lavoro marittimo, 2008.

247. Matтioli F., Ricerche sulla formazione della categoria dei cosiddetti quasi delitti, 2010.

248. Bertaccini D., La politica di polizia, 2009.

249. Astrologo A., Le cause di non punibilità. Un percorso tra nuovi orientamenti interpretativi e perenni incertezze dogmatiche, 2009.

250. Di MARIA S., La cancelleria imperiale e $i$ giuristi classici: "Reverentia antiquitatis" e nuove prospettive nella legislazione giustinianea del codice, 2010.

251. Valentini E., La domanda cautelare nel sistema delle cautele personali, 2010.

252. Querzola L., Il processo minorile in dimensione europea, 2010.

253. Bologna C., Stato federale e "national interest". Le istanze unitarie nell'esperienza statunitense, 2010.

254. Rasia C., Tutela giudiziale europea e arbitrato, 2010.

255. Zucconi Galli Fonseca E., Pregiudizialità e rinvio (Contributo allo studio dei limiti soggettivi dell'accertamento), 2011.

256. Bellodi Ansaloni A., Ad eruendam veritatem. Profili metodologici e processuali della quaestio per tormenta, 2011.

257. Pontoriero I., Il prestito marittimo in diritto romano, 2011.

258. Giustizia senza confini. Studi offerti a Federico Carpi, 2012.

259. Gustapane A., Il ruolo del pubblico ministero nella Costituzione italiana, 2012.

260. Cainiello M., Premesse per una teoria del pregiudizio effettivo nelle invalidità processuali penali, 2012.

261. BRiguglio F., Il Codice Veronese in trasparenza. Genesi e formazione del testo delle Istituzioni di Gaio, 2012.

262. VALENTINi E., La domanda cautelare nel sistema delle cautele personali, Nuova edizione, 2012.

263. Tassinari D., Nemo tenetur se detegere. La libertà dalle autoincriminazioni nella struttura del reato, 2012.

264. Martelloni F., Lavoro coordinato e subordinazione. L'interferenza delle collaborazioni a progetto, 2012.

265. Roversi-Monaco F. (a cura di), Università e riforme. L'organizzazione delle Università degli Studi ed il personale accademico nella legge 30 dicembre 2010, n. 240, 2013.

266. Torre V., La privatizzazione delle fonti di diritto penale, 2013.

267. Raffotтa E.C., Il governo multilivello dell'economia. Studio sulle trasformazioni dello Stato costituzionale in Europa, 2013.

268. Caruso C., La libertà di espressione in azione. Contributo a una teoria costituzionale del discorso pubblico, 2013.

269. Pedrini F., Le "clausole generali". Profili teorici e aspetti costituzionali, 2013.

270. Curi F., Profili penali dello stress lavoro-correlato. L'homo faber nelle organizzazioni complesse, 2013.

271. CASALE D., L'idoneità psicofisica del lavoratore pubblico, 2013.

272. Nicodemo S., Le istituzioni della conoscenza nel sistema scolastico, 2013.

273. Legnani Annichini A., "Proxeneta est in tractando». La professione ingrata del mediatore di commercio (secc. XII-XVI), 2013.

274. Monducc J., Il dato genetico tra autodeterminazione informativa e discriminazione genotipica, 2013.

275. Mantovani M., Contributo ad uno studio sul disvalore di azione nel sistema penale vigente, 2014.

276. DE Donno M., Consensualità e interesse pubblico nel governo del territorio, 2015. 
277. Pacilli M., L'abuso dell'appello, 2015.

278. PIšTan Č., Tra democrazia e autoritarismo. Esperienze di giustizia costituzionale nell'Europa centro-orientale e nell'area post-sovietica, 2015.

279. Bellodi Ansaloni A., L'arte dell'avvocato, actor veritatis. Studi di retorica e deontologia forense, 2016.

280. Нохна D., La giustizia criminale napoleonica. A Bologna fra prassi e insegnamento universitario, 2016.

281. Querzola L., L'efficacia dell'attività processuale in un diverso giudizio, 2016.

282. Pieri B., Usurai, ebrei e poteri della Chiesa nei consilia di Paolo da Castro, 2016.

283. Rasia C., La crisi della motivazione nel processo civile, 2016.

284. Drigo C., Le Corti costituzionali tra politica e giurisdizione, 2016.

285. Polacchini F., Doveri costituzionali e principio di solidarietà, 2016.

286. Calcagnile M., Inconferibilità amministrativa e conflitti di interesse nella disciplina dell'accesso alle cariche pubbliche, 2017.

287. VILLA E., La responsabilità solidale come tecnica di tutela del lavoratore, 2017.

288. VINGIERI M., L'integrazione dell'obbligo di sicurezza, 2017. 


Finito di stampare nel mese di novembre 2017 per i tipi di Bononia University Press 